\title{
TABLES OF NEUTRON CROSS SECTIONS
}
by
K. Way
G. Haines

This document is

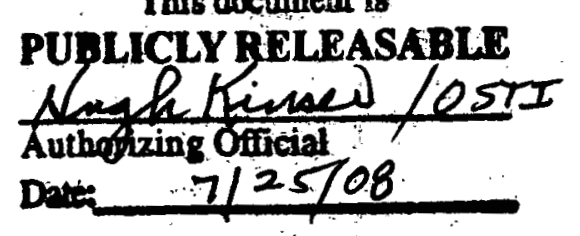

Oak Ridge National Laboratory

Date of Manuscript:

Date Declassified:

Supplement Declassified:
October 28,1947 July 22, 1948 August . 4, 1948

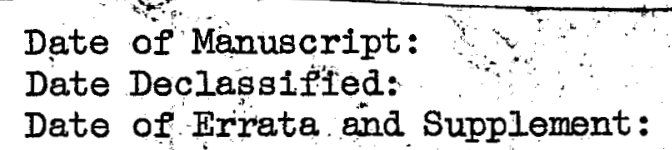

a.

\section{DECLASSIFIED}




\section{DISCLAIMER}

This report was prepared as an account of work sponsored by an agency of the United States Government. Neither the United States Government nor any agency Thereof, nor any of their employees, makes any warranty, express or implied, or assumes any legal liability or responsibility for the accuracy, completeness, or usefulness of any information, apparatus, product, or process disclosed, or represents that its use would not infringe privately owned rights. Reference herein to any specific commercial product, process, or service by trade name, trademark, manufacturer, or otherwise does not necessarily constitute or imply its endorsement, recommendation, or favoring by the United States Government or any agency thereof. The views and opinions of authors expressed herein do not necessarily state or reflect those of the United States Government or any agency thereof. 


\section{DISCLAIMER}

Portions of this document may be illegible in electronic image products. Images are produced from the best available original document. 


\section{TABLE OF CONTENTS}

Explanatory Remarks $\ldots . \ldots \ldots \ldots \ldots \ldots \ldots \ldots \ldots \ldots \ldots \ldots \ldots \ldots$

Purpose and Acknowledgements

Criteria for Omitting Measurements

Material of Tables

Abbreviations and Values of Neutron Energies

Adopted for Various Neutron Sources ................... 7

Tables of Neutron Cross Sections for All

Elements from Hydrogen through Bismuth ...................... 10

References Cited in Tables .......................... 189

References to Transport, Inelastic and Differential

Scattering Cross Sections ......................... 21

Errata and Supplement ................................ 215

$\Leftrightarrow$

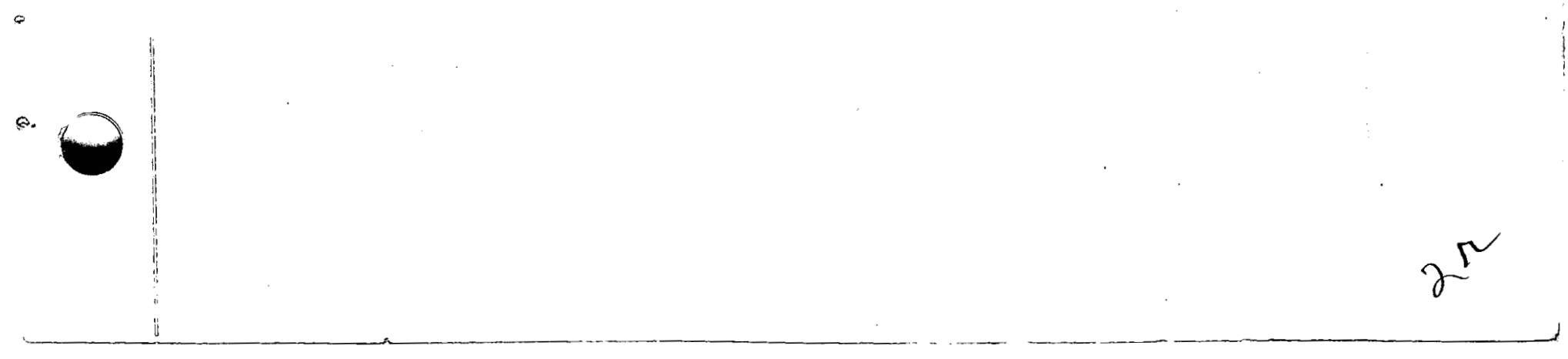




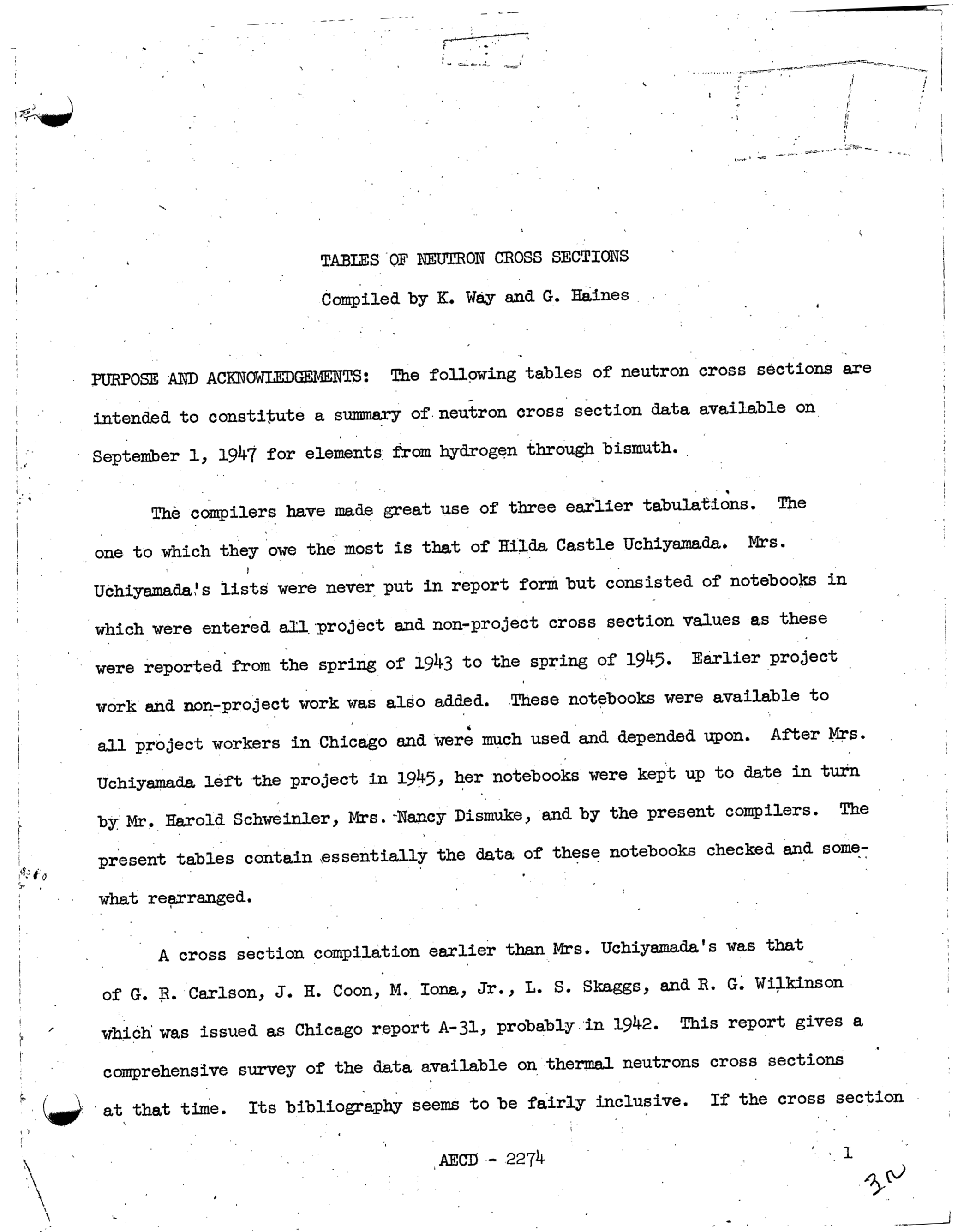


values given in all of its references were combined with those of the present tables, the resulting list would probably be a nearly complete one of all neutron cross-sections measurements ever made. It was not thought practical, however, to include all the results referred to by Carlson and his co-workers in the present tables. It seemed important to keep them from being too cumbersome and, therefore, to omit data which are no longer useful. The criteria used in deciding when to leave something out are described below.

Still a third compilation of neutron cross section data is contained in the graphs of H. H. Goldsmith and H. C. Ibser, MUC-HHG-\#7, which were first issued in August, 1945. This compilation is known to most as the wallpaper book and is referred to in the tables a.s WPB. It is understood that a revised edition, prepared by H. H. Goldsmith and B. T. Feld, will appear shortly in the REVIEWS OF MODERN PHYSICS. The present tables are meant to be used in conjunction with the old or new curves. The tables list all the papers which give data for curves and include in addition measured values of average cross sections which cannot be plotted. They can thus be used to check a given curve to see if it includes all pertinent data and for comparison of experimental averages with integrals over parts of the curve.

$\approx$ The present compilers received assistance not only from the three - earlier collections but also from other members of the laboratory. Mrs. Nancy Dismuke did some of the work of checking and tabulating and Mrs. I. I. Pogue did all of the work of arranging the material on the pages and typing the manuscript. Their help has been very much appreciated. Many others have generously given time - for discussion of certain experiments and have supplied references which had been 8 missed. 
CRITERIA FOR OMITIING MEASURIMINTS: As already mentioned not all knowa data has boen included in these tables. On the one hand the compllers did not went to intrude unduly their judgment in selecting data and on the other they did not want to make the tables unnecessarily largo and unwieldy. As a compromise the following principles were used as gutdes:

1. An experimenter's data was left out if poor agreement was found betreen some of his results and values which now seem fairly well established, and if in addition apparentily wore accurate nork was avallable for all the - lements or 1sotopes whose cross sections he measured. For Instance, all the data of Sinma and Yamasak1 (Phys. Rev. 29, 402 (1941) were omitted. Their activation cross section measurements for thermal neutrons show in general very poor agreement with values now considered to be reliable. (For instance they round 6 b for the as opposed to the accepted 13 b and 130 b for Au againgt the current $95 \mathrm{~b}_{\text {.) }}$ In addition the activation eross sections of all the isotopes studied by Sinma and Yamabakt have been remeasured by $\mathcal{L}$. Seren and many of thetu by other experimenters who are in fair agreenent with Seren.

The early tranomission results of J. R. Dunning, G. B. Pegram, G. A. F1nk, D. P. Mitchel1 (Phys. Rev. 48. 265 (1935)) have, however, beén included. Although these data have been auperseded in many sases by work in which better geometry, etc. wased, nevertheless in many other cases they are one of only two or three measurements about all of which there is st111 a good deal of doubt.

2. In the case of single measurements, early results were left out only in cases where there seemed to be overwhelning evidence that they must have been in error. For example, the early values of the hydrogen cross section at indive resonance were onitted. This quantity was originally thought to be $\sim 13$ b but now a value of $\sim 21$ b is generally adopted. 


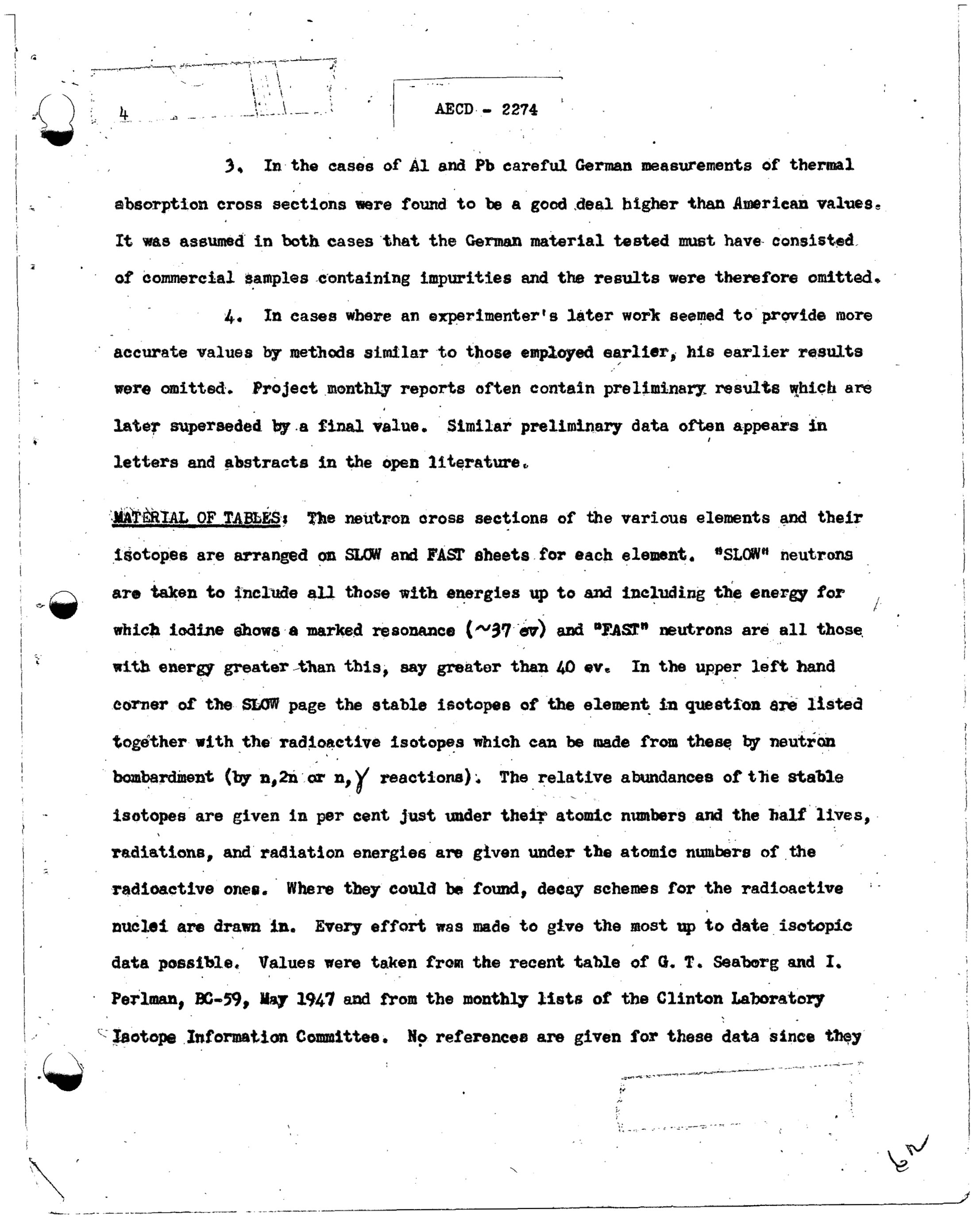


are included chiefly for orlentation purposes. References are given, however, for the deeay sohemes which might be otherwise a ilttle difficult to locate.

Cross sections are divided into total, scattering, and abserption cross sections. Fótal eross sections, the sum of absorption plus all scattering eross sections, which are measured by transmission methods are given first. They are followed by scattering cross sections which are always for solid angles of $4 \pi$. Where the scattering has been measured at some particular angle and this value assumed to hold for other angles, this fact is noted in the reference sheets. Following the regular reference list is an additional one for material on differential, inelastic and transport cross sections which could not be systematised in time for inciusion in the main tables. Onder absorption are given first the cross section values for the entire element and below those for individual isotopes when these are known. Activation eross sections for $n \gamma$ reactions ore marked with the half-life of the activity produced. The cross sections for np and $n \alpha$ reactions follow symbols such as $\mathrm{s}^{32}$ tap, etc.

All cross sections are given per atou of element unless othervise Indicated of a symbol for a molecule or isotope in the extrene left hand columa.

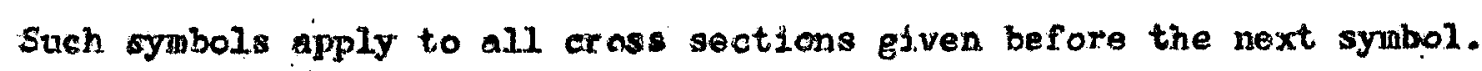

The column headed "Source" describes the neutron source by means of symbols which are explained on the abbreviation page.

The symbols or figures in the column headed "Heutron Energg" are supposed to give the average or effective energy of the neutrons detected in the oxperilant. The symbols and other conventions used are again explained on the abbreviation page. 


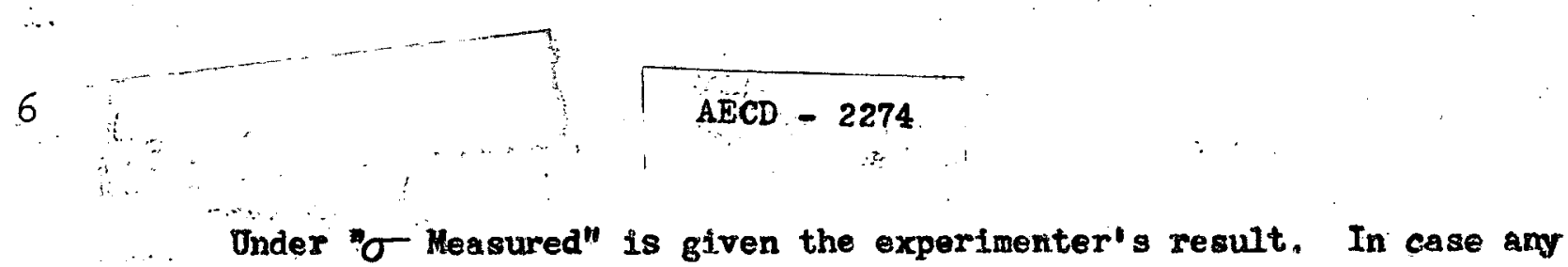

correctione or changes have been made by the compllers because of changes in "standard" values or for other ressons, this fact is noted either in the main - Eable or under the appropriate reference.

At first it was intended to include the experimenter's limits of 0

error: As the rork of compliation progressed, however, it became ovident that usually these lintts of error applied only to reproducibility of the result of the particular method used and did not give a measure of the error in the absolute value of the cross section. It was then decided to omit almost all measures of error but a number had already been included so that the result is that no consistent polley in this matter has been followed on all the sheets.

The SIOW page contains a speolal column for the value of " $\sigma$ at .025 ev." (This neutron energy is taken to correspond to a neutron velocity of $2200 \mathrm{~m} / \mathrm{s}_{\text {.) }}$ Often the experimenter calculated from his data the cross section to be expected for monochromatic neutrons of this energ ssiuming the cross section in question to vary as $\mathrm{l} / \mathrm{v}$. In some cases in which the experimenter did not do this, the compllers have made an estimate of the .025 ev cross sectlon by Iinding the fector by which one of the experimenter's values must be multiplied to give .025 . cross section that is fairly well established and then multiplying all the other results of this partieular experimenter by this same factor. Factors used and the cross sections on which they are besed are given in a note after the appropriate reference.

At the top of the FAST page are given values for absorption resonance energles, the cross eections at these energies and the whole wldthe: of the line where this is known. The recent data on scattering resonances was not included. Here again this materfal is intended fust for purposes of orfentation and no references have been givera. 


\section{ABBREVIATIONS}

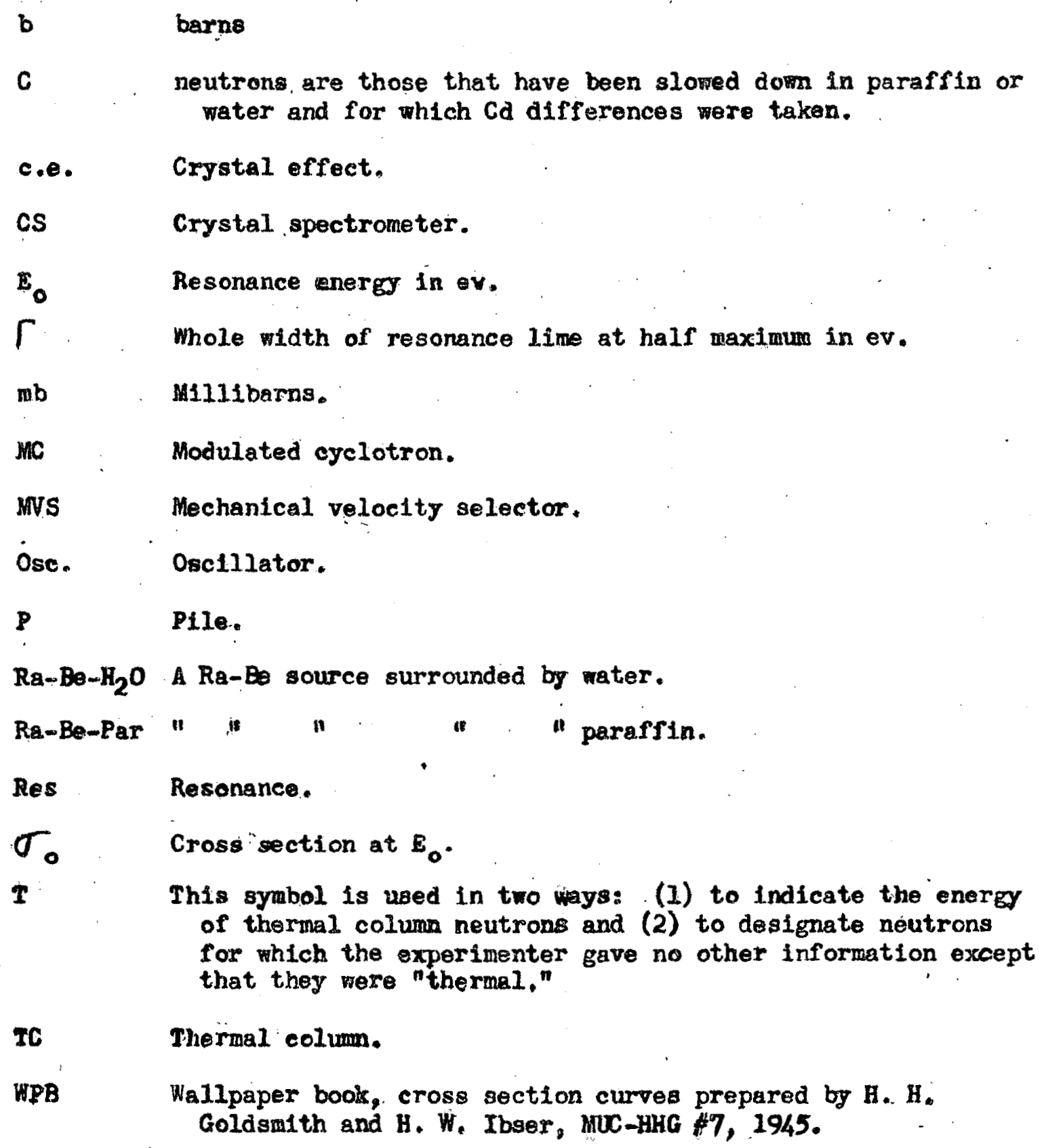
of thermal column neutrons and (2) to designate neutrons for which the experimenter gave no other information except that they were "thermal,"

TC Thermal colum.

WPB Wallpaper book, cross section currea prepared by H. H. Goldsalth and H. W. Ibser, MUC-HHG \$7, 1945.

EATERGIES OF PHOTO MEUTRONS: The energfes of neutrons coming from photo sources have been assigned 'standard' values irrespective of the energies 
attributed to them by different experimenters. Pertinent data on $\gamma$ ray and neutron energles were collected in a memorandun by Hancy Dismuke and $\mathrm{K}$, Way (CLM-KW-5) and used to assiga 'best valuea' to the energies wich photo neutrons would have if there were no moderation in the source. The ralues used in the tables are these values reduced by $20 \%$ to allow for degradation. This figure of $20 \%$ was chosen somewhat arbitrar11y. Most experimenters do not make ang estimate of the energy degradation; Different amounts will certainly occur in differently construoted aources so the actual values of the energies of the different photo neutrons used may be $10 \%$ higher or lower than the value given in the tables even if the 'best' thin source value was a correct choice.

\begin{tabular}{|c|c|c|c|}
\hline Source & $\begin{array}{l}\text { Heutron Energ if } \\
\text { No Moderation in Source }\end{array}$ & $\begin{array}{l}\text { Neutron Enorg } \\
20 \% \text { Loderation }\end{array}$ & $\begin{array}{l}\text { if } \\
\text { in source" }\end{array}$ \\
\hline $\mathrm{Sb}-\mathrm{Be}$ & 0.029 & 0.023 & \\
\hline $\operatorname{Ra-Be}$ & $\begin{array}{l}0.12 \\
0.51\end{array}$ & $\begin{array}{l}0.09 \\
0.4\end{array}$ & \\
\hline $2 a-D_{2} 0$ & 0.16 & 0.13 & 8 \\
\hline $\mathrm{Th}-\mathrm{D}_{2} \mathrm{O}$ & 0.22 & 0.18 & \\
\hline$Y \cdot B \boldsymbol{B}$ & 0.22 & 0.18 & \\
\hline $\mathrm{Na}-\mathrm{D}_{2} \mathrm{O}$ & 0.29 & 0.23 & \\
\hline$L a-B_{\theta}$ & 0.77 & 0.62 & \\
\hline $\mathbf{M}-\mathrm{BA}_{\mathbf{B}}$ & 0,88 & 0.70 & \\
\hline $\mathrm{Ma}-\mathrm{Be}$ & 1.00 & 0.80 & \\
\hline
\end{tabular}

* Sxan CLM-KiN-5.

* A Y ray with 2.42 mev energy 18 given by Latyshev, Rev. Hod. Phys. 19, 132 (1947). The relative intensities of the photo neutron group is not known. 
OAHER HETTROU EUERGIES: The energy of a Ra- $\alpha$-Be source has been given rather axbitrarily in the tables as $\sim 4$ mev, A recent paper by D. L. Hill (CP-3800) indicates, however, that tho Ra- $Q$-Be spectrun has a miaxim at $\sim 5$ mev with an average energy of 4.4 mev, Dewers (MP-204) found a maximum at $\sim 5$ mev also but an average of 5.3 me. In general for other sources, the experimenter's estimate of the mean or effective neutron energy has been used. 
SLON (continued)

Hydrogen
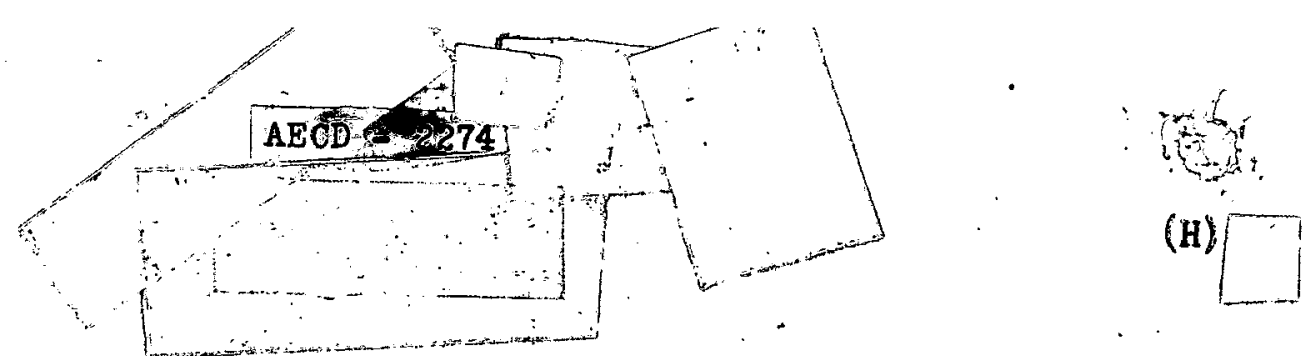

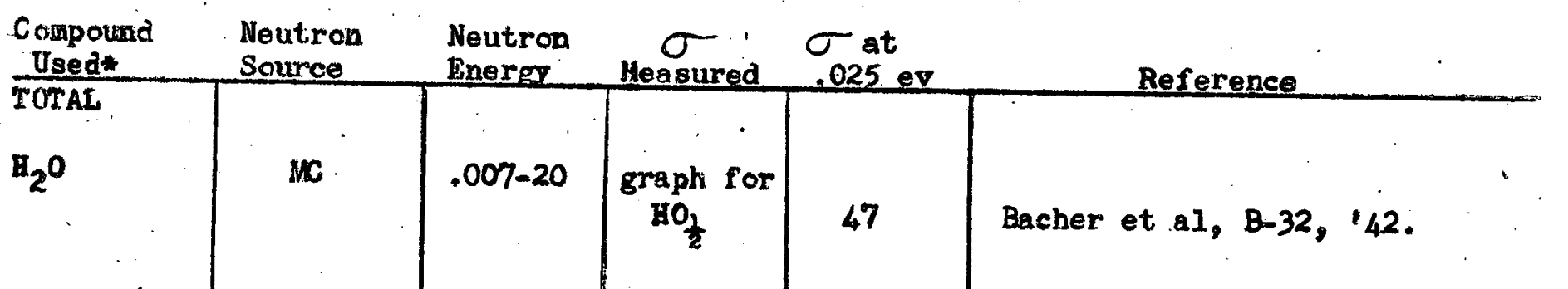

Values of $\sigma_{t}$ for ortho- and para-hydrogen at room and liquid air temperatures are given by Brickpedde et al, B-2 (liquid) and by Libby and Long, I-26, (gas). Los Alamos work will bo published soon.

For cross sections of $\mathrm{H}$ in other hydrogeneous compounds, see Carroll, C-3; Libby and Long, L-26; and CarIson, C-2.

\begin{tabular}{|c|c|c|c|c|c|}
\hline ABSORPT IOA & $\overbrace{\text { D-D }}^{\text {Rn-BenPar }}$ & $\begin{array}{l}\mathbf{C} \\
\mathbf{T}\end{array}$ & .25 & $\begin{array}{l}.33 \\
.33\end{array}$ & $\begin{array}{l}\text { Vols, } \mathrm{V}-1,1,43 . \\
\text { Manley et } a 1, M-22,142 \text {. }\end{array}$ \\
\hline ABSORFI IOA & $\begin{array}{l}\text { RATIOS fron } \\
\text { Elements } \\
\text { LI/A } \\
\text { B/A }\end{array}$ & $\begin{array}{l}\text { integrated } \\
\begin{array}{|c}\text { Ratio } \\
242.4 \\
1940 \\
1954 \\
2160 \\
2380 \\
2270 \\
2270 \\
44.2 \\
1.0 \times 10^{4}\end{array}\end{array}$ & 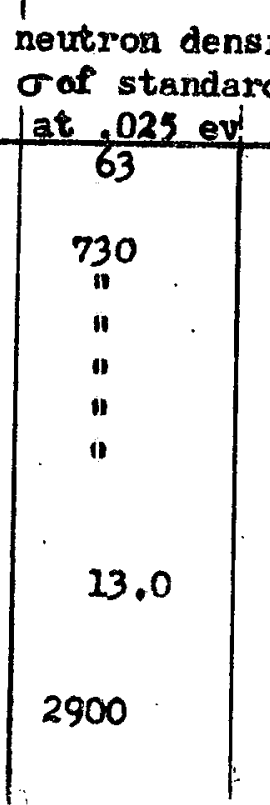 & $\begin{array}{c}\text { tios in d } \\
\sigma \text { at } \\
.025 \mathrm{ev} \\
.26 \\
.38 \\
.37 \\
.34 \\
.31 \\
.32 \\
.32 \\
.29 \\
.29\end{array}$ & 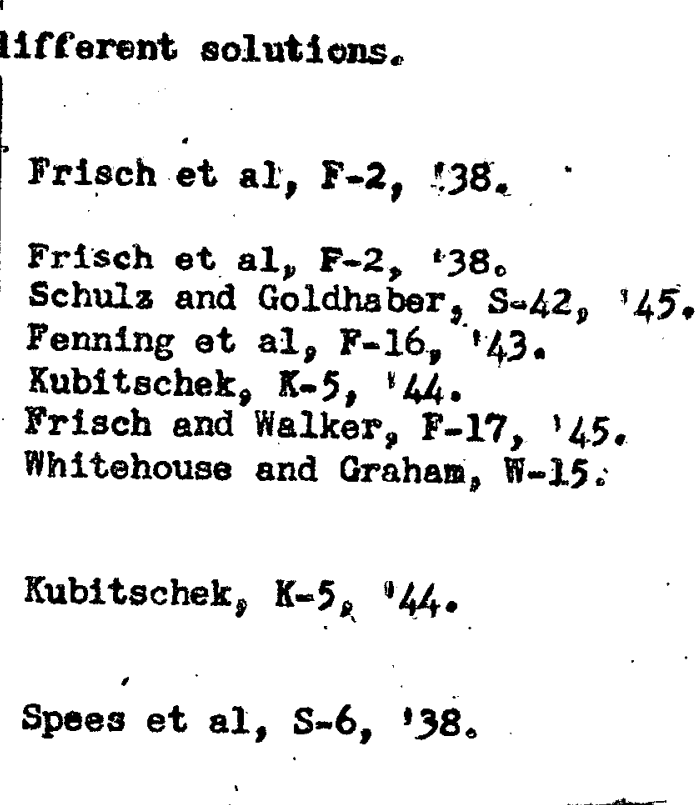 \\
\hline
\end{tabular}

Por diffusion length experiments sea $s . \bar{k}$. Allison et a.l, Report $\mathrm{G}$. 82 and 7. C. W1180n, CP-2906 Gamertafelder and Goldhaber, Phys. Revi, 62, 556. (1942).

* All cross sections on this page are for the hydrogen aton.
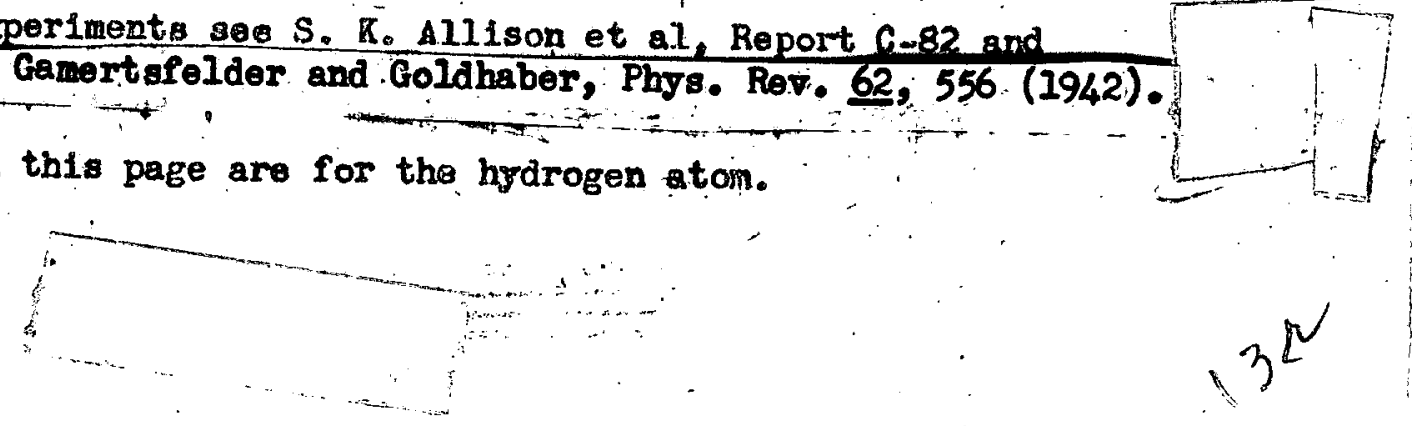


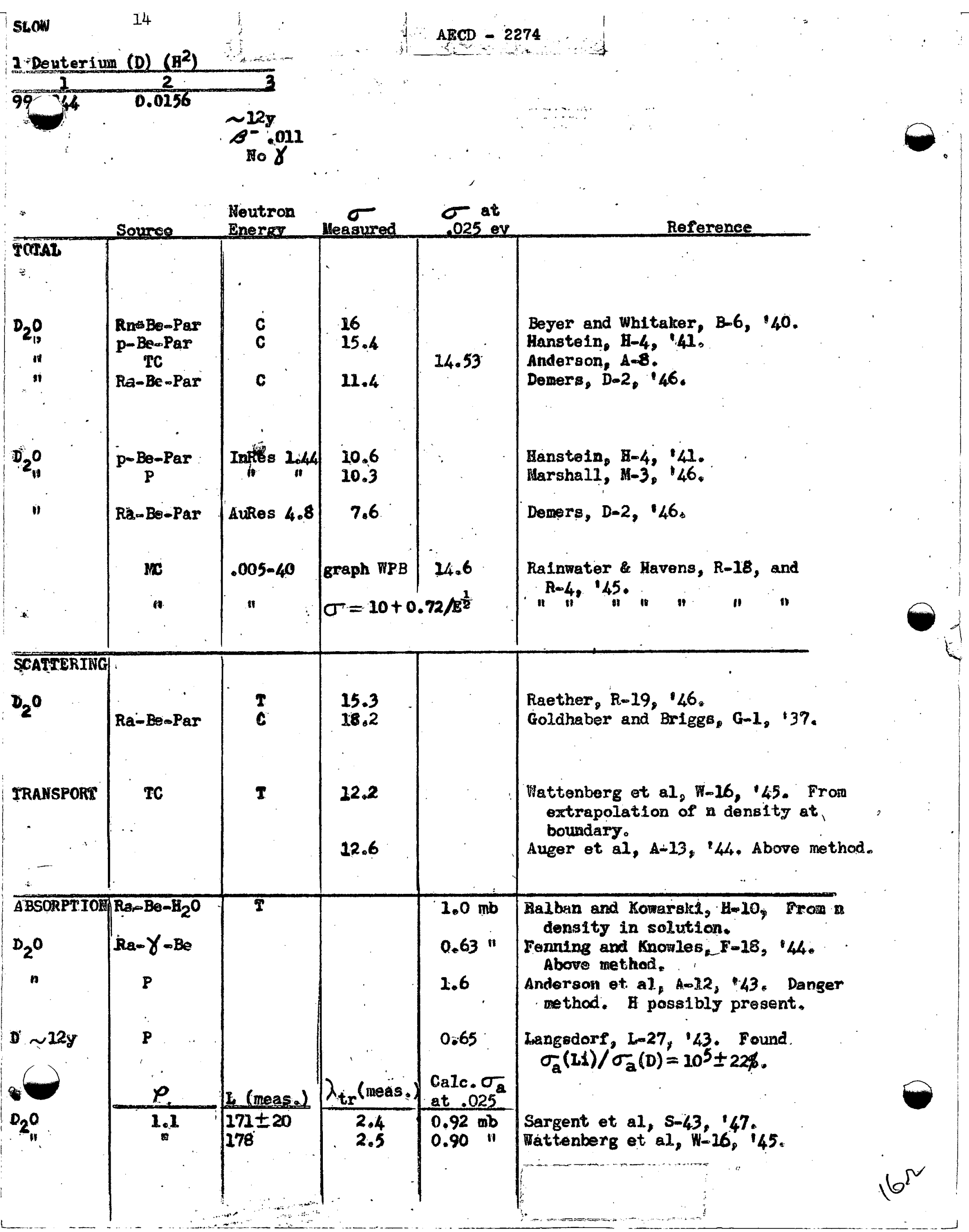


FAST

Deuterium

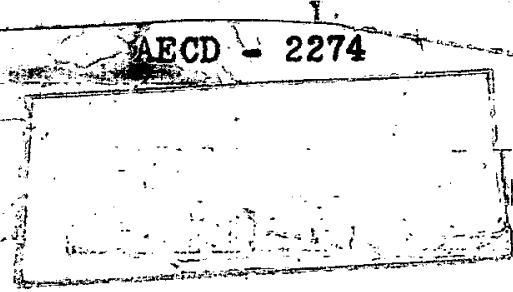

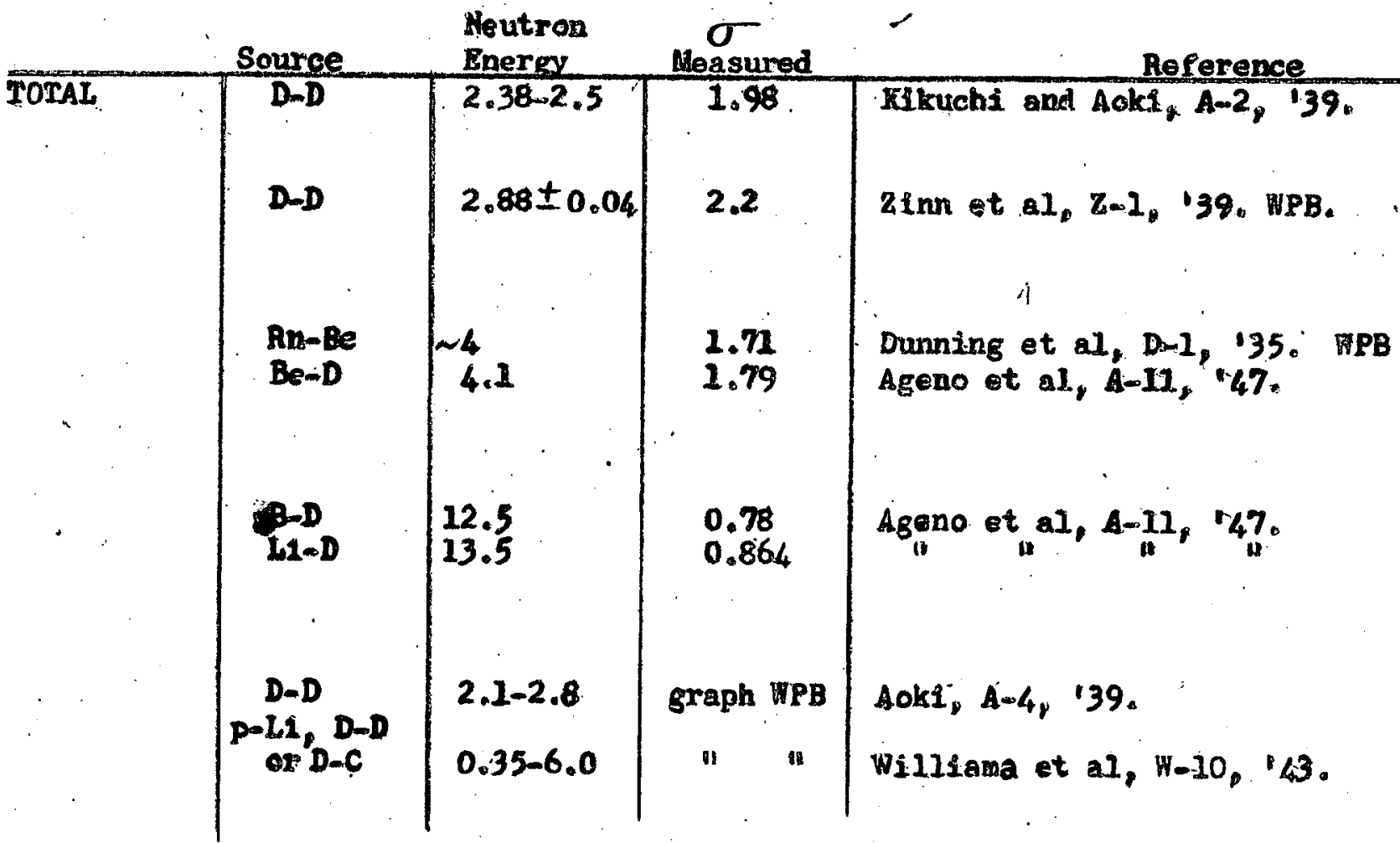

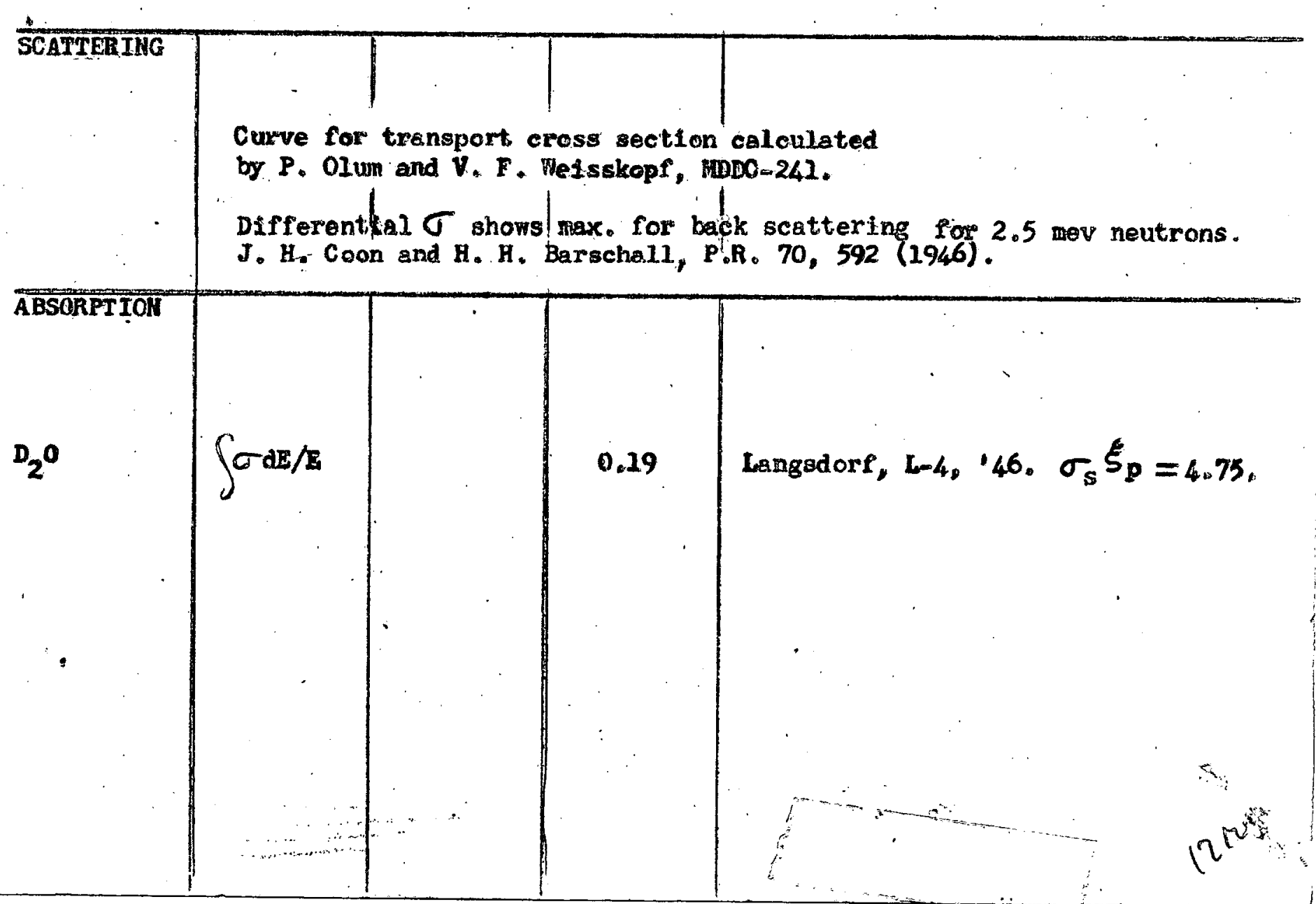




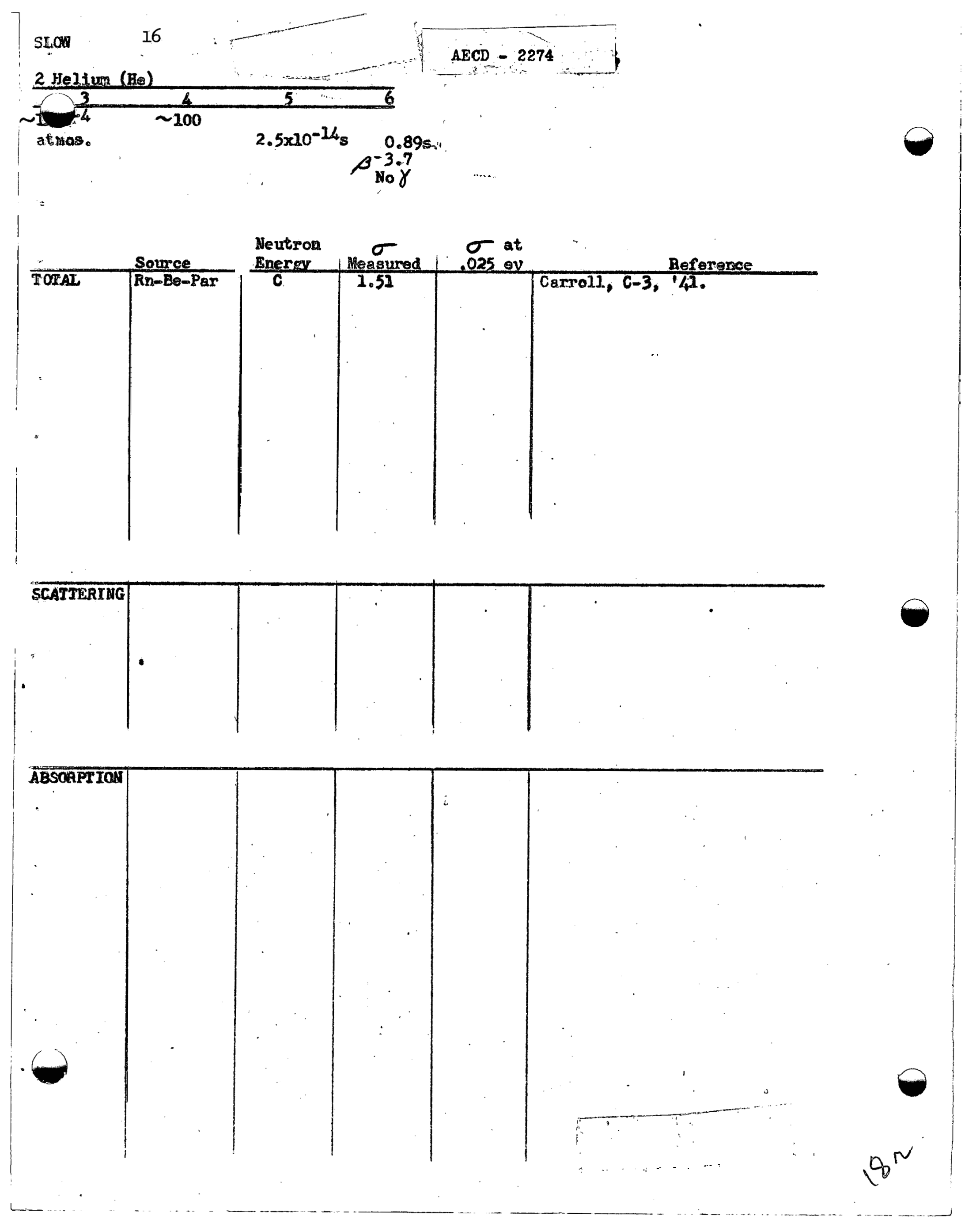




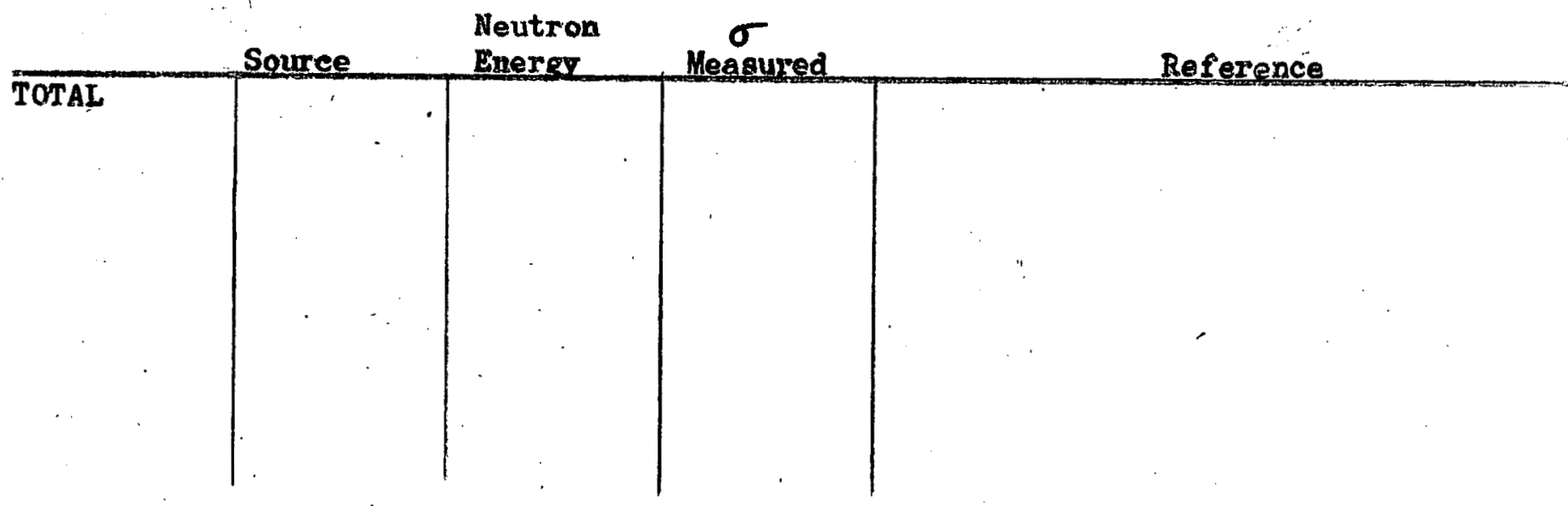

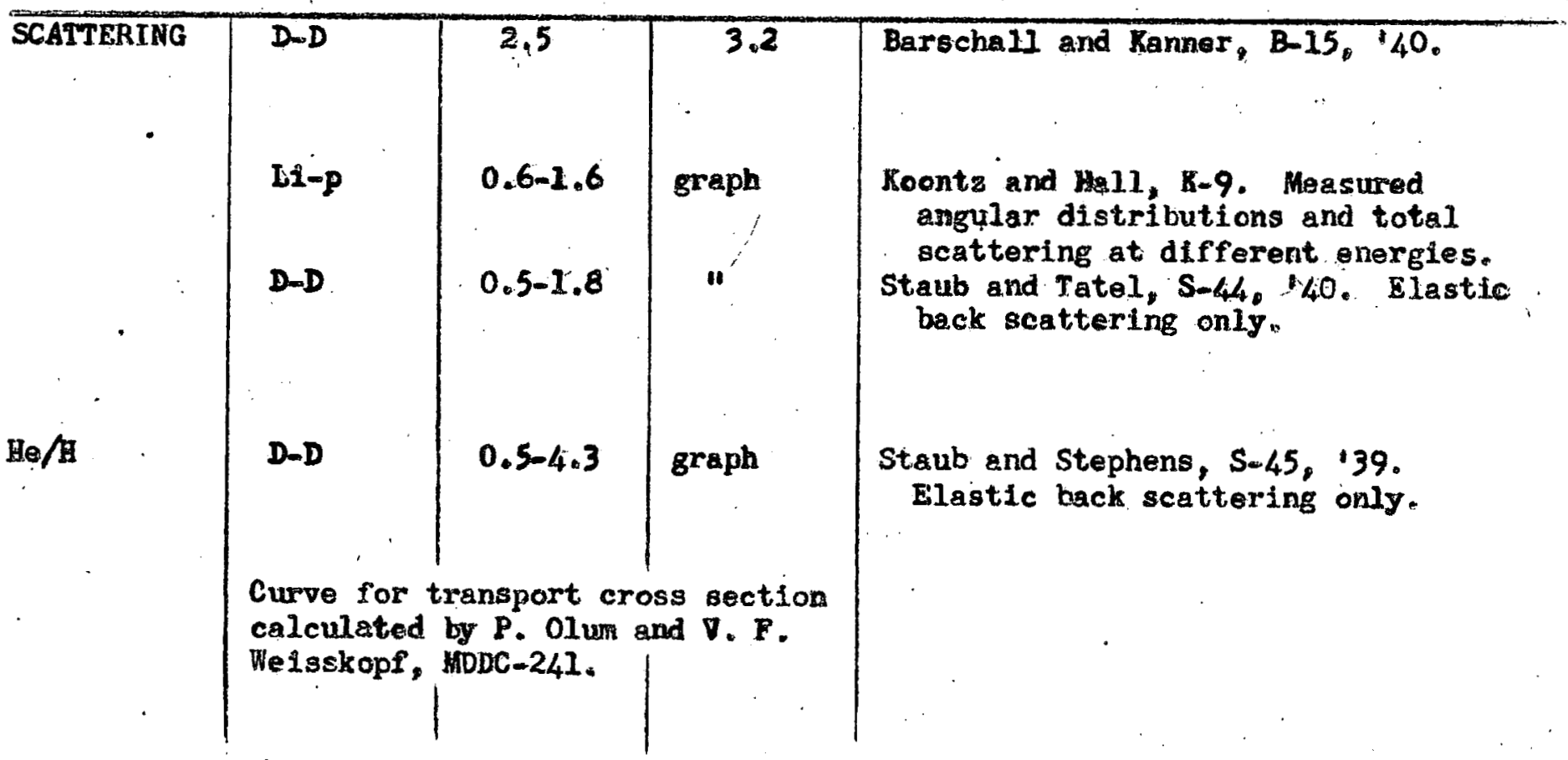

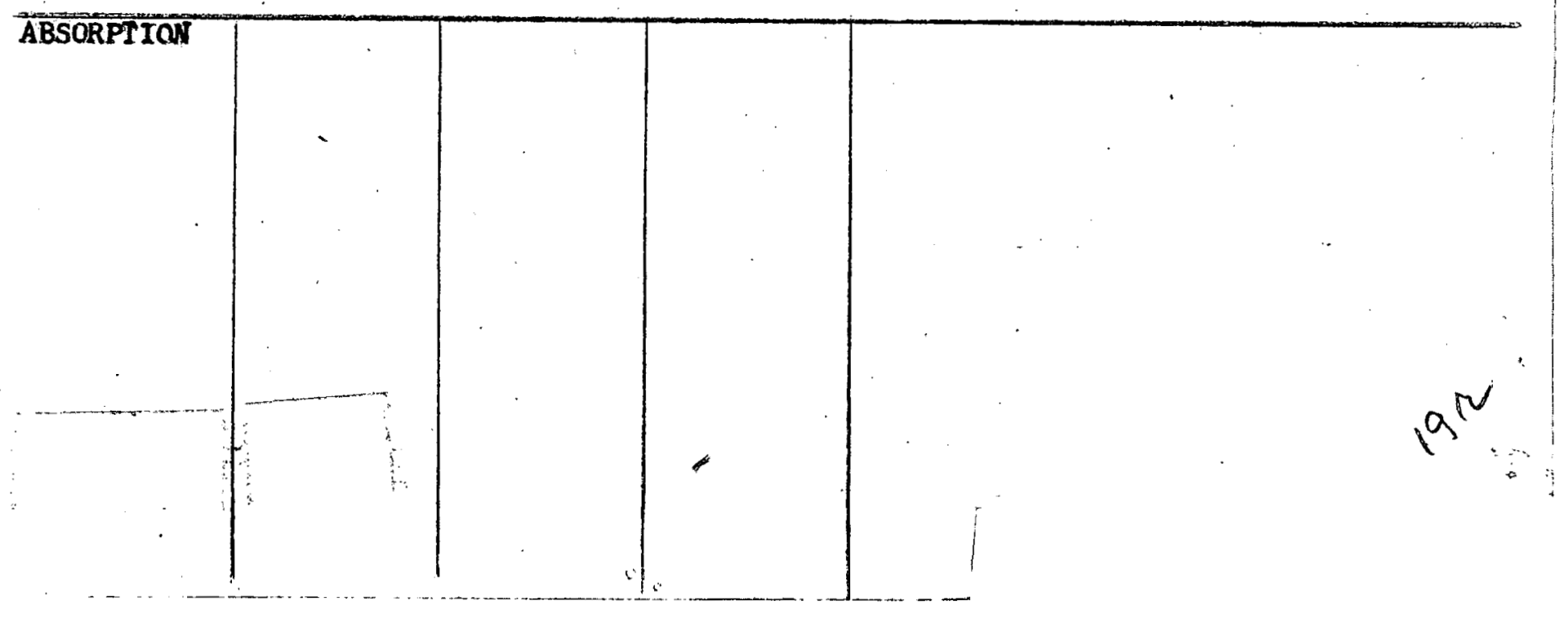




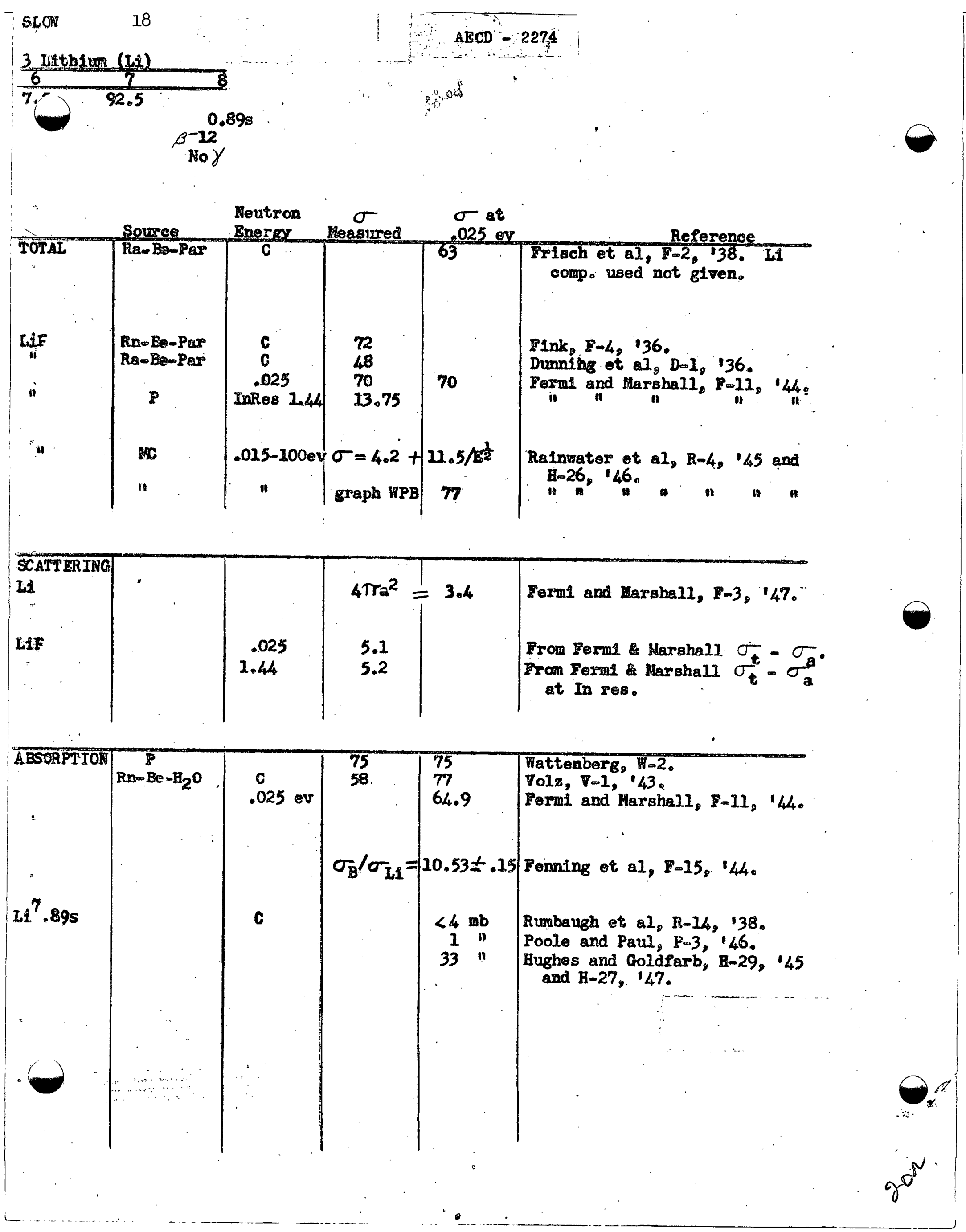




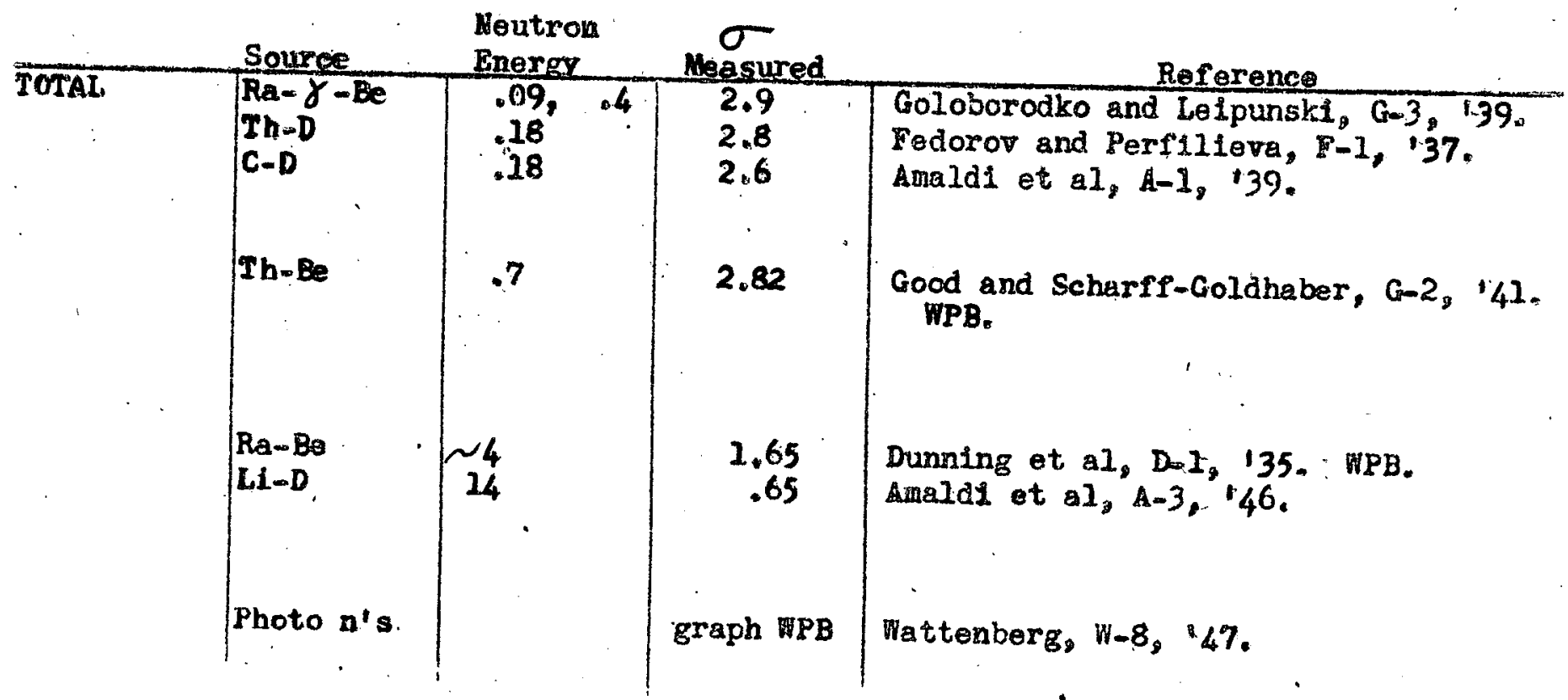
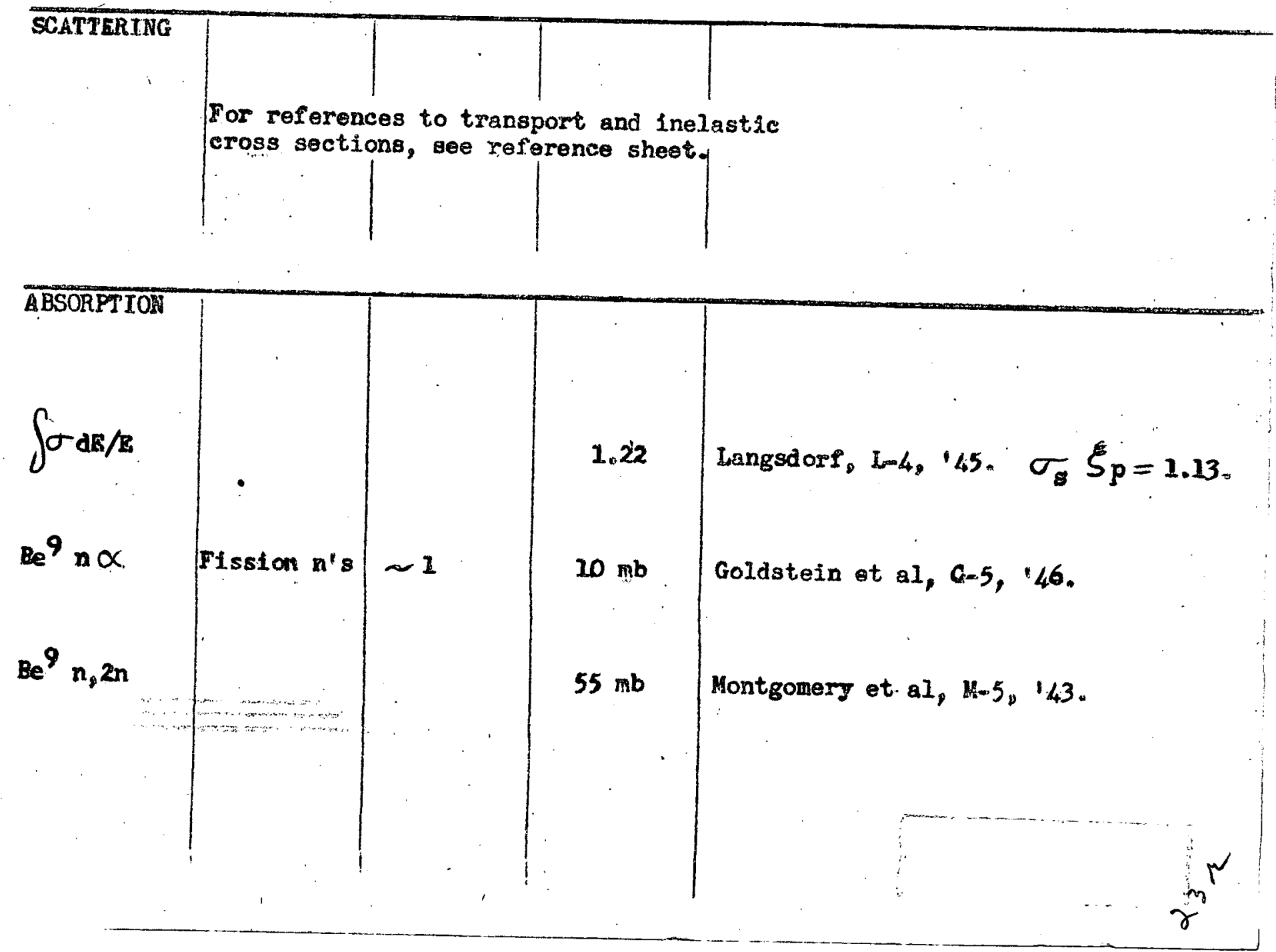


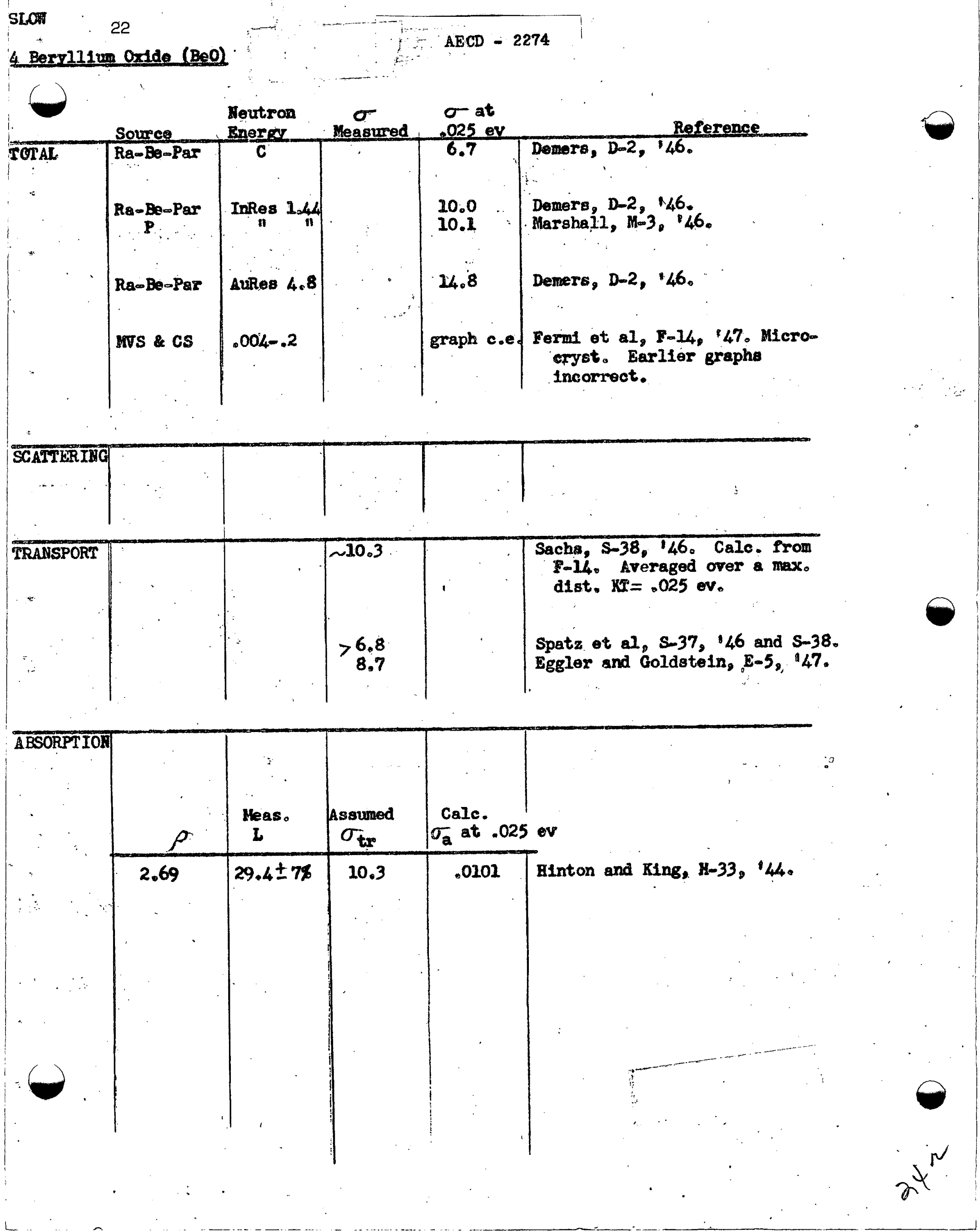




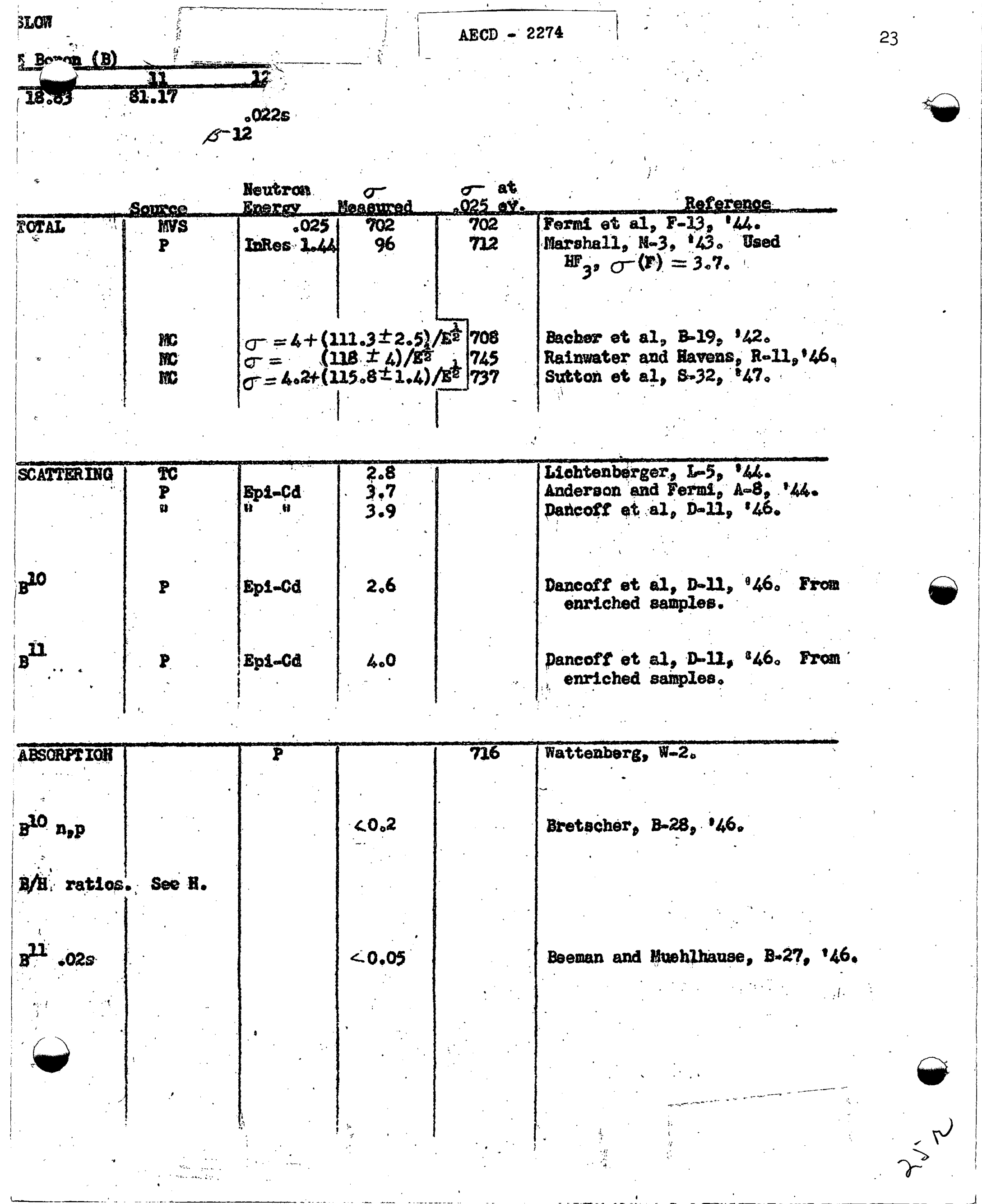




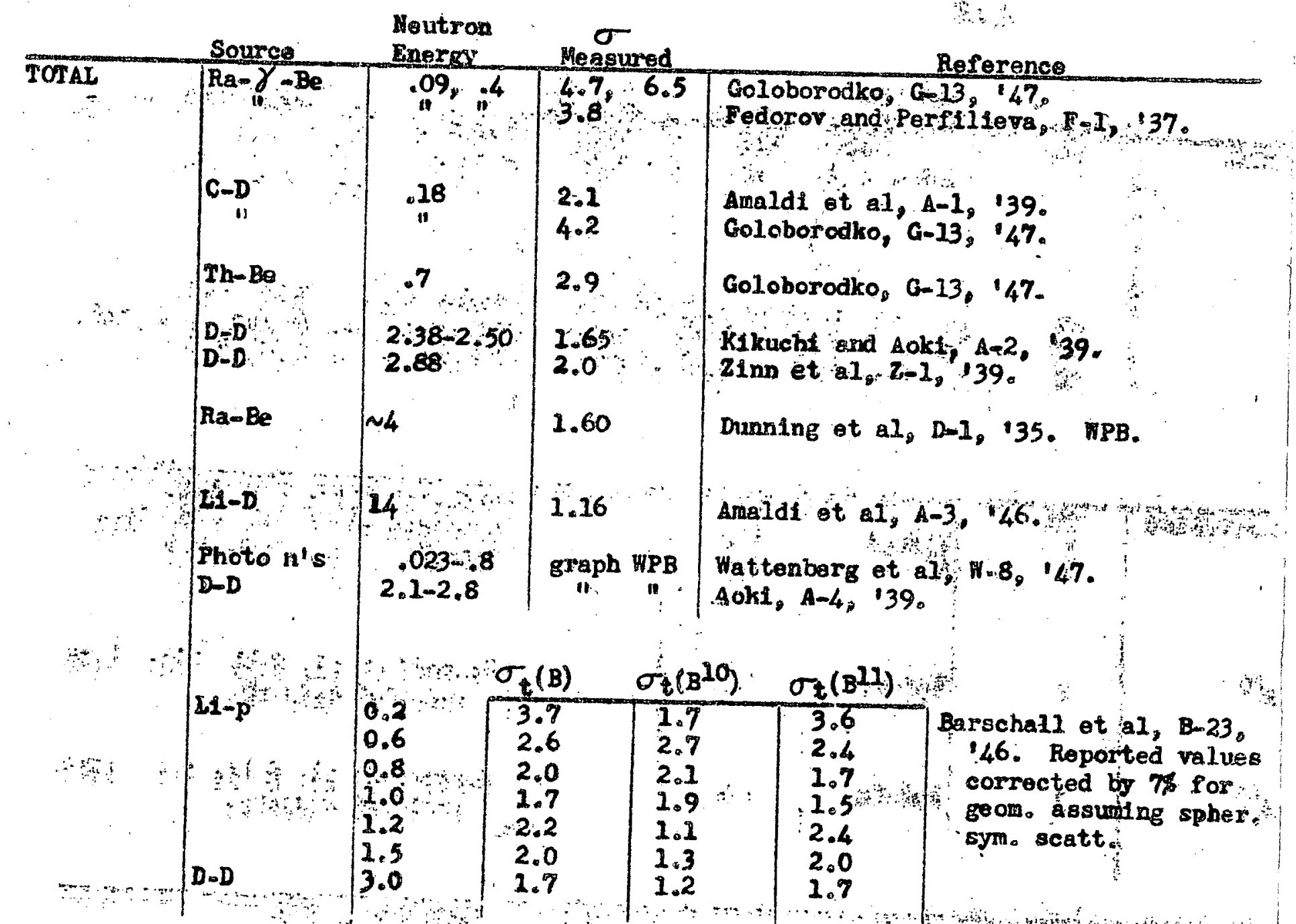
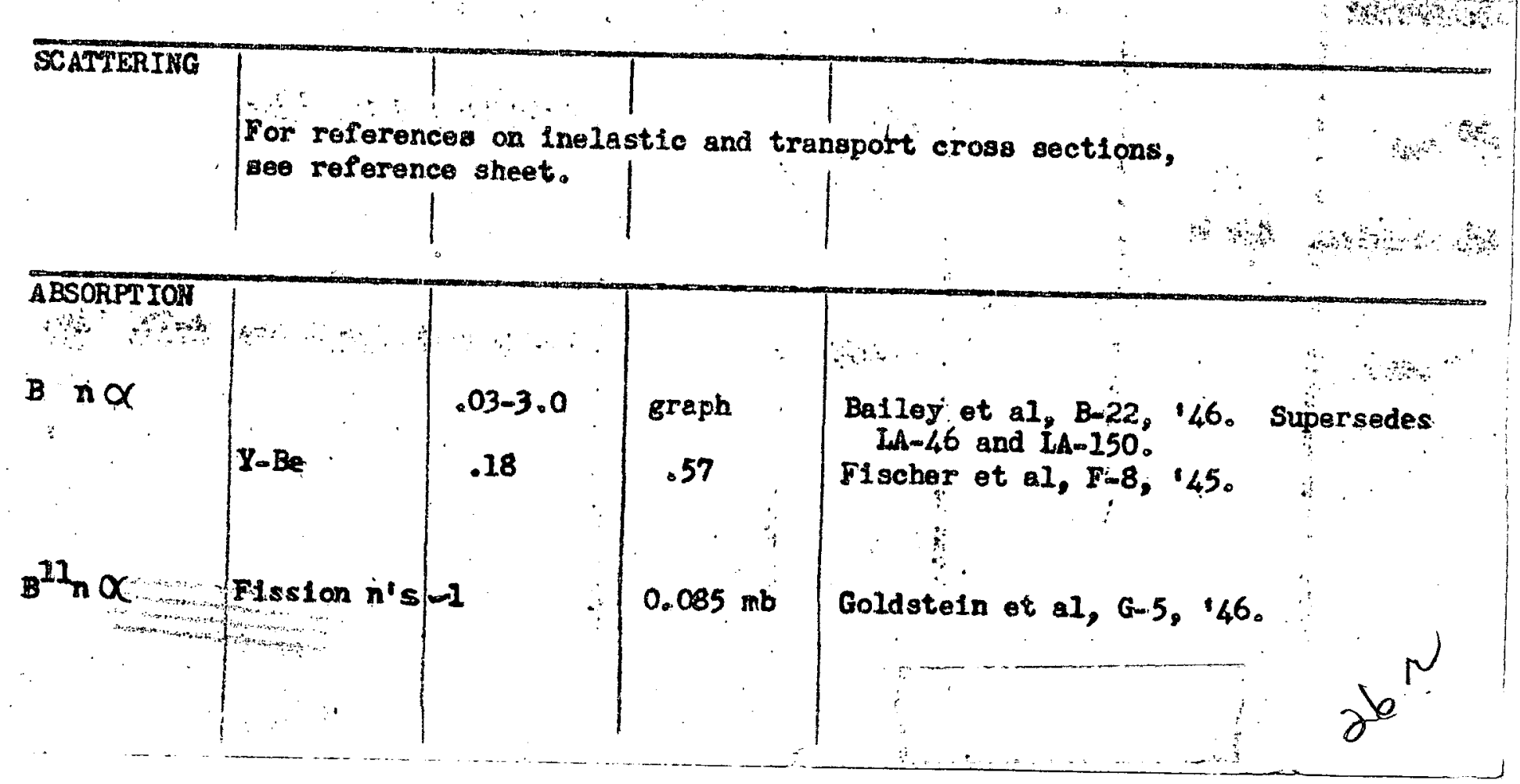


\section{Carbon (C)}

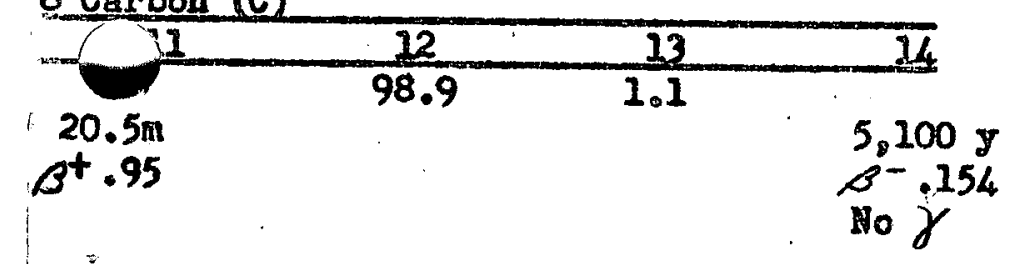

Goldhaber and Briggs, G-1, 37 .

Beyer and Whitaker, $B-6 ;{ }^{4} 0$.

Hanstein, $\mathrm{H}=4, \mathrm{H} / \mathrm{H}$.

Whitaker and Bright, 1,142 .

Carlson et al, C-2.

Hanstein, $\mathrm{H}-4, P$, 41 . WPB。

Marsha11, M-3, 1/43. MPB.

Rainwater et al, $\mathrm{R}-4,145$.

Sturm, S-14, 146. Graphs for

Irrad. and unirrad. samples.

Weber and Arnold, W-i2, 147 .

Graphs for extruded sample.

Sachs and Goldberter, S-34, 147 .

Formd and Marshall, $7-5,47$.

Fermi and Marshall, F-5, 147. $\lambda=5.2 \mathrm{~A}^{\circ}$. 
SLOW (Continued)

6 Carbon (C)

\begin{tabular}{|c|c|c|c|c|c|}
\hline & $\begin{array}{l}\text { Neutron } \\
\text { Source } \\
\end{array}$ & $\begin{array}{l}\text { Neutron } \\
\text { Energy }\end{array}$ & $\begin{array}{c}\sigma \\
\text { Meesured }\end{array}$ & $\begin{array}{c}\sigma \text { at } \\
.025 \text { ev }\end{array}$ & Reference \\
\hline SCATTERING & & & & & \\
\hline TRANSPORT & & & 4,5 & & Anderson, $A-14,1 / 2$. \\
\hline
\end{tabular}

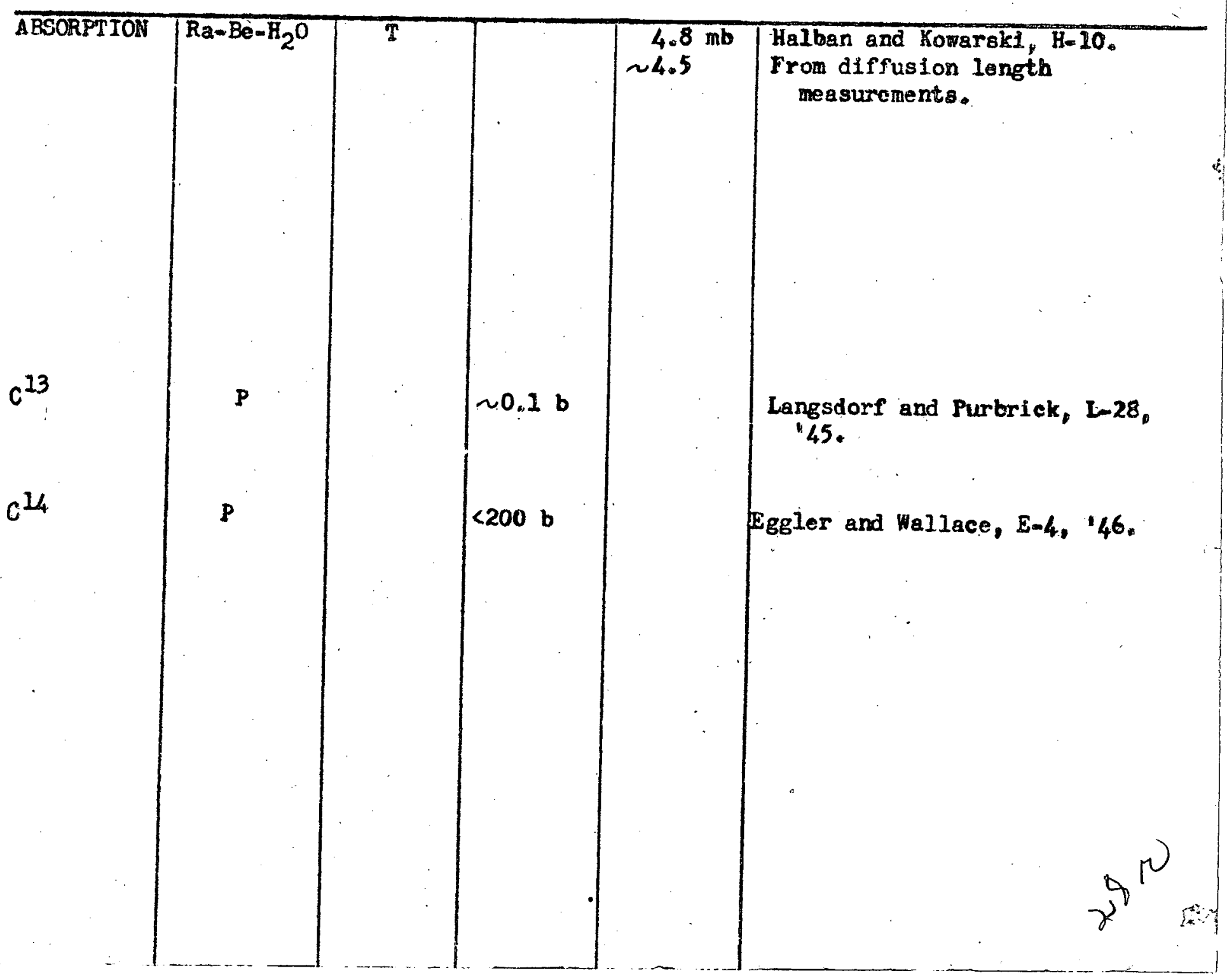




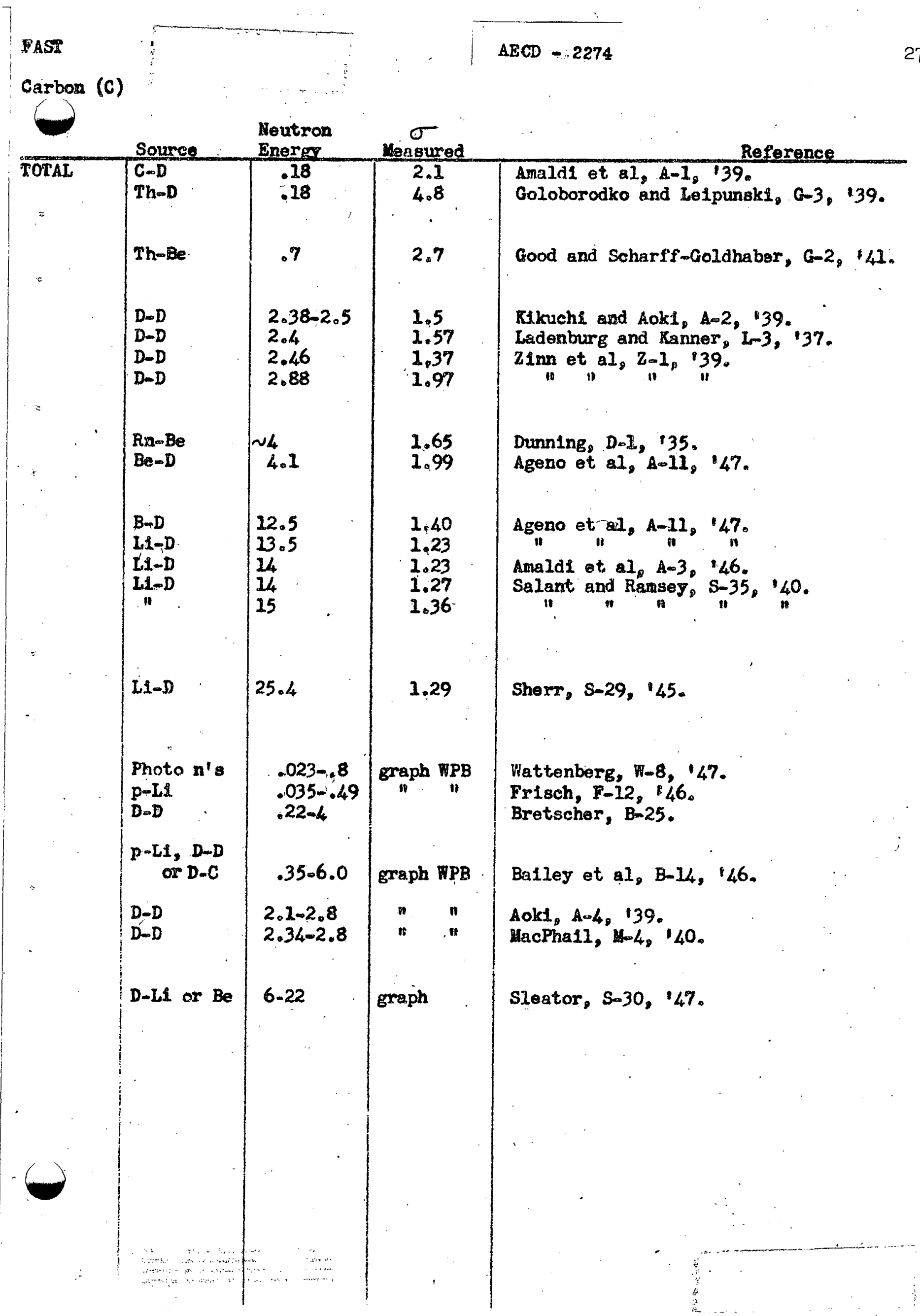




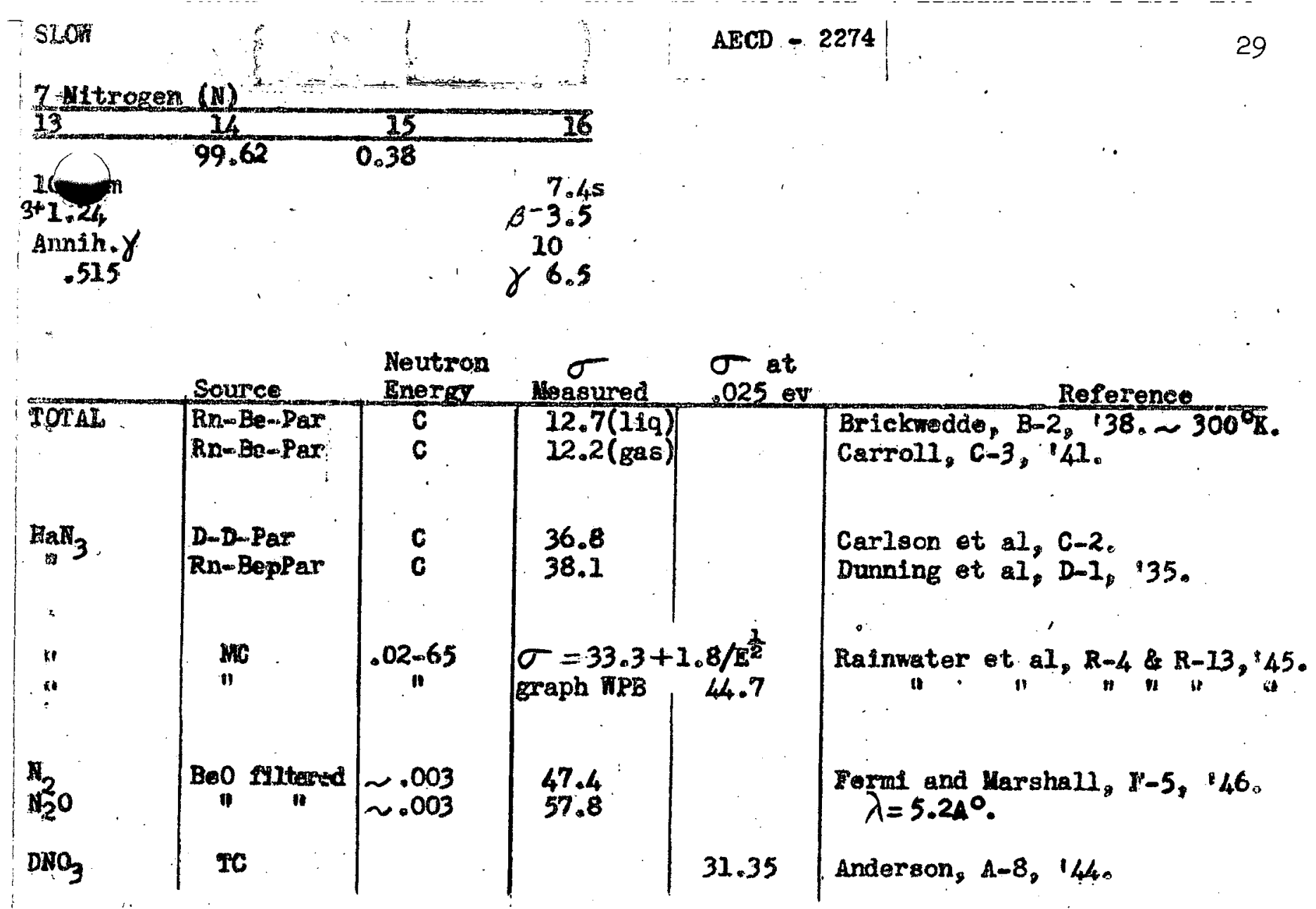
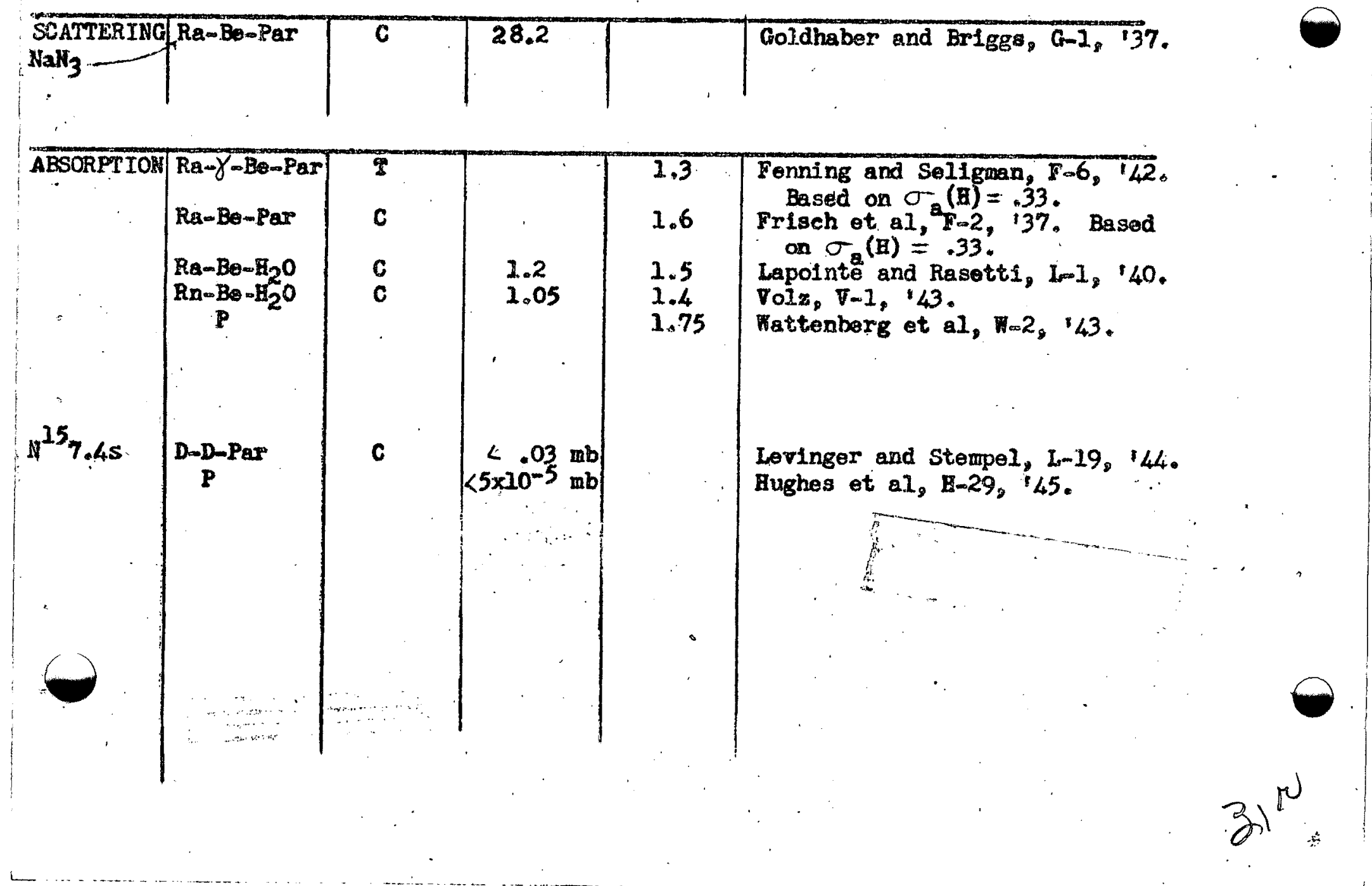


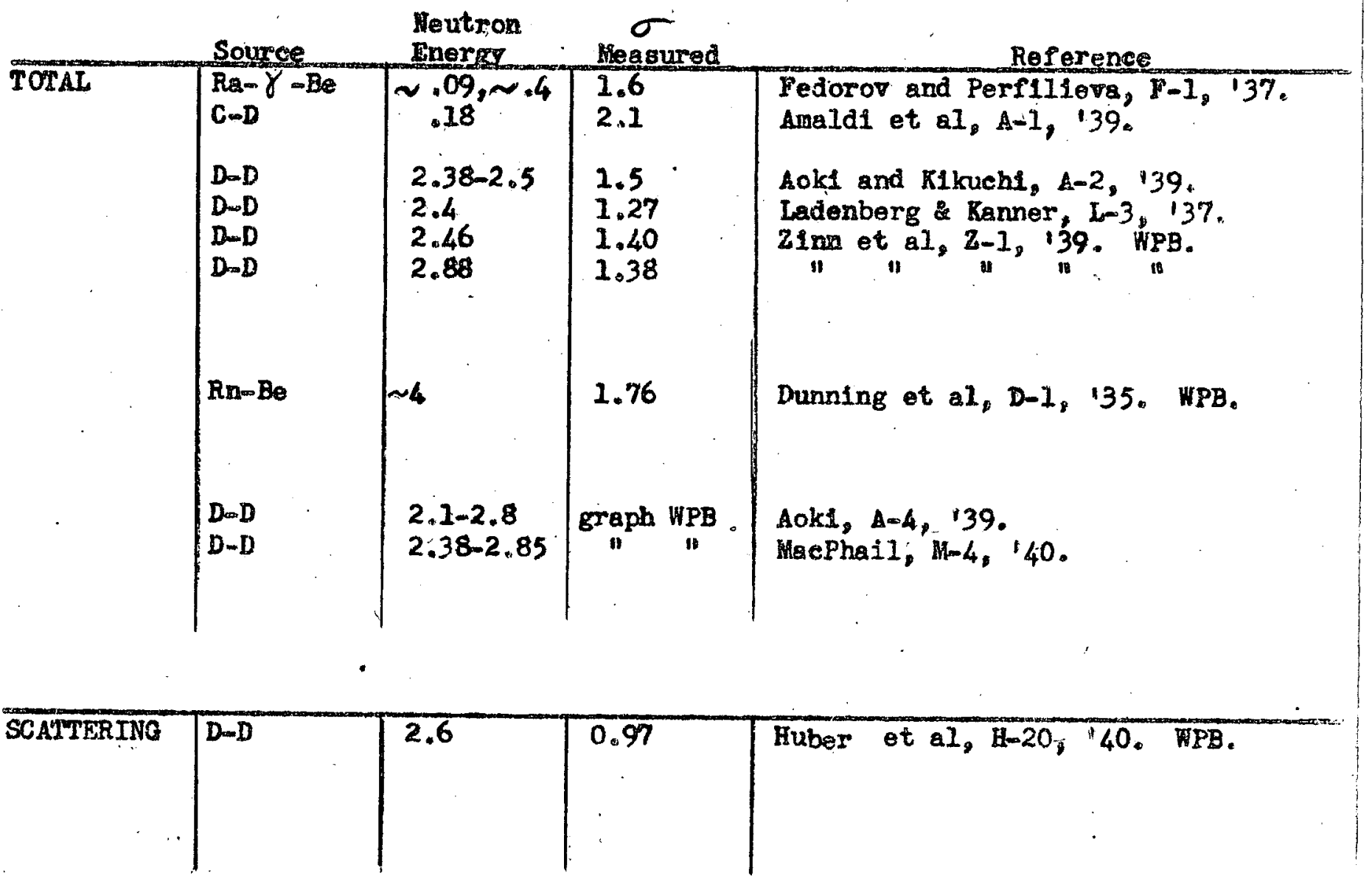

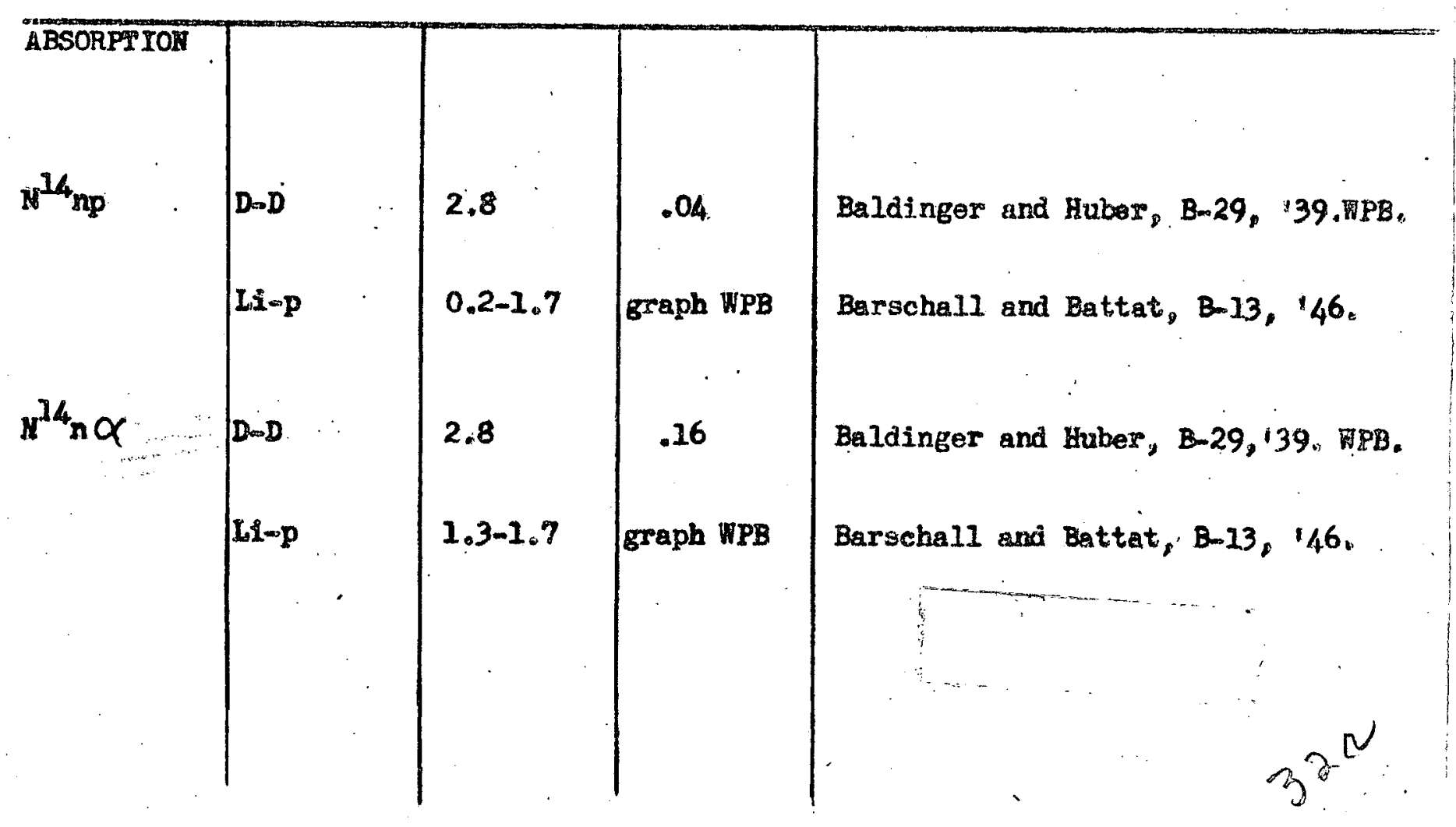


$8 \times 0 x y e n(0)$
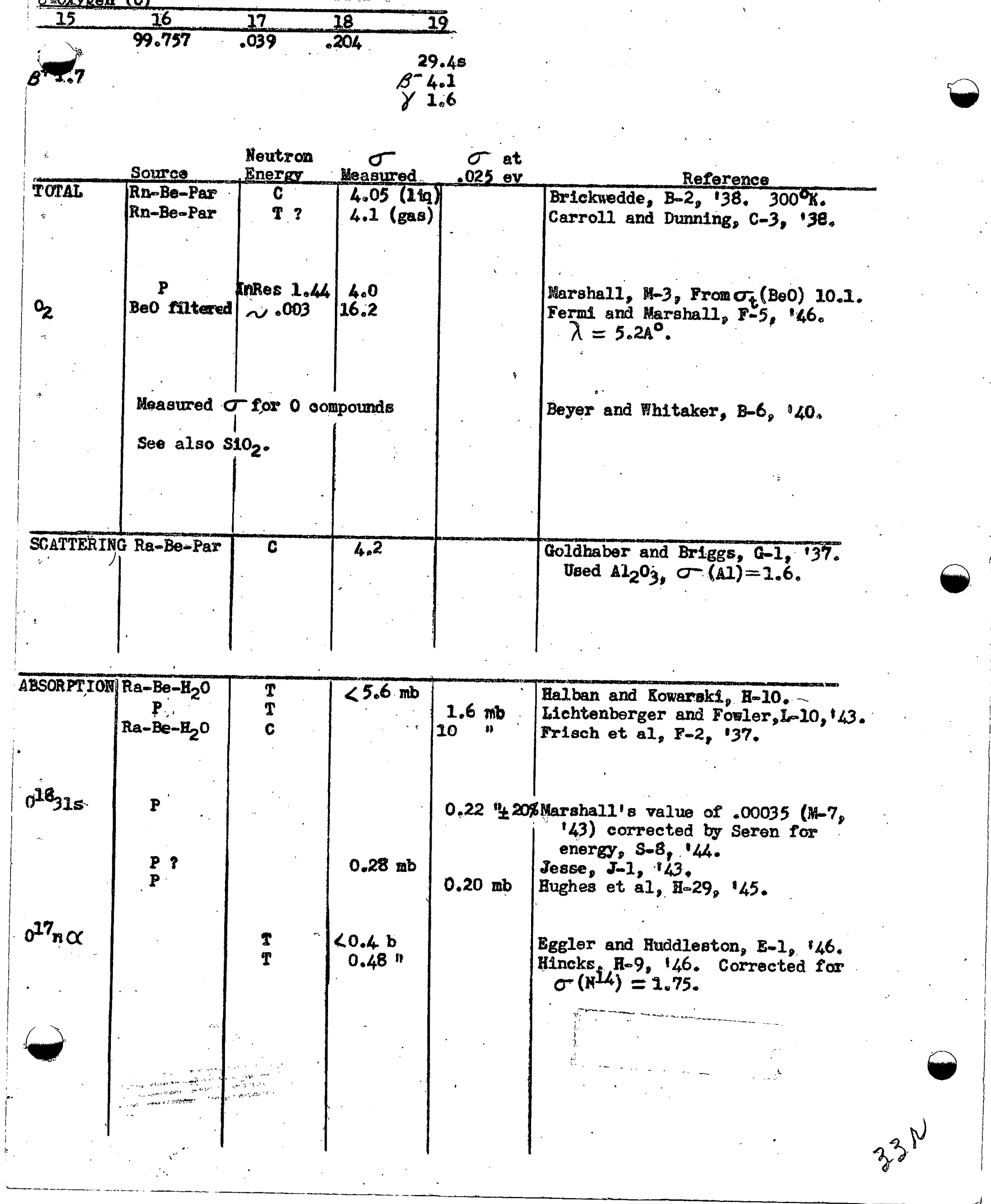
Oxggen

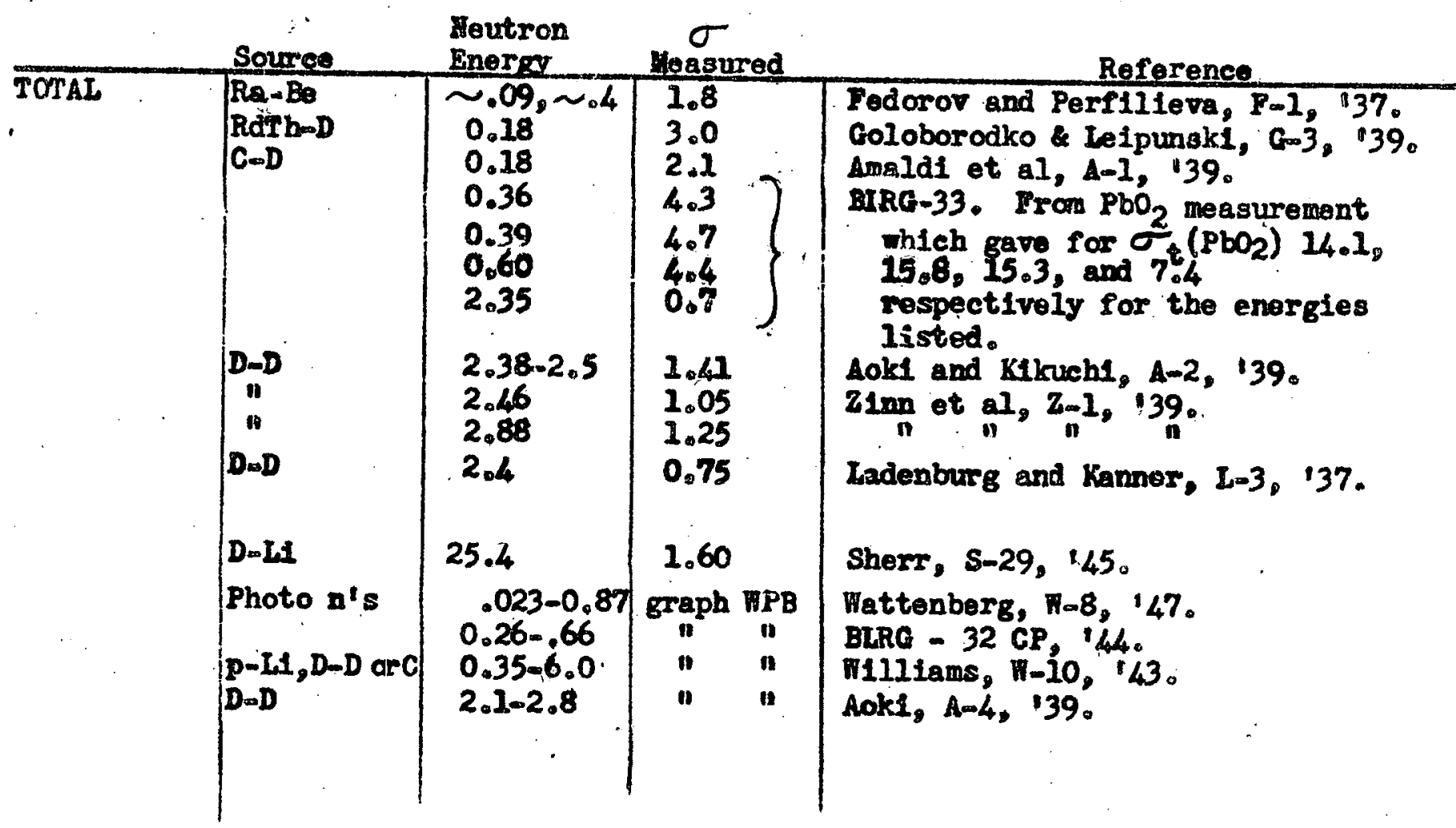

SCATIERIRG

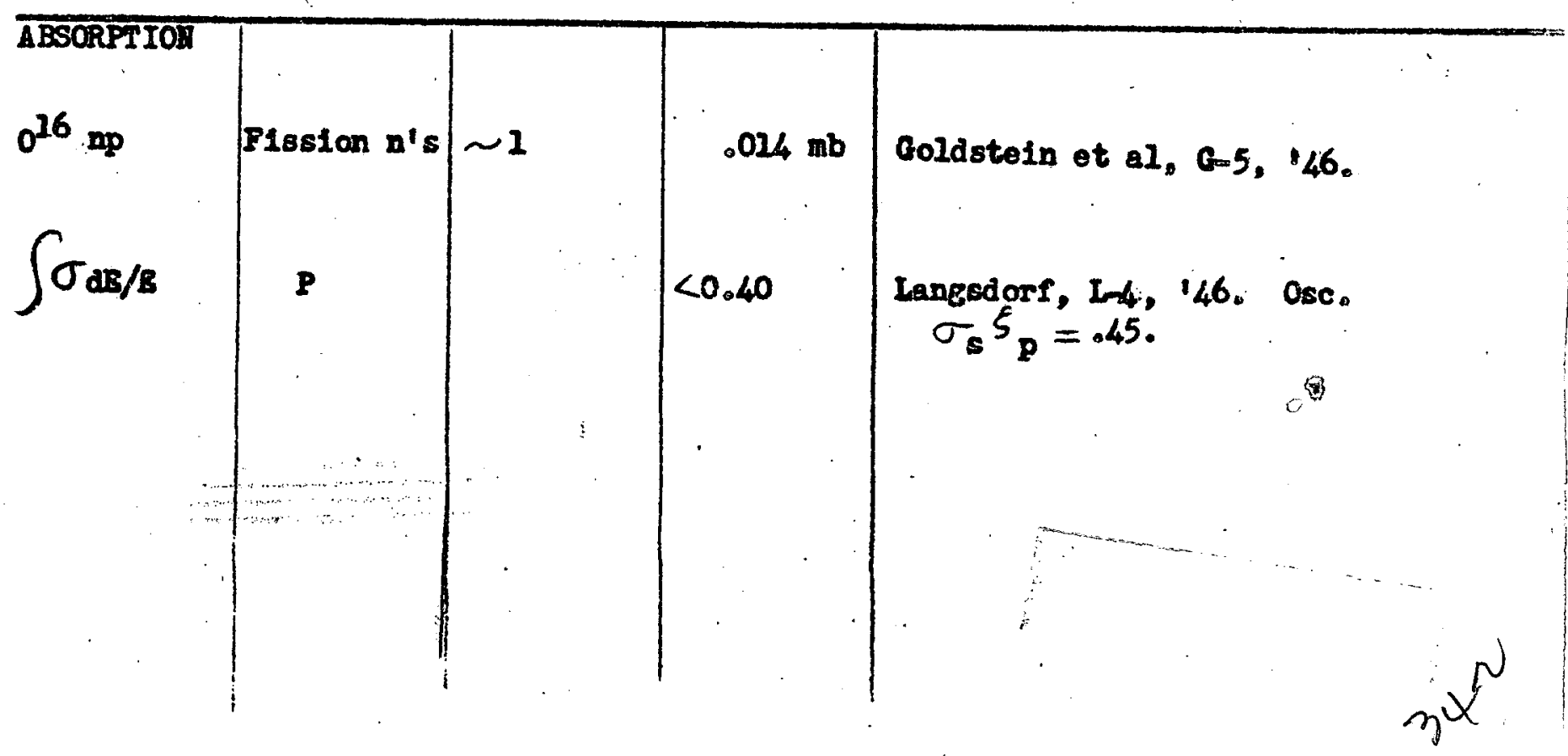




\begin{tabular}{|c|c|c|c|c|}
\hline & Sourree & $\begin{array}{l}\text { Neutron } \\
\text { Enerery }\end{array}$ & $\begin{array}{c}\sigma \\
\text { Lessured }\end{array}$ & \\
\hline TOIAL & $\begin{array}{l}D=D \\
C-D \\
R a T h-D \\
R a-B\end{array}$ & $\begin{array}{l}2.38-2.5 \\
0.18 \\
0.18 \\
0.09,0.4\end{array}$ & $\begin{array}{l}2.30 \\
2.7 \\
6.4 \\
6.3\end{array}$ & 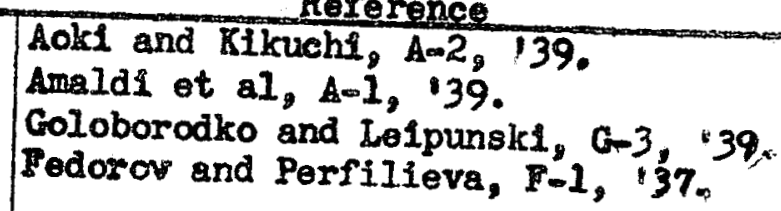 \\
\hline & $\begin{array}{l}\text { Photo } \mathbf{m}^{*} \\
\mathrm{D} \rightarrow \mathrm{D}\end{array}$ & $\begin{array}{l}.023-0.87 \\
2.1-2.8\end{array}$ & ${ }_{11}^{\text {graph WPB }}$ & $\begin{array}{l}\text { Wattenberg, } 7,8_{9}, 47 \text {. } \\
\text { Aoki, A- } 4,39 \text {. }\end{array}$ \\
\hline
\end{tabular}

SCATTERIMG

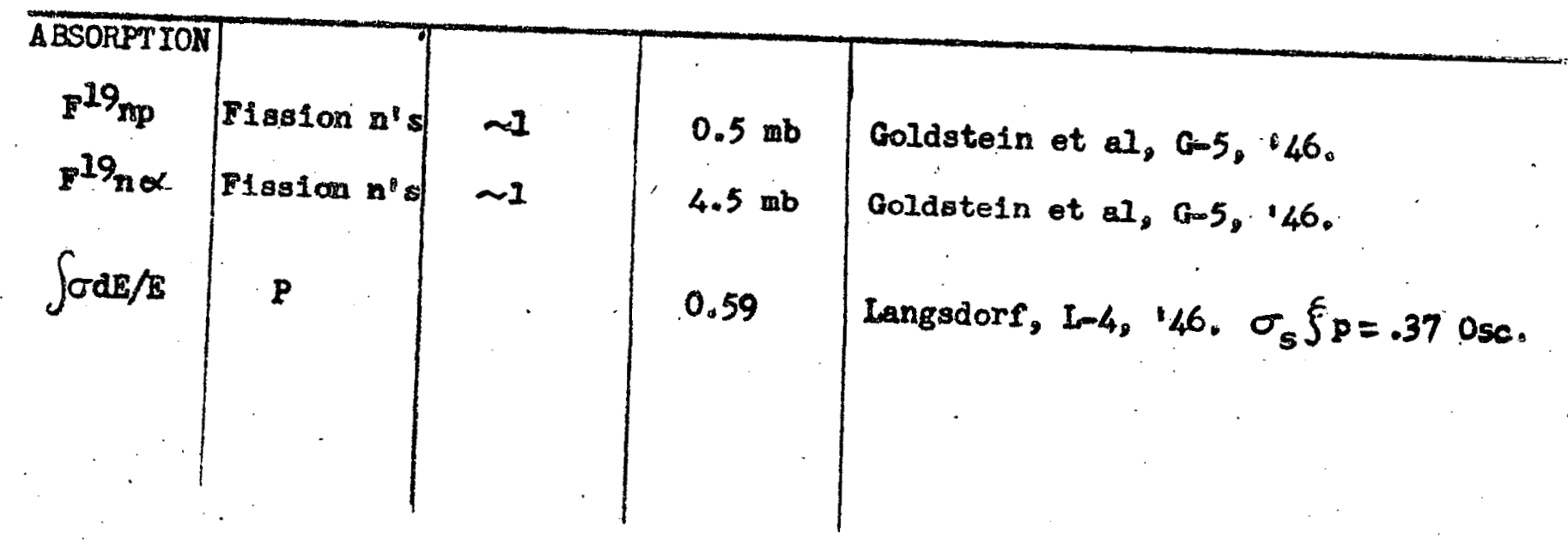



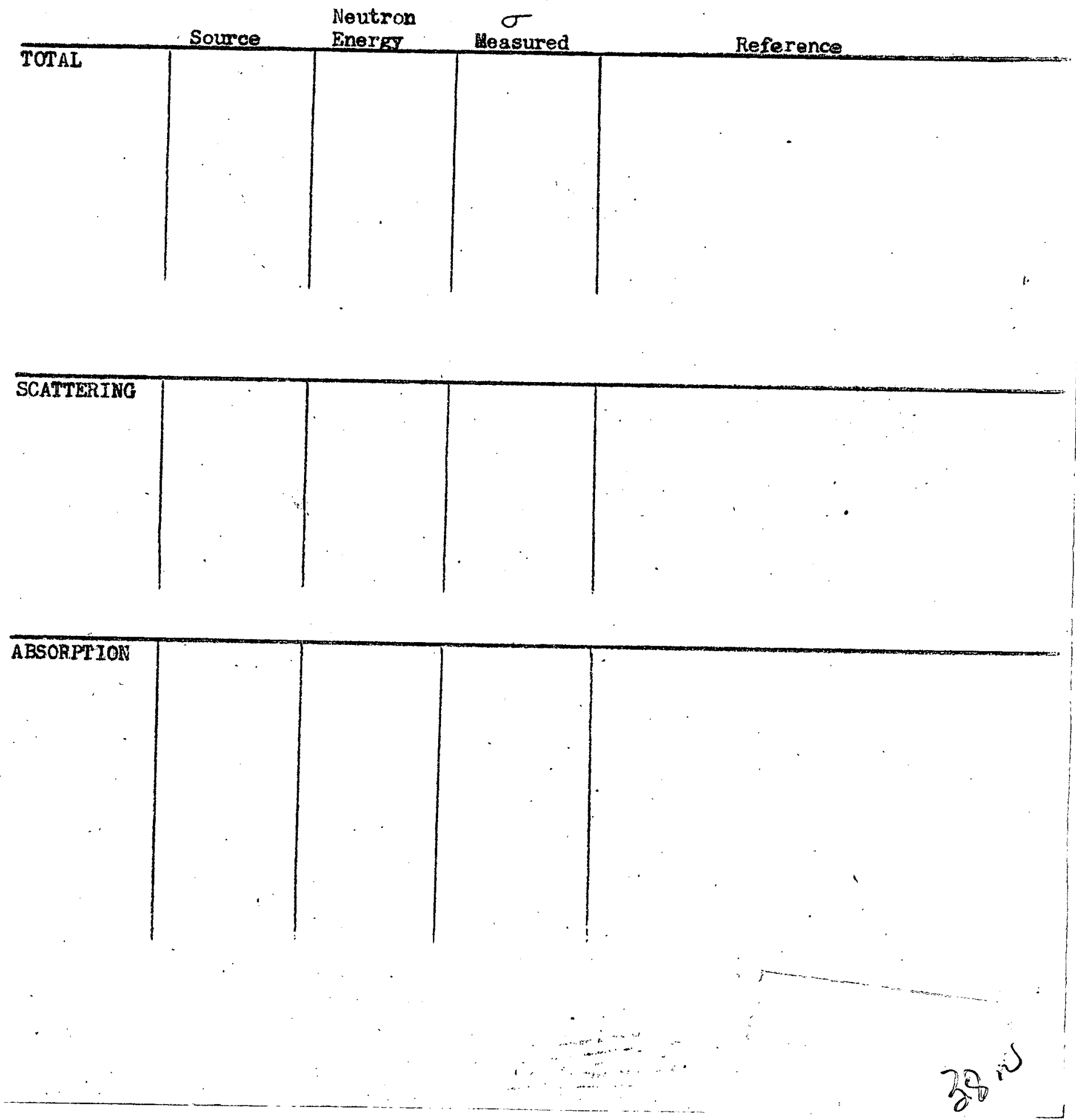
12. Sod1 um (Ha)
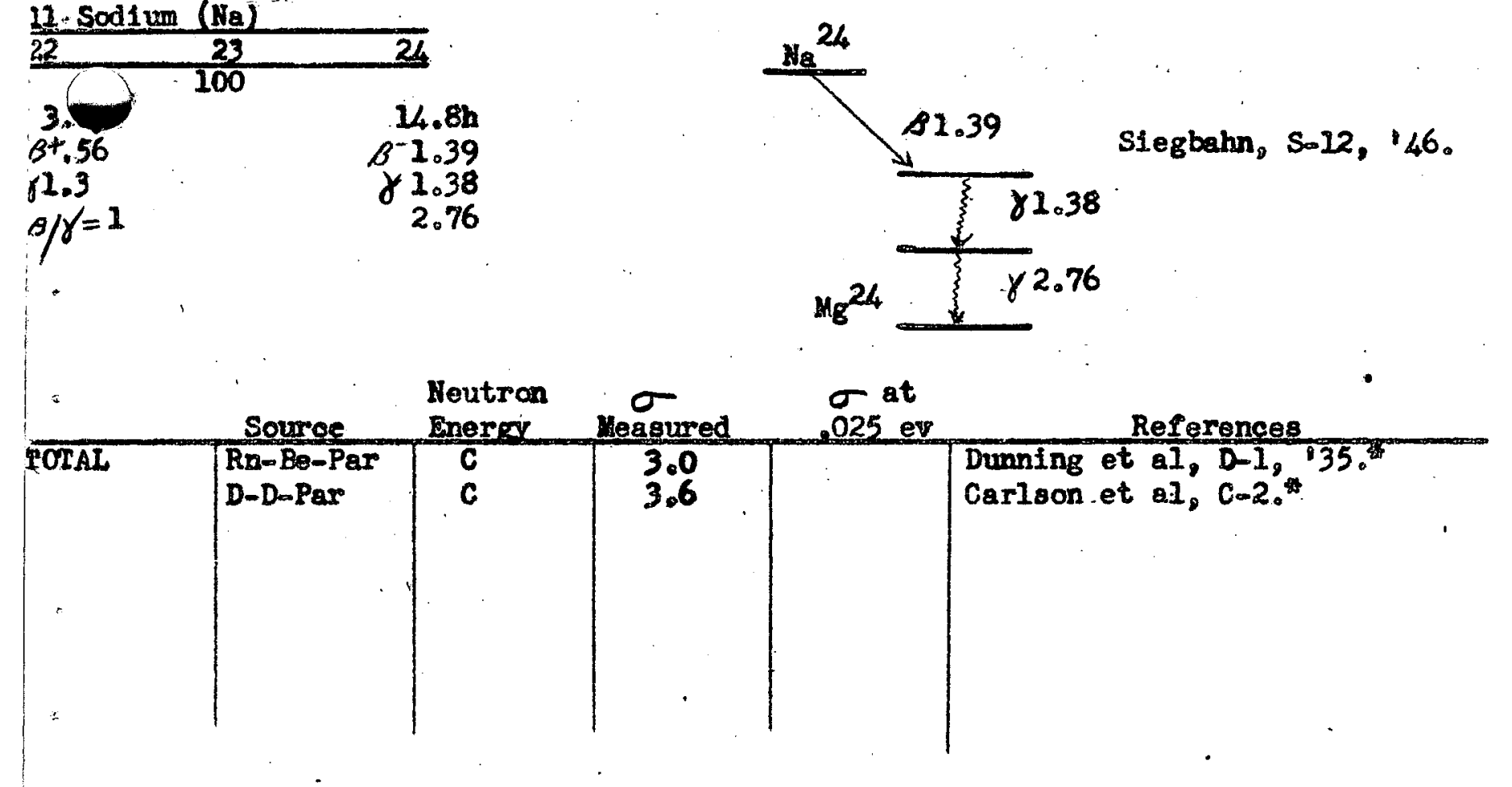

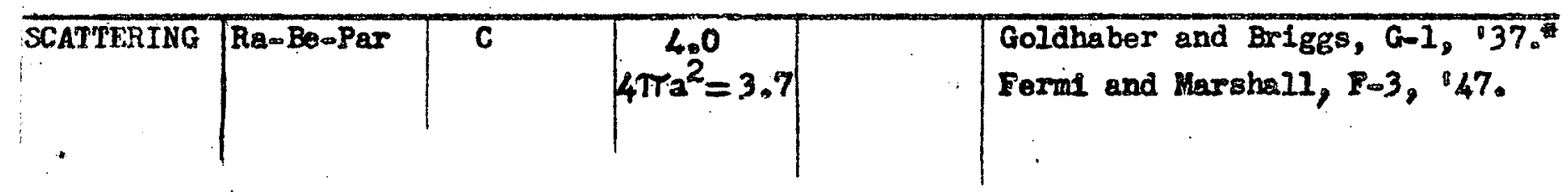

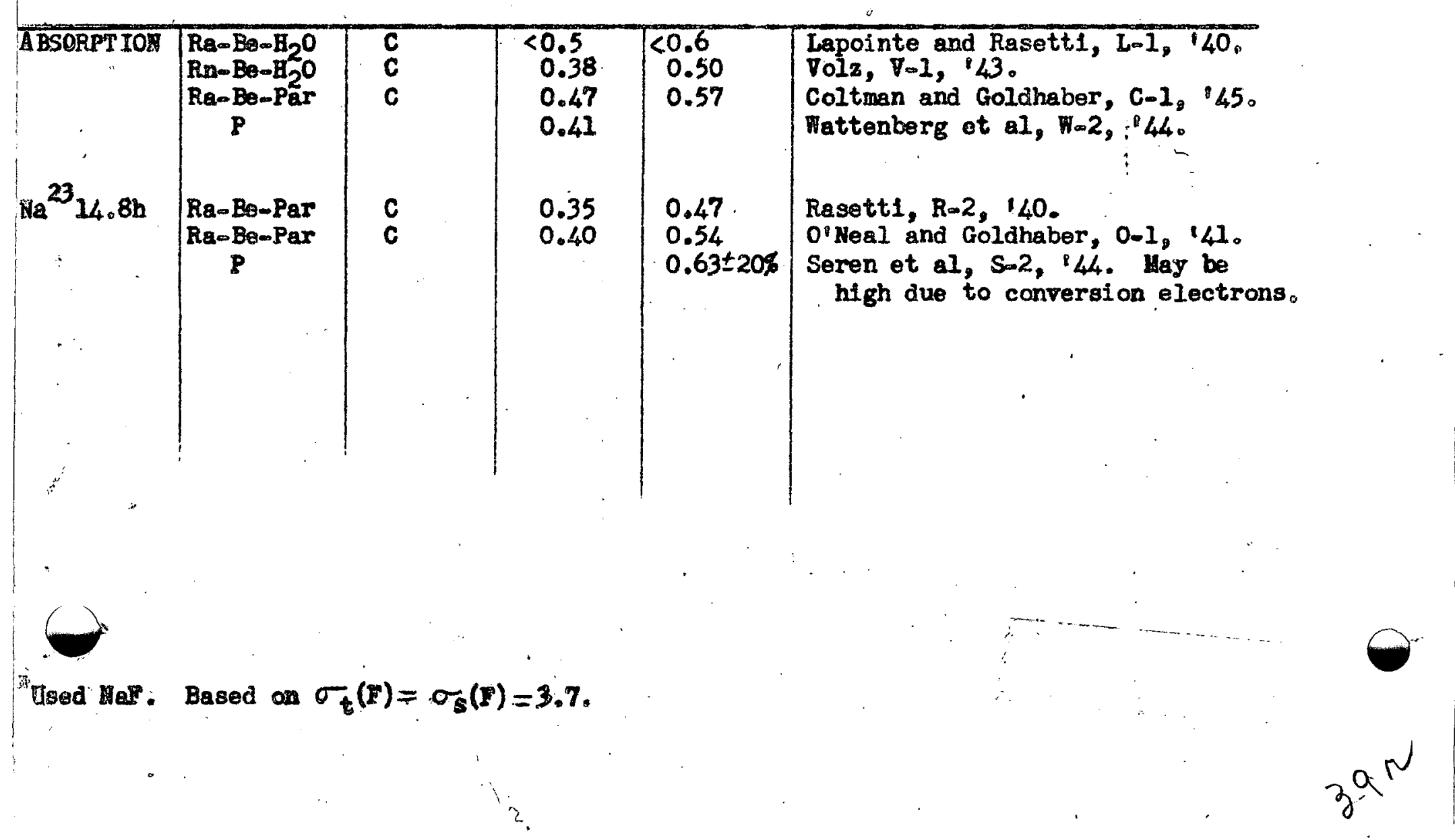




$$
\frac{E_{0}}{1710}
$$

\begin{tabular}{|c|c|c|c|c|}
\hline & Source & $\begin{array}{l}\text { Neutron } \\
\text { Energy }\end{array}$ & $\underset{\text { Heasured }}{\sigma}$ & References \\
\hline TOTAL & $\begin{array}{l}C-D \\
R d T h-D \\
R A-\gamma-B \theta \\
D=D\end{array}$ & $\begin{array}{l}0.18 \\
0.18 \\
0.09,0.4 \\
2.38-2.5 \\
2.3-2.8 \\
2.1-2.8 \\
2.5-2.9 \\
.023-0.88\end{array}$ & $\begin{array}{l}3.4 \\
3.2 \\
3.5 \\
3.33 \\
\text { graph } \\
\text { "1 } \\
\text { " }\end{array}$ & 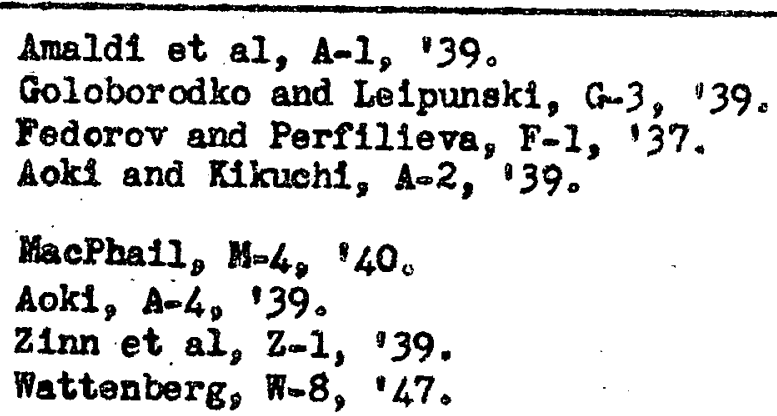 \\
\hline
\end{tabular}

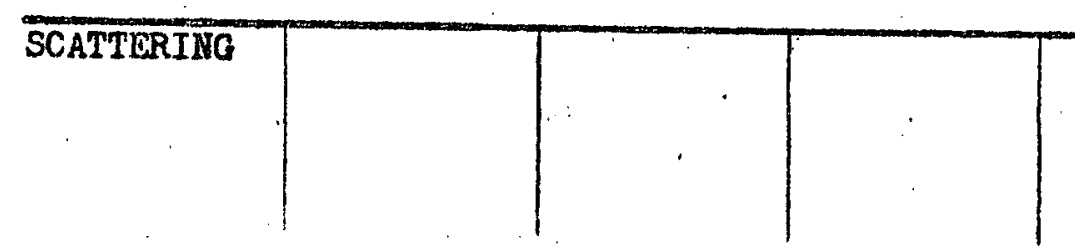

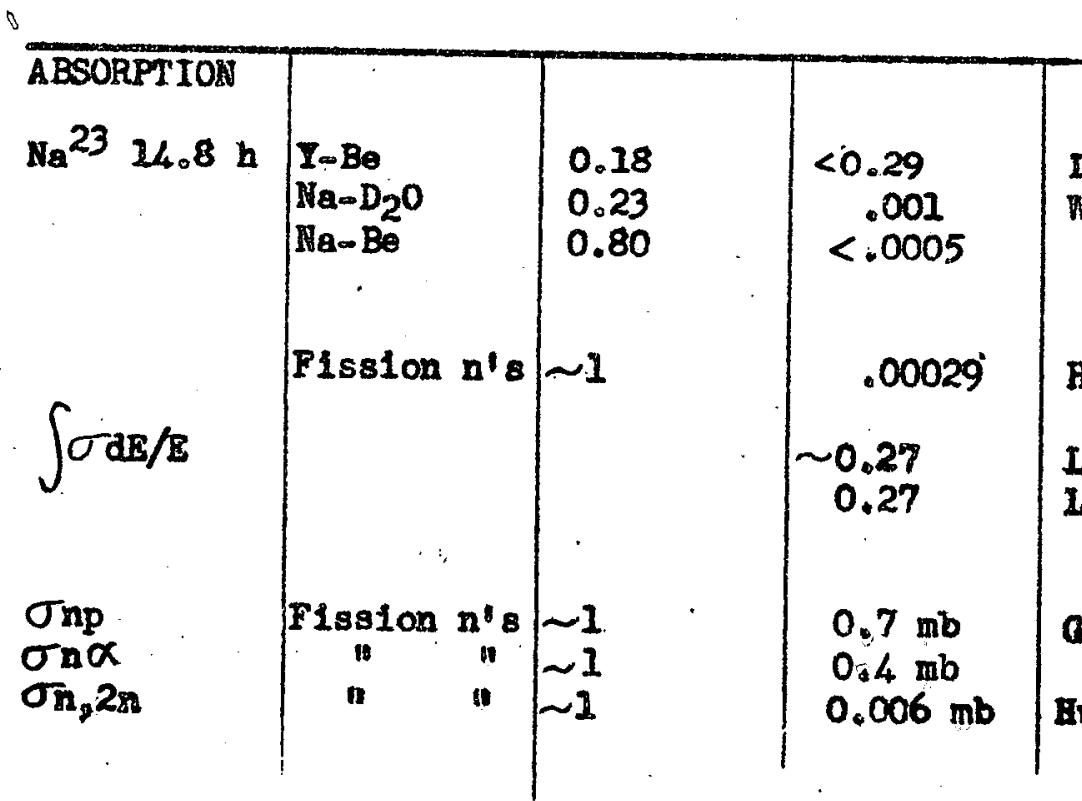

LA -140 )

Wattenberg, $1-6,7 / 4$. Based on gold crose sections of :41 and .13 for $.23 \mathrm{mov}$ and $.80 \mathrm{mev}$ respectively.

Hughes et al, H-2, $: 46$.

Langsdorf et $a I_{,} I-4,747$. Os0. $\sigma_{8} \xi_{p}=27$ Lichtenberger ot al, $\operatorname{Lin} 8,47$. B abs. .051 due to resonance.

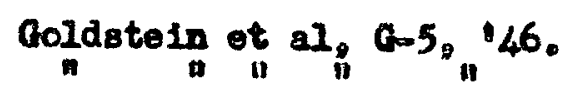

Eughes ot a1, H:8, 46. 
TSOW

12 Mignesium (us)
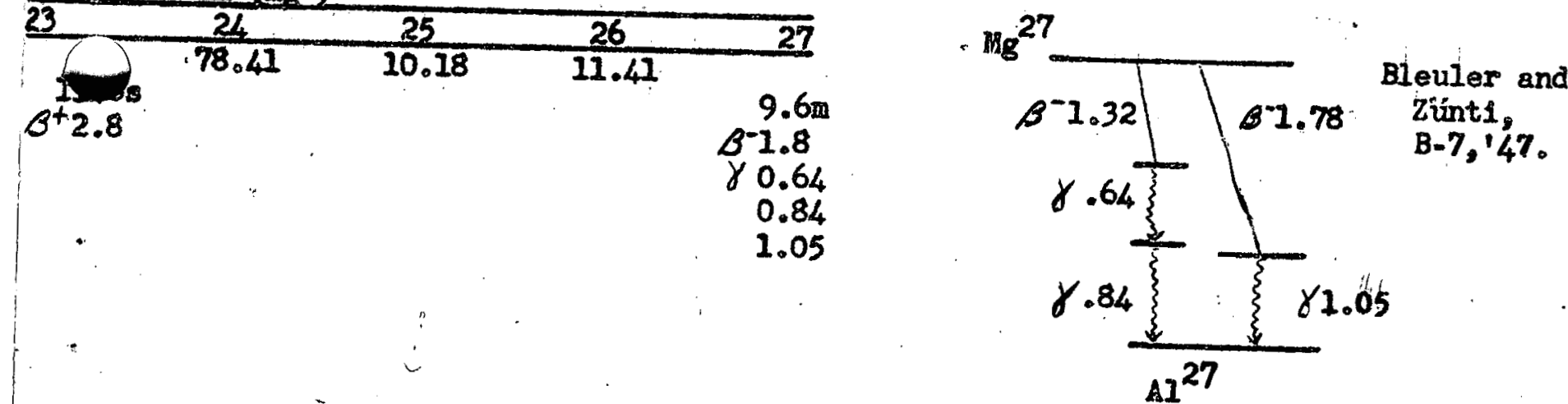

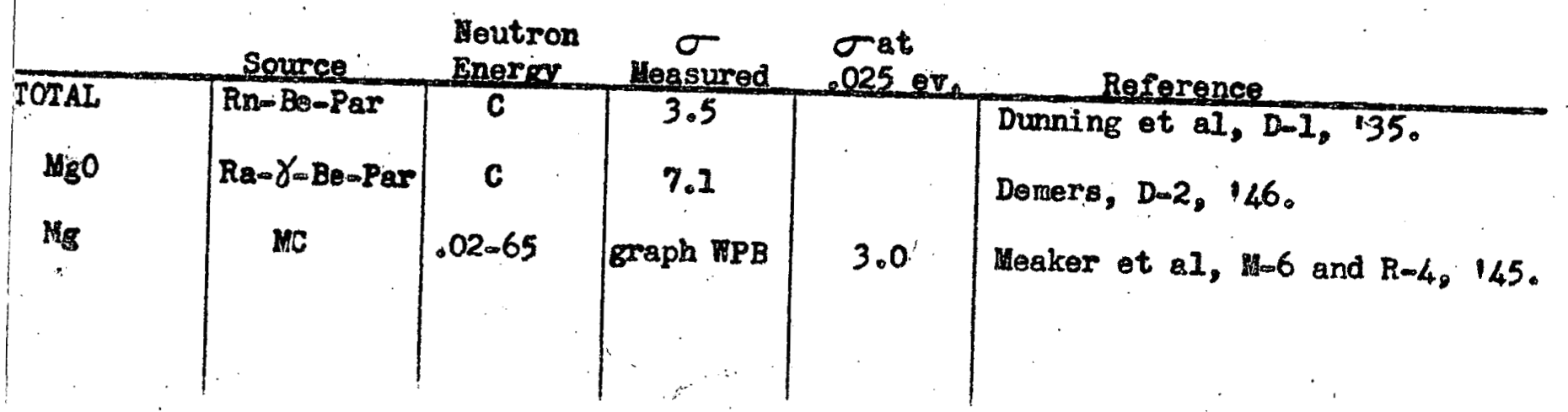

\begin{tabular}{|c|c|c|c|c|}
\hline SCATTERING & $\begin{array}{c}\text { Ra-Be-Par } \\
\text { Ram Bs-Par } \\
\mathbf{P}\end{array}$ & $\underset{\text { Ep } 1-C d}{c}$ & $\begin{array}{c}3.0 \\
4.2 \\
3.6 \\
4 \pi_{8}{ }^{2}=3.7\end{array}$ & 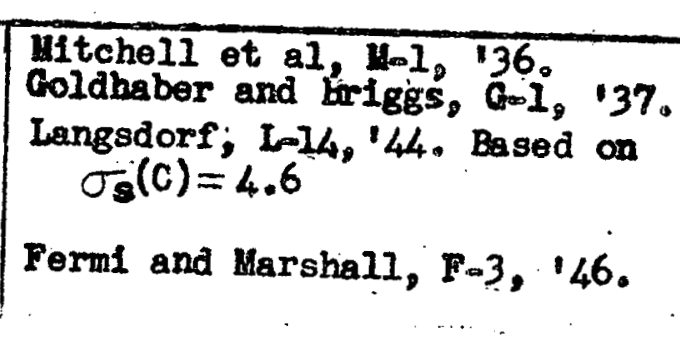 \\
\hline
\end{tabular}

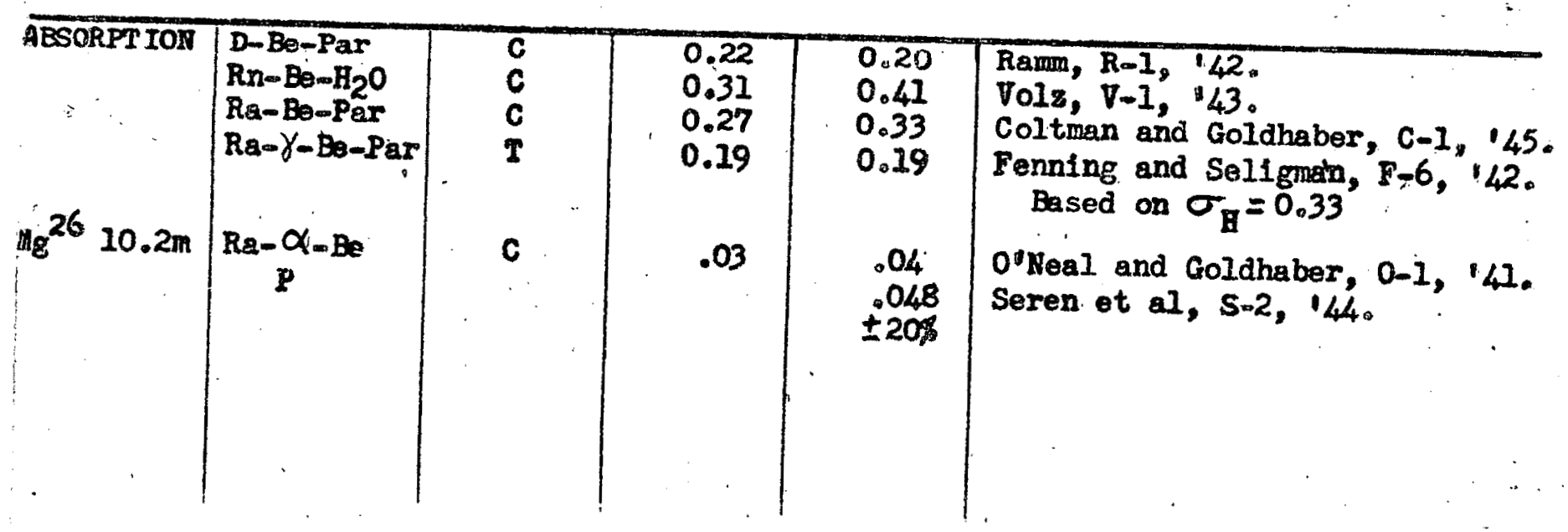


Nagnesfun






\section{AIuminum (AI)}

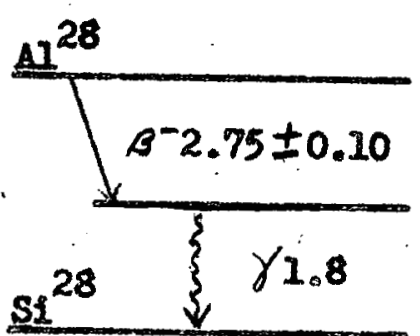

Blevier and Zuint1, $B-7,47$.

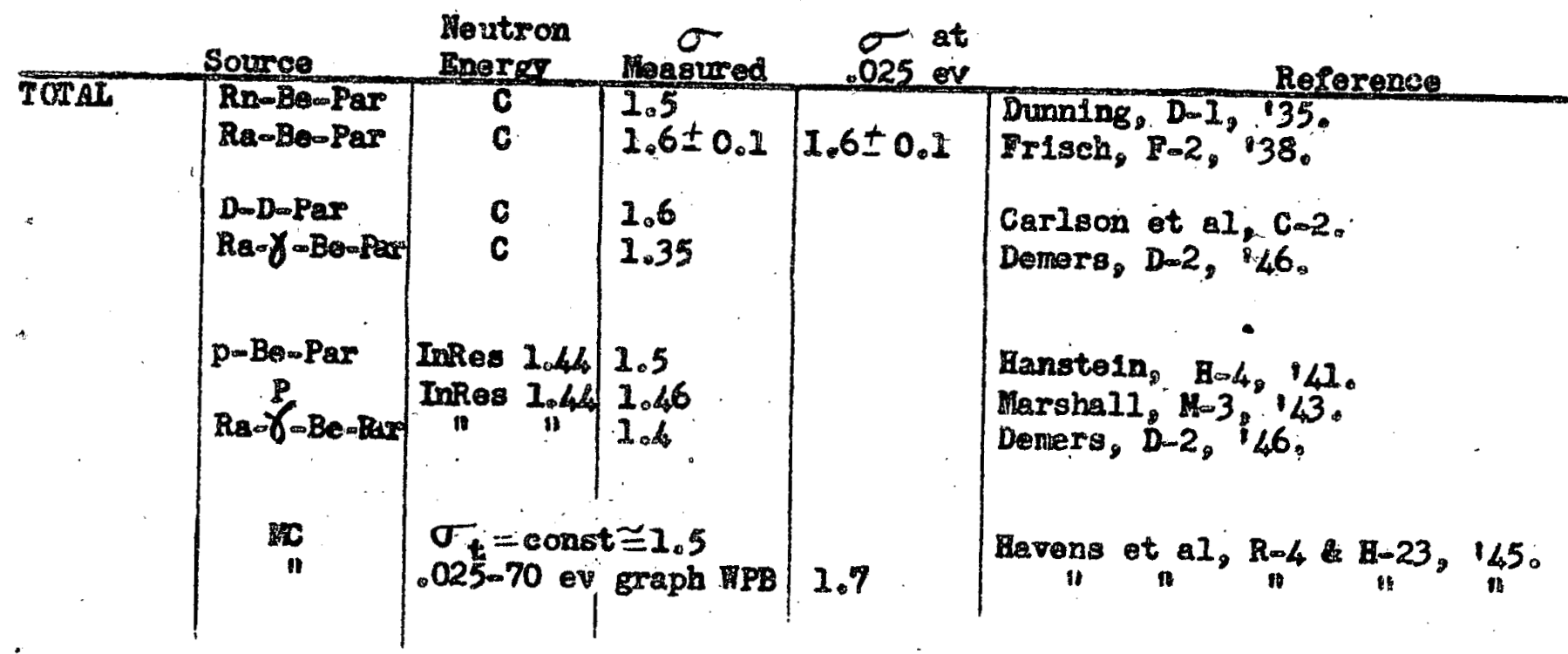

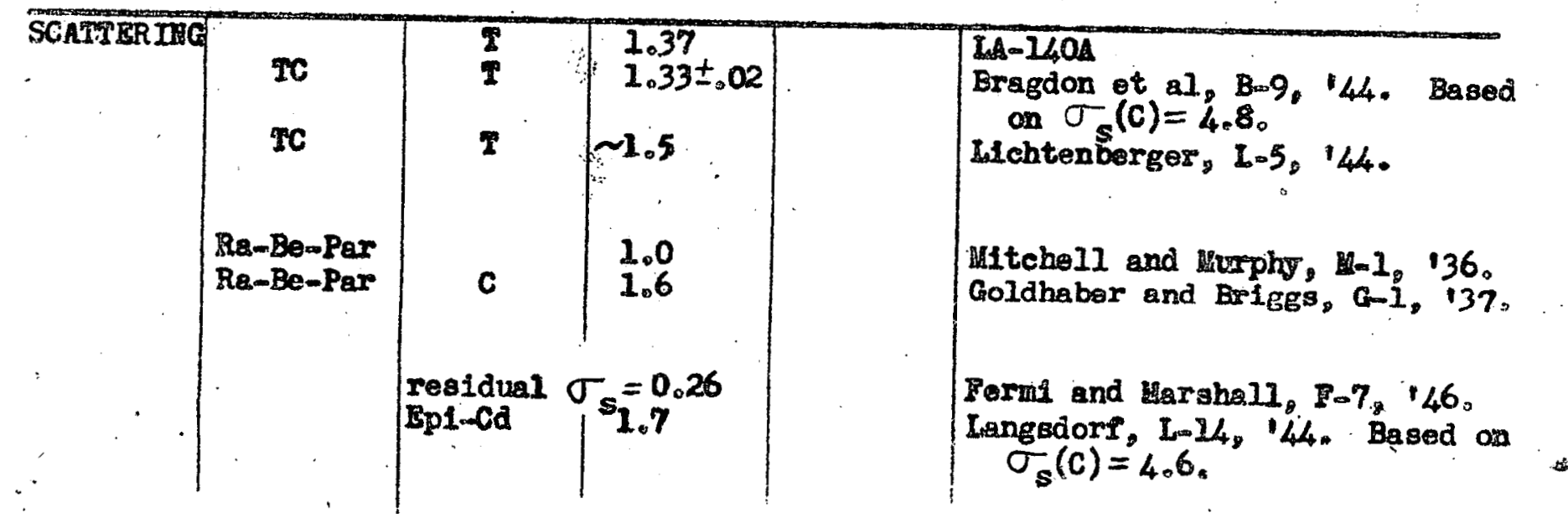

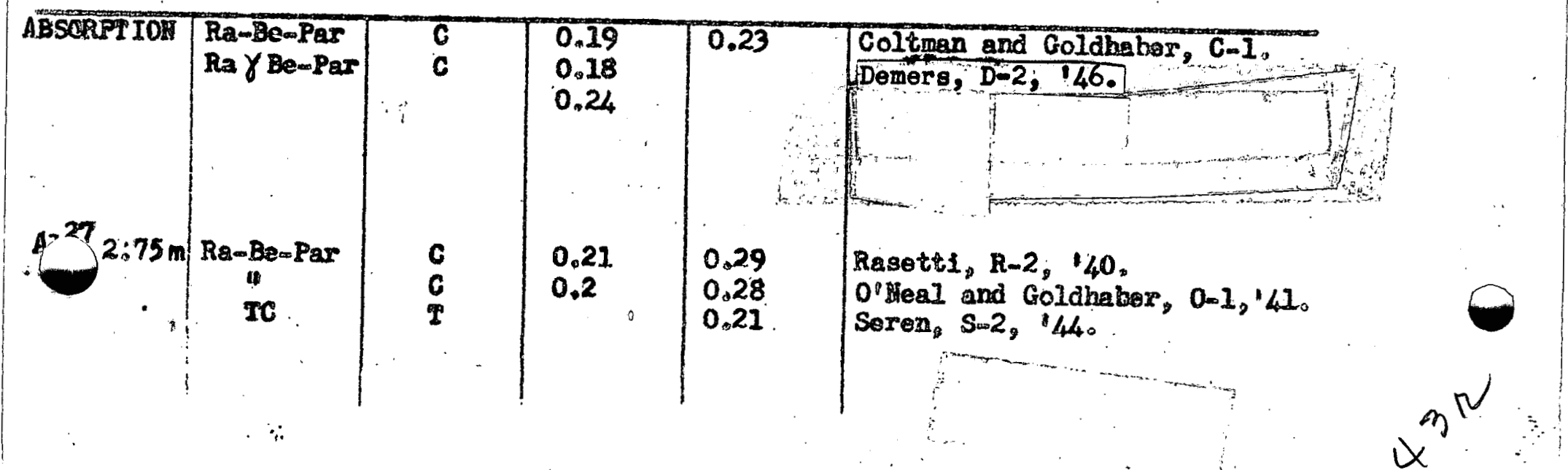




\section{TAST}

Aluainum (continued)

(AI)

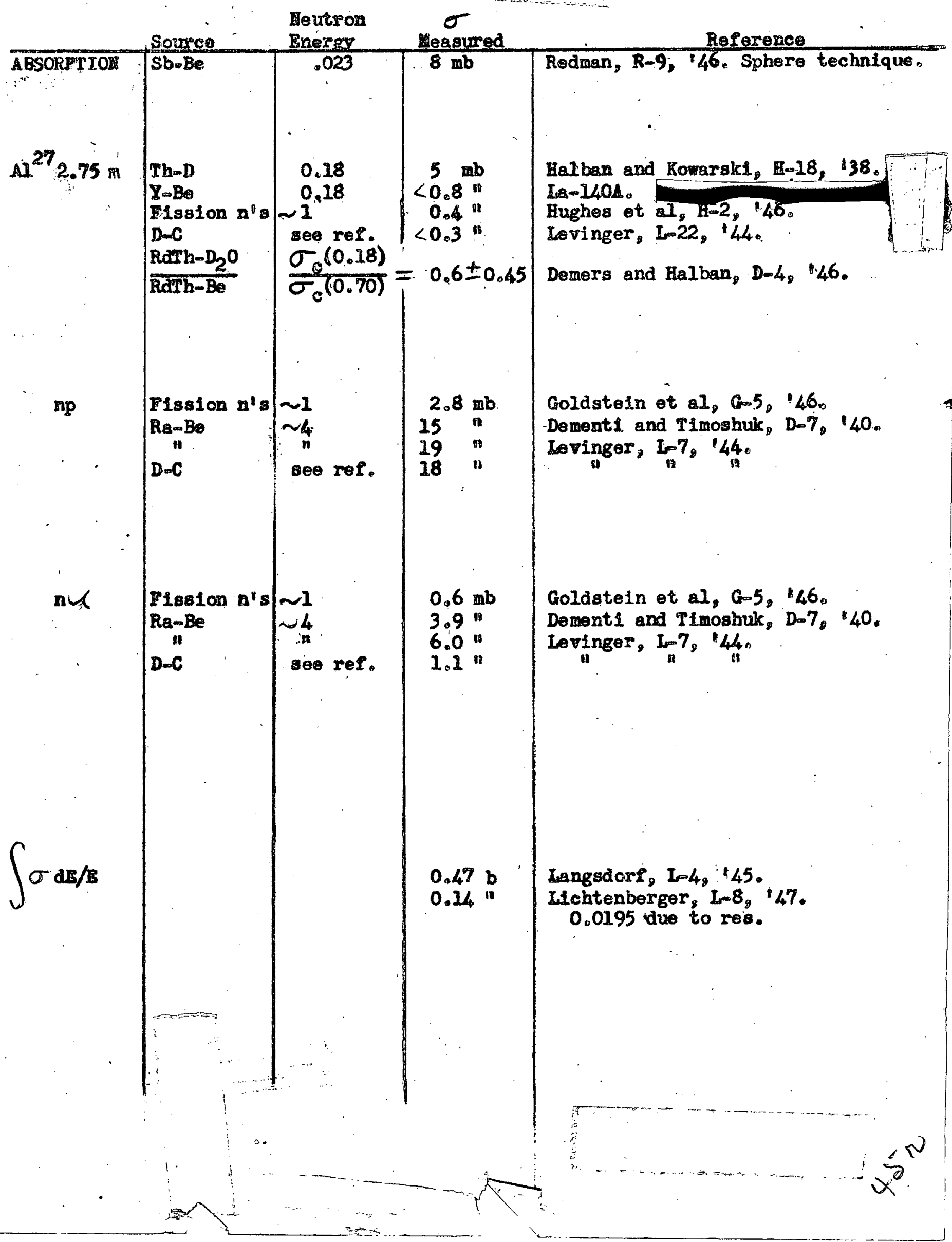




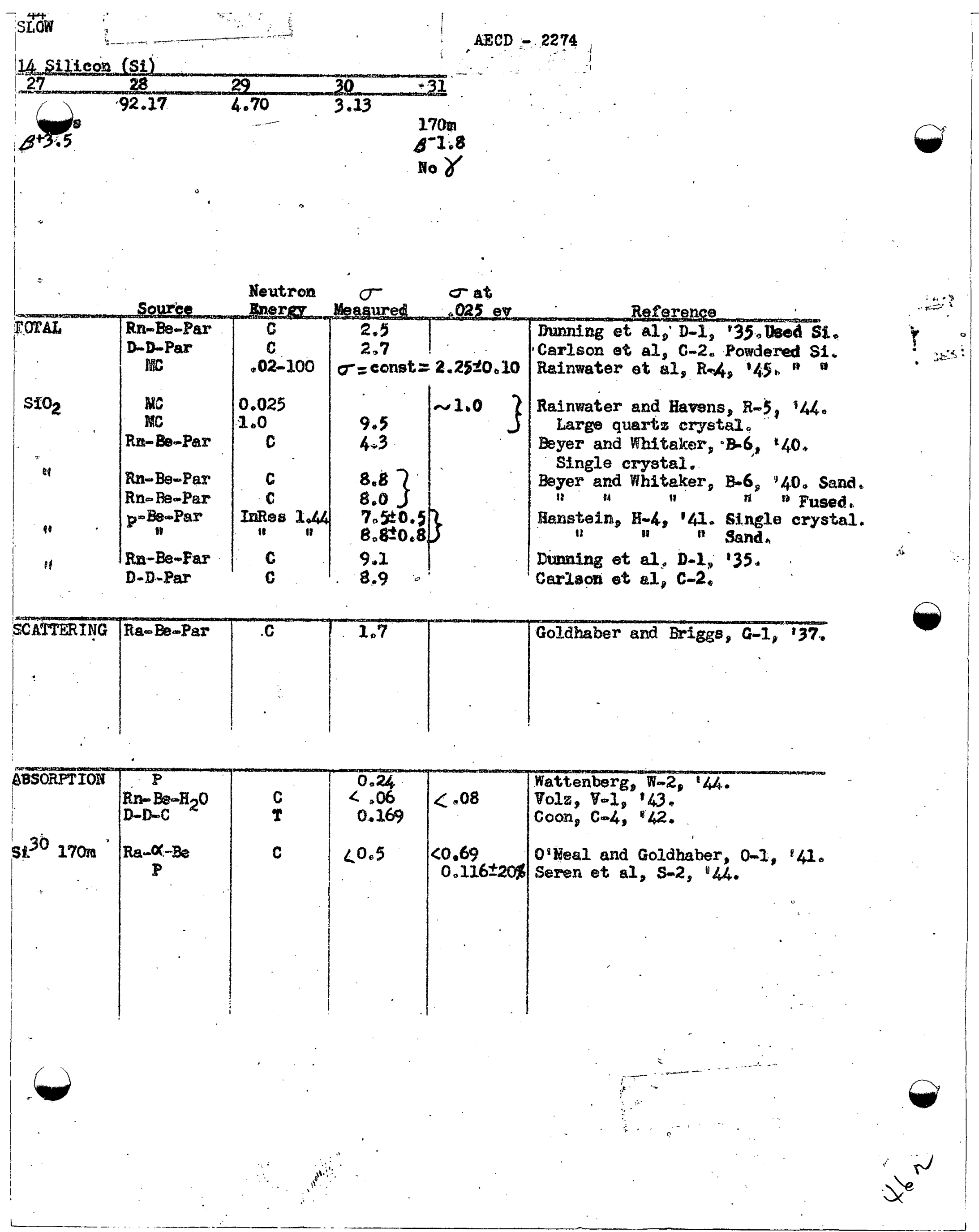




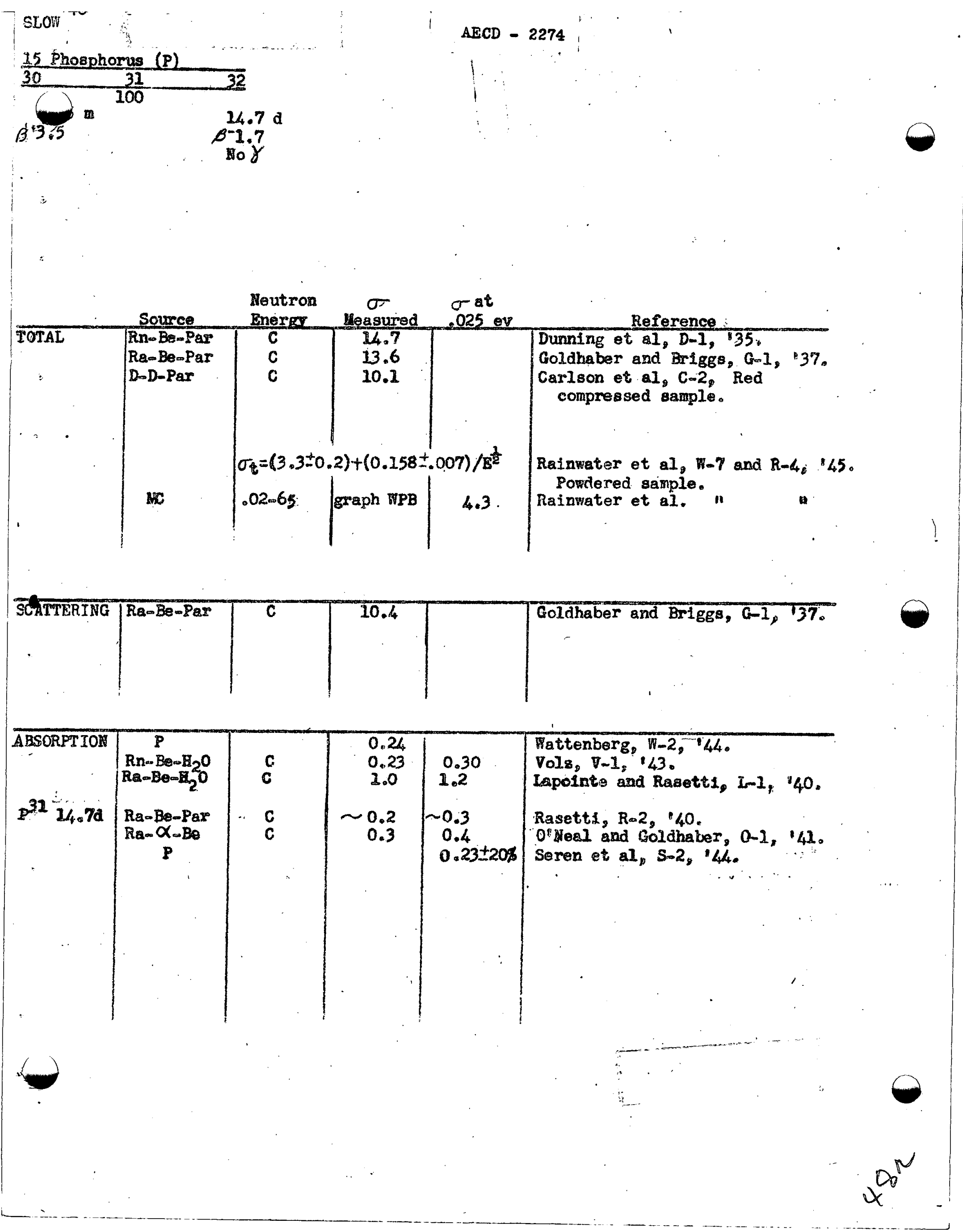




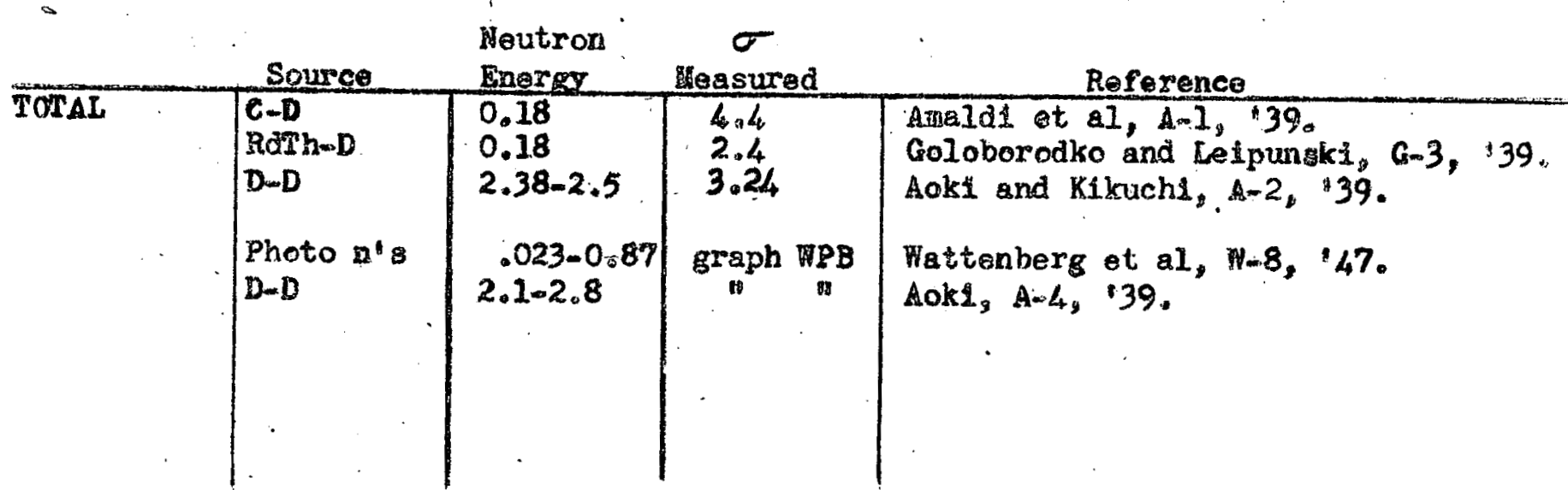

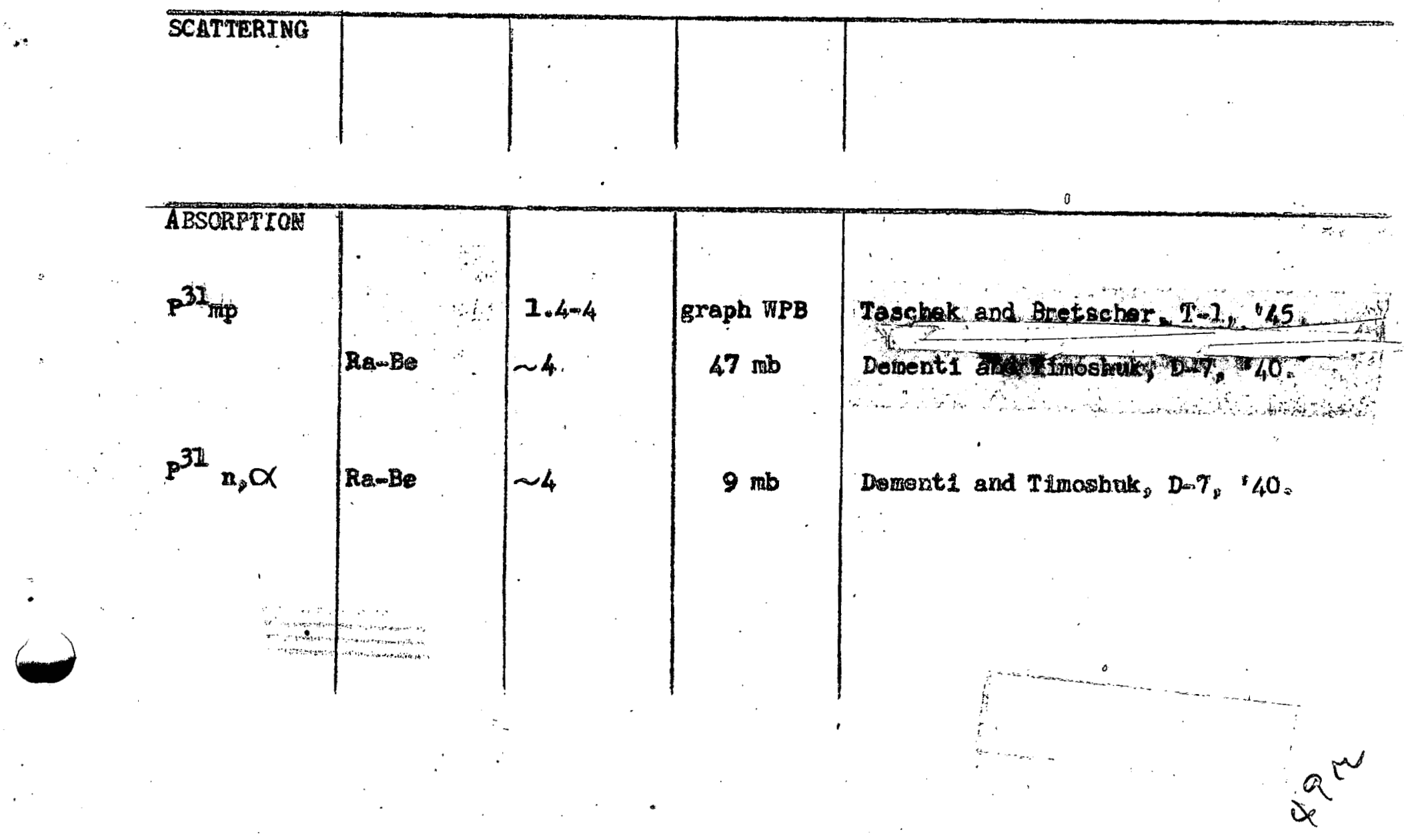




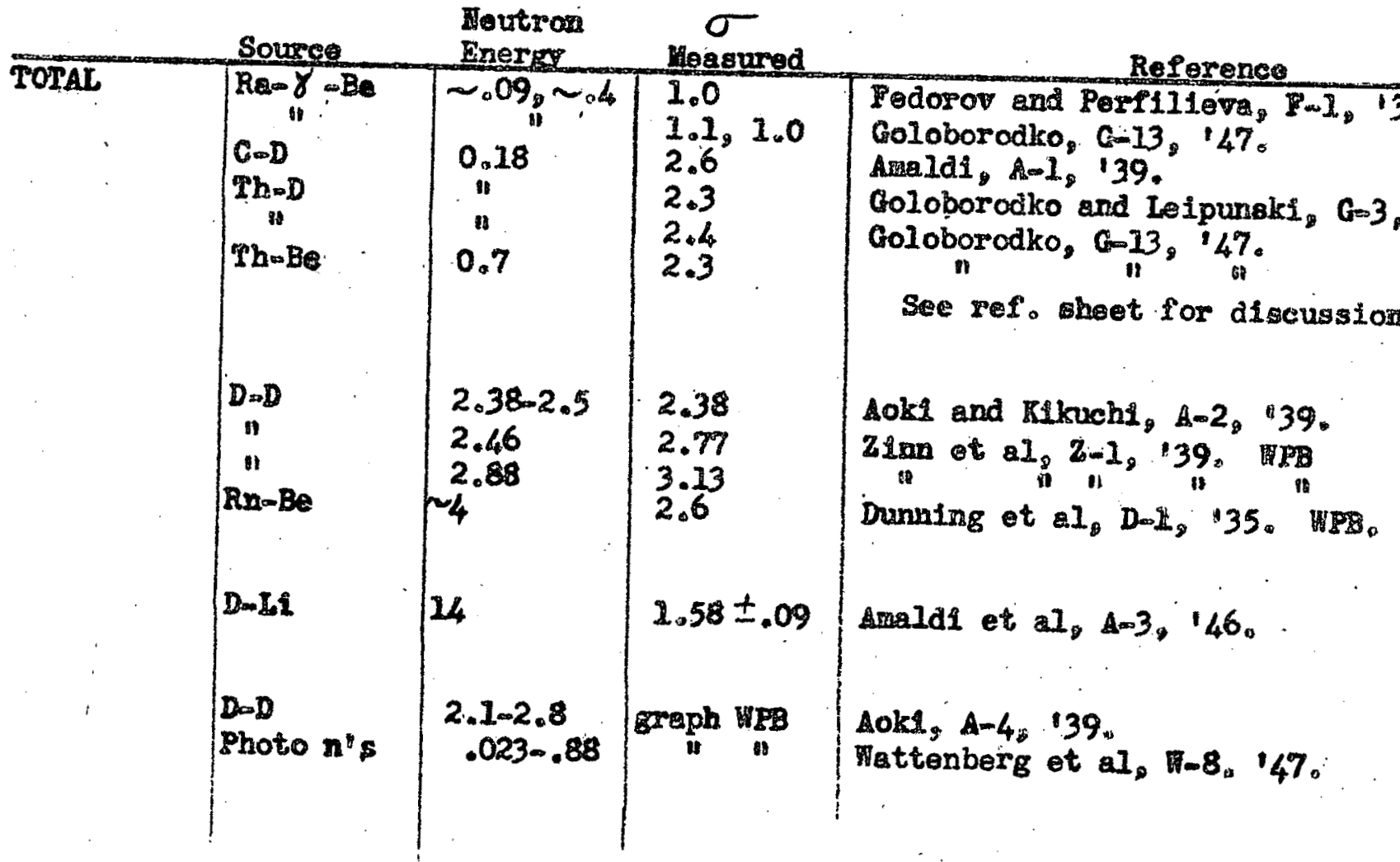

ScaTTERIKa

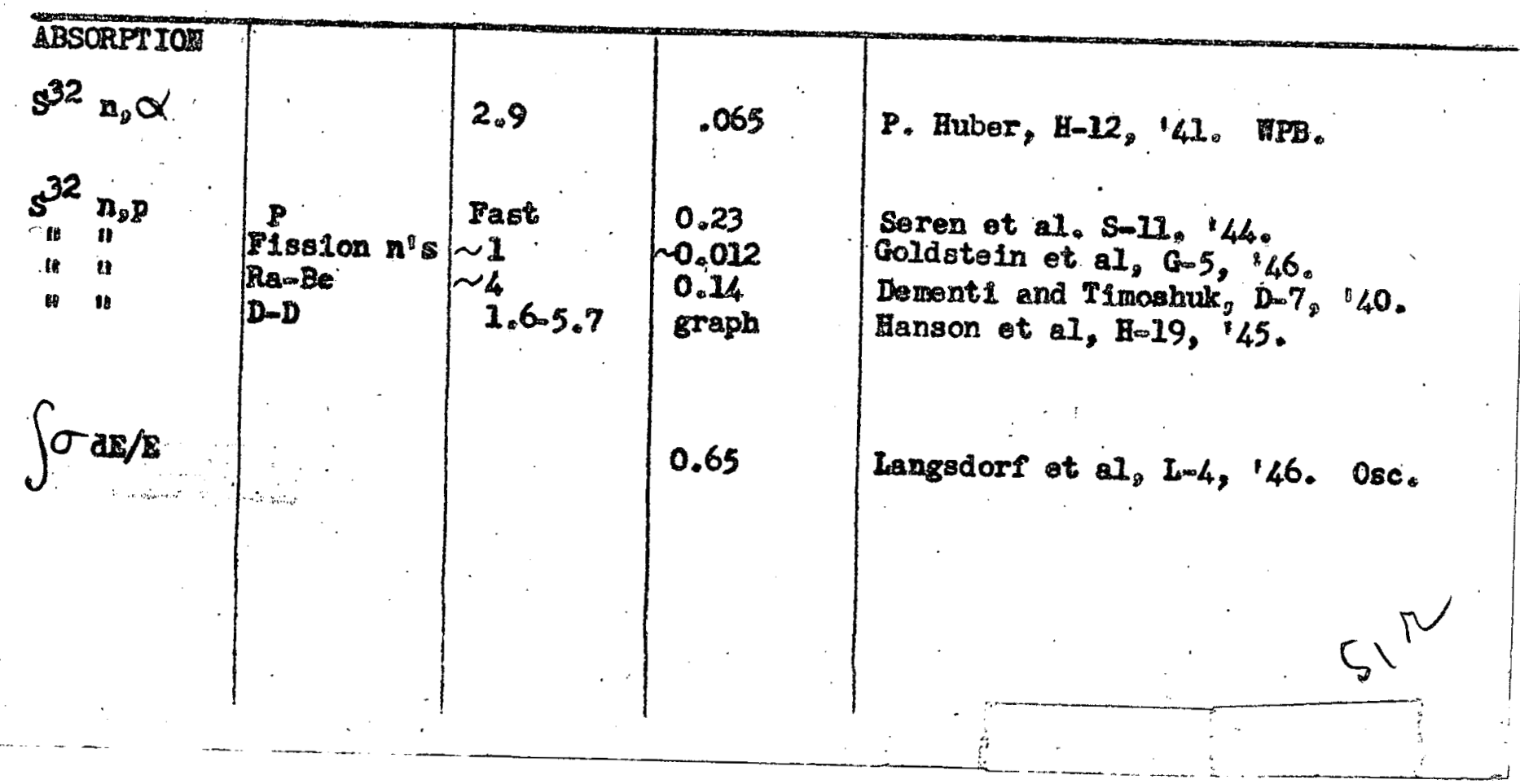


Chlorine

\section{Regonances}

$\frac{B_{0}}{9.9}$

9.9
4.4 possible levels

$c^{37}--1800$

\begin{tabular}{|c|c|c|c|c|}
\hline \multirow[t]{4}{*}{ TOTAT } & $\frac{\text { Sourge }}{\text { Rem } / \mathrm{Be}}$ & $\begin{array}{l}\text { Neutros } \\
\text { Enerpes } \\
\end{array}$ & $\frac{\text { Hegsured }}{2}$ & Reference \\
\hline & 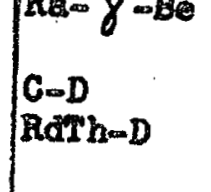 & $\begin{array}{l}\sim .09, \sim 0.4 \\
0.18 \\
0.18\end{array}$ & $\begin{array}{l}3.5 \\
2.7 \\
3.8\end{array}$ & $\begin{array}{l}\text { Fedoror and Perfilleva, }, 1,137 . \\
\text { Amaldi et al, A-1, } 39 . \\
\text { Coloborodko and Leipunski, } 6-3,139 .\end{array}$ \\
\hline & $\begin{array}{l}D-D \\
D-D\end{array}$ & $\begin{array}{l}2.38-2.5 \\
2.88 \pm .04\end{array}$ & $\begin{array}{l}2.78 \\
3.4\end{array}$ & $\begin{array}{l}\text { Aoki, A-2, } 39 . \\
\text { Zimn et } 81, Z=1, \quad 39 .\end{array}$ \\
\hline & $D=L 1$ & 25.4 & 1.88 & Shorr, S-29, $: 45$ \\
\hline
\end{tabular}

\section{SCATTER ING

$\mid$

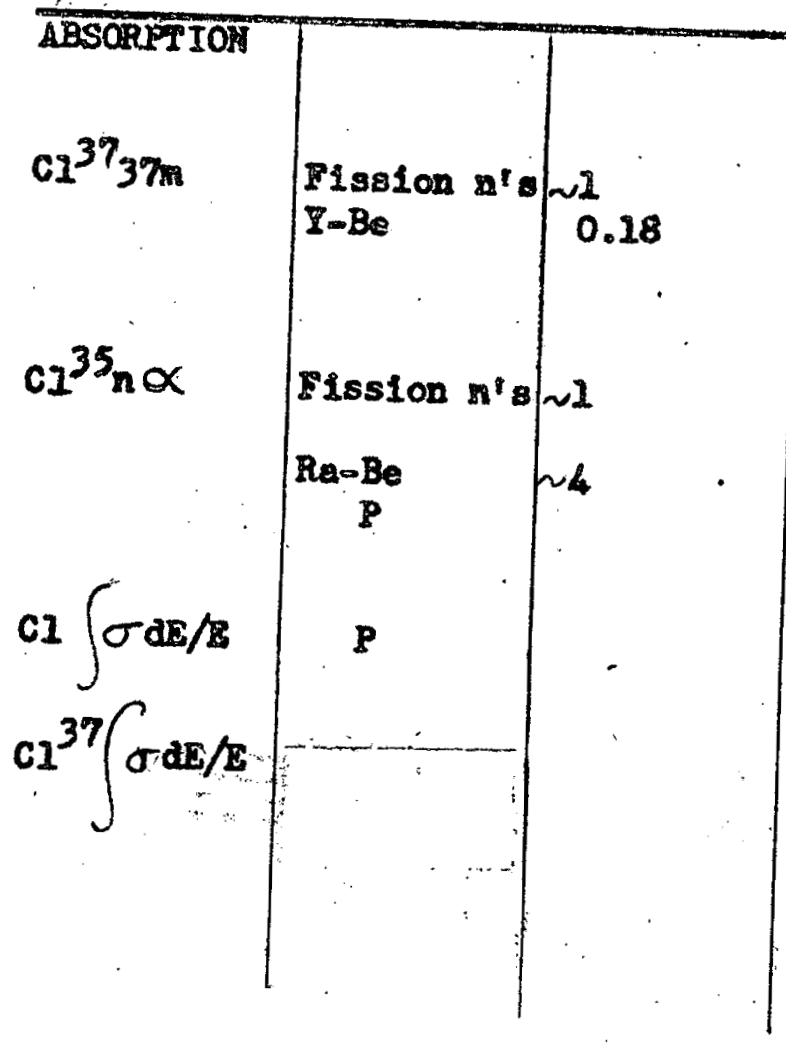

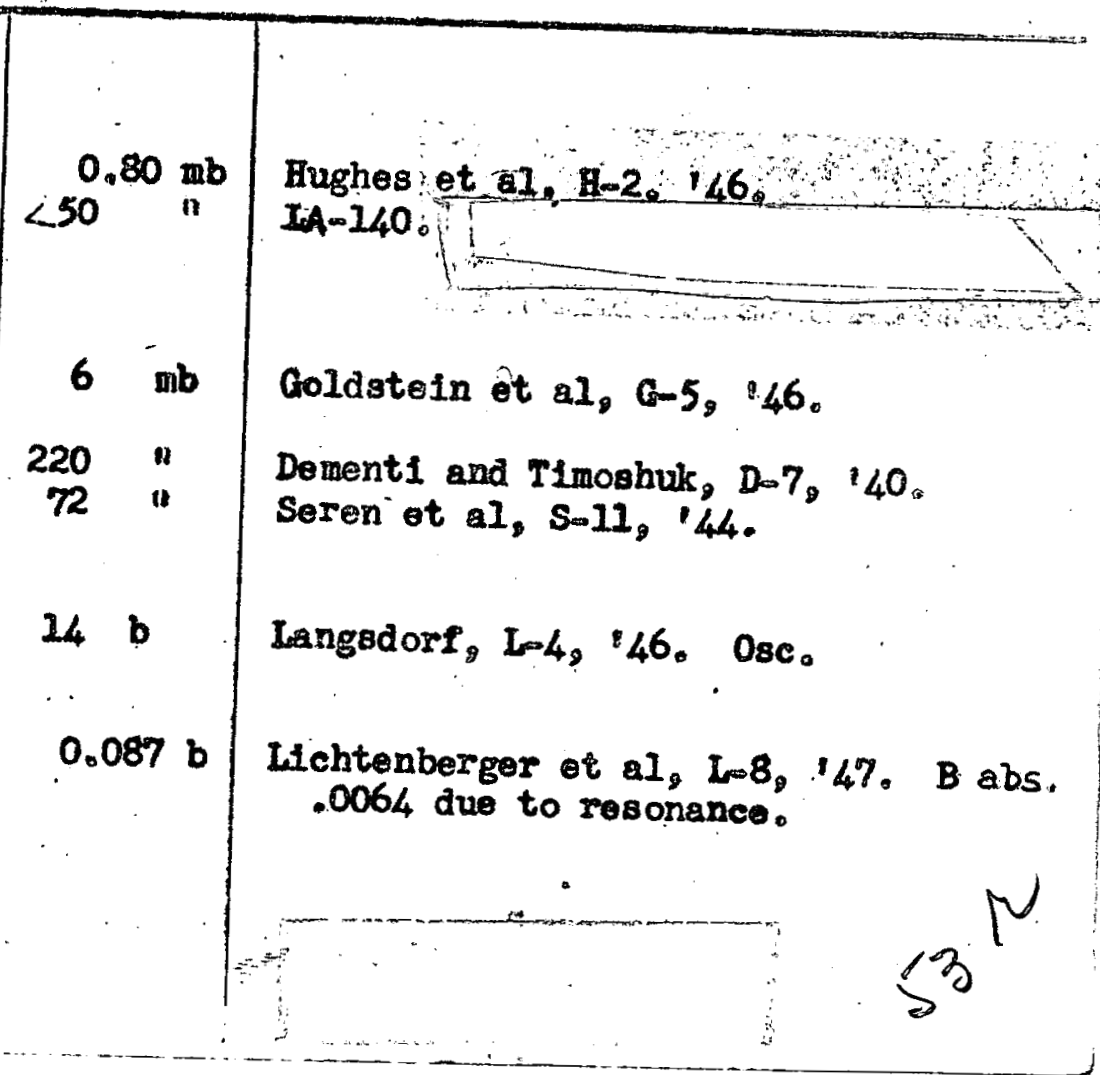




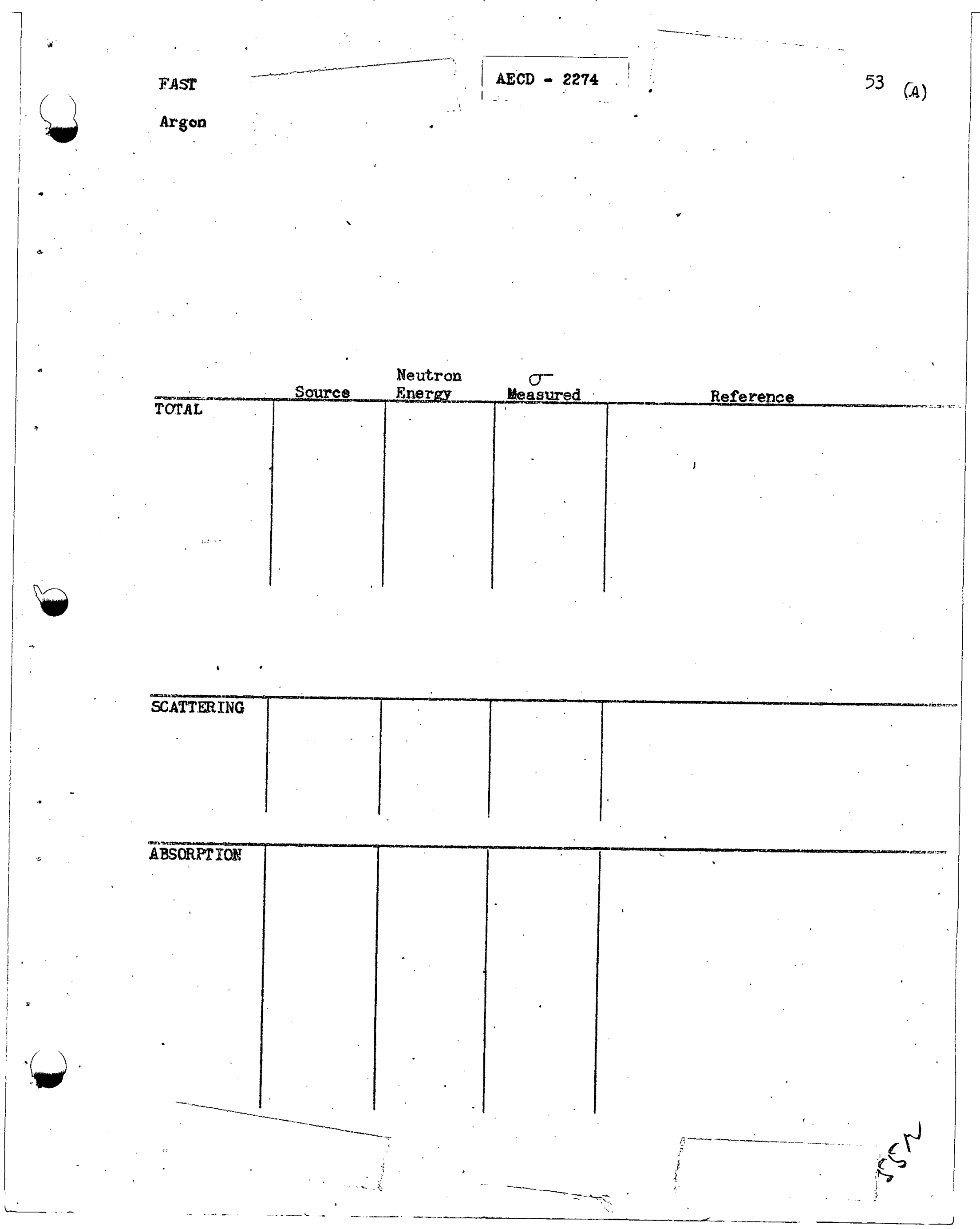




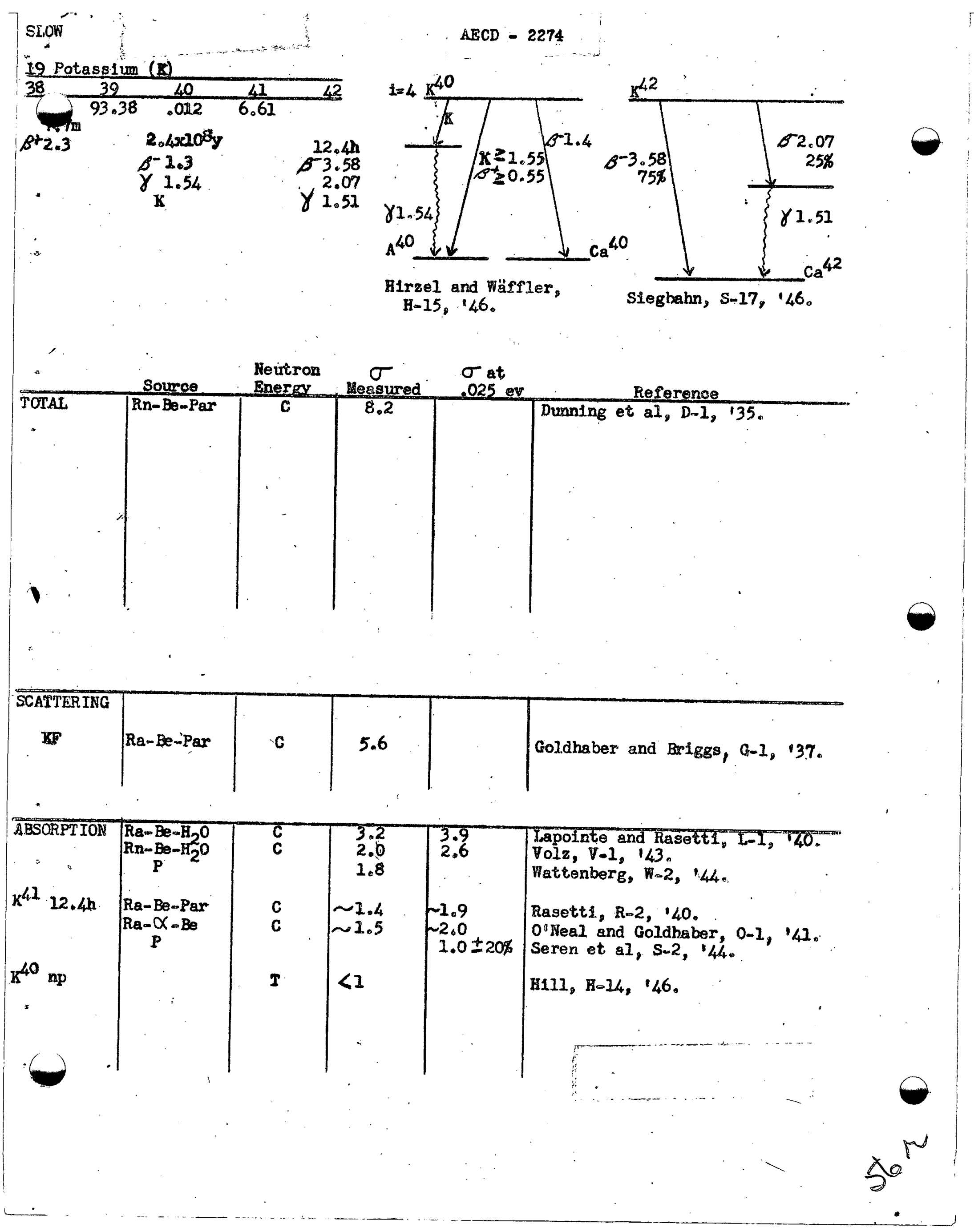




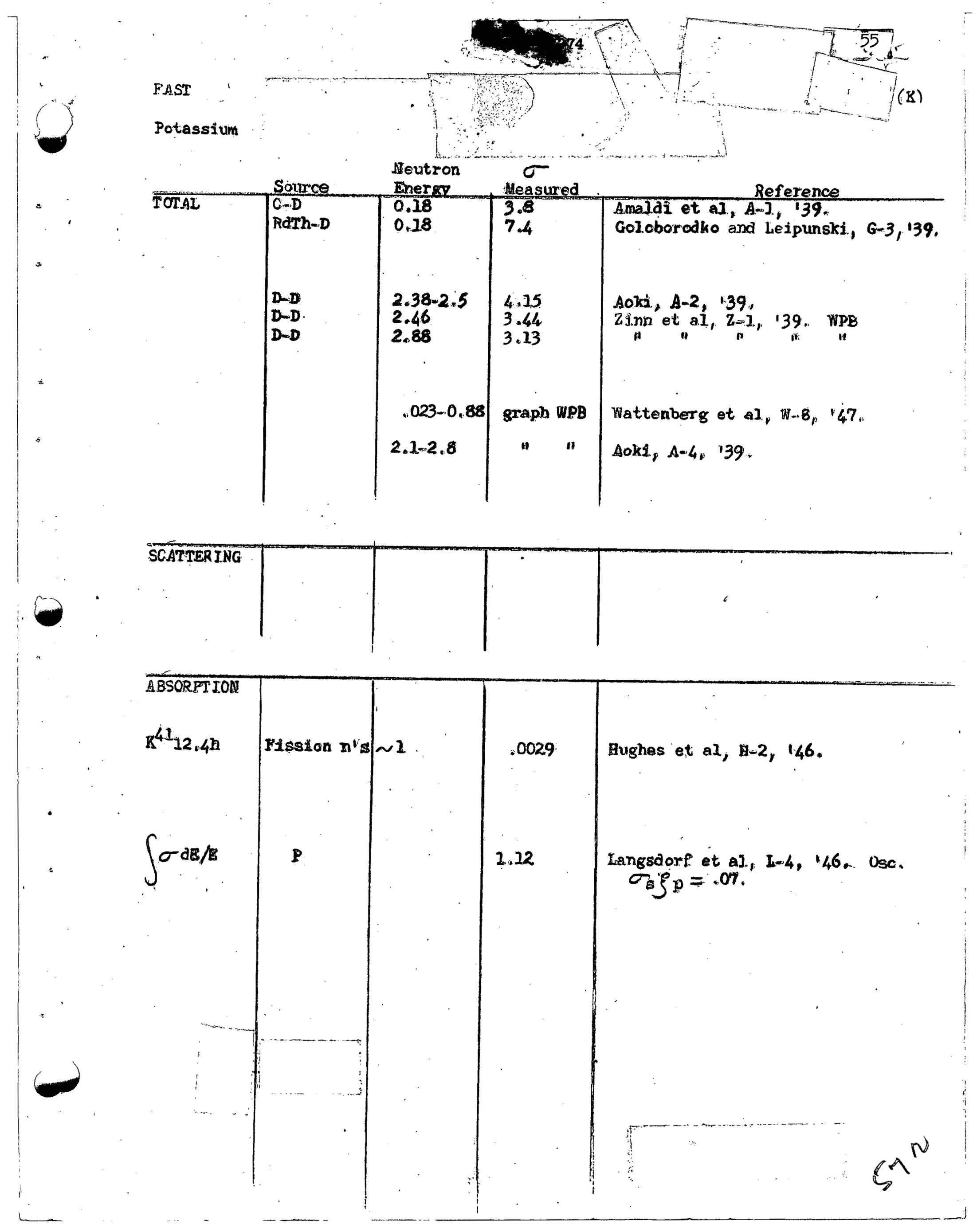




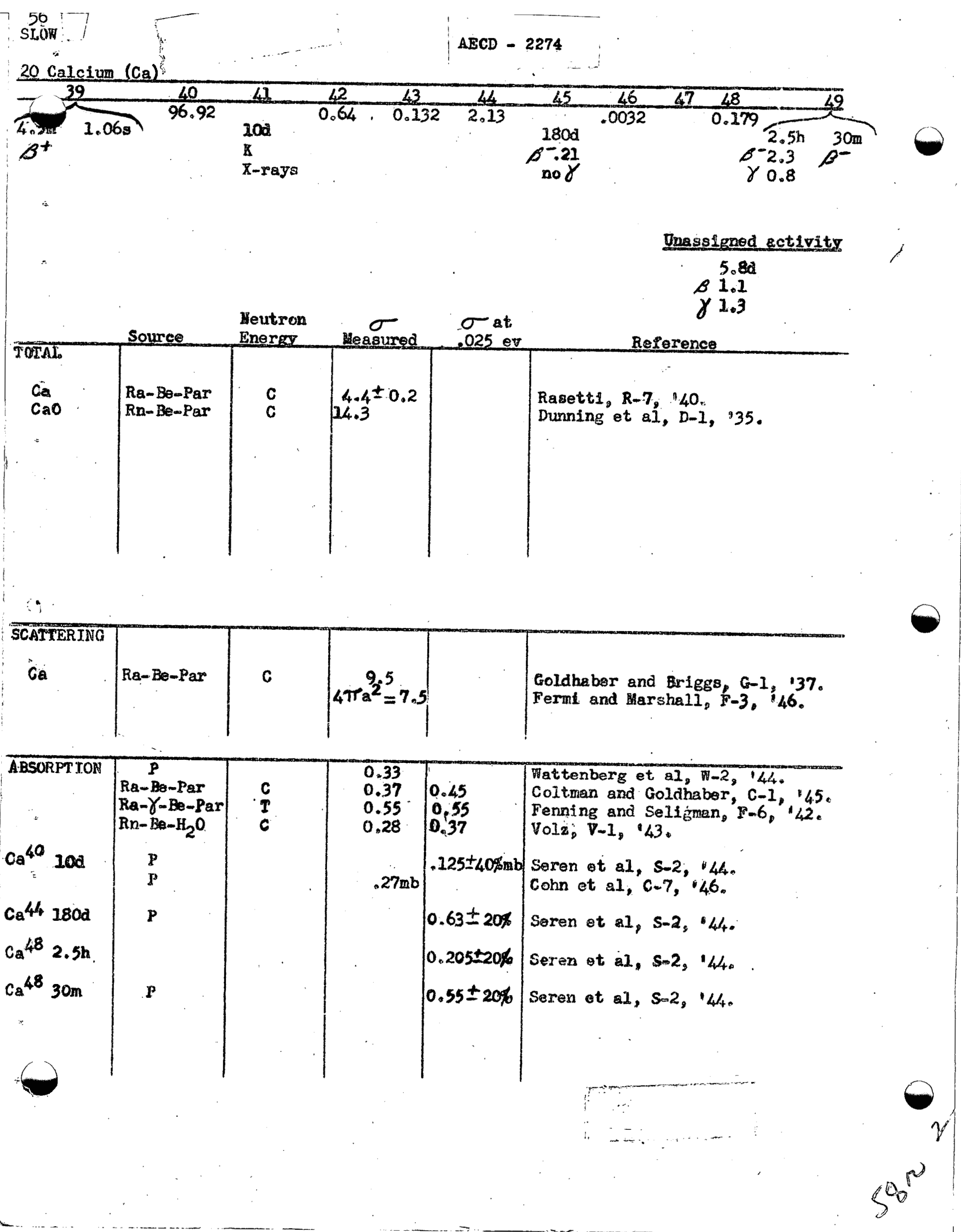




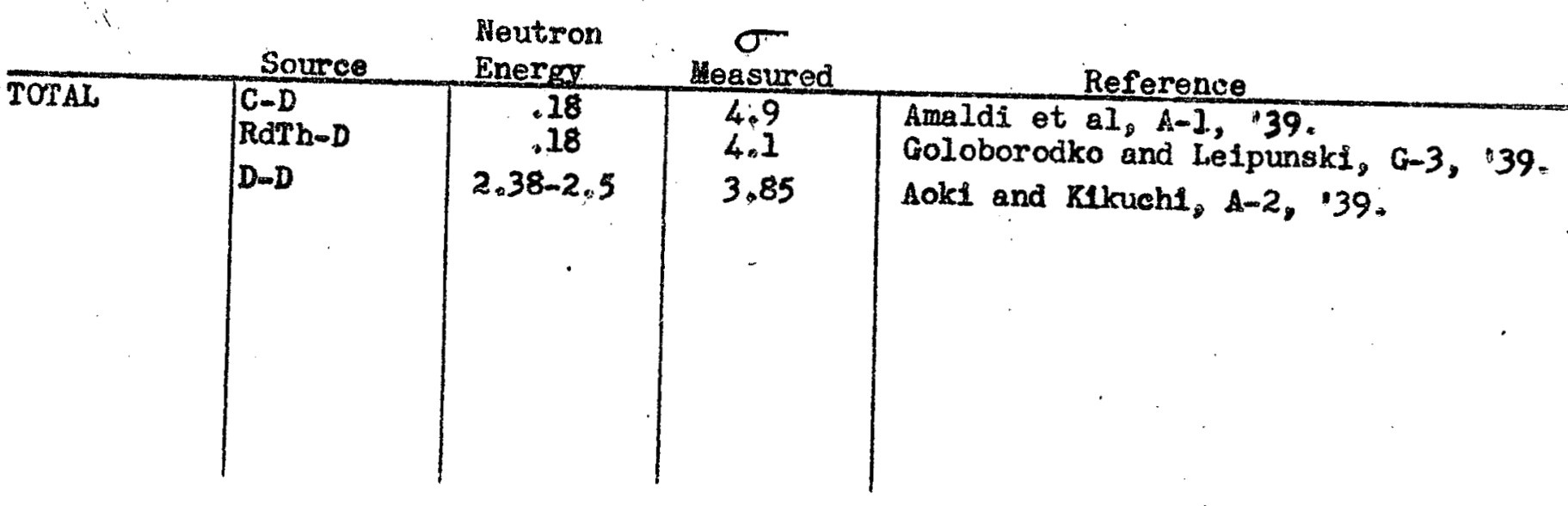
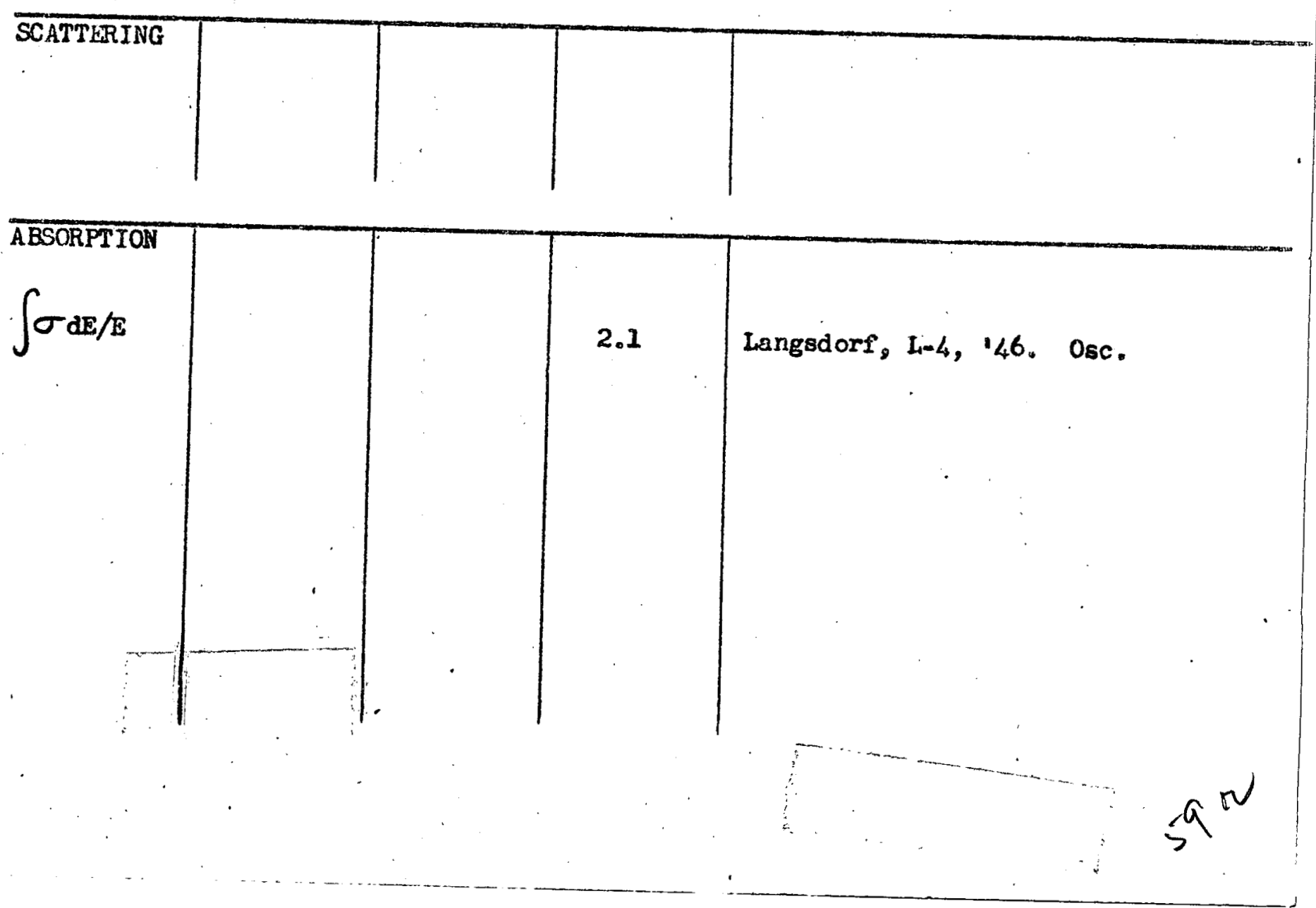


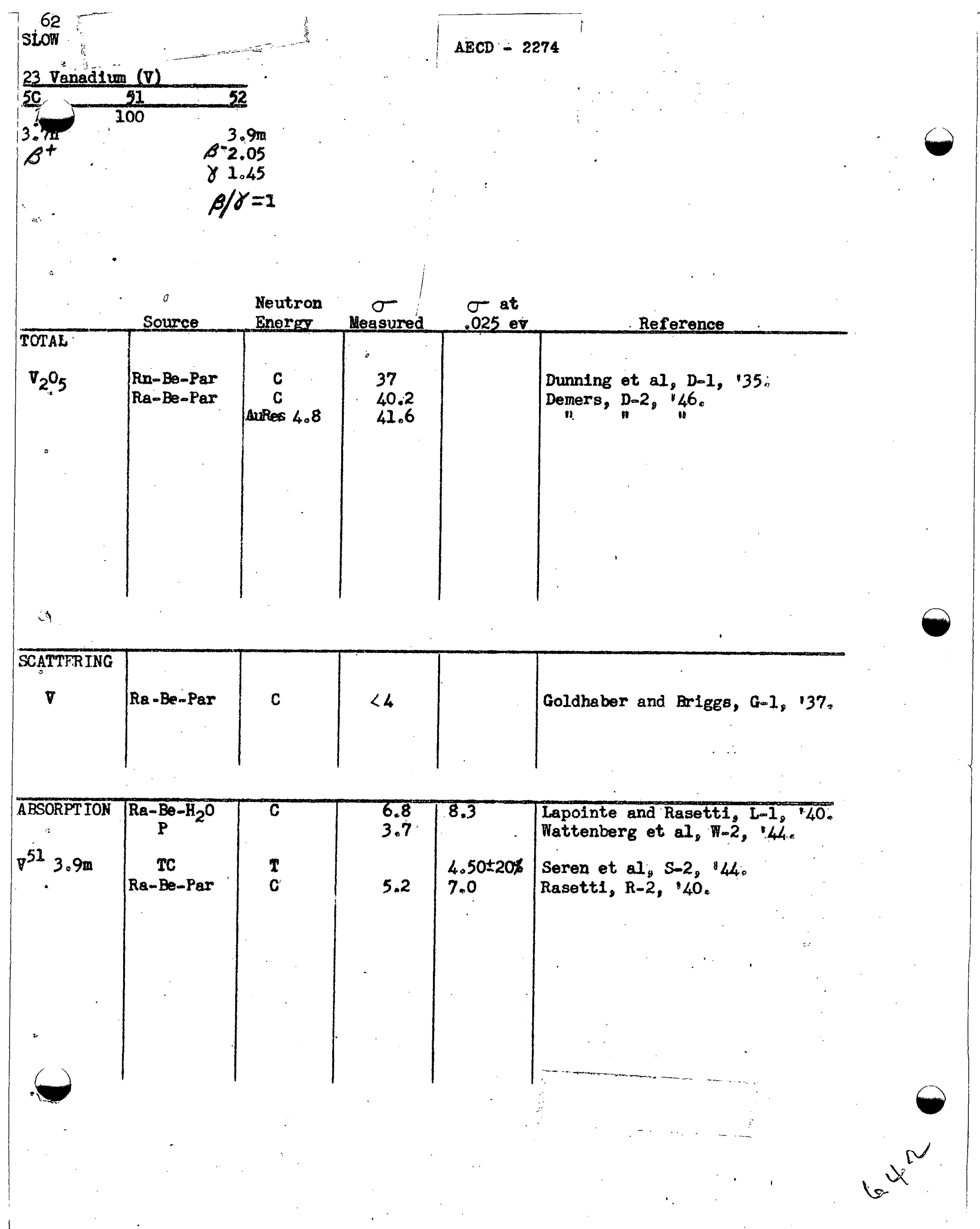




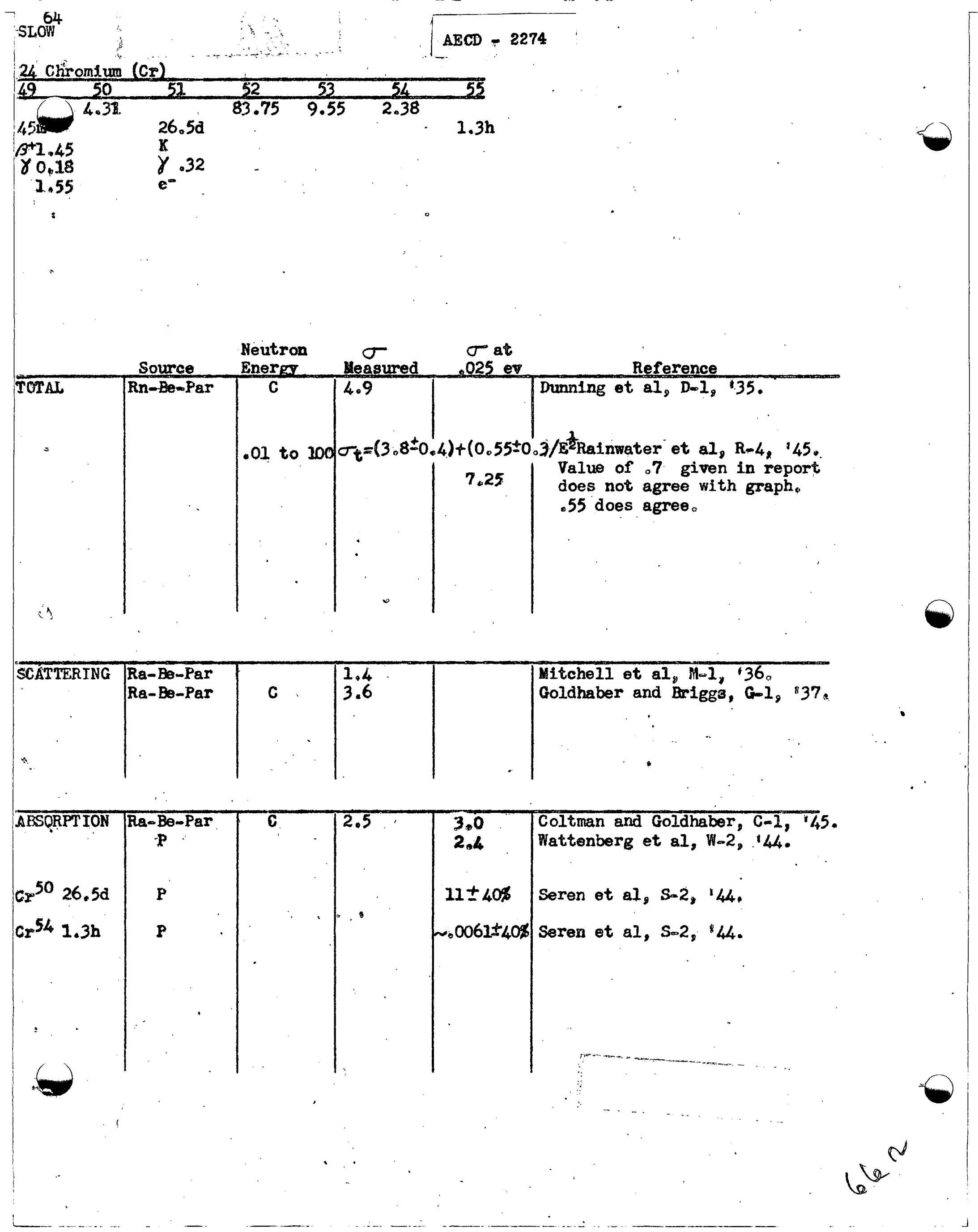




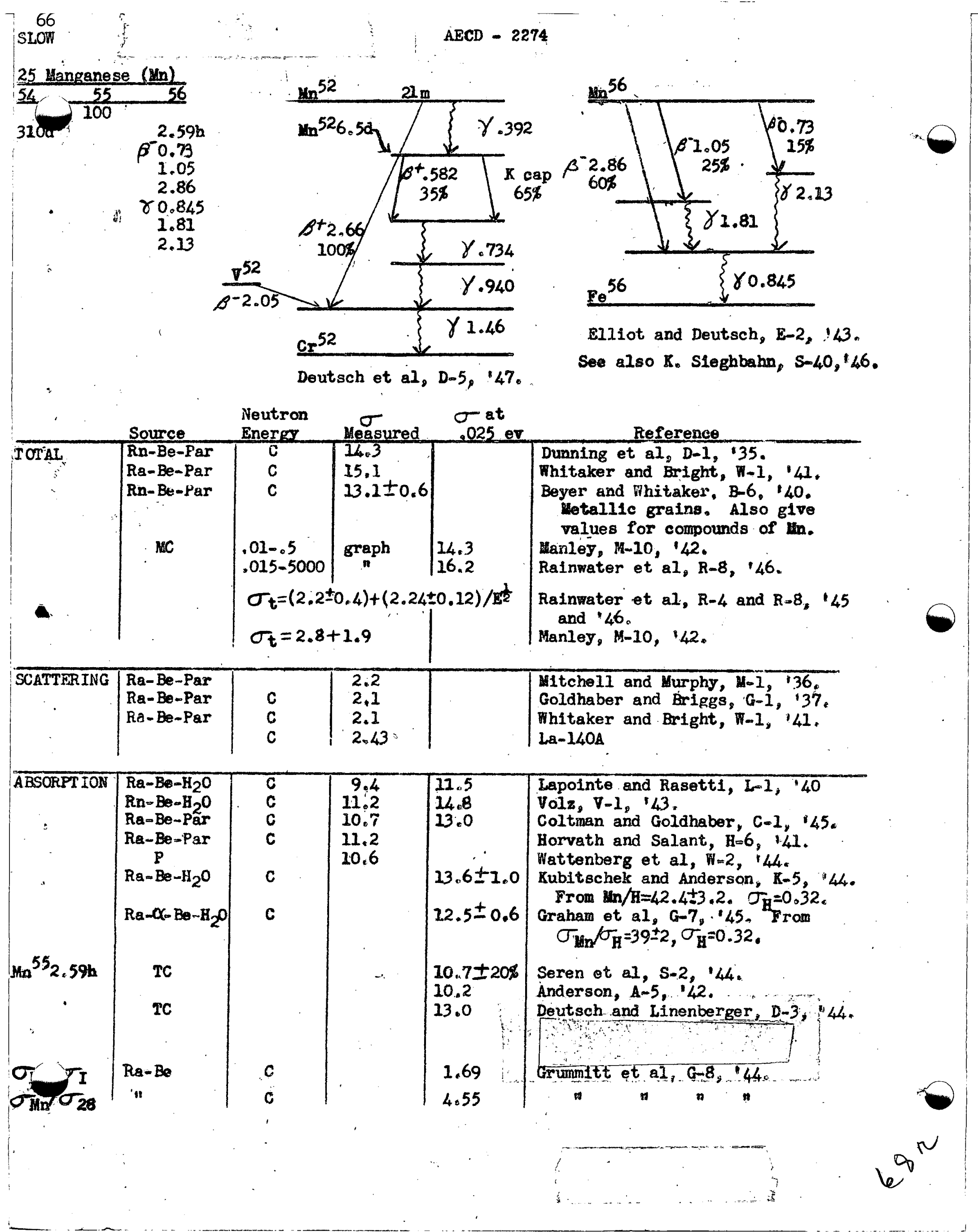




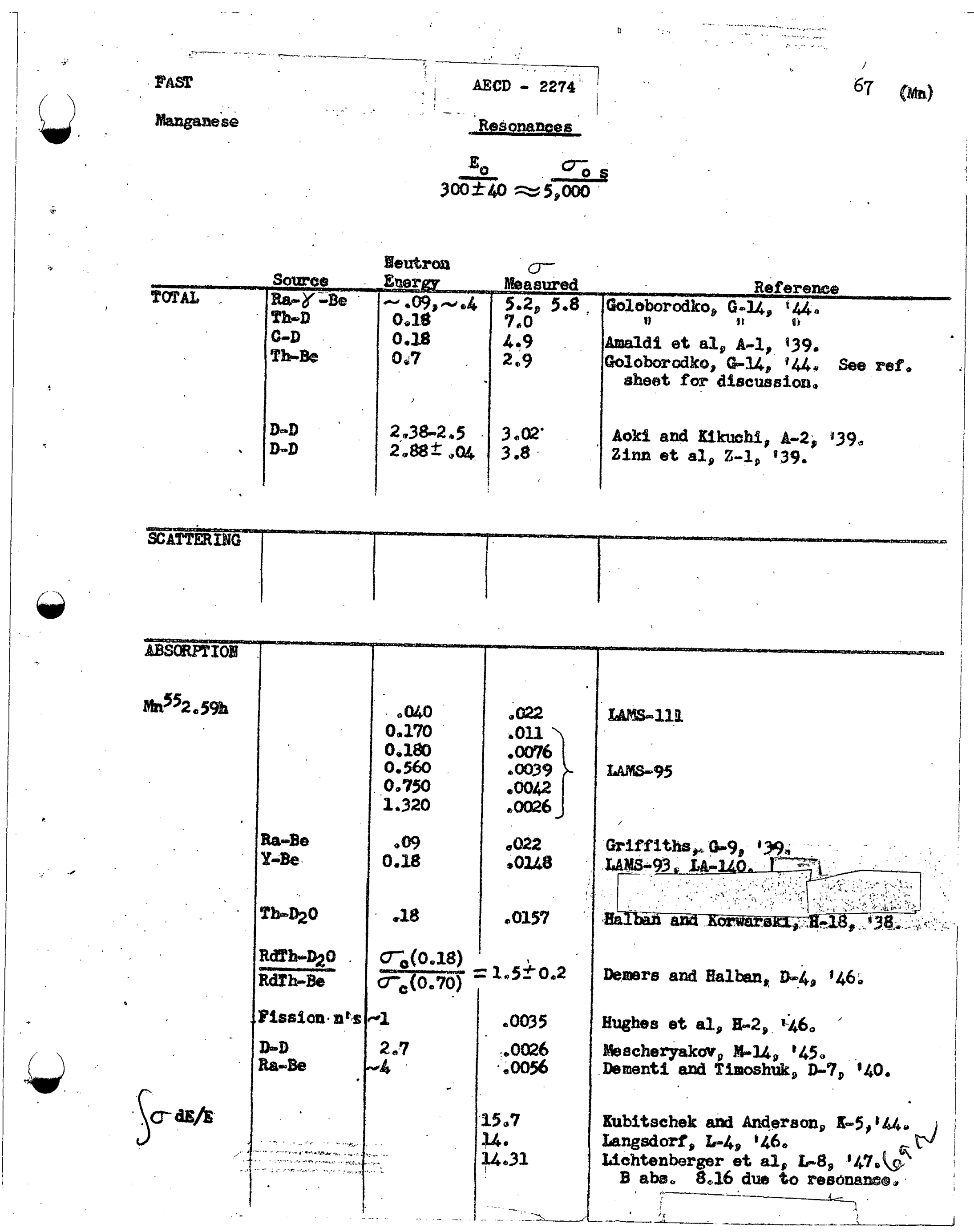




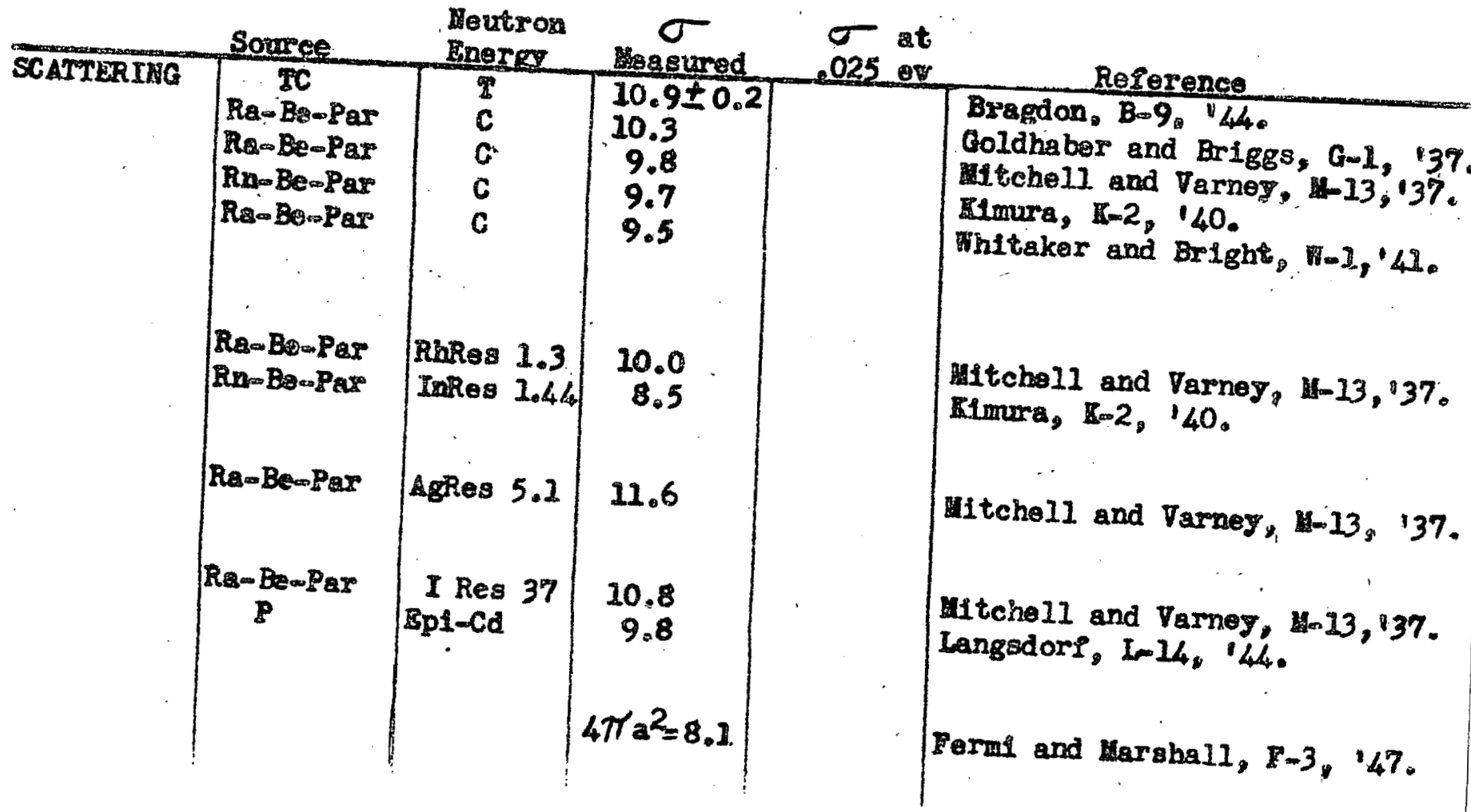

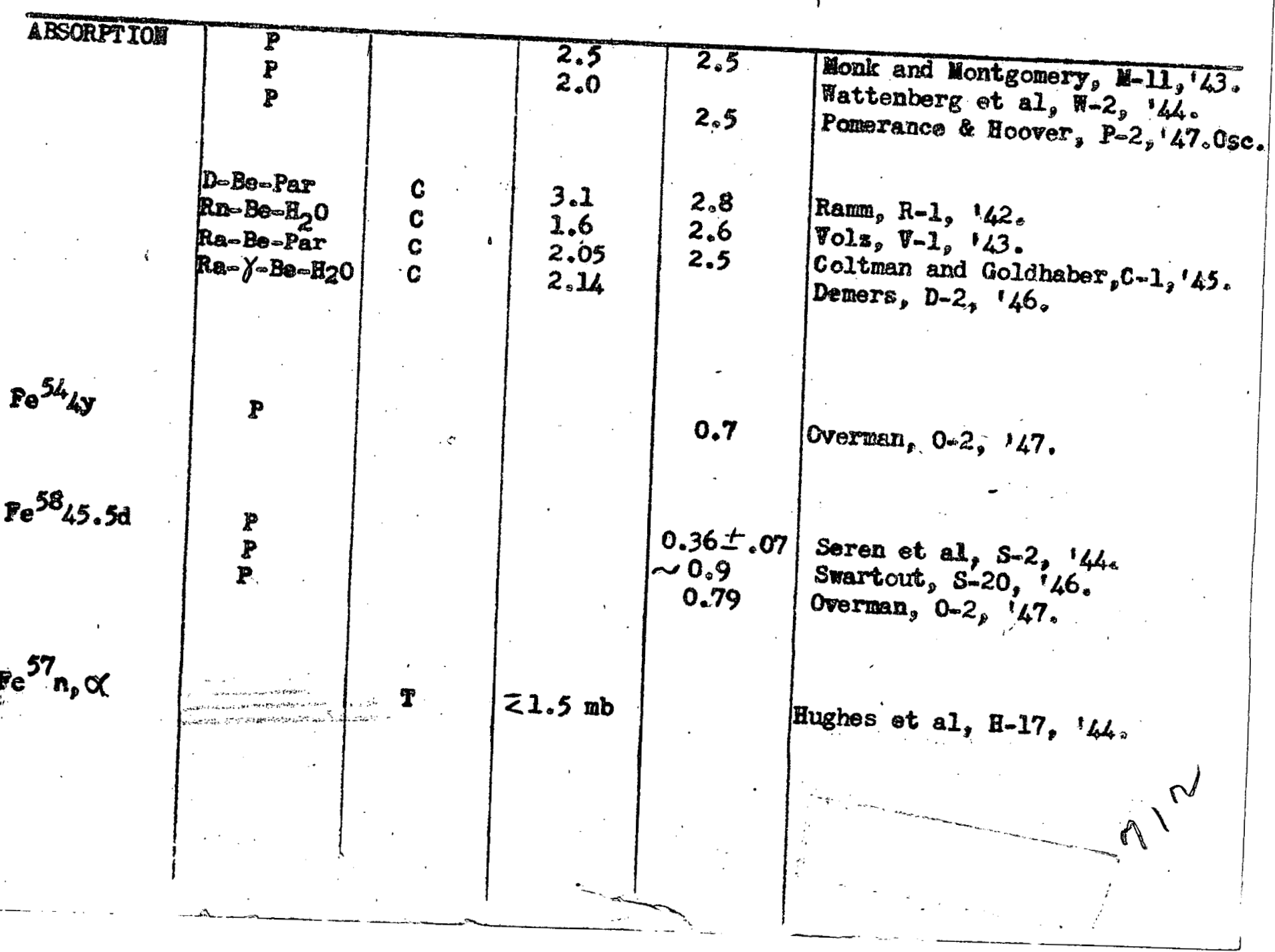




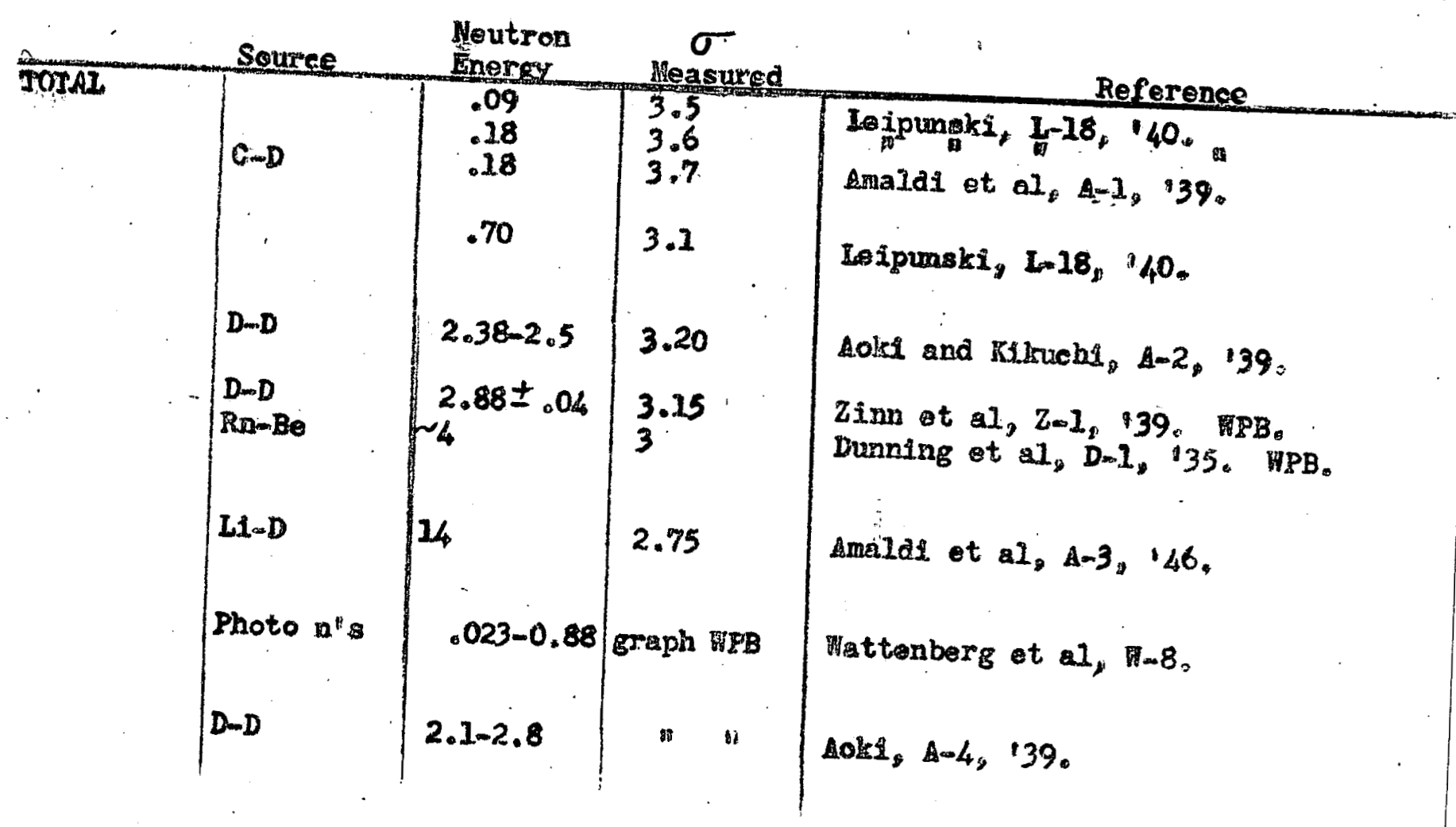
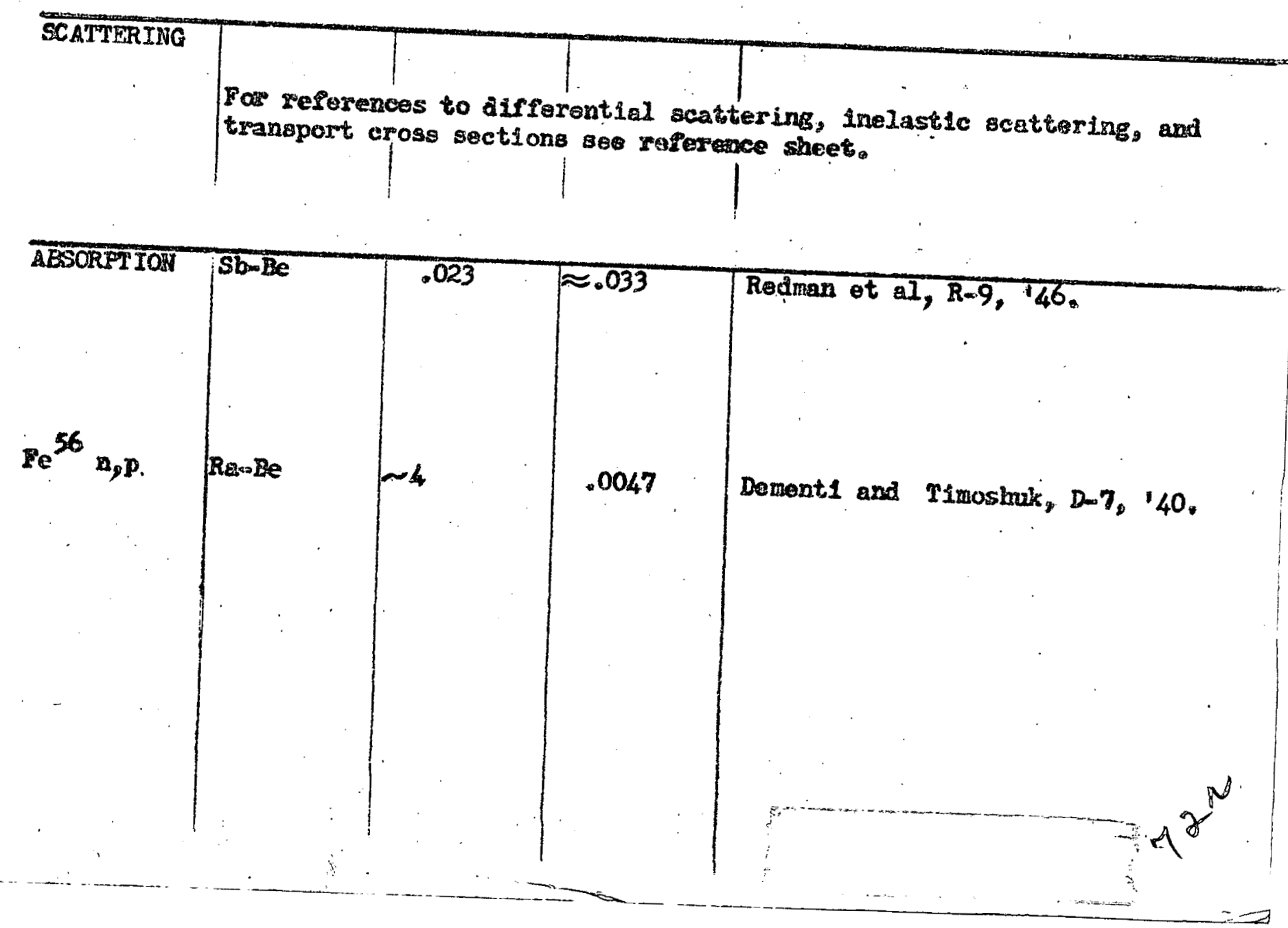


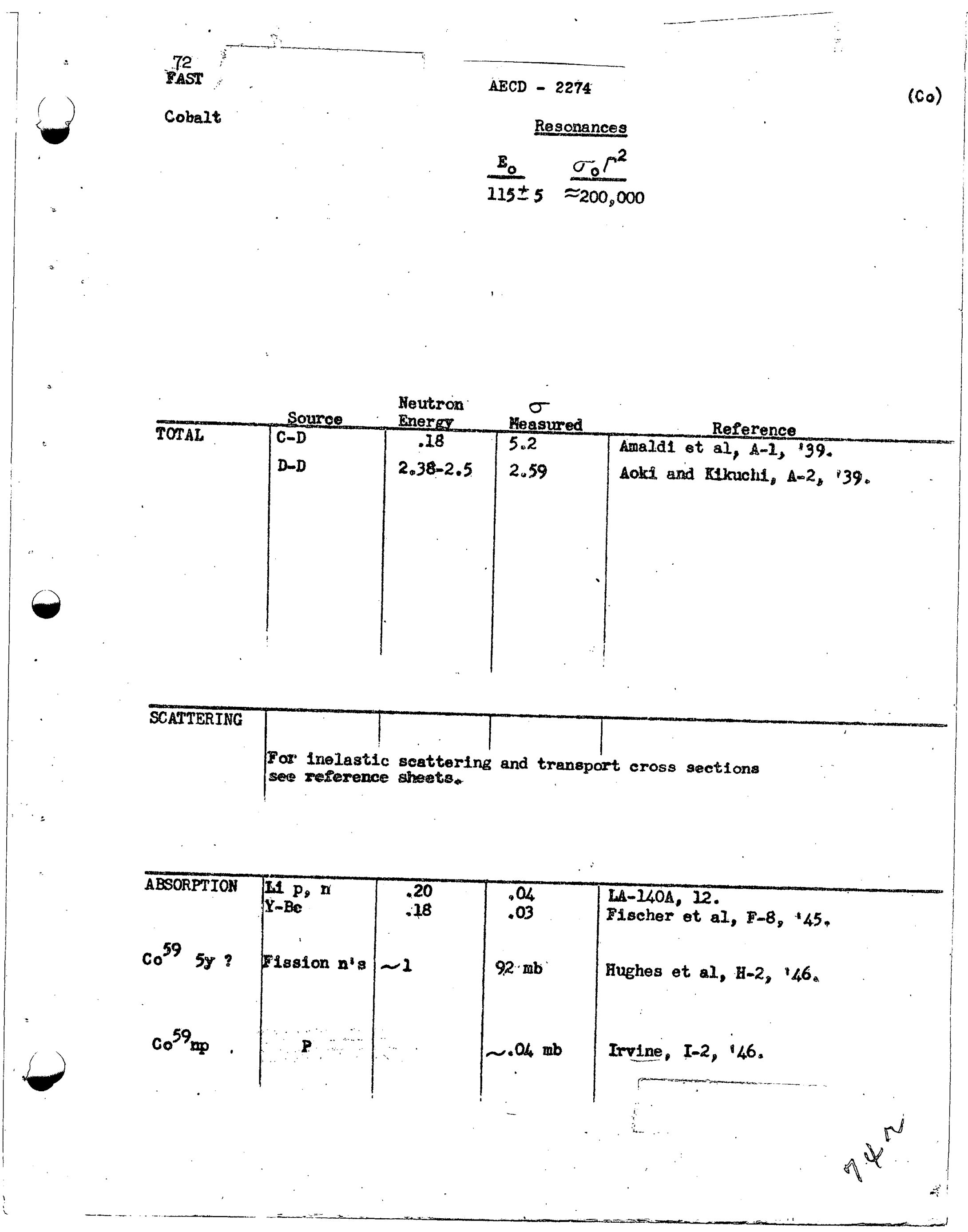


28 Hiokel (N)

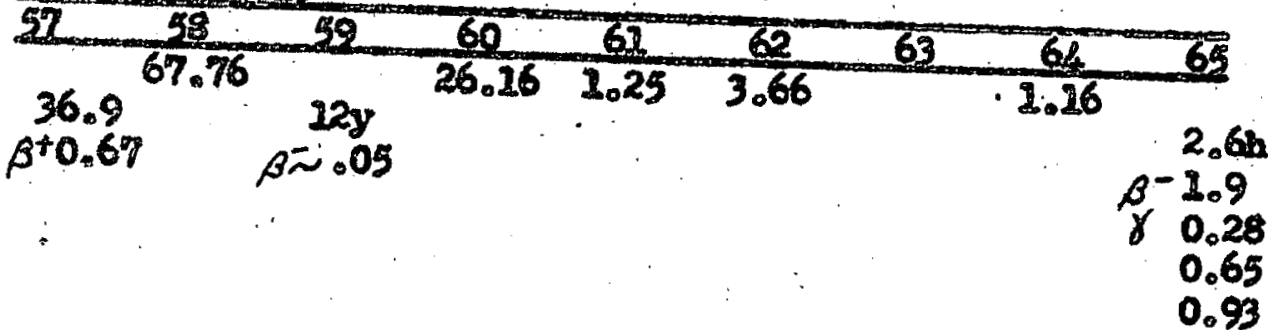

\begin{tabular}{|c|c|c|c|c|c|}
\hline TORA? & Sorres & $\begin{array}{l}\text { Beutrion } \\
\text { Enersay }\end{array}$ & 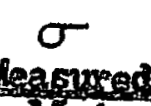 & $\begin{array}{c}\sigma \\
025 \text { at } \\
0.025\end{array}$ & . \\
\hline & $\begin{array}{l}\text { Ras Be-par } \\
\text { Rn-Be-par } \\
\text { Rambe-Par }\end{array}$ & $\begin{array}{l}c \\
c \\
c\end{array}$ & $\begin{array}{l}15.4 \\
29.7 \\
19.8 \\
22.2\end{array}$ & & 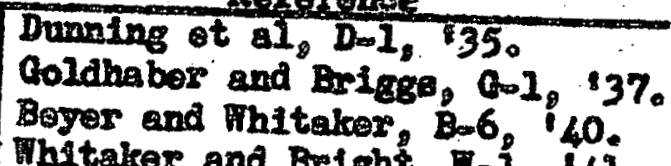 \\
\hline
\end{tabular}

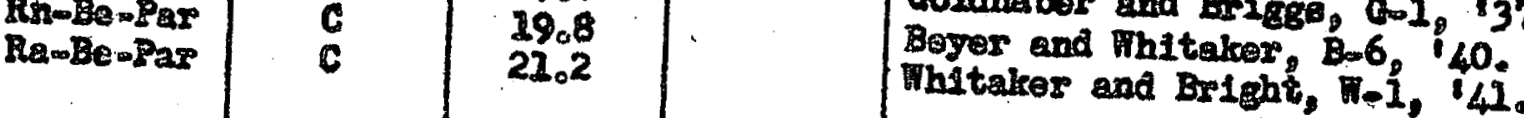

P-Bempar IrRes 10.44 16.1

M $\sigma \approx$ eonst $\approx 18$ .01 to 75 / Eraph WFB

18.5

Hanstein, $\mathrm{H}-4$, $/ 42$. Rolled $\mathrm{H}$ sample.

Rainuater et a1, B.4, 45. 
28 Nickel (Ni) (continued)

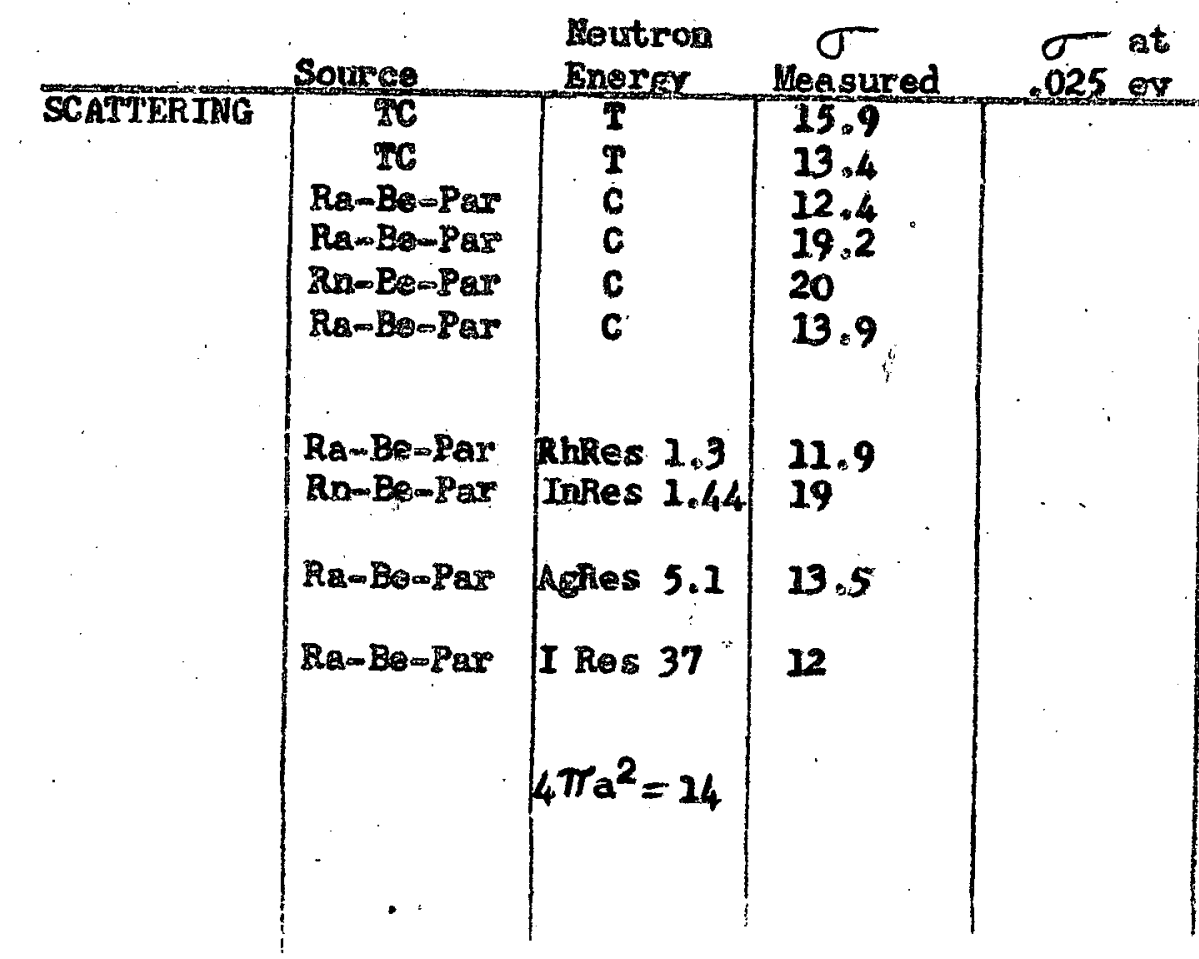

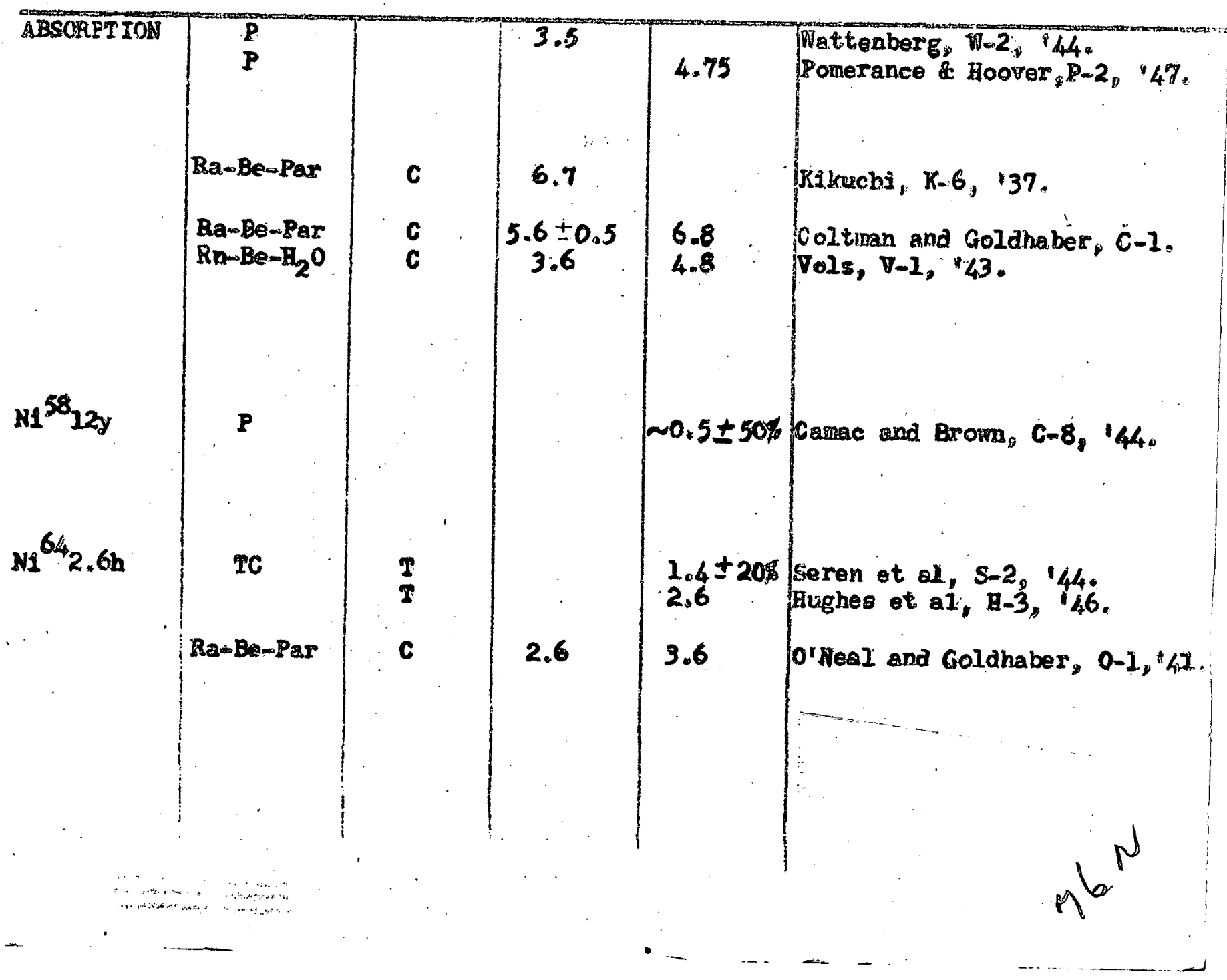


Hekel.

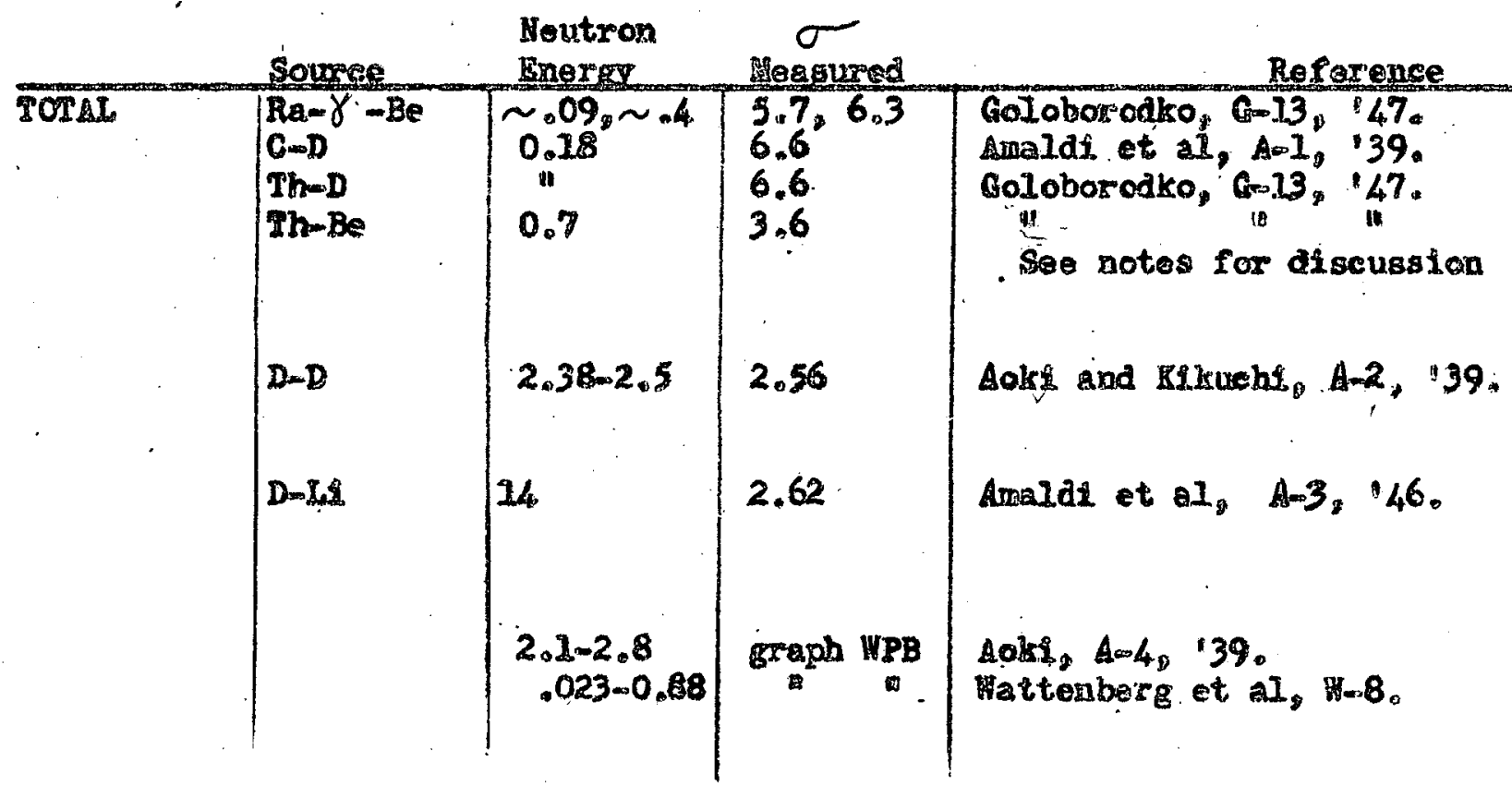
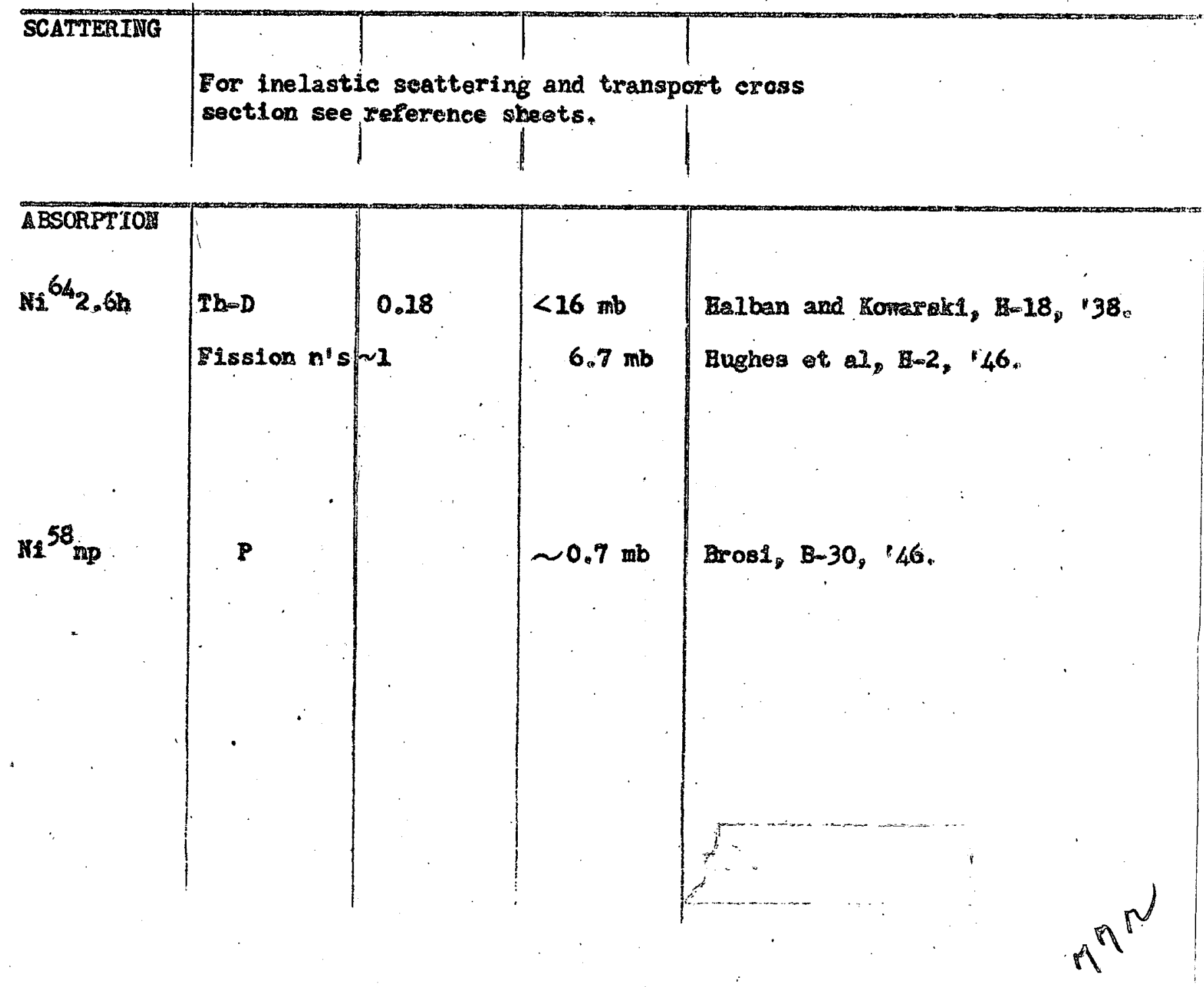

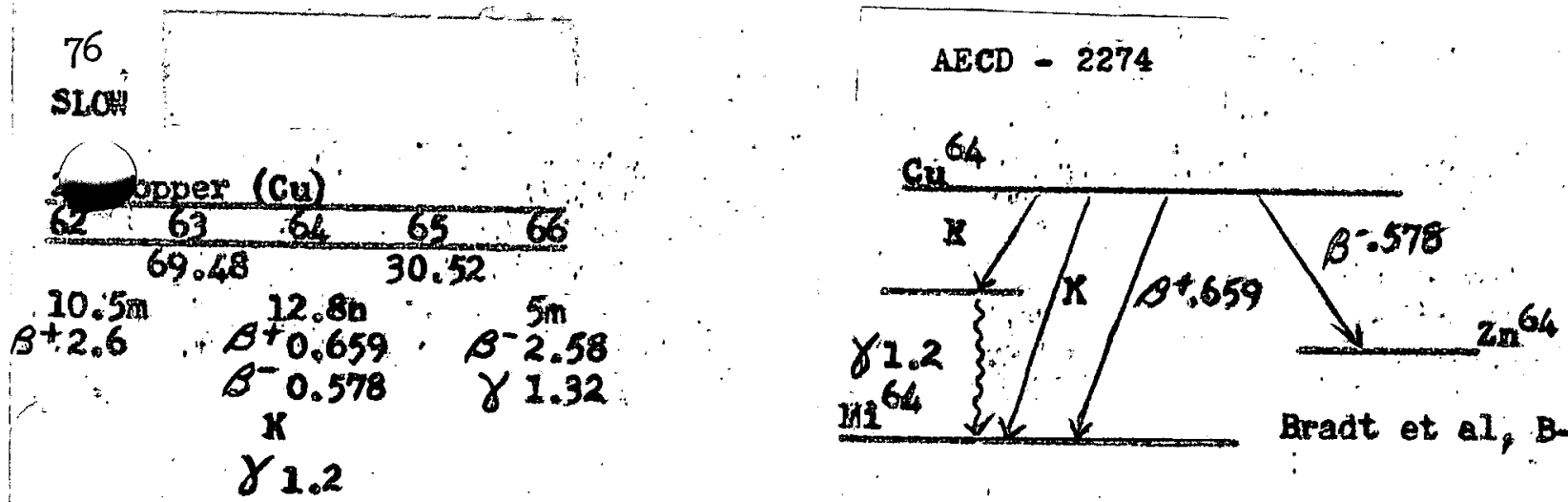

$3_{1}^{64}$ Bradt et al, B-8, $* 1,6$.

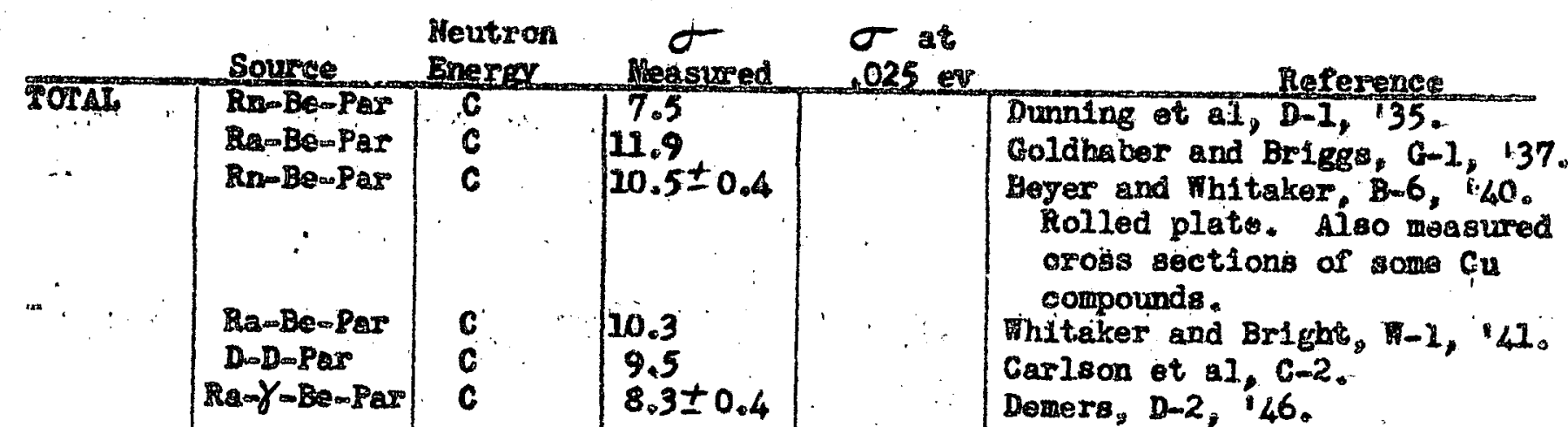

Demers, D-2, 46 .

Ranstain; Ha-4, "4l. Holled Cu.

pole-Par InRes 1.44 8.3 8.0 .3

Domers, D-2, 46 .

Ra-X-Be-Par Autes" $4.8,9.4 \pm 0.6$

, 46.

10.6 Rainwater et al, $R-4 ; 45$.

\section{.015-100 graph WPB}




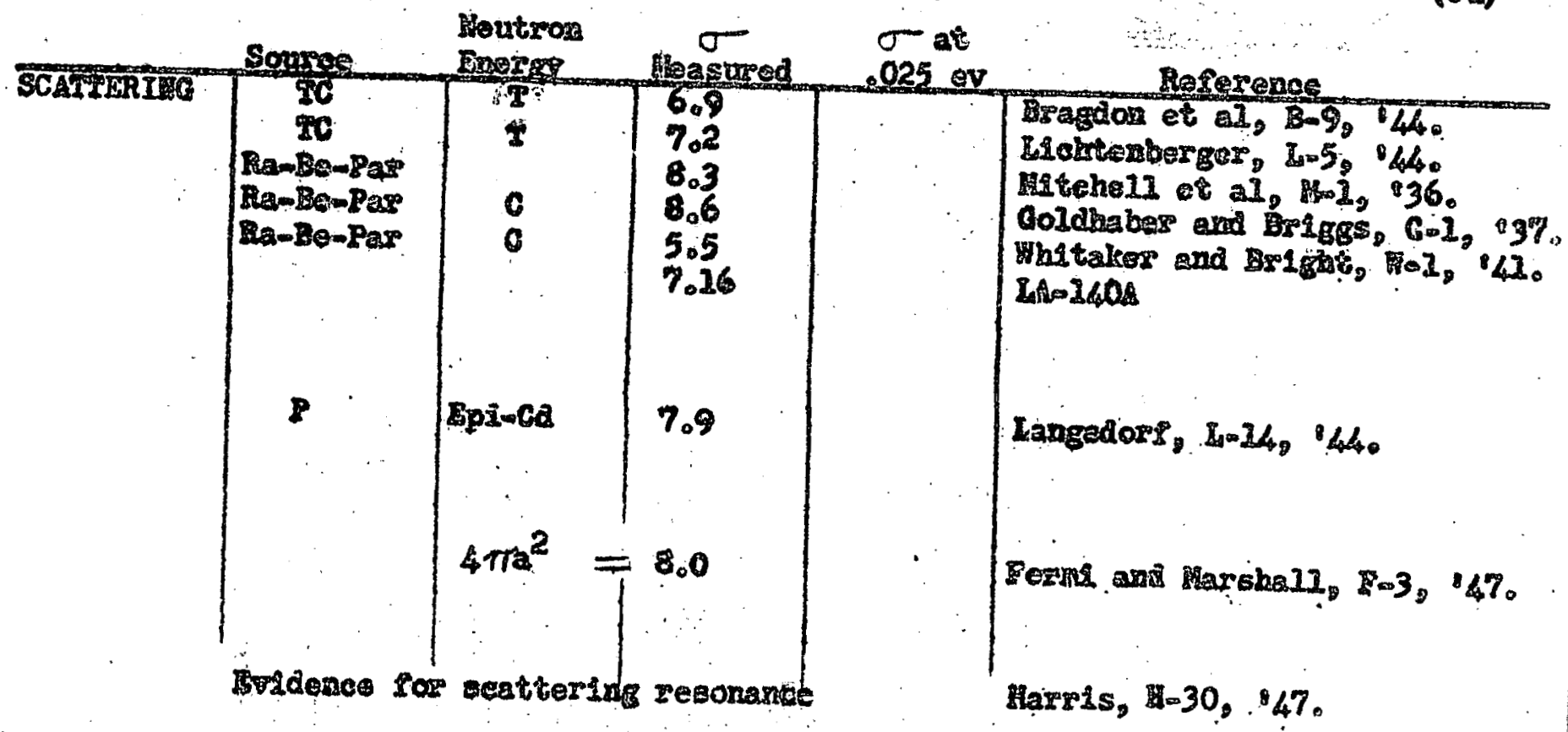

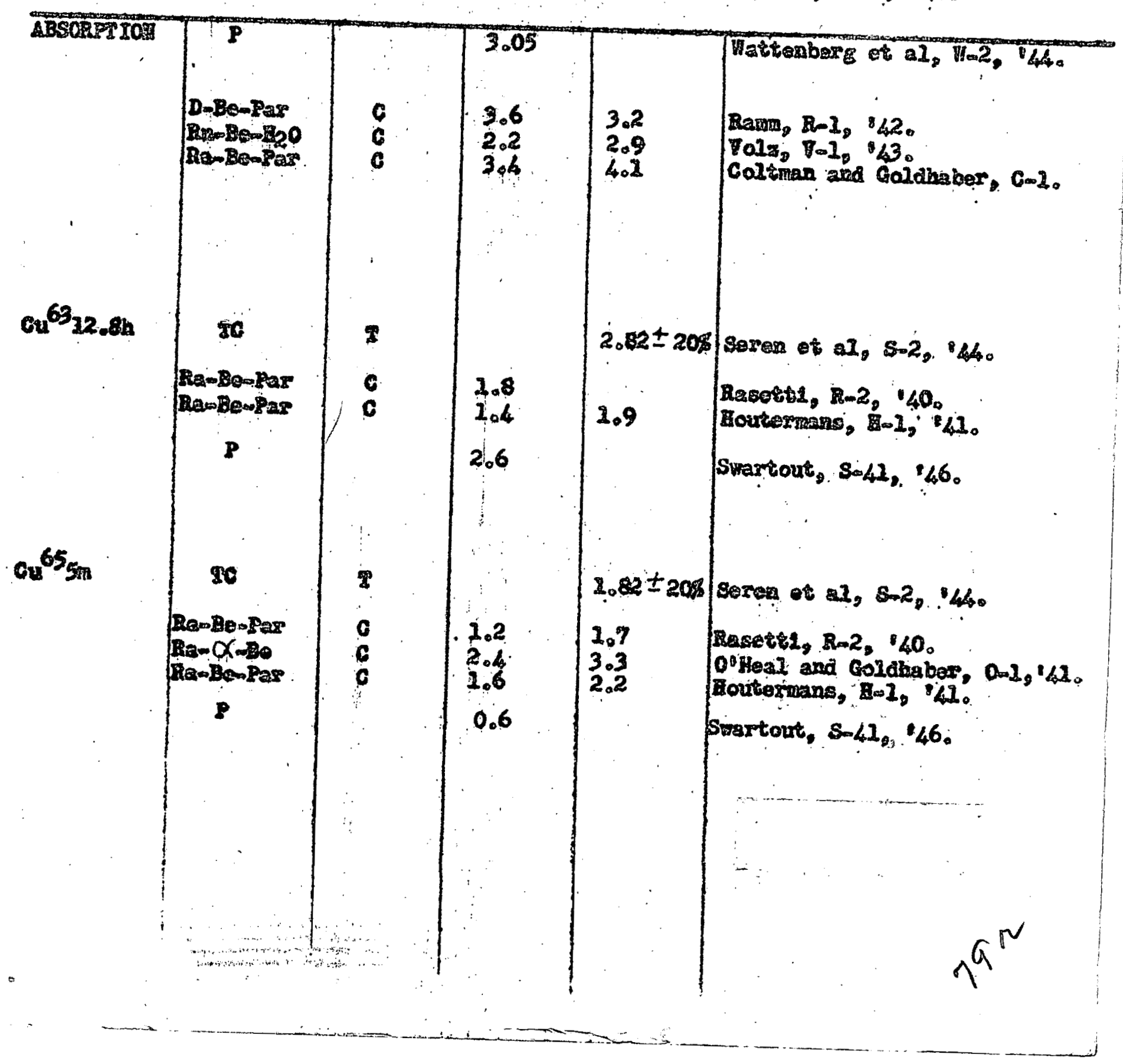


78

, FASA

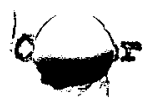

$\mathrm{Cu}^{65}$

$$
\text { AECD - } 2274
$$

hasonaneas

$$
\frac{0.36}{0.65} \text { of posstblo }
$$

570

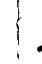

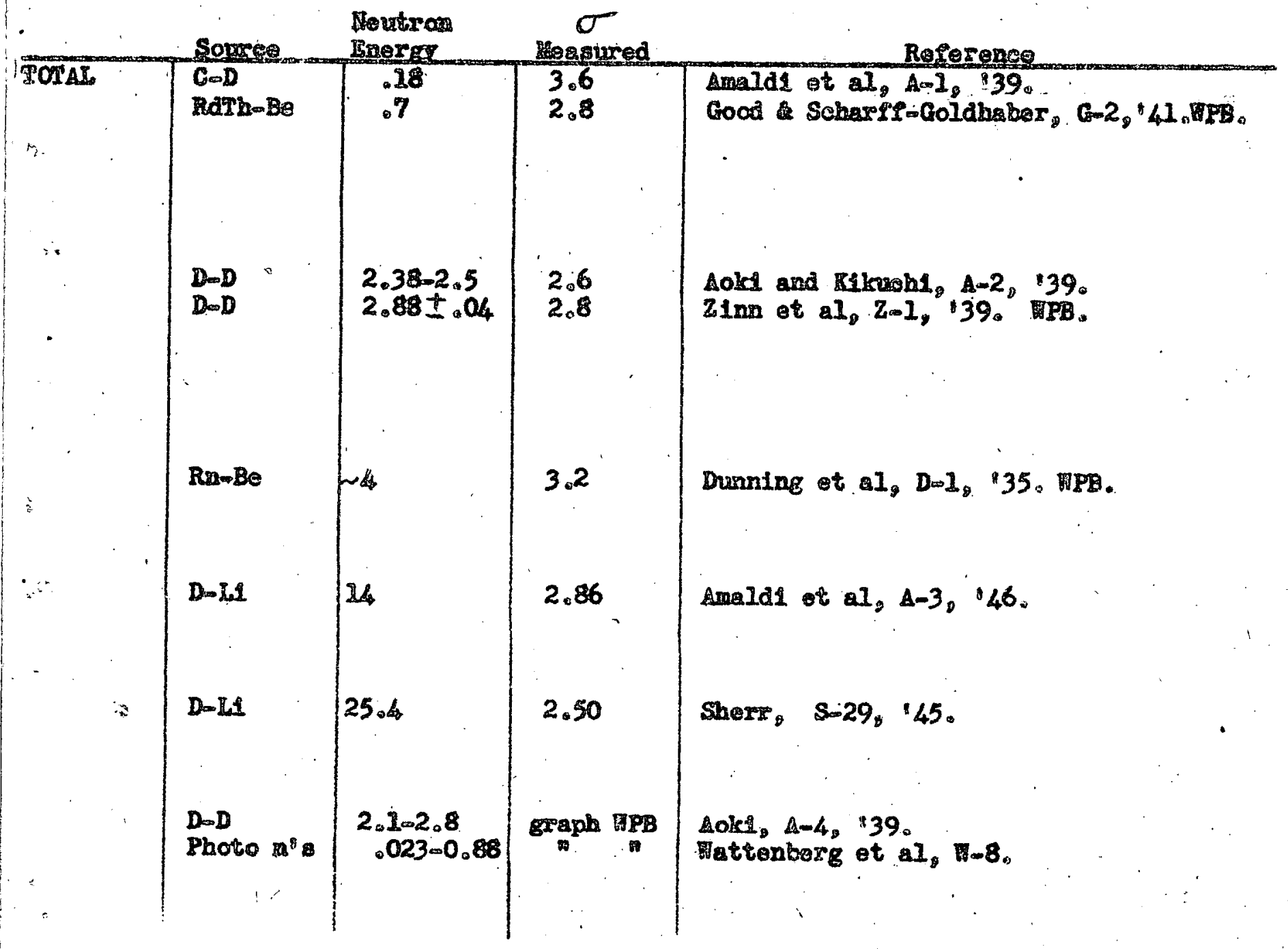

$$
\text { SCATFIRIHG: }
$$

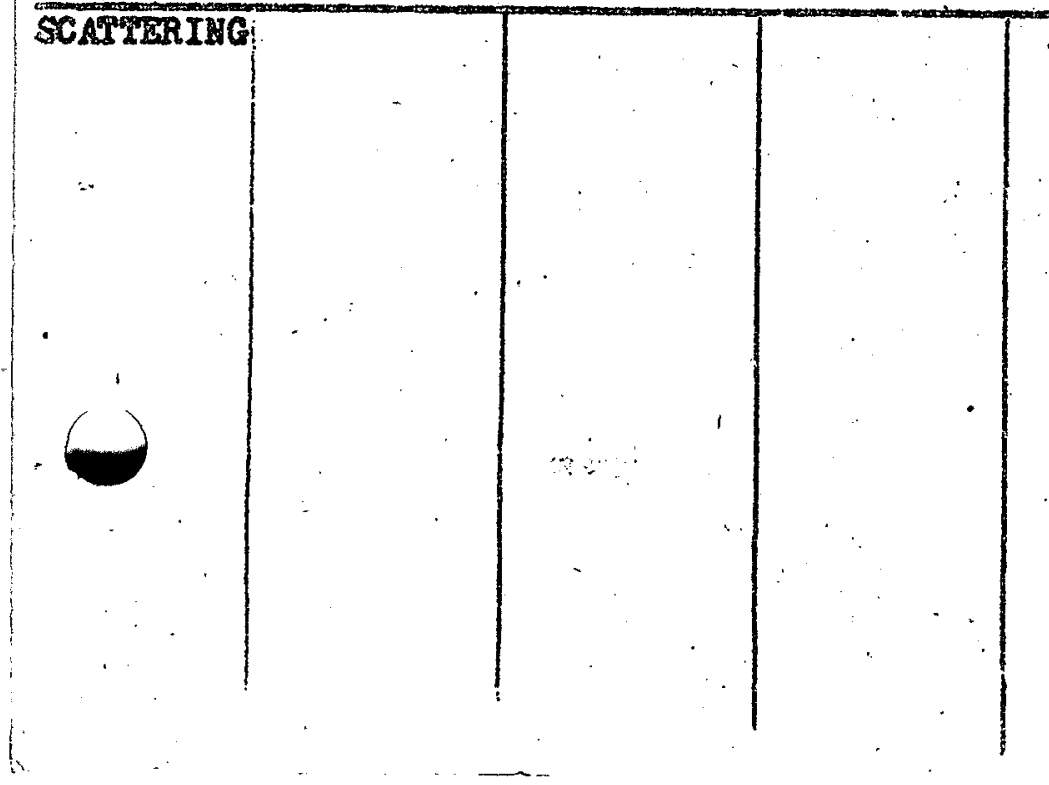


Coppes. (Contimued)

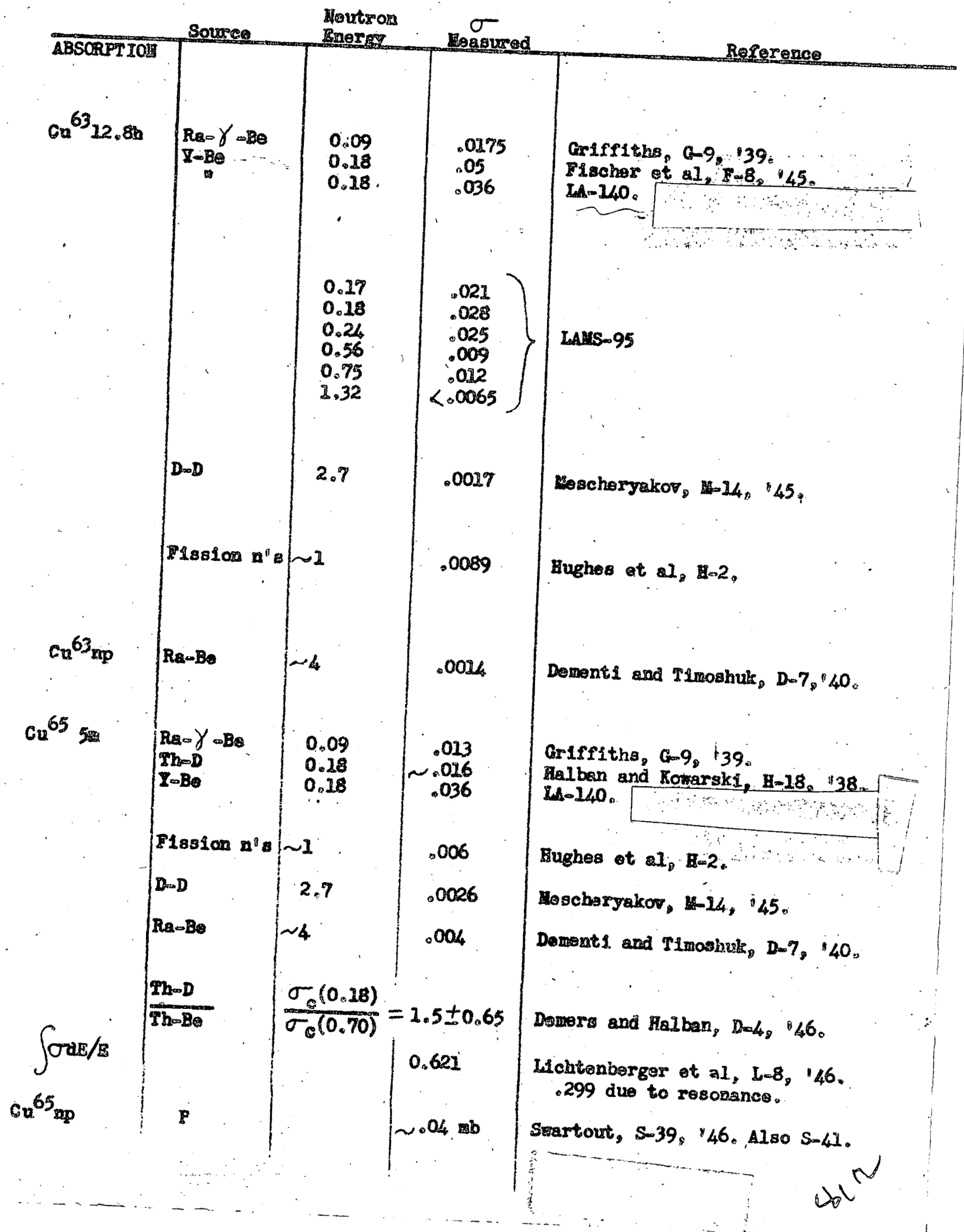




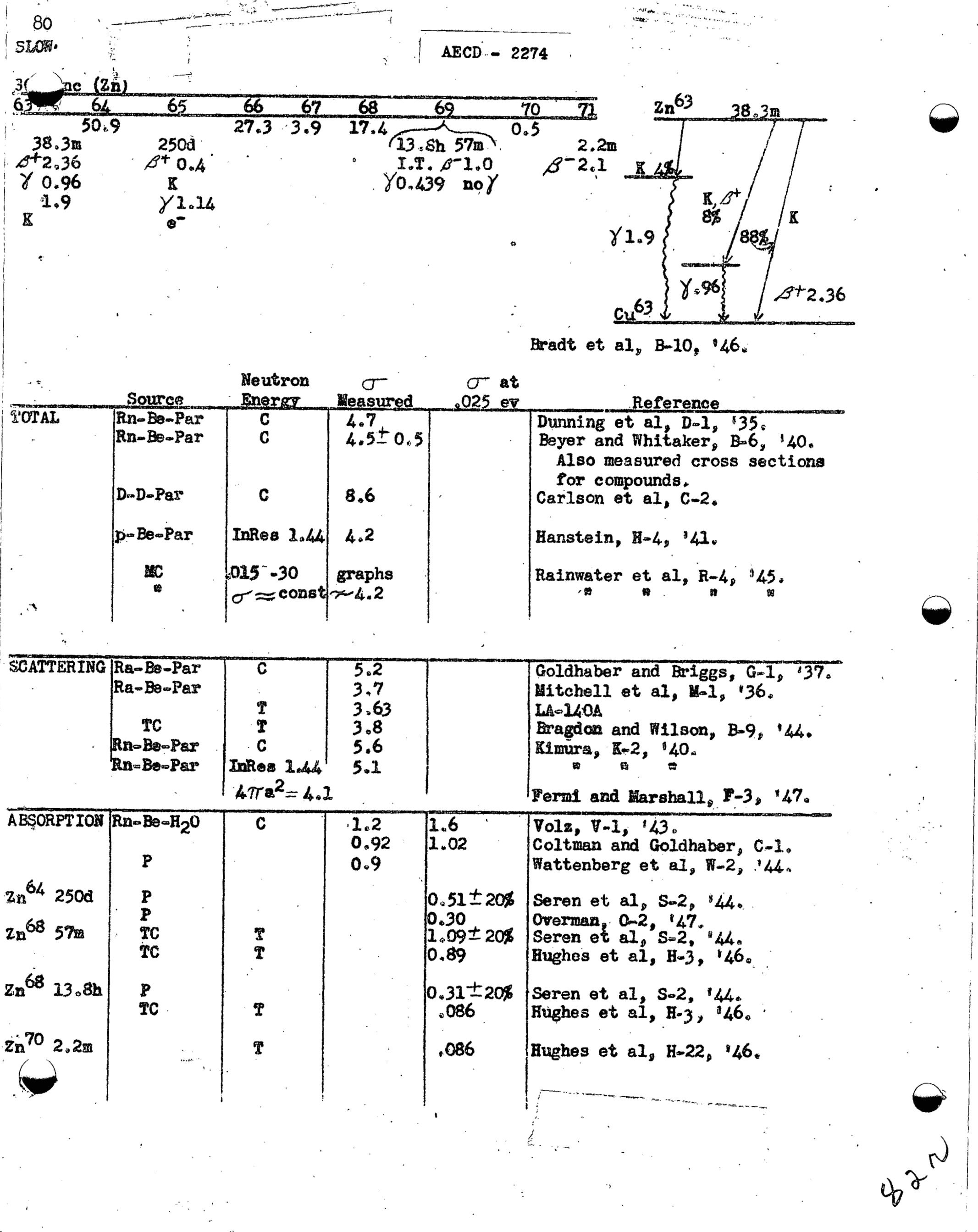


Zine

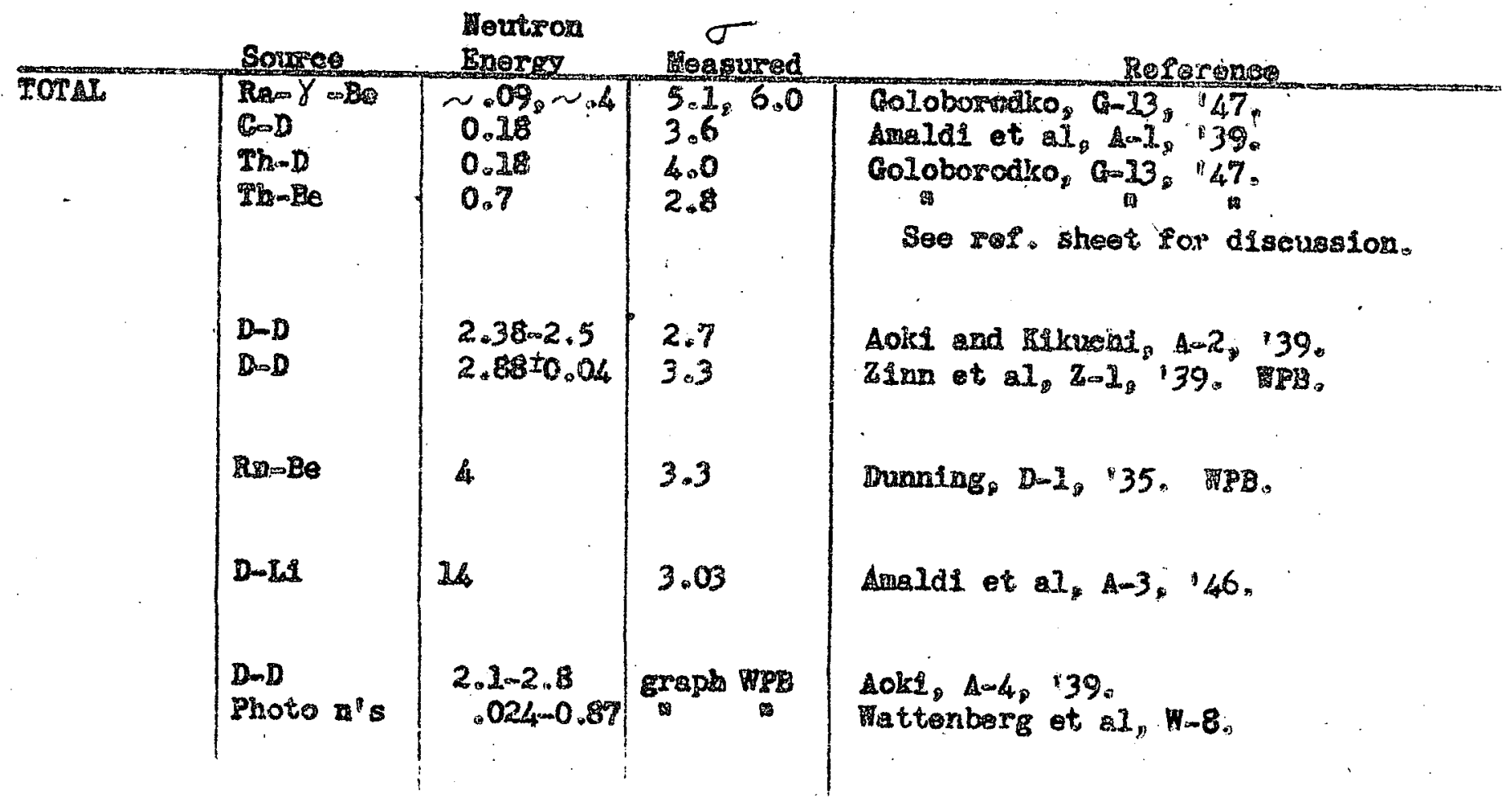

SCATTERTIR

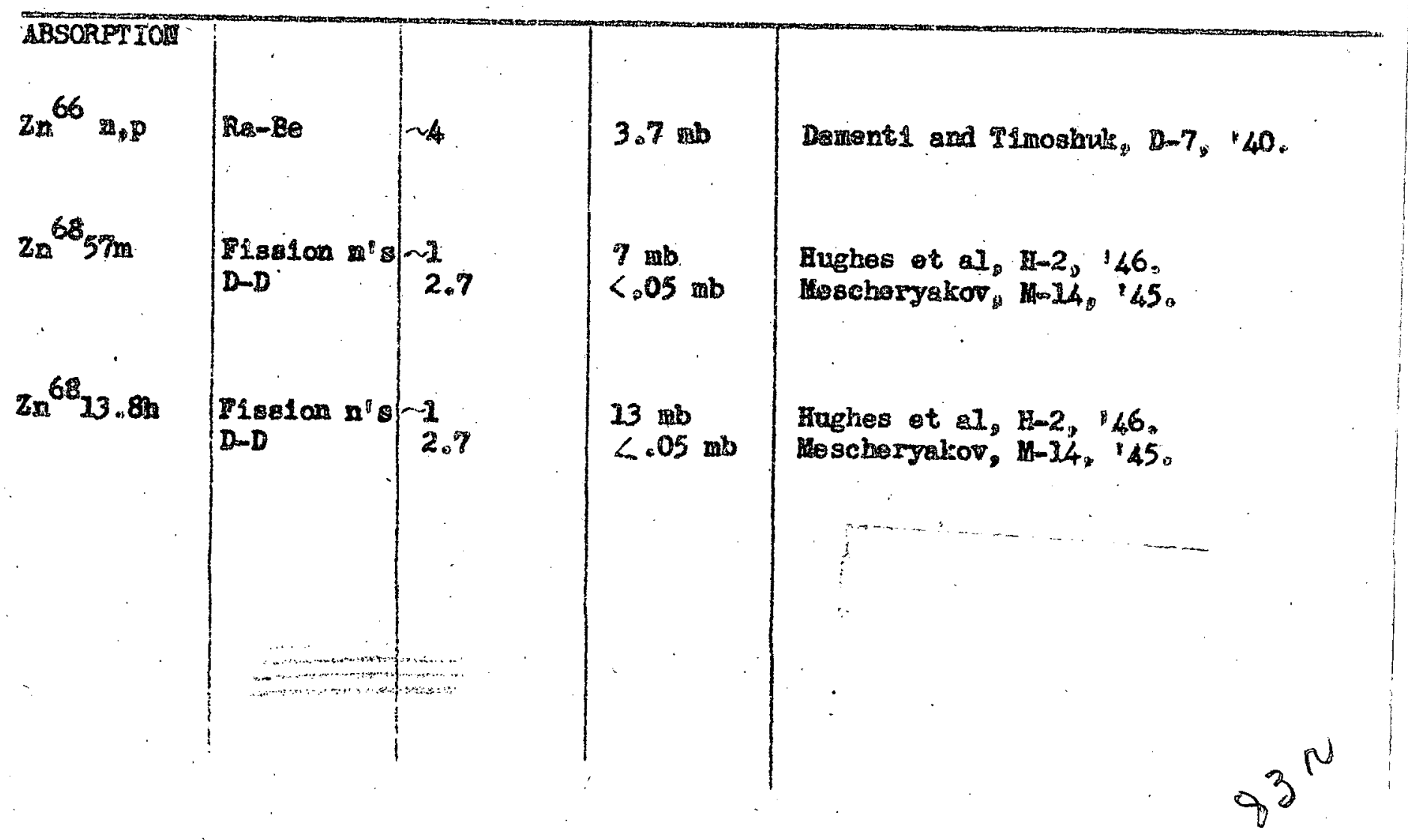




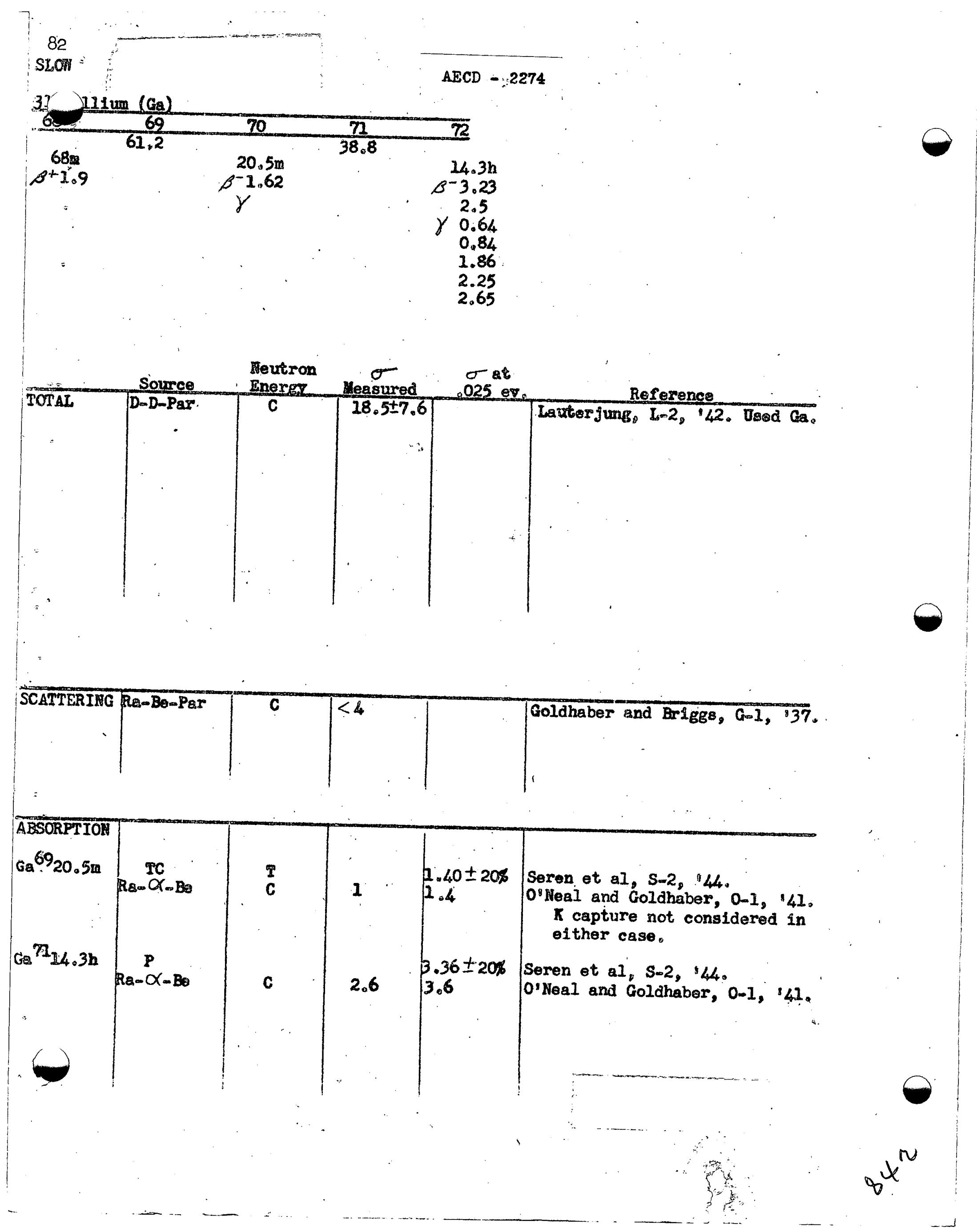



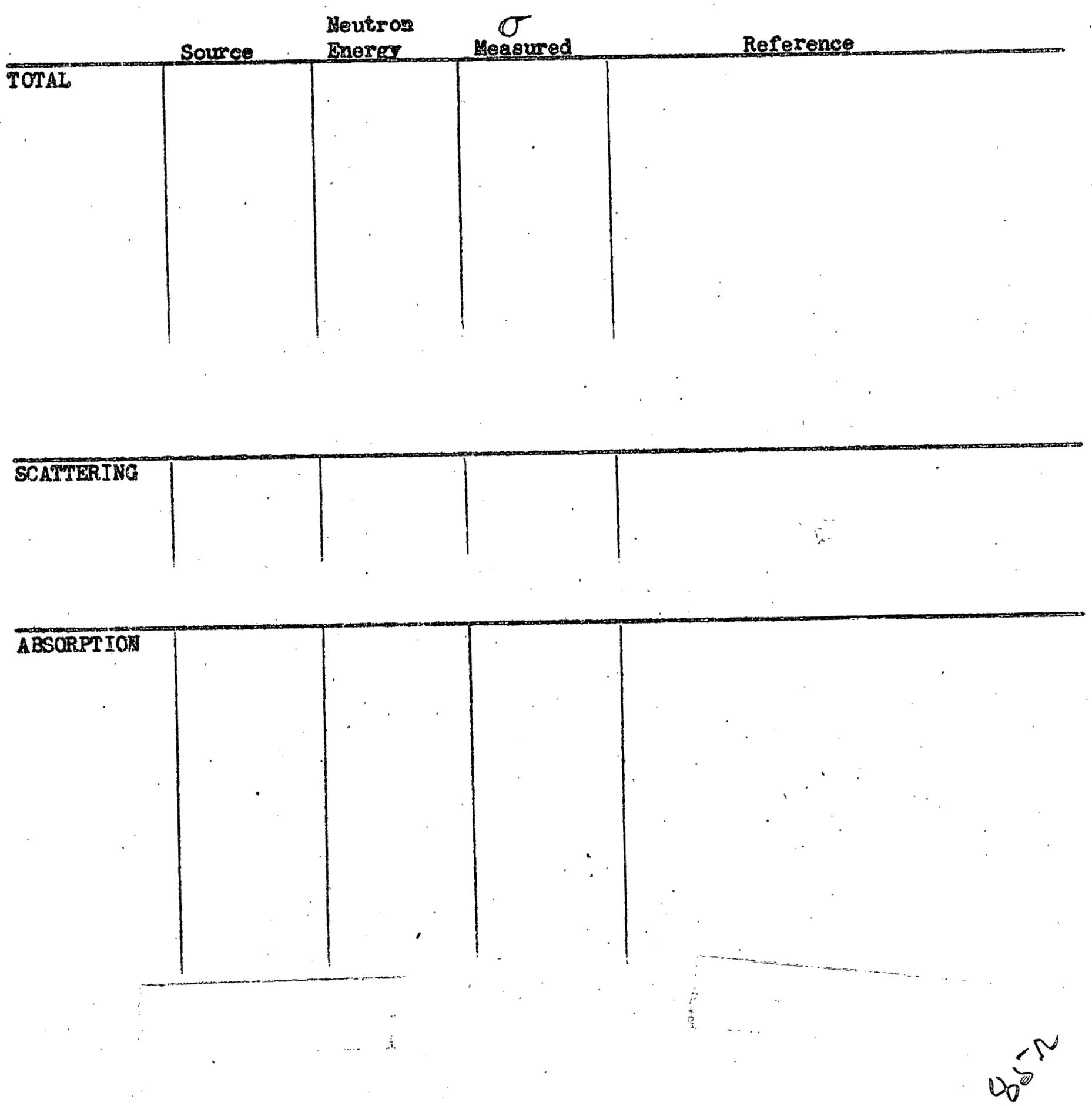

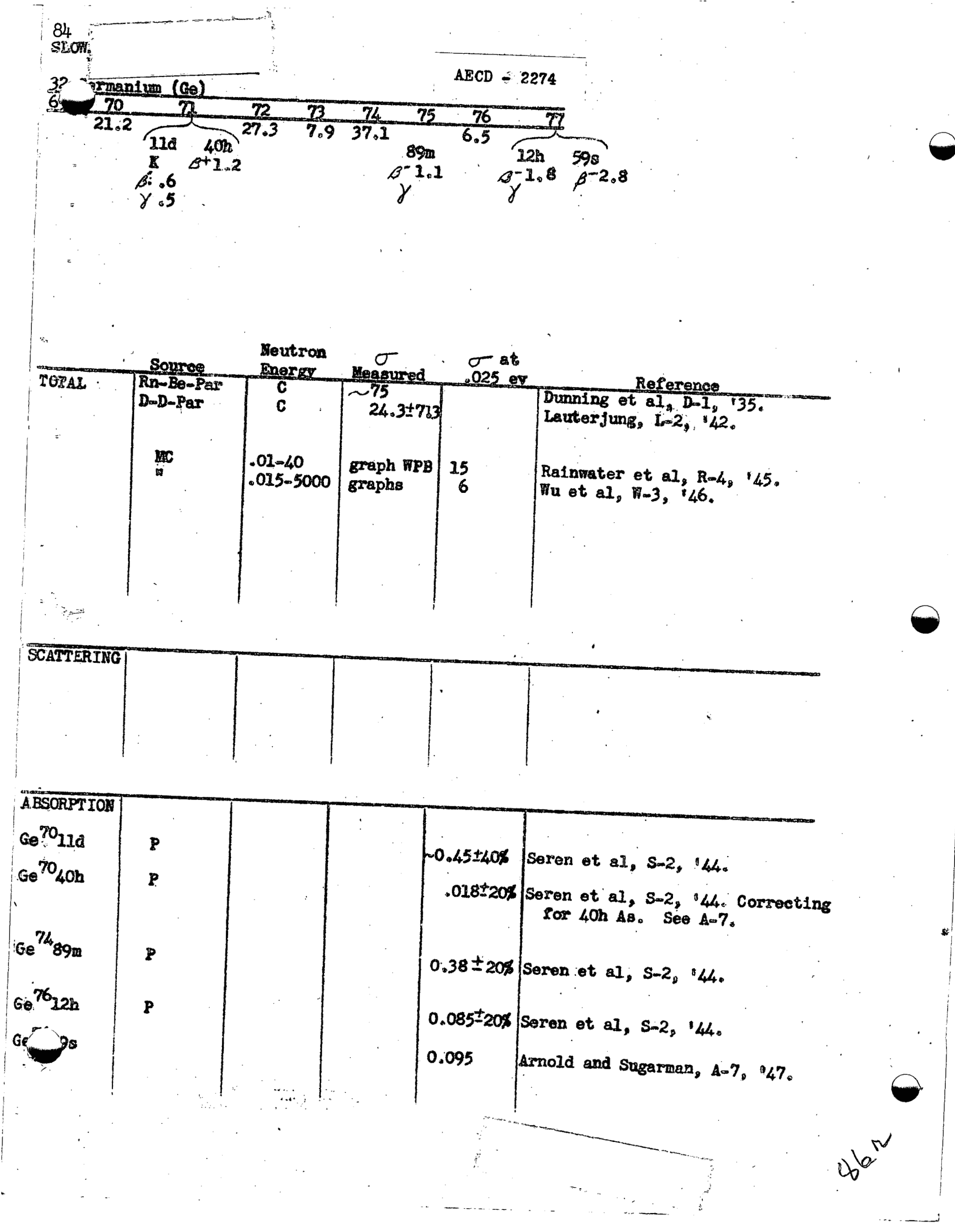


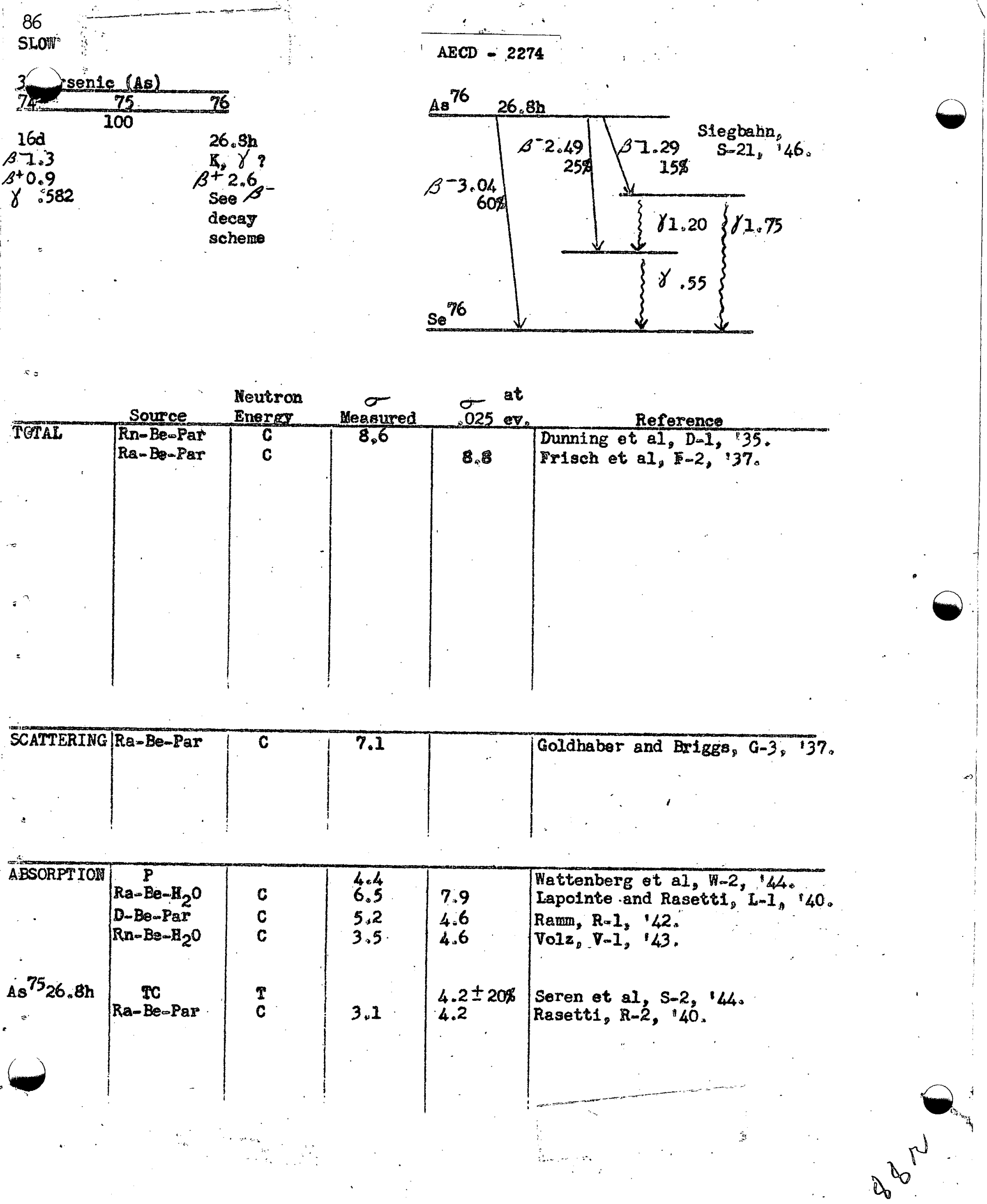



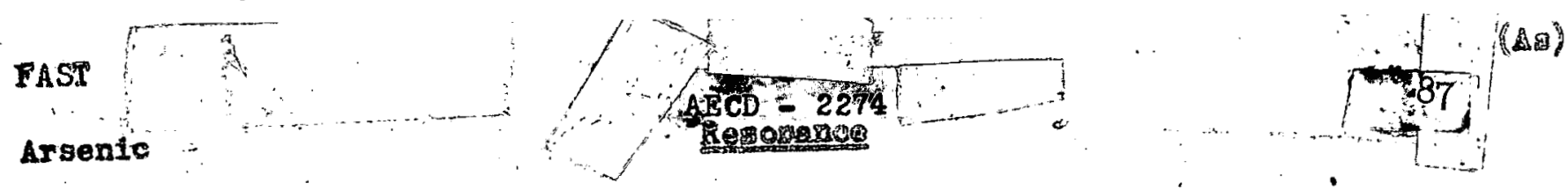

$$
\frac{E_{0}}{\sim 30}
$$

\begin{tabular}{|c|c|c|c|c|}
\hline & Souree & $\begin{array}{l}\text { Neutron } \\
\text { Eneroy }\end{array}$ & $\underset{\text { Heasured }}{\sigma}$ & Referenee \\
\hline TOTAL & $\begin{array}{l}\operatorname{Ra}_{C-D}-\gamma-B e \\
C-D \\
T h-D\end{array}$ & $\begin{array}{l}0.09,0.4 \\
0.18 \\
0.18\end{array}$ & $\begin{array}{l}8.2,7.0 \\
4.7 \\
6.3\end{array}$ & $\begin{array}{l}\text { Goloborodko, } G-13,147 \\
\text { Amaldi et al, } A-1,39 . \\
\text { Goloborodko, } \mathrm{G}-13,147 .\end{array}$ \\
\hline & $T h=B e$ & 0.7 & 5.8 & $\begin{array}{l}\text { Coloborodko, a-13, 47, Seo } \\
\text { reference sheets ror discussion. }\end{array}$ \\
\hline & $D . D$ & $2.38-2.5$ & 3.39 & soks and cukuchs, $1-2,39$. \\
\hline
\end{tabular}
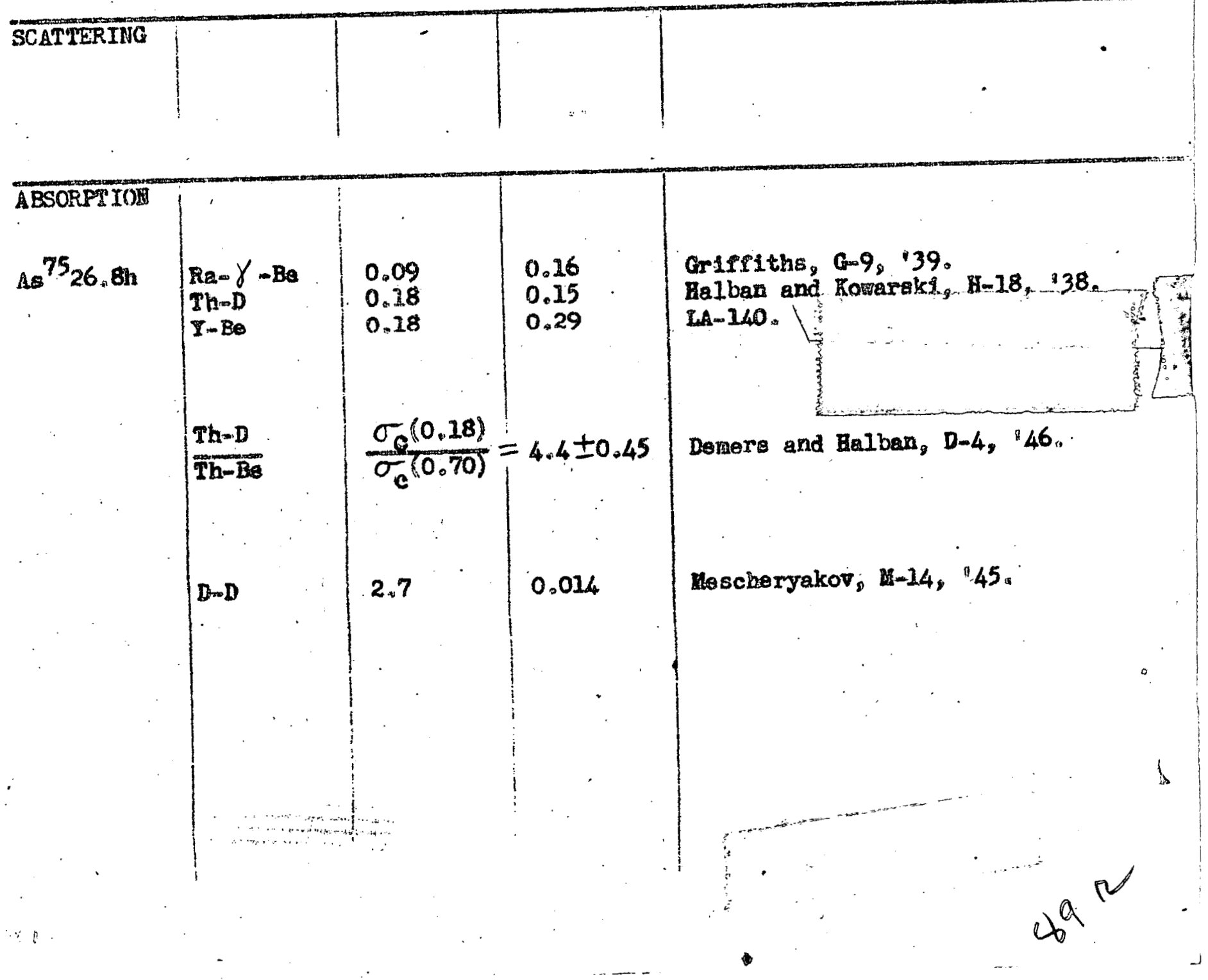


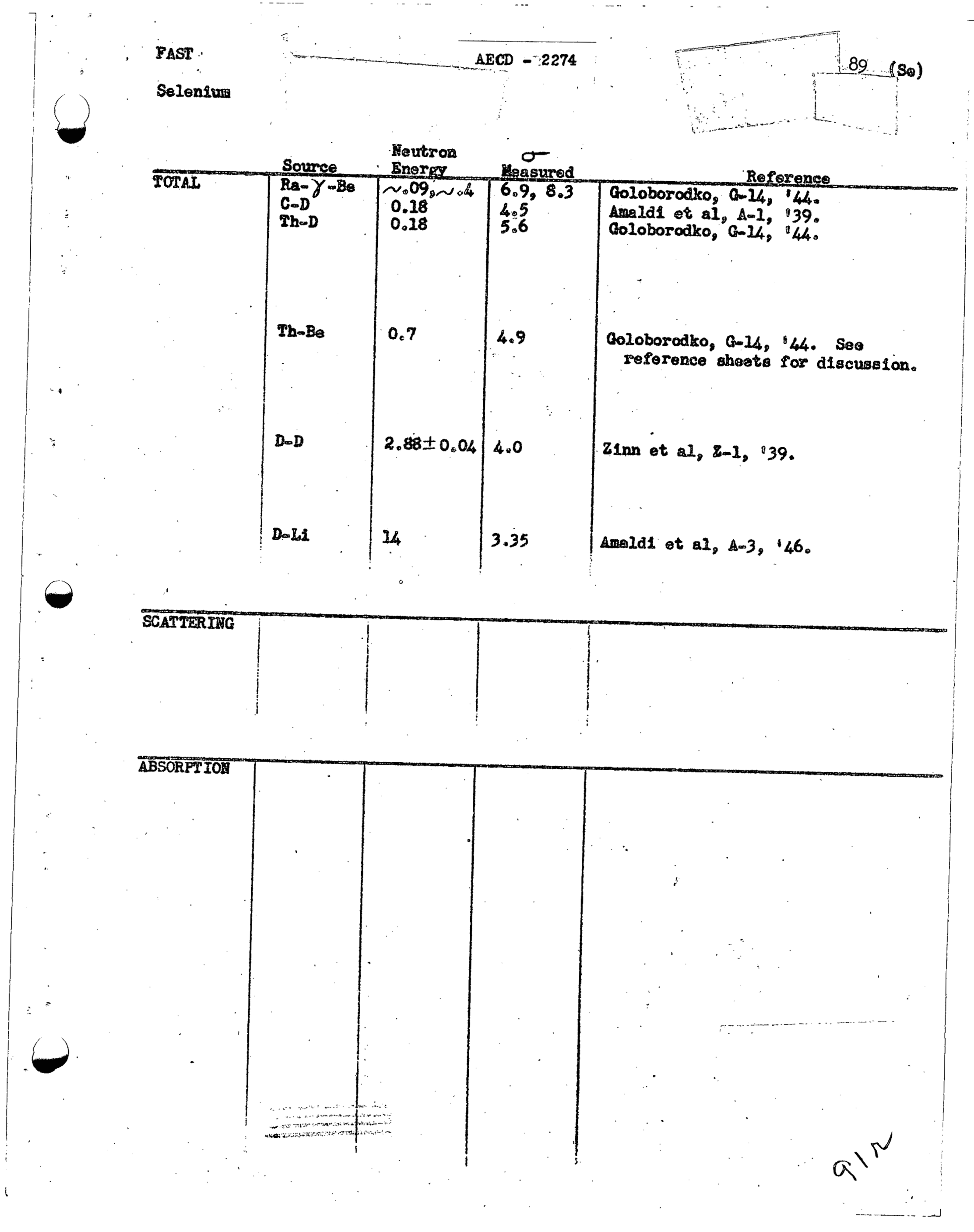




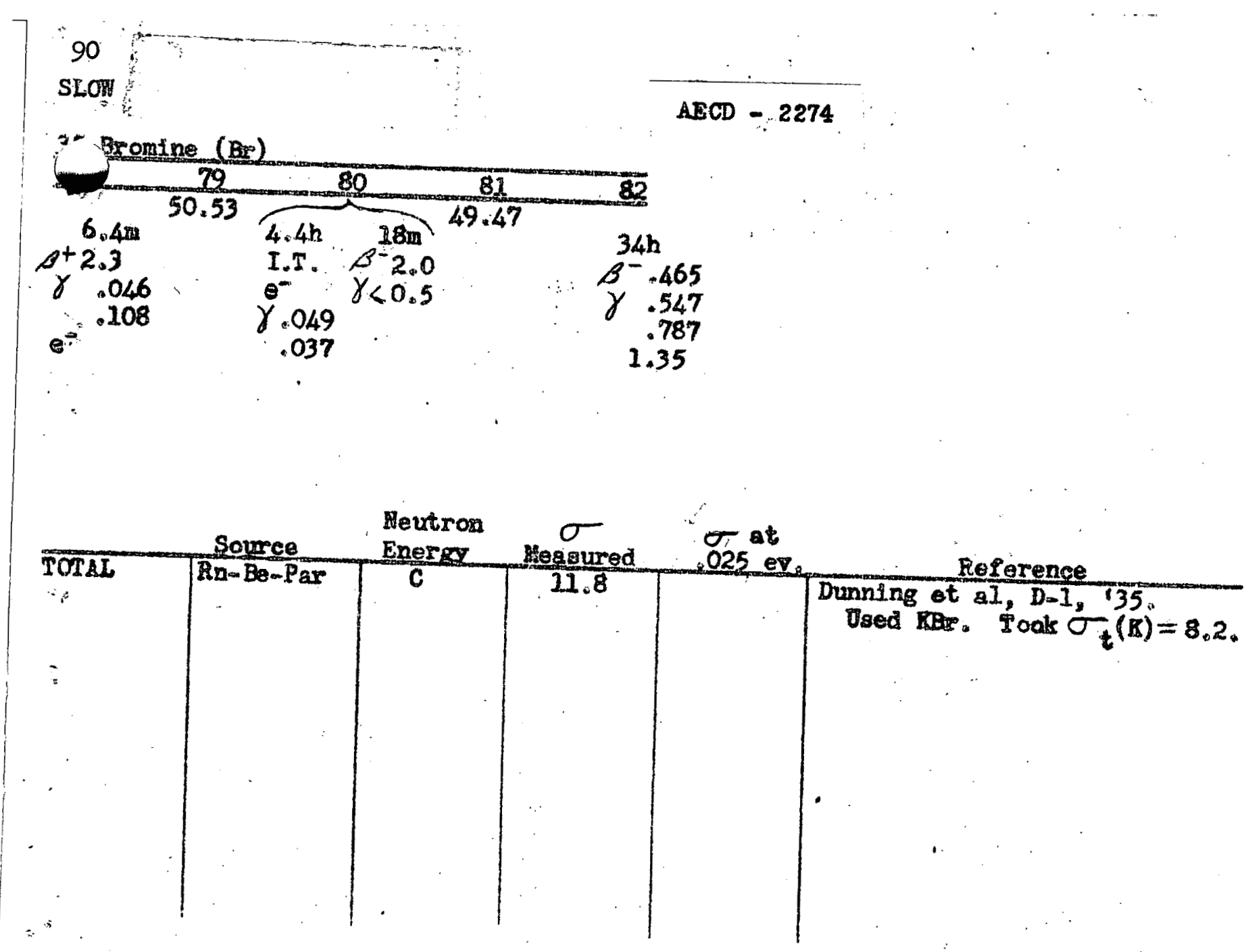

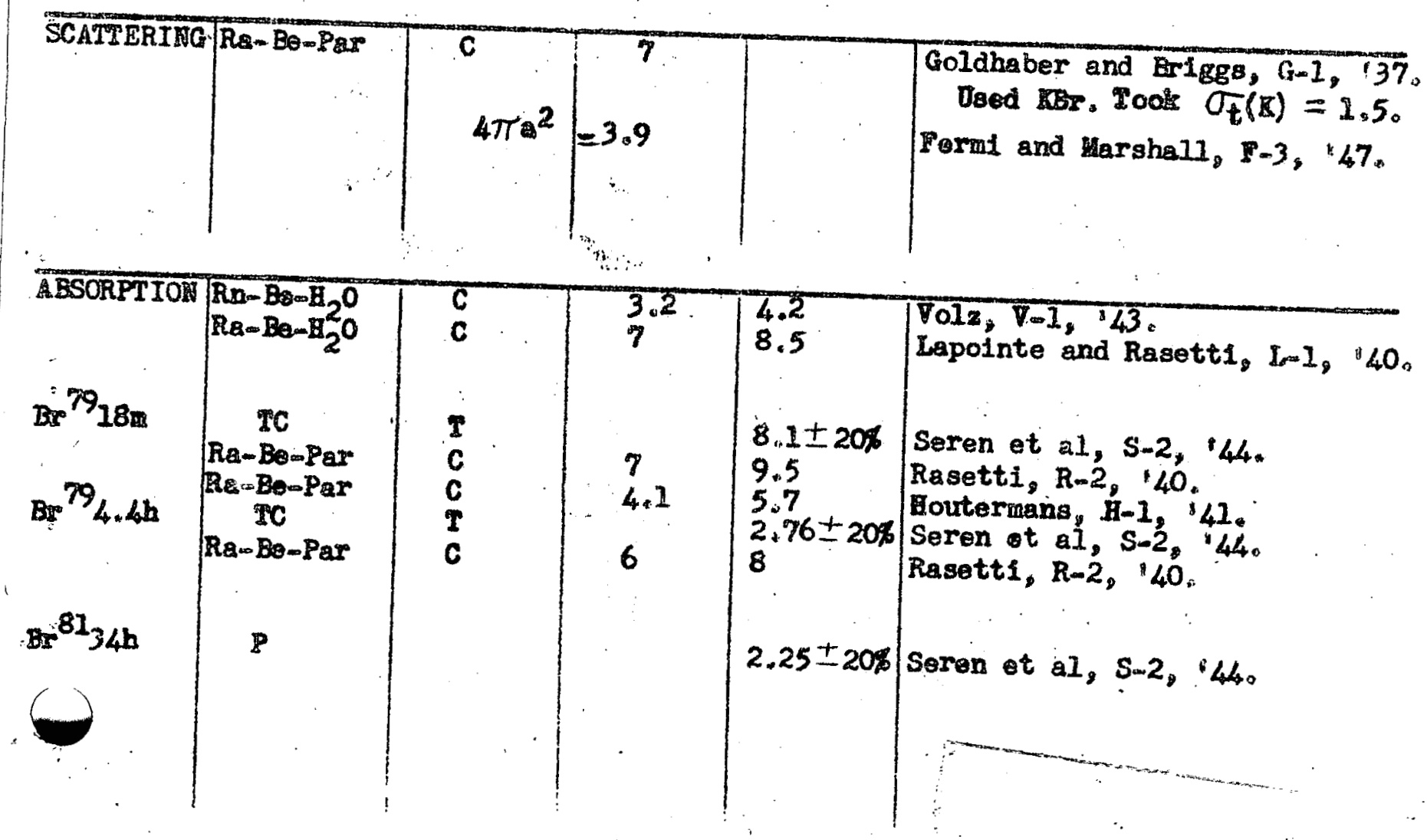




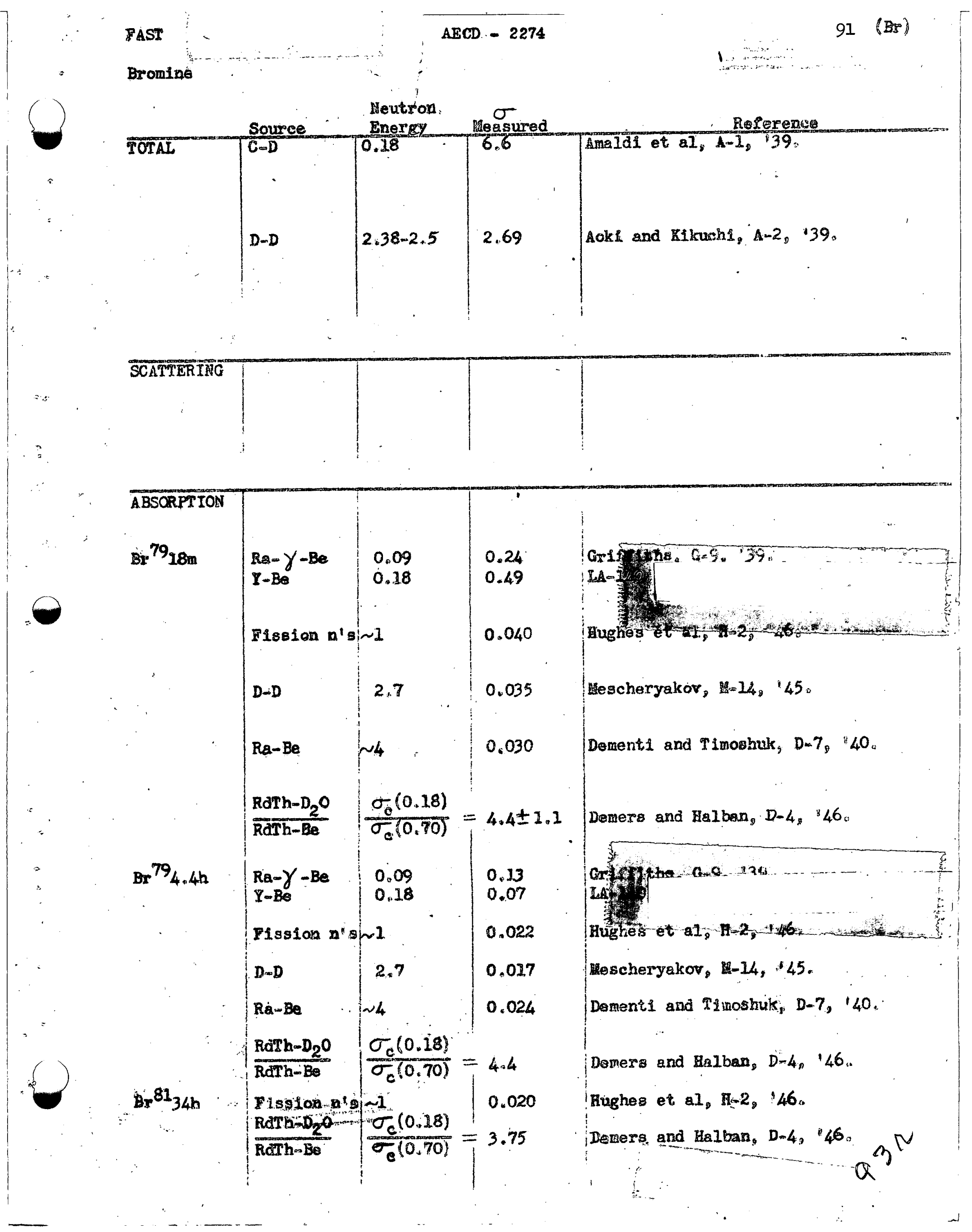



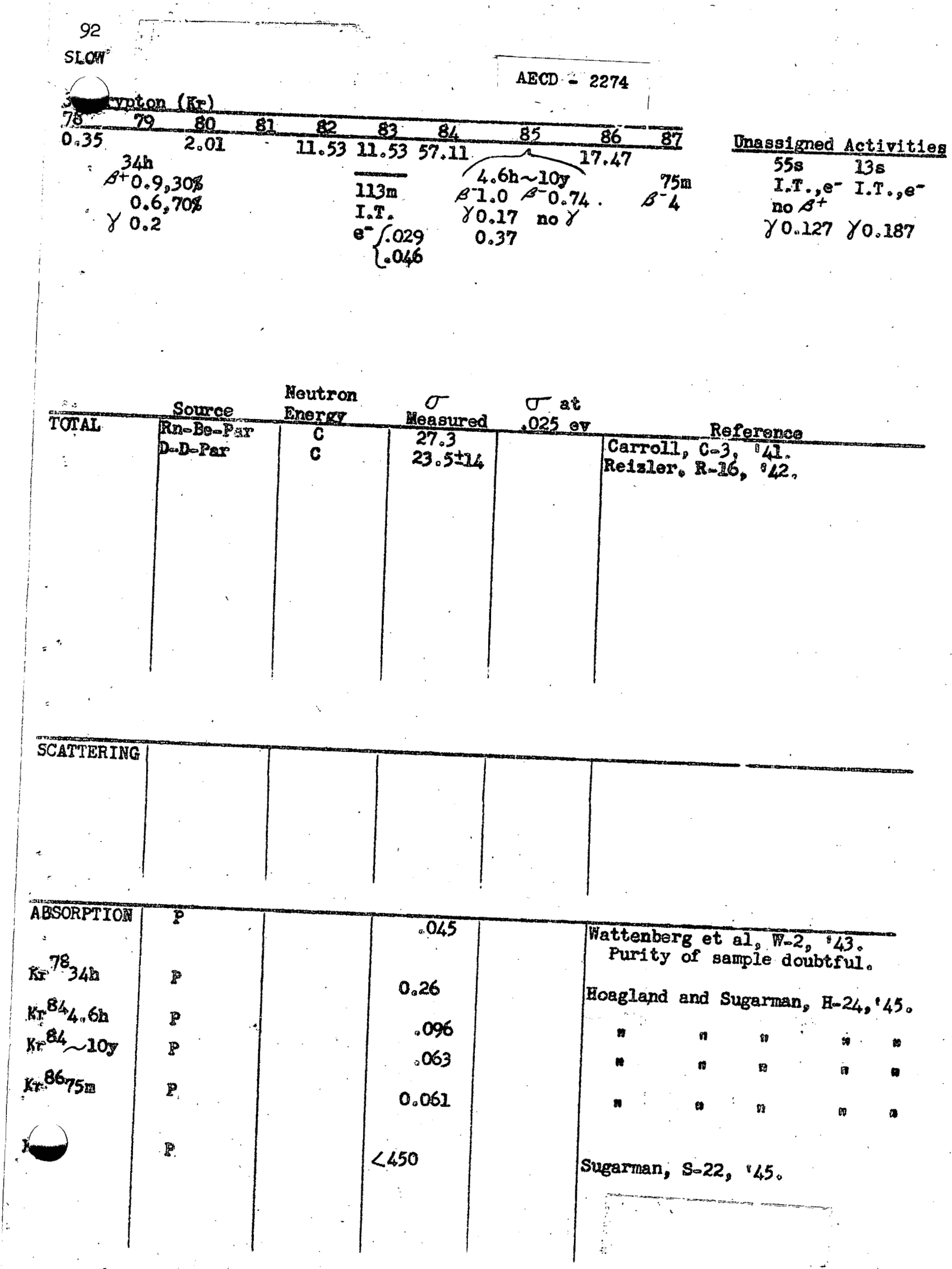
94

SION,

abfinger (Rb)

$\frac{8}{85}$

$\frac{86-\frac{87}{272}-88}{8}$

6.52

$19.5 \mathrm{~d} 5.8 \times 1010$

$B 7.6 \quad B^{-13}: \mathcal{B}^{-17.56}$

$\mathrm{e}^{-} .034$

.053

.082

.102

.129
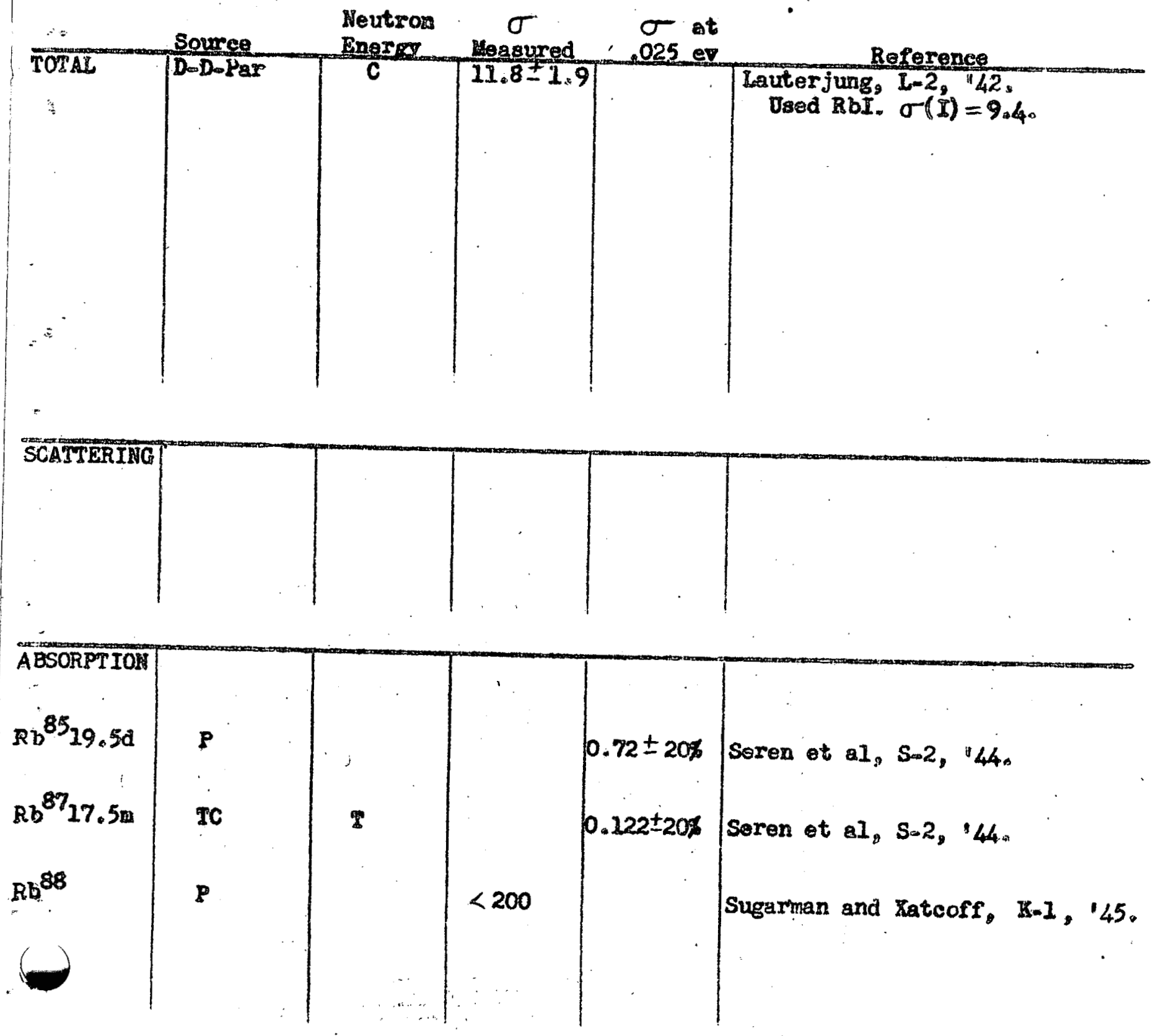

$a^{6}$ 
7 Strontium (SP)

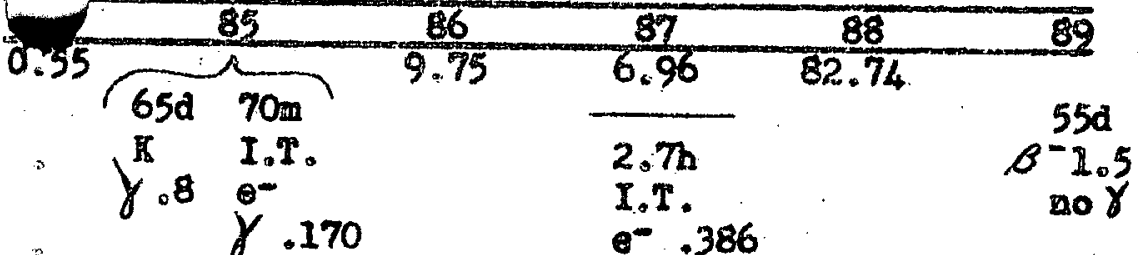

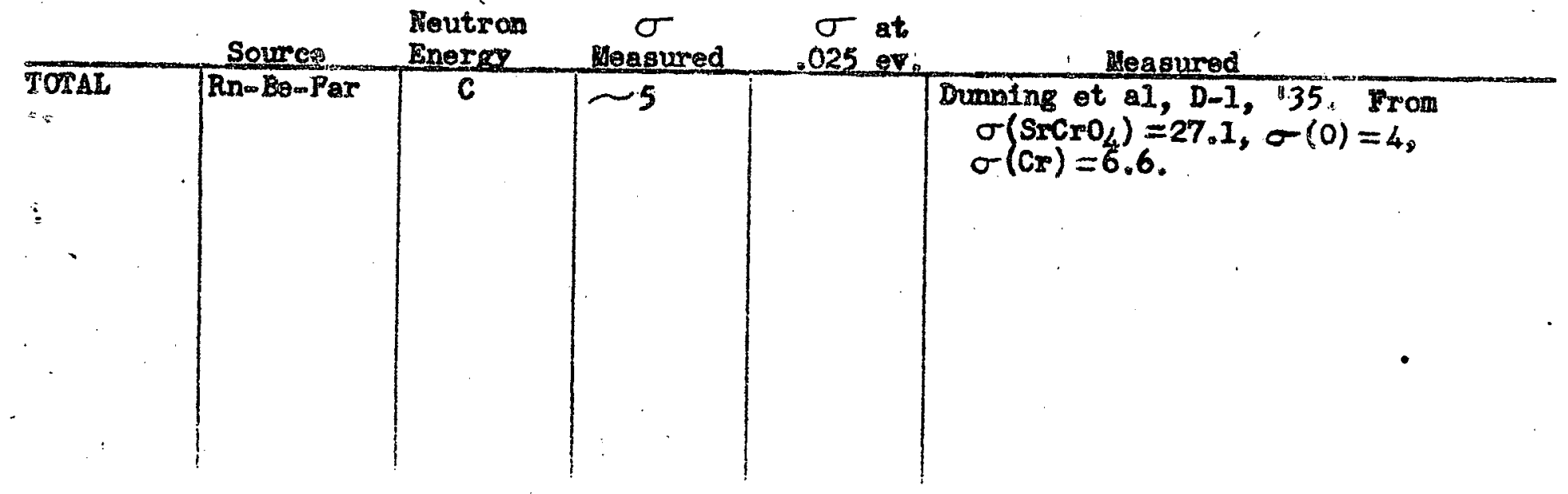

\begin{tabular}{|c|c|c|c|c|}
\hline SCATIERING & Ra $-\mathrm{Be}-\mathrm{Par}$ & C & 9.5 & $\begin{array}{l}\text { Coldhaber and Briggs, } 6-1,137 \\
\text { Jsed SrS, } \sigma_{s}(\mathrm{~s})=1.1\end{array}$ \\
\hline
\end{tabular}

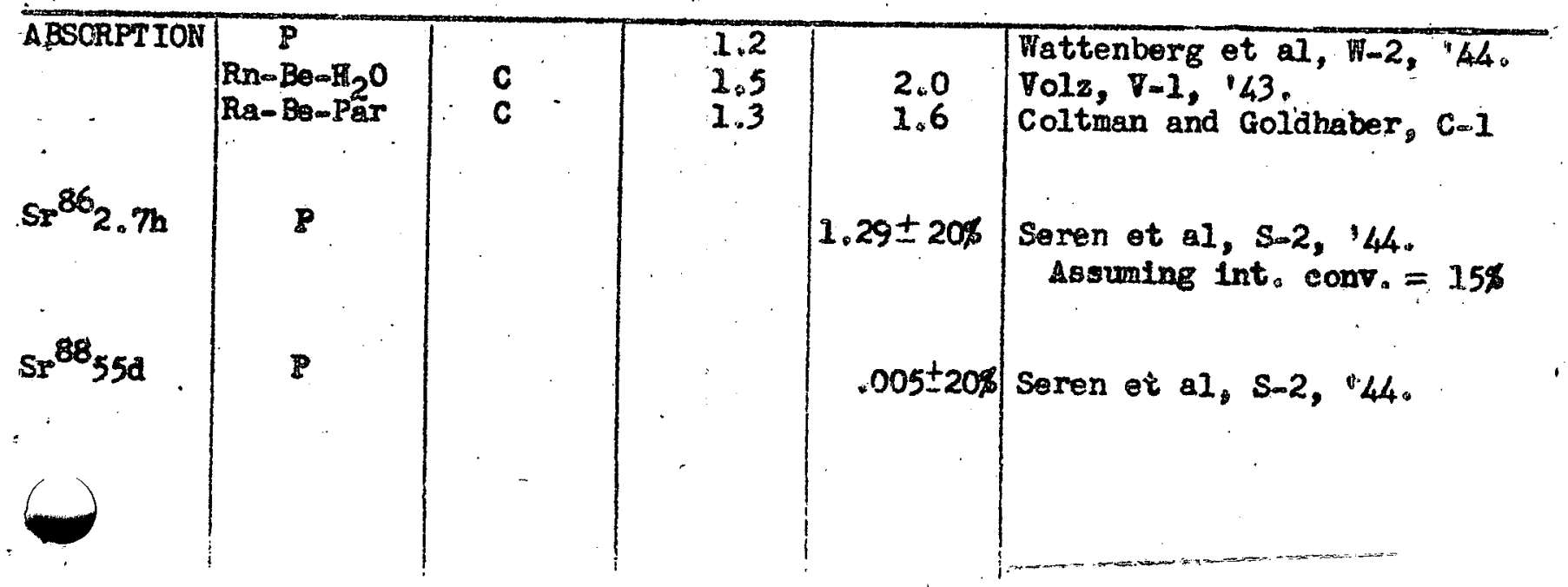


Ittrium
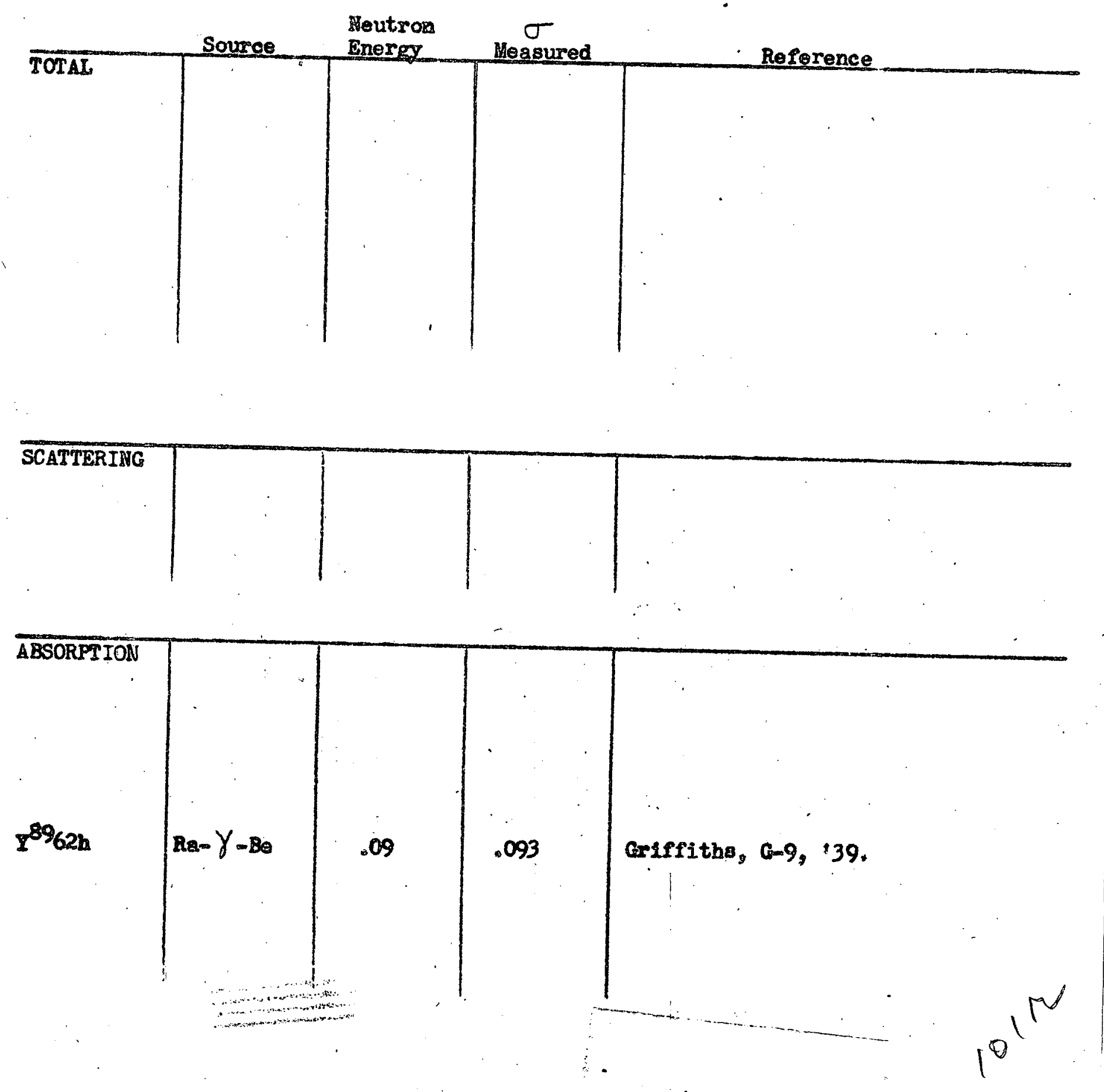

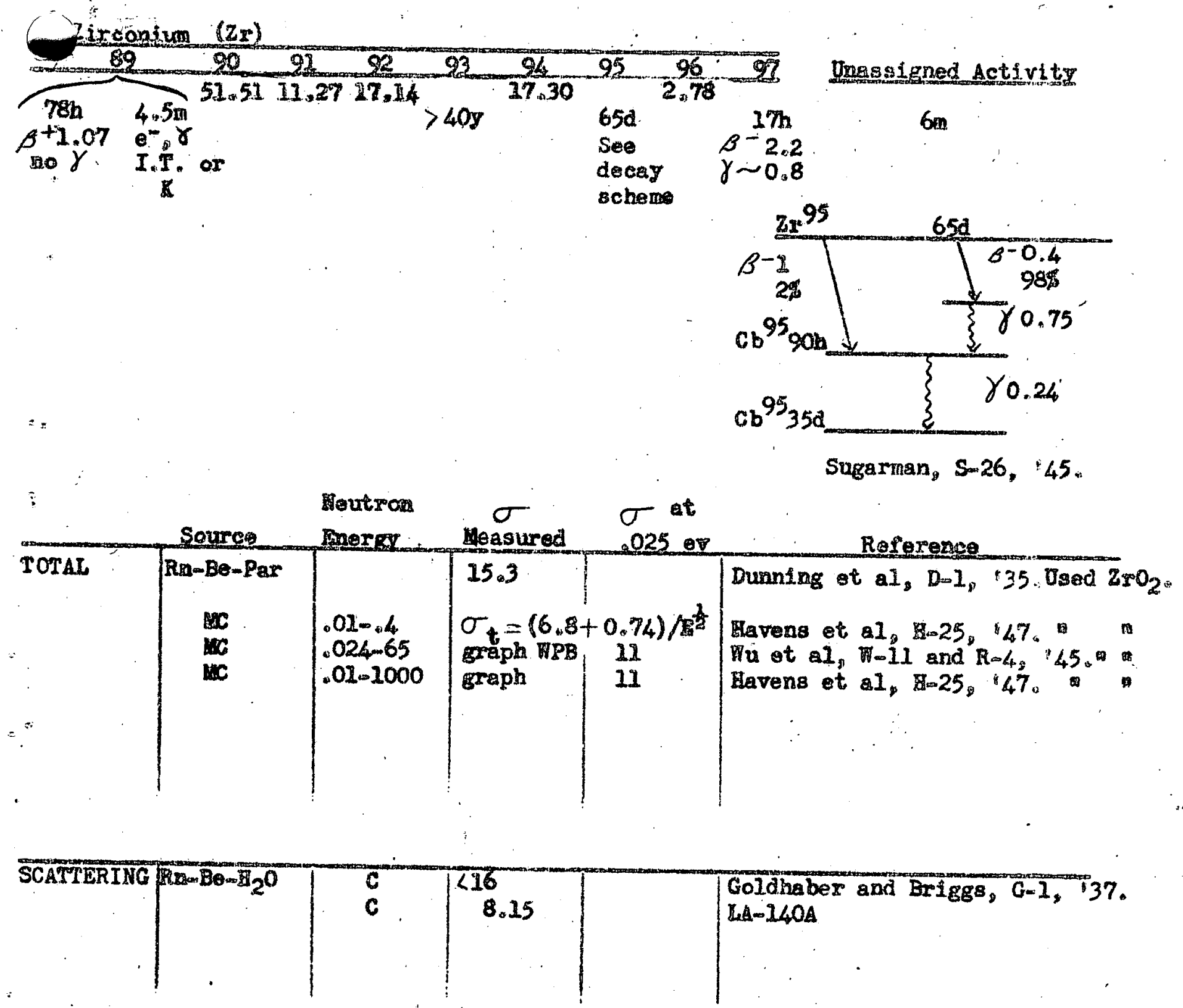

\begin{tabular}{|c|c|c|c|c|c|}
\hline \multicolumn{2}{|c|}{ ABSORPT IOR $R \mathrm{Rn}-\mathrm{BO}-\mathrm{H}, 0$} & \multirow{5}{*}{ c } & \multirow[b]{2}{*}{$\begin{array}{c}<0.35 \\
2.8\end{array}$} & \multirow{3}{*}{$\begin{array}{r}<0.4 \\
2.0 \\
1.1 \\
\\
0.43 \pm 408\end{array}$} & \multirow{3}{*}{ 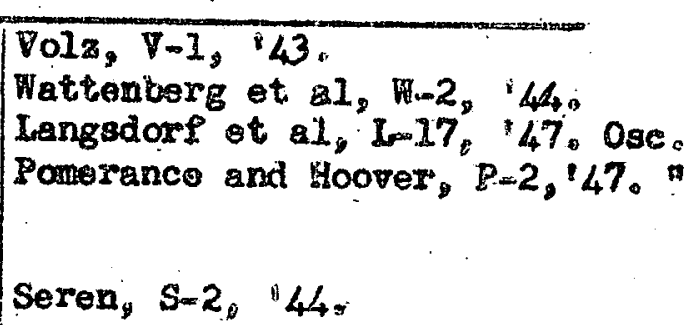 } \\
\hline ABSORPT TOEY & $\begin{array}{c}\mathrm{R} n-\mathrm{BO}-\mathrm{H}_{2} \mathrm{O} \\
\mathrm{P} \\
P \\
P\end{array}$ & & & & \\
\hline $2 x 9465 d$ & $\mathbb{P}$ & & & & \\
\hline $20 x^{96} 17 \mathrm{~h}$ & $P$ & & & $0.60 \pm 20 \%$ & -2 \\
\hline & TC & & & $\begin{array}{l}\text { atomis } \\
.016 \pm 40 \%\end{array}$ & $\approx \quad \therefore$ \\
\hline
\end{tabular}




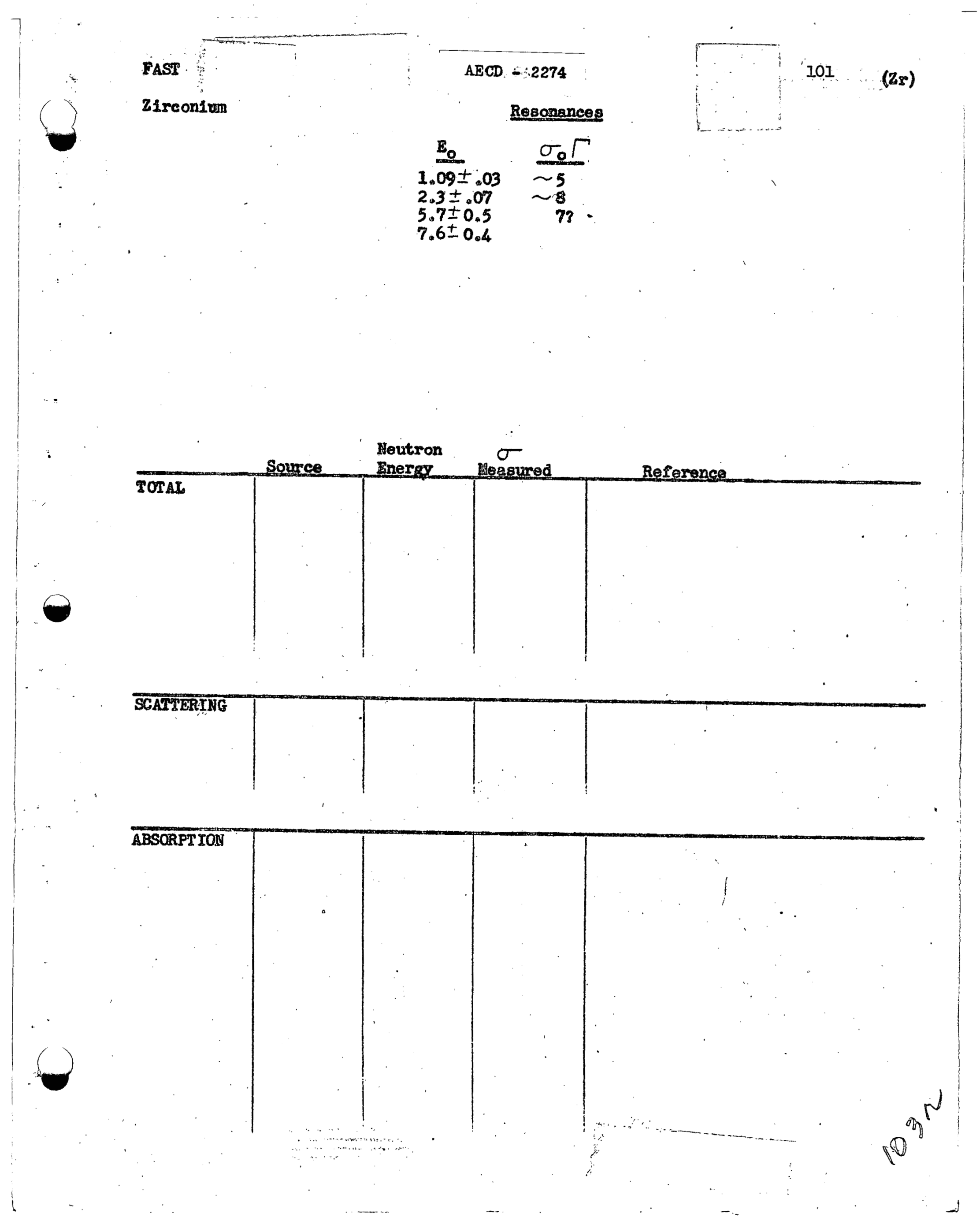




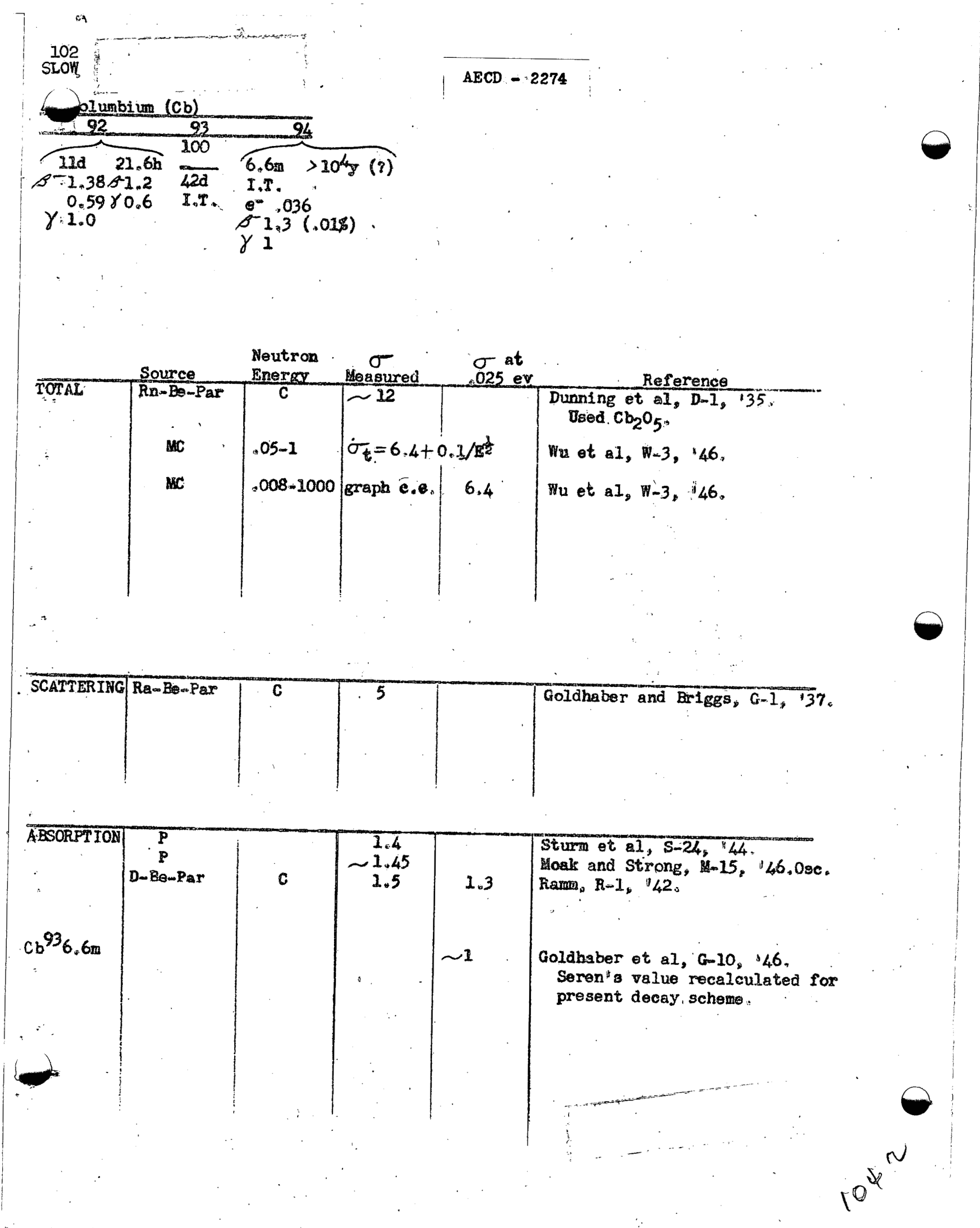




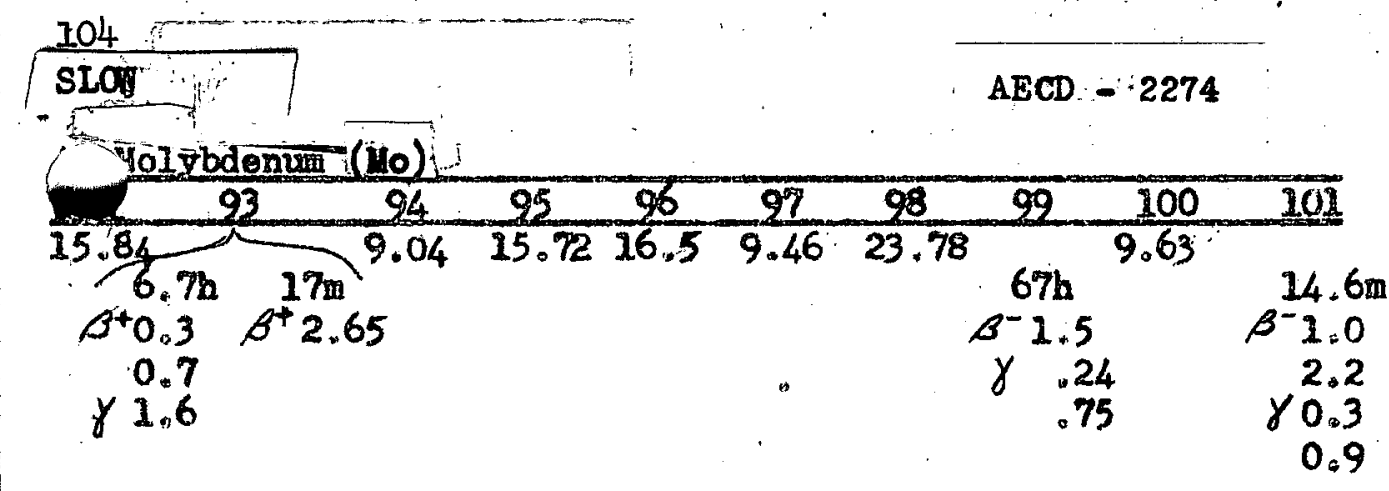

\begin{tabular}{|c|c|c|c|c|c|}
\hline$\cdots$ & Source & $\begin{array}{l}\text { Houtron } \\
\text { Enerey }\end{array}$ & $\underset{\text { Measured }}{\sigma}$ & $\sigma$ at & Reforenge \\
\hline TOTAL & $R n=B o-P a x$ & $\mathrm{c}$ & 7.1 & & Dunning ot al, Del, 135 \\
\hline $\mathrm{MOO}_{3}$ & Ra-y-Be-Par & c & 18.5 & & Demers, $D-2,46$. \\
\hline $\mathrm{HoO}_{3}$ & Ra- $Y_{-B}-\mathrm{Be}$ Par & AuRes 4.8 & .16 .1 & & Demers, D-2, 146 . \\
\hline
\end{tabular}

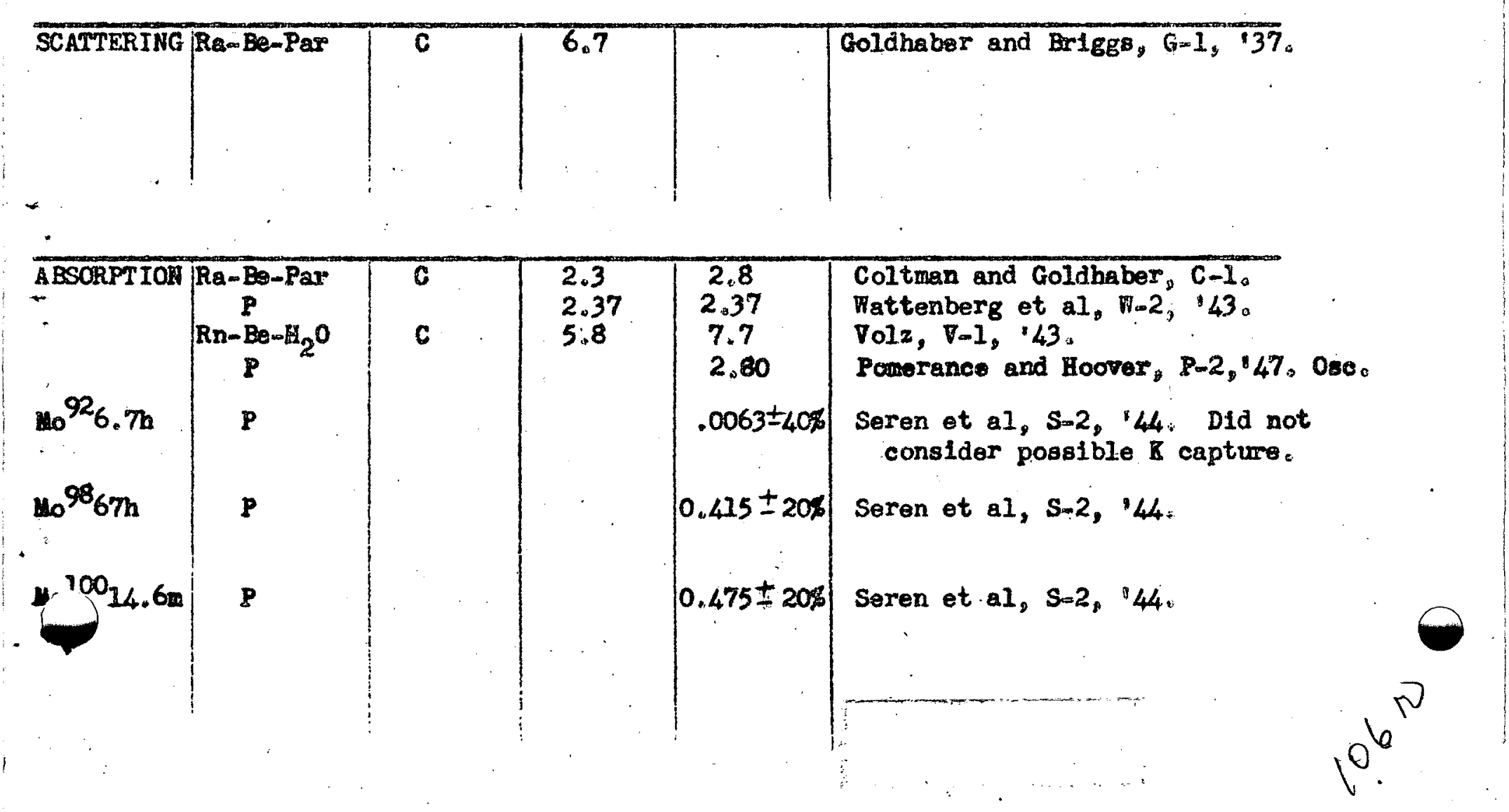



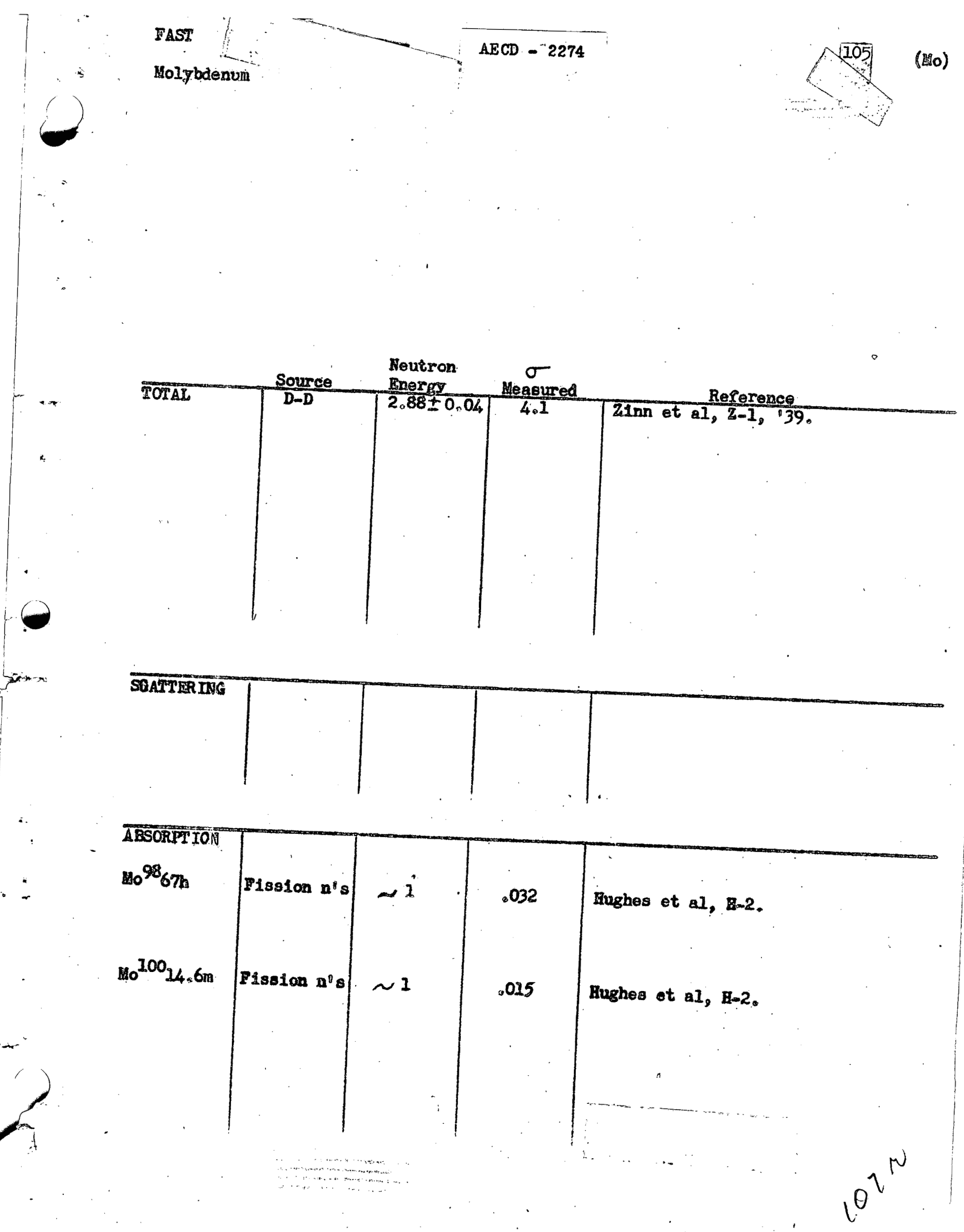
(5ehnetiun (To)

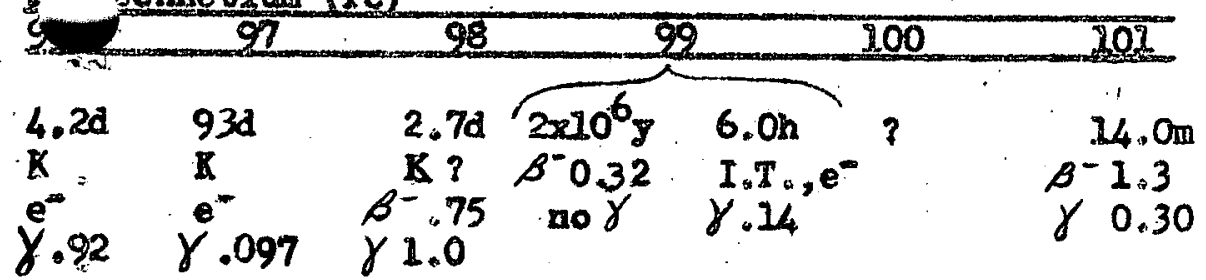

$2,7 \AA$

3

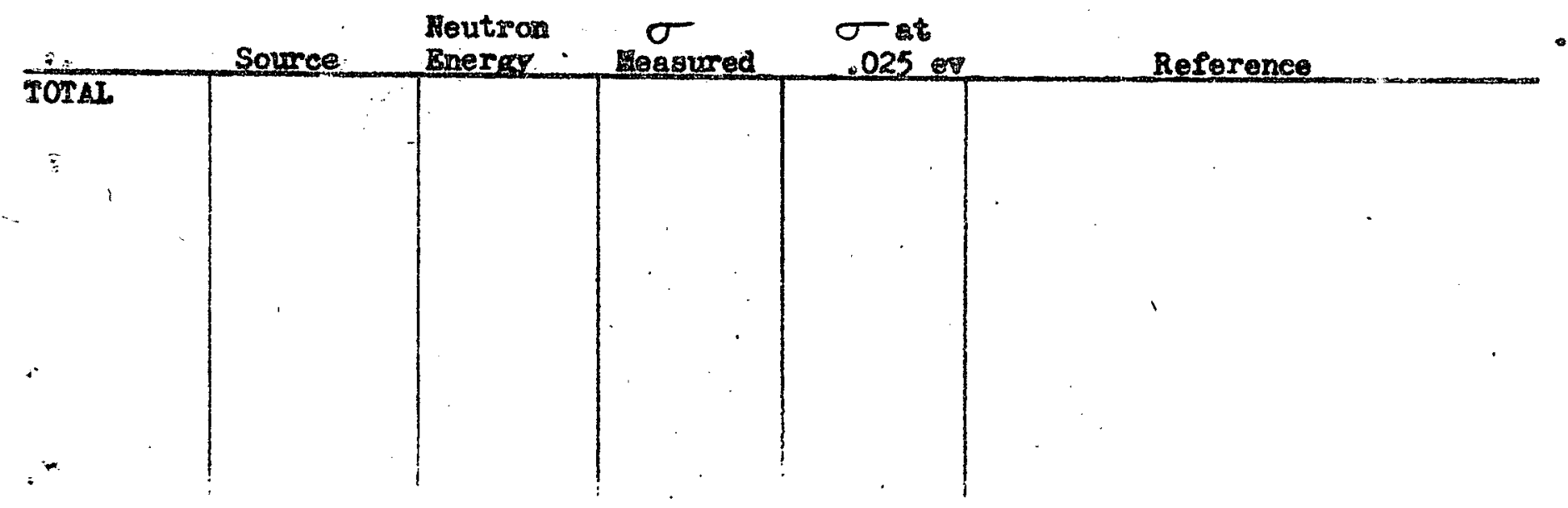

$a$
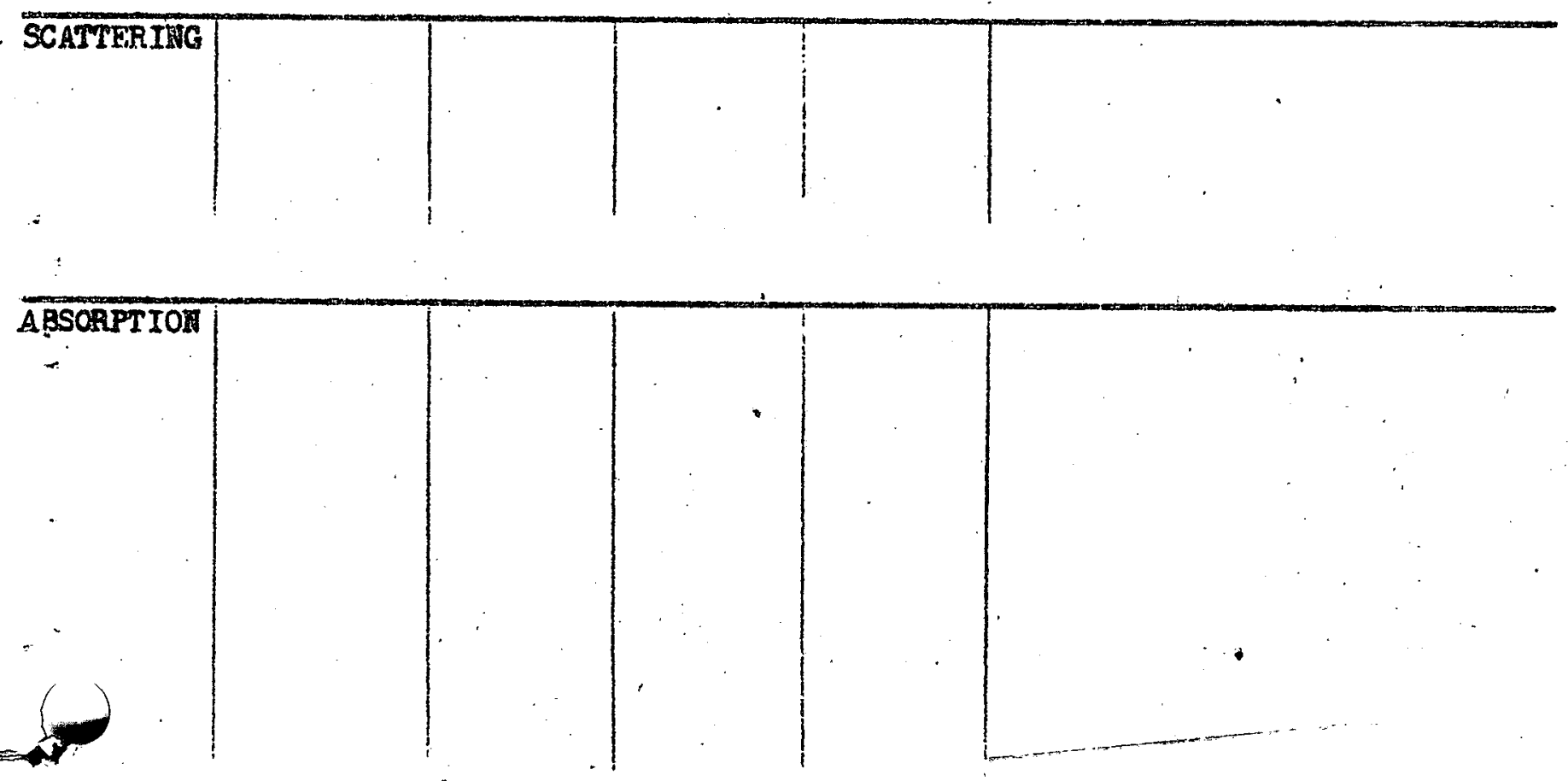
6 uthenium (Ru)

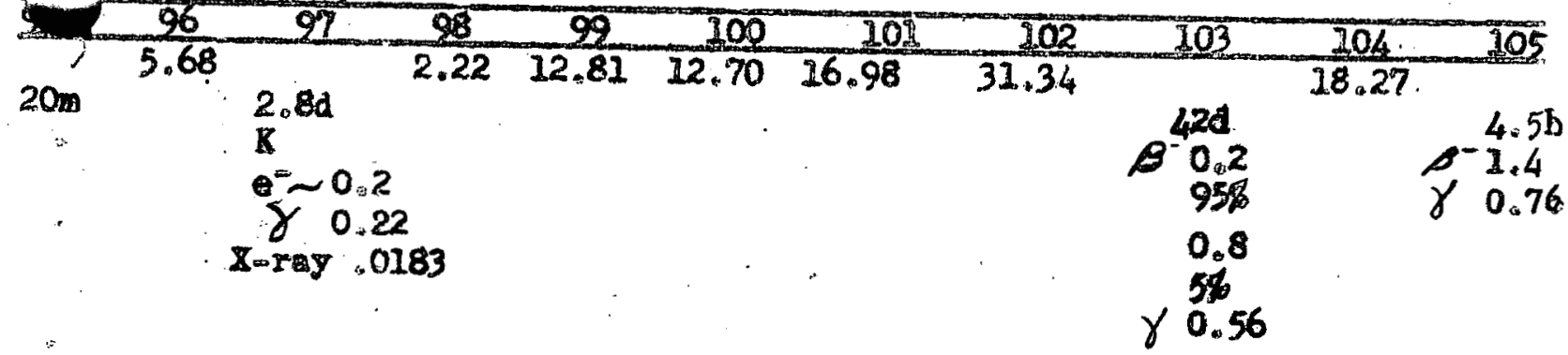
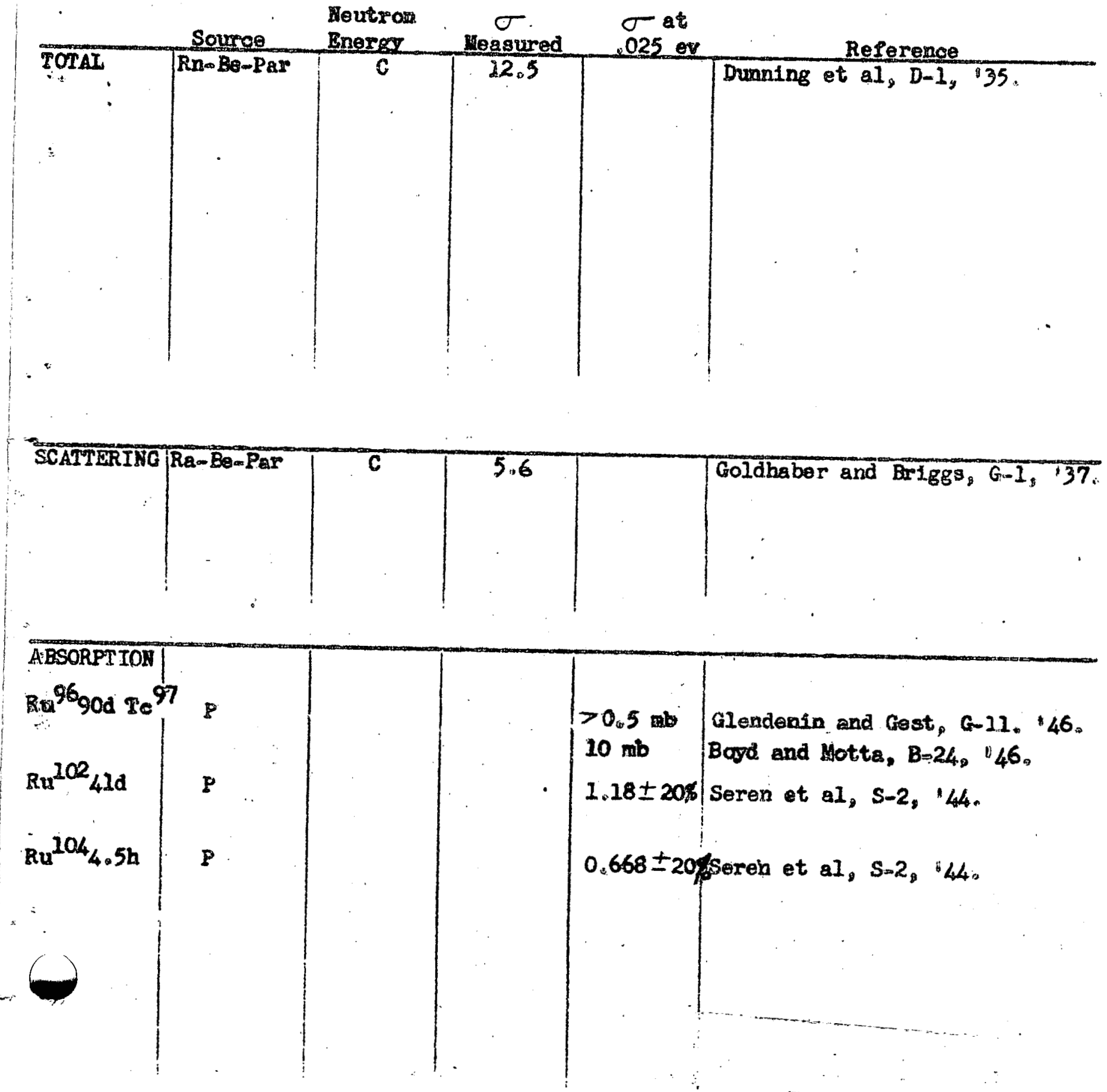


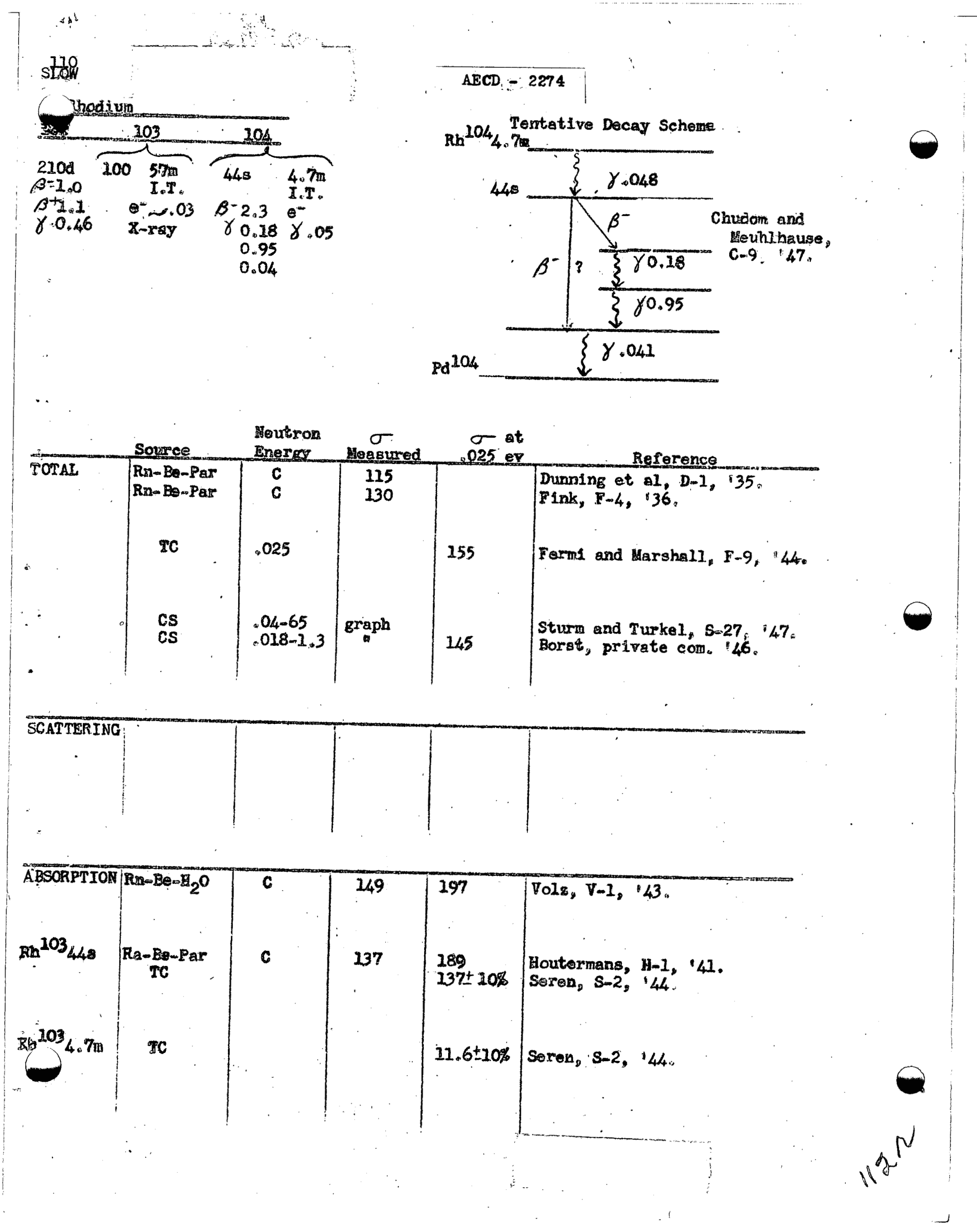




$$
\underset{1.29}{\text { so }_{8}} \frac{\Gamma}{\Gamma}<\frac{\sigma_{0}}{\Gamma}
$$
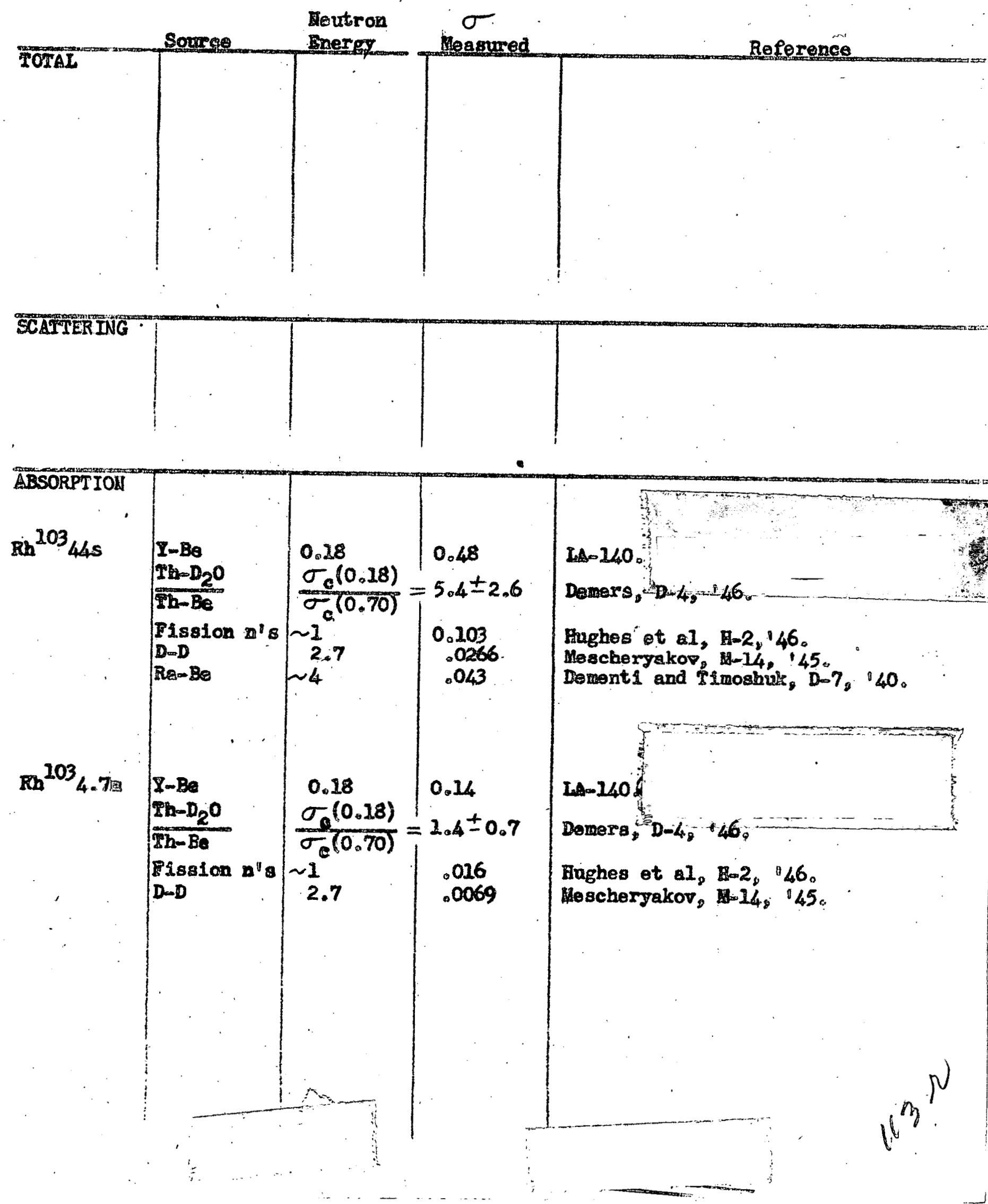

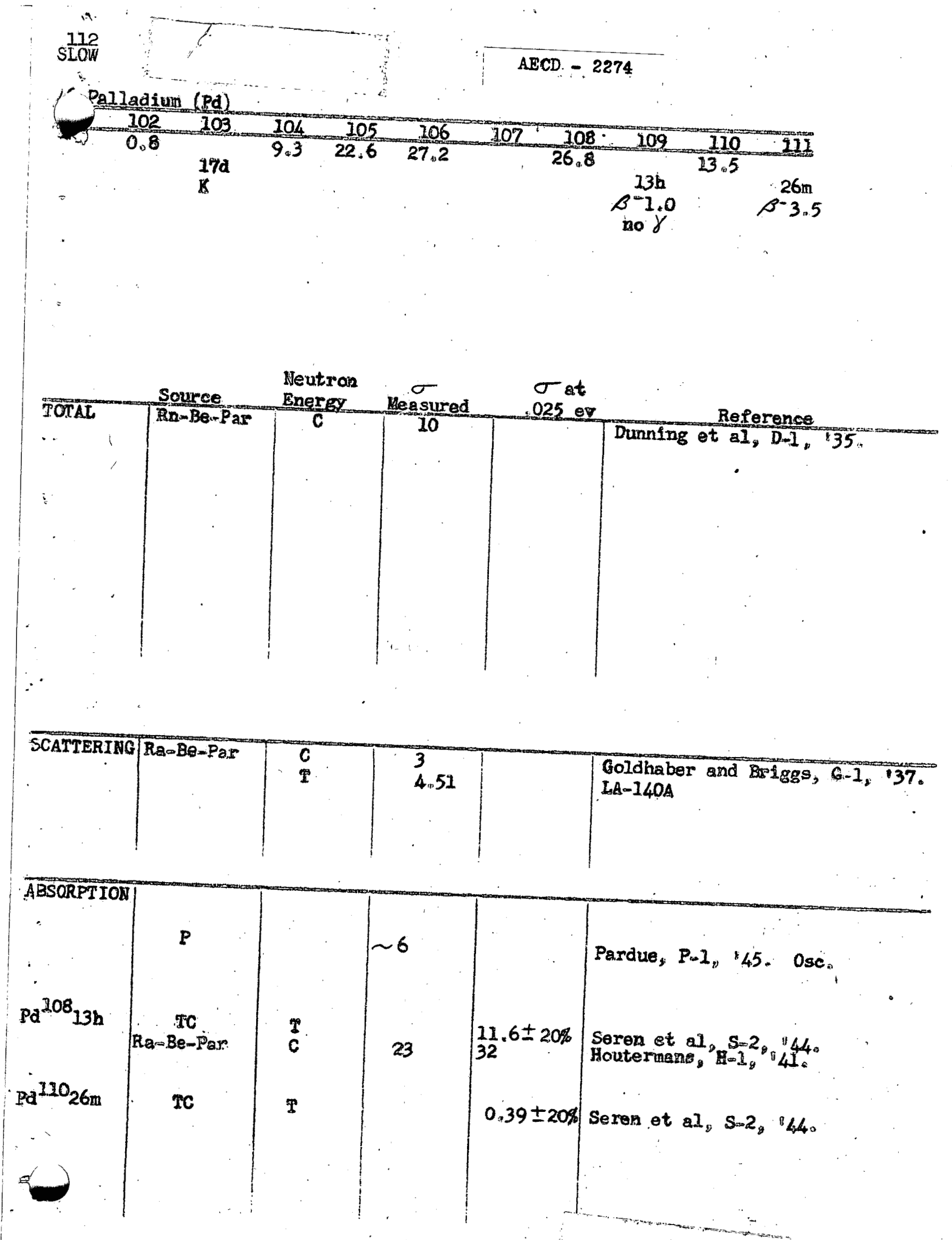

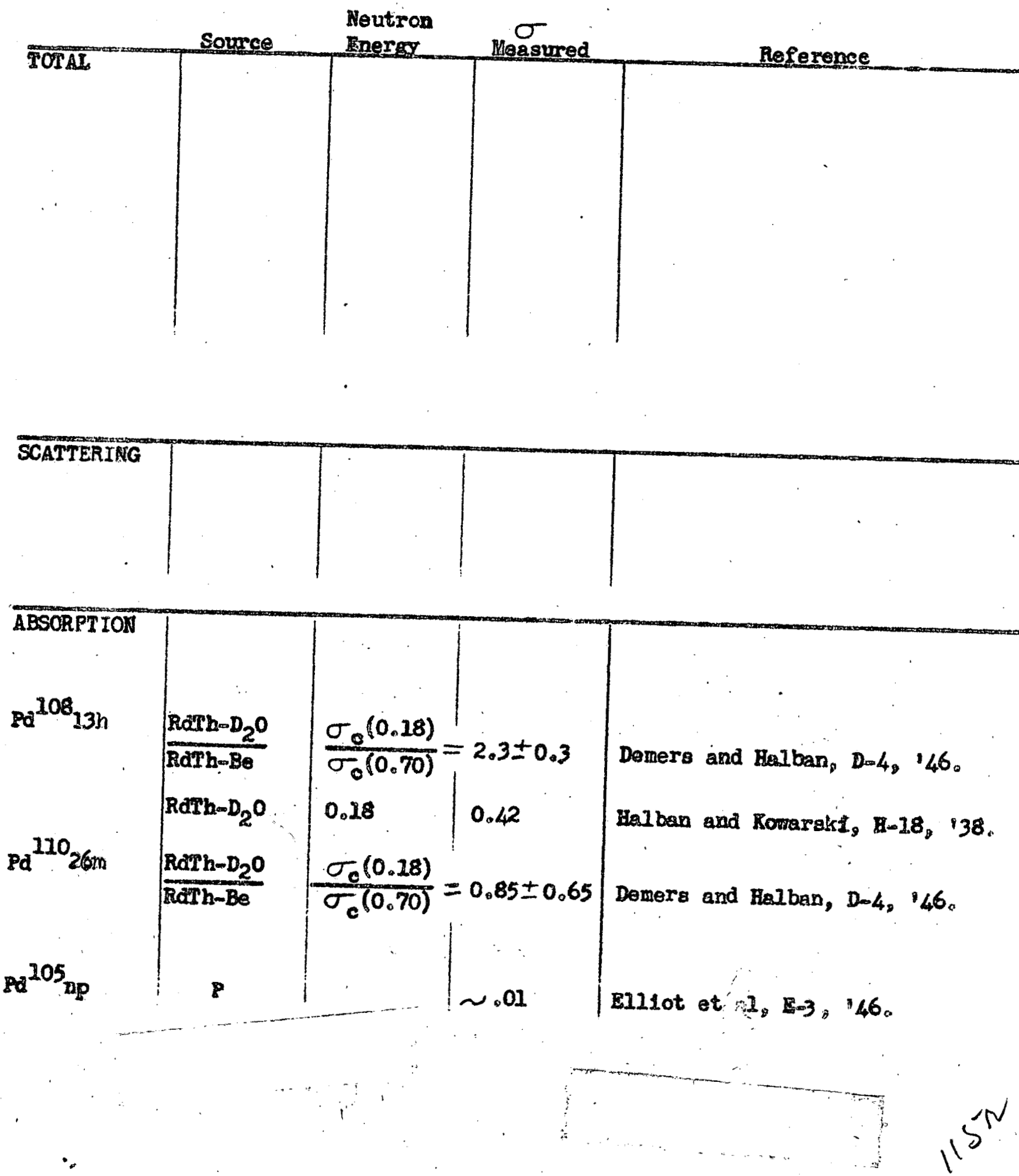


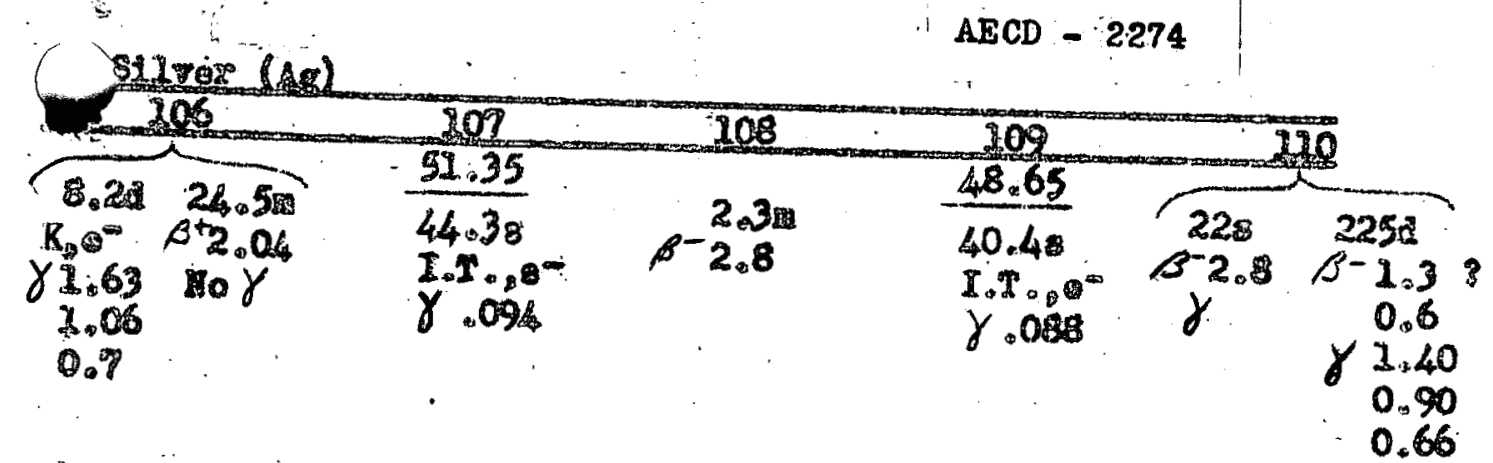

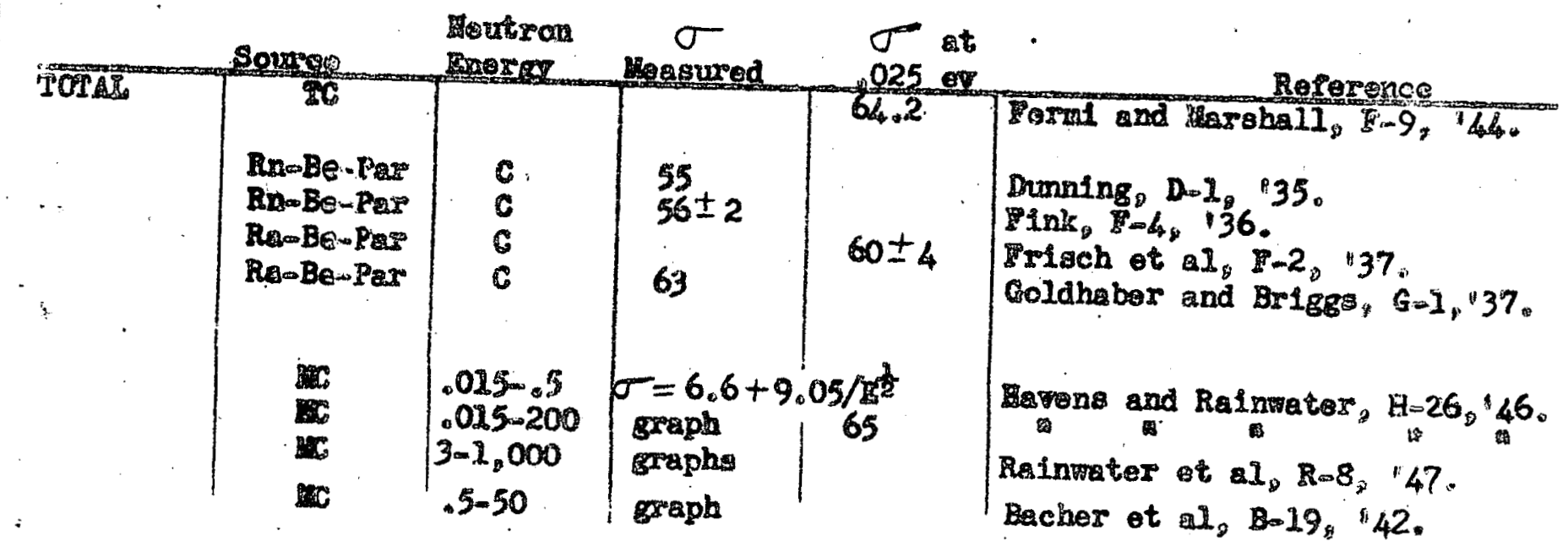

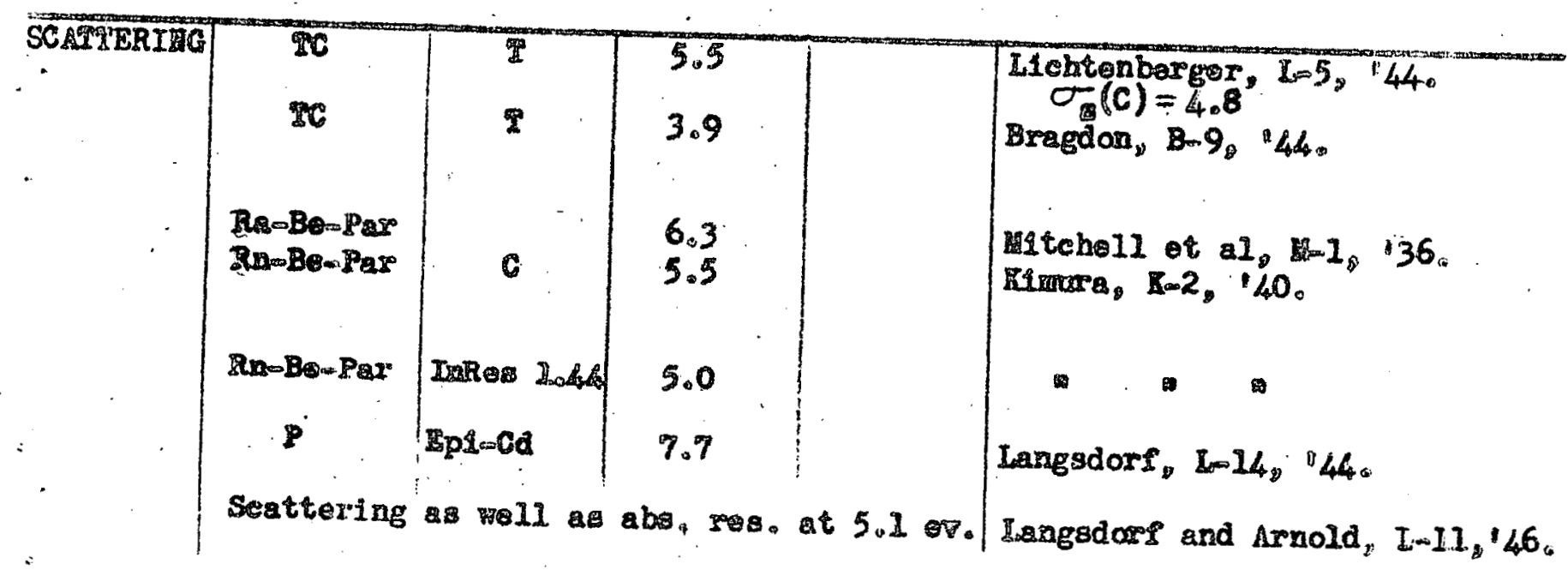

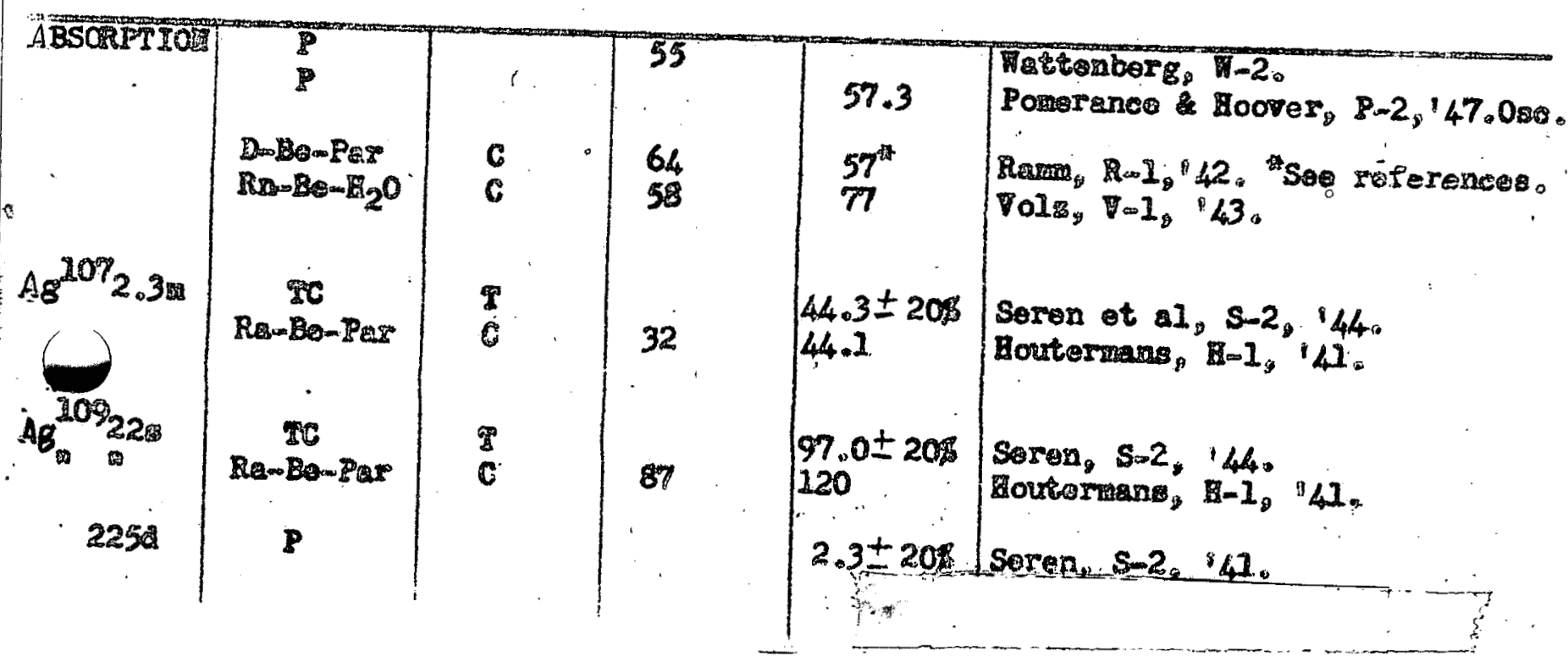




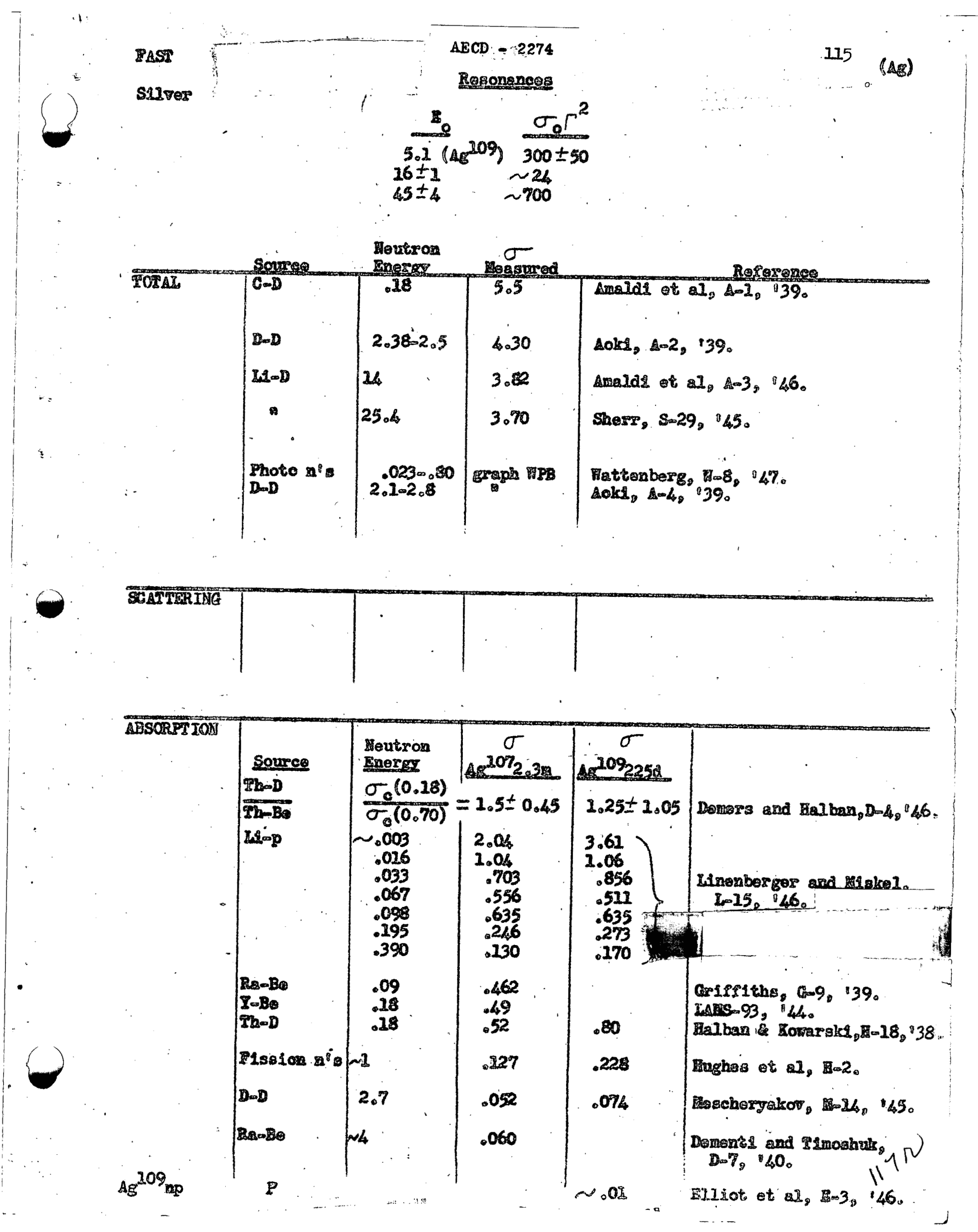




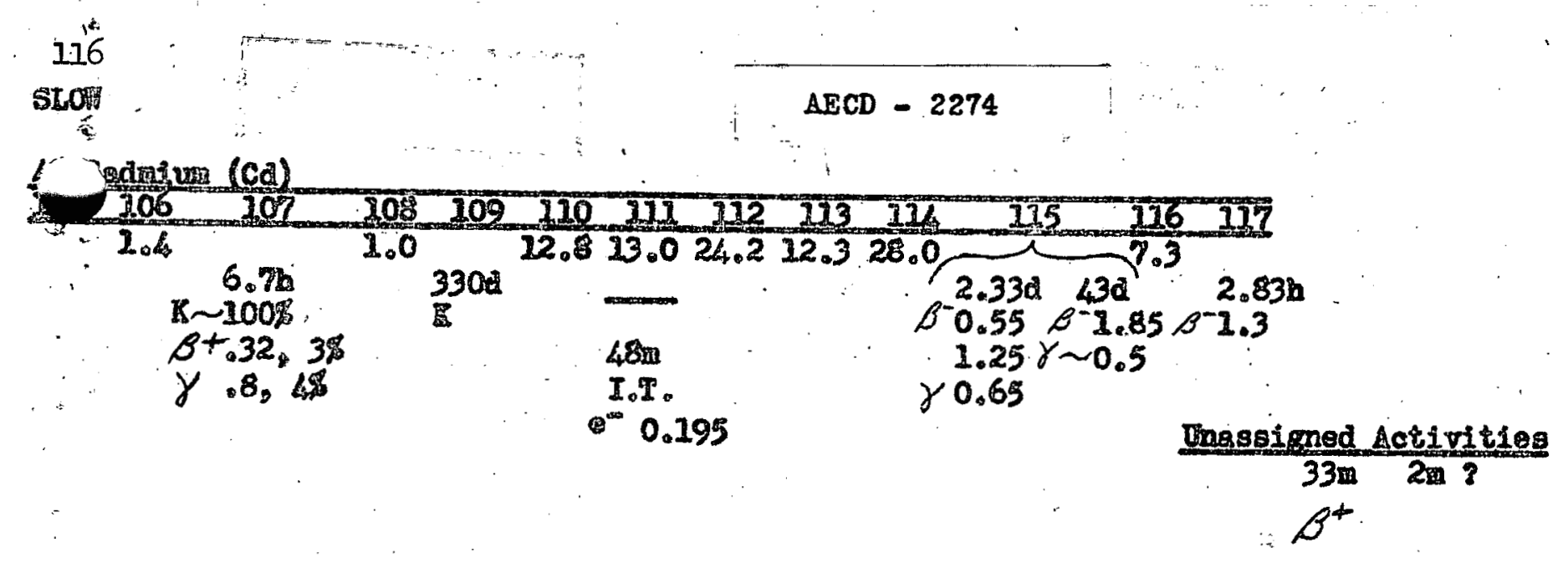

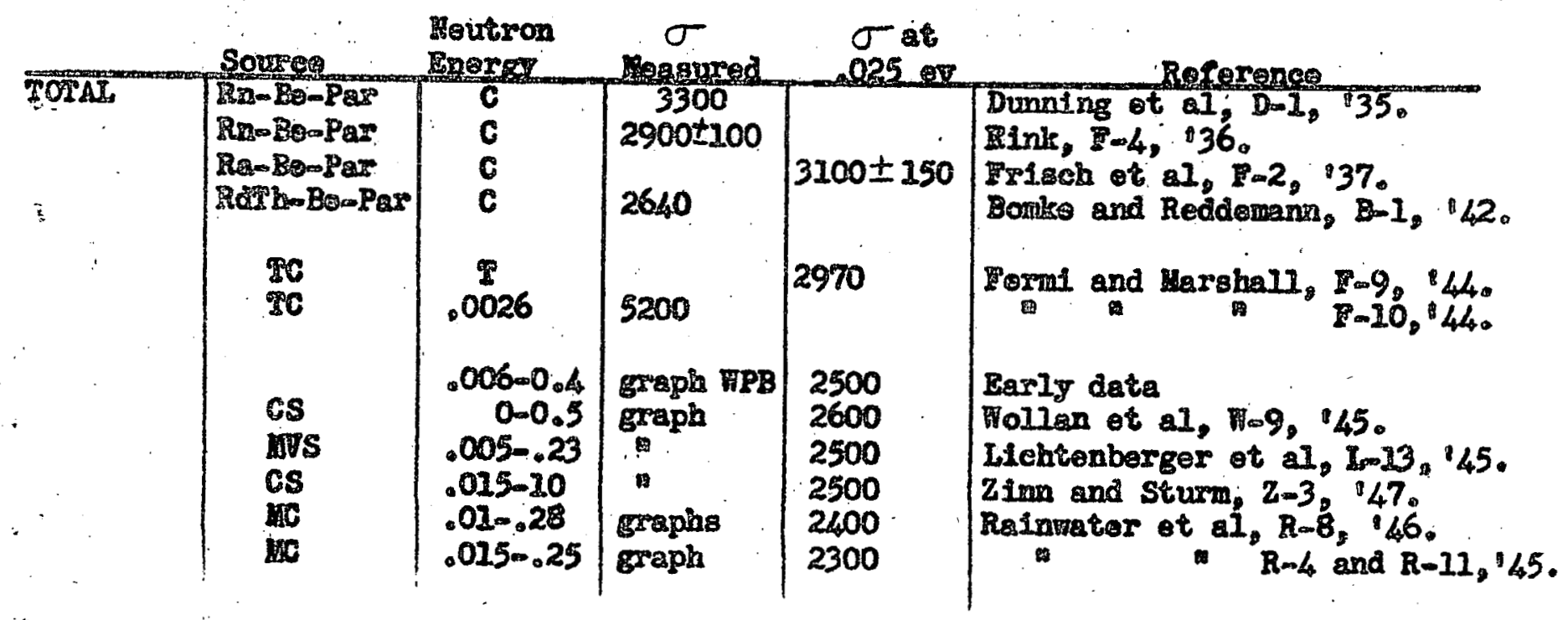

\begin{tabular}{|c|c|c|c|c|}
\hline SCATTERIRG & $\begin{array}{c}\text { Re-Bospr } \\
\text { TC } \\
\text { TC }\end{array}$ & $\begin{array}{c}\frac{9}{3} \\
.01-2 \\
.01-10\end{array}$ & $\begin{array}{l}1.2 \\
6 \pm 1 \\
7.5 \\
\begin{array}{l}\text { Constant tern }=5.3 \\
6.0\end{array}\end{array}$ & 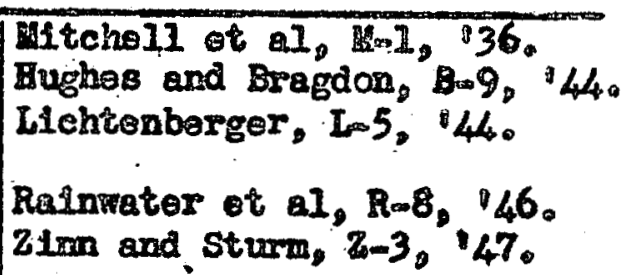 \\
\hline
\end{tabular}

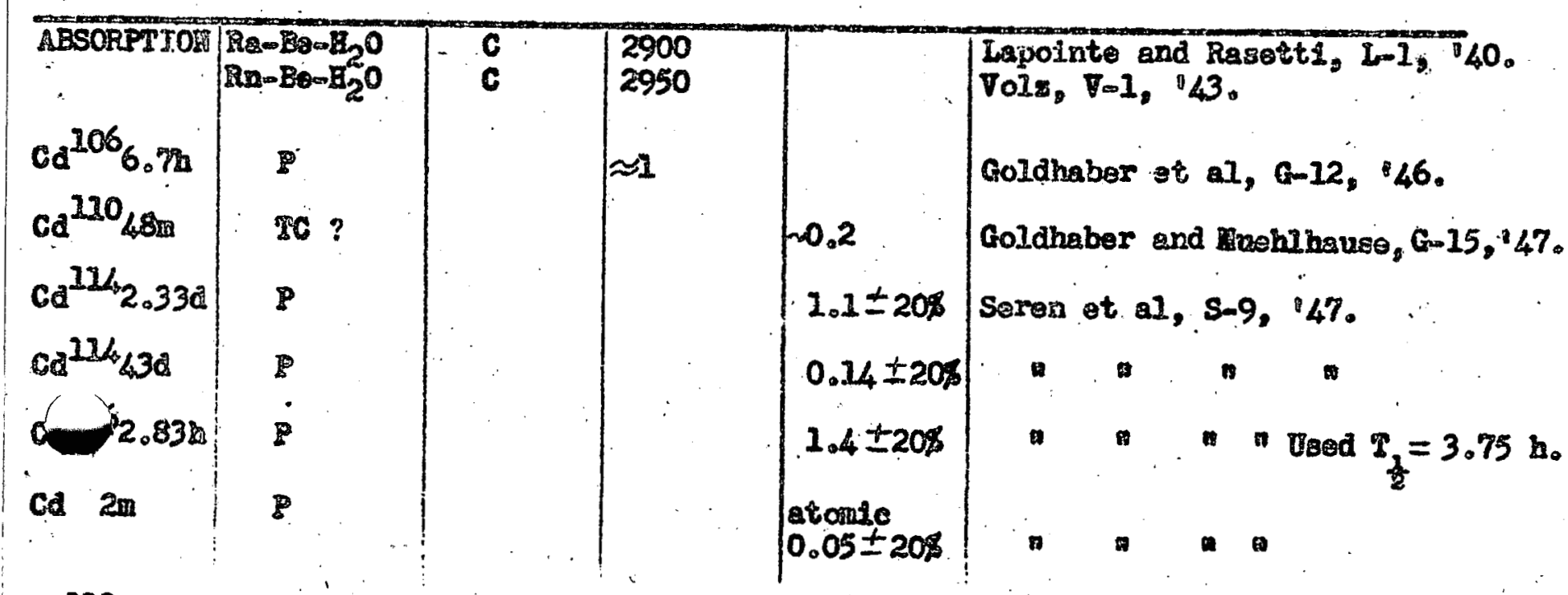

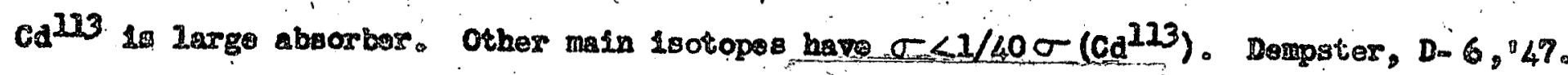


Resonancos

$$
\operatorname{cd}^{113} \stackrel{\Sigma_{0}}{0.176} \frac{\sigma_{0}}{7,200} \quad \frac{\Gamma}{0.175}
$$

\begin{tabular}{|c|c|c|c|c|}
\hline & Souree & $\begin{array}{l}\text { Eeutron } \\
\text { Energe }\end{array}$ & $\begin{array}{c}\sigma \\
\text { Regrured }\end{array}$ & Reforence \\
\hline \multirow[t]{3}{*}{ TOTAL } & $\begin{array}{l}C-D \\
D \sim D\end{array}$ & 0.18 & $\begin{array}{l}4.9 \\
4.07\end{array}$ & 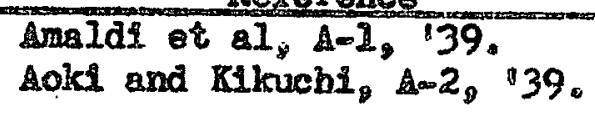 \\
\hline & Dow LI & $\mathbf{L}_{4}$ & 4.25 & Amaldi te al, $A-3, \div 46$ 。 \\
\hline & 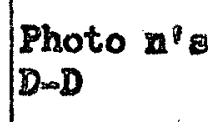 & $\begin{array}{l}.023-0.8 \\
2.2-2.8\end{array}$ & $\underset{7}{\text { graph }}$ & $\begin{array}{l}\text { Wettenberg at al, W-8. } \\
\text { Aoki, A A, 39. }\end{array}$ \\
\hline
\end{tabular}

\section{SCATIERIVG}
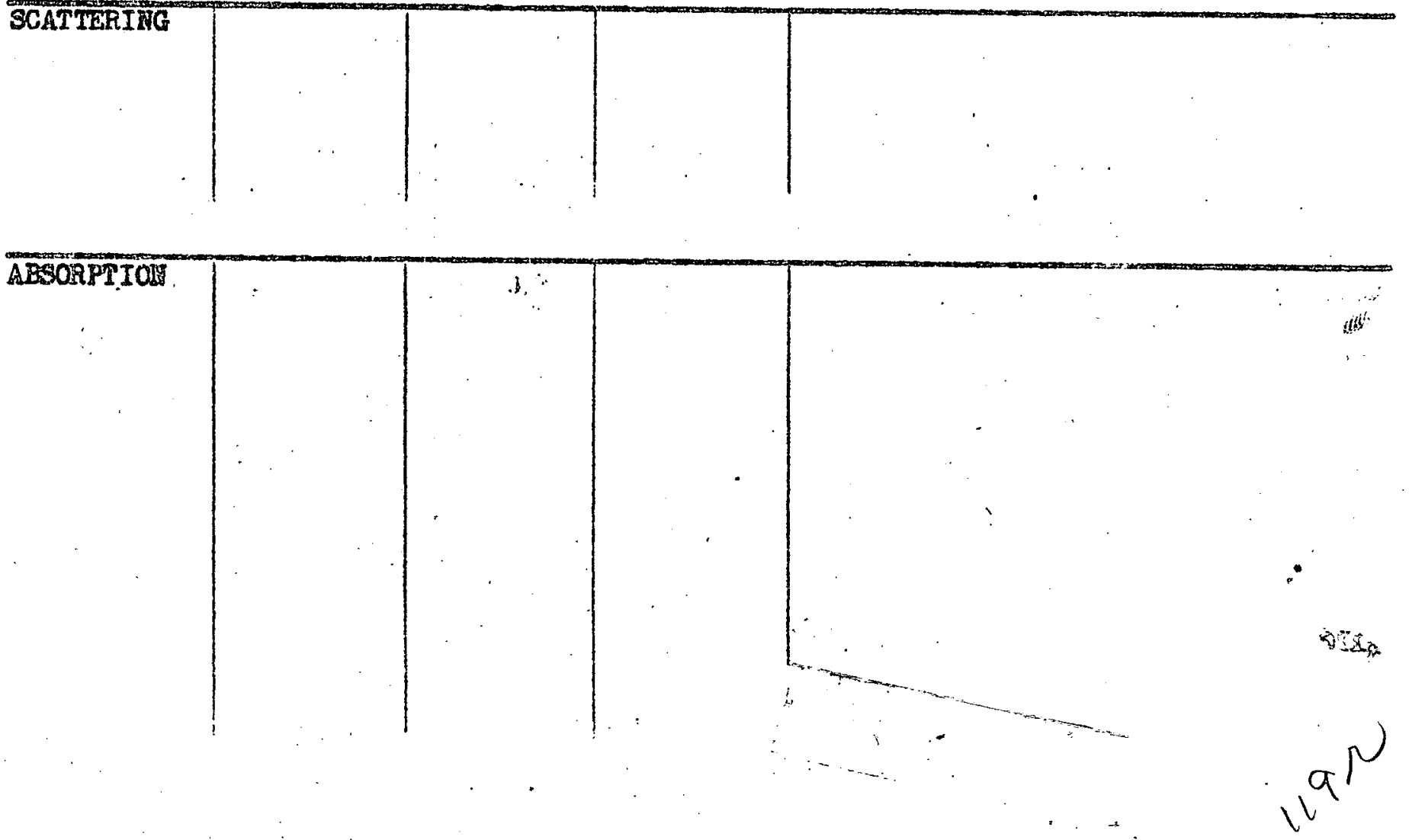


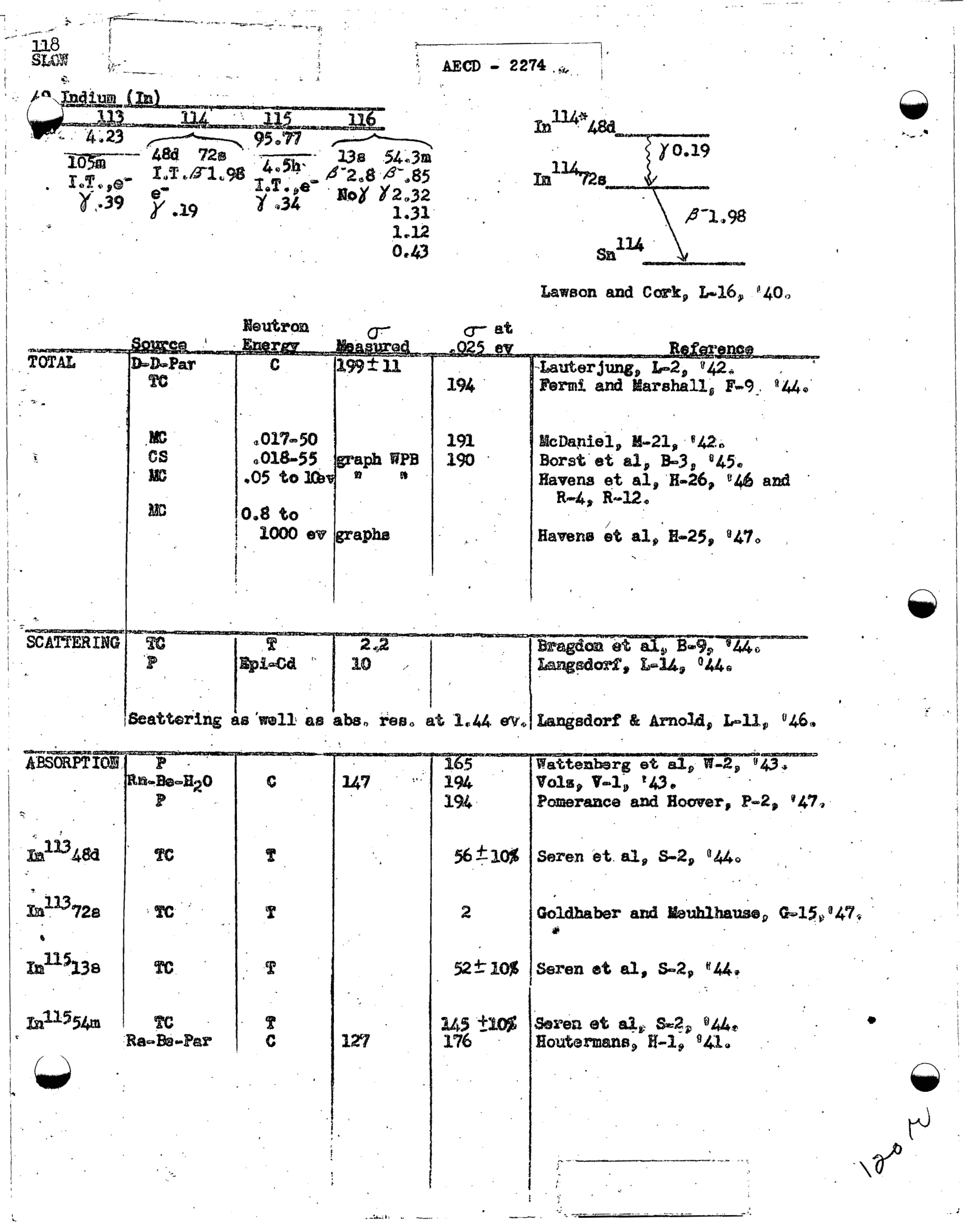


50 of $(5 n)$

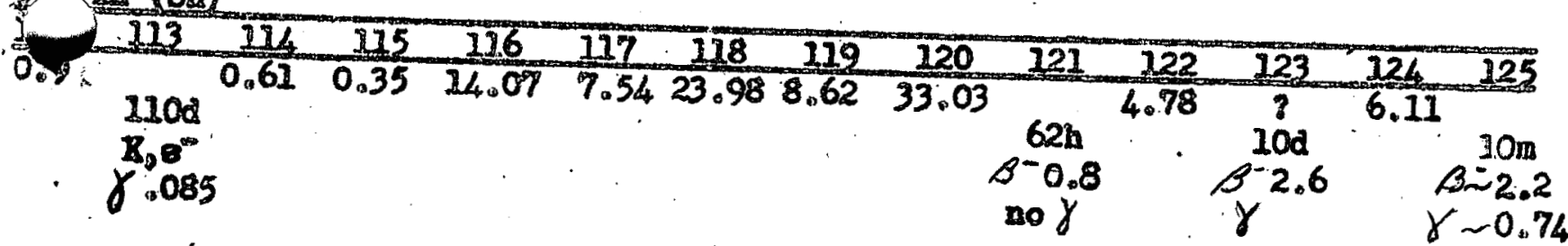

$\frac{\text { Ongssigned act101ties }}{40 \mathrm{~m}}$

$\beta^{-} \beta^{-} \mathscr{A}^{-} \beta^{-}$

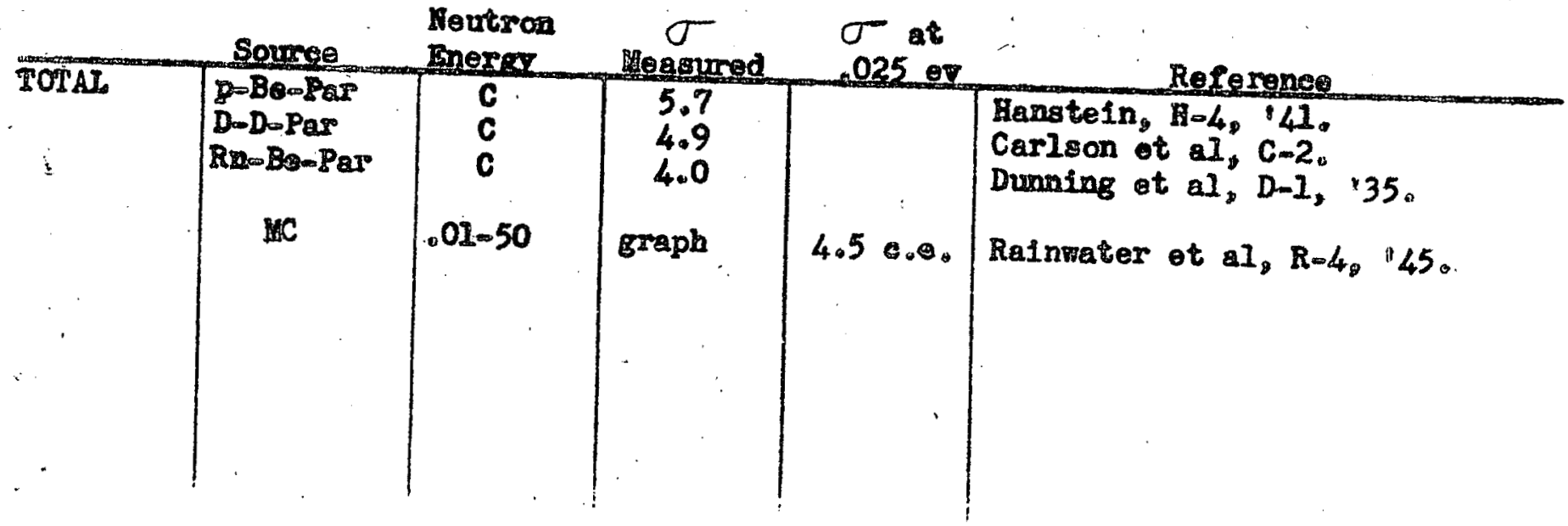

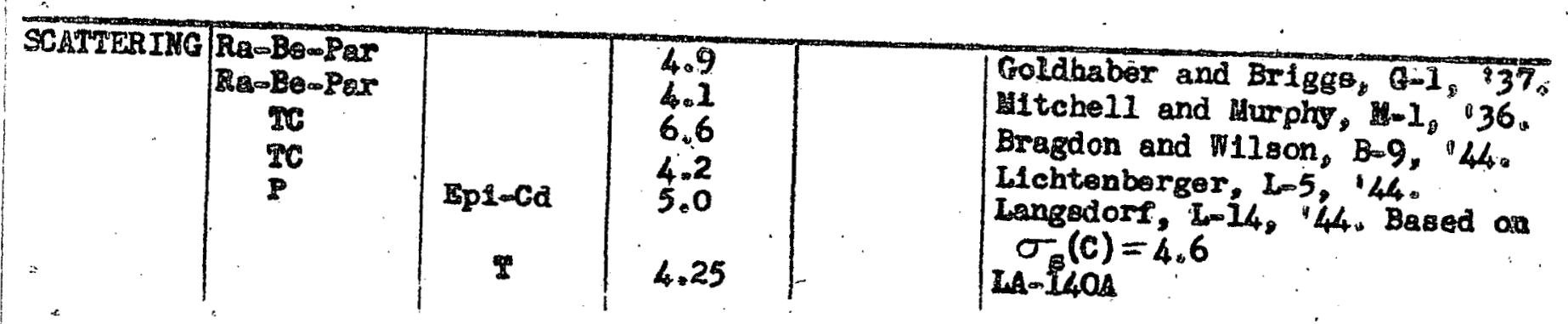

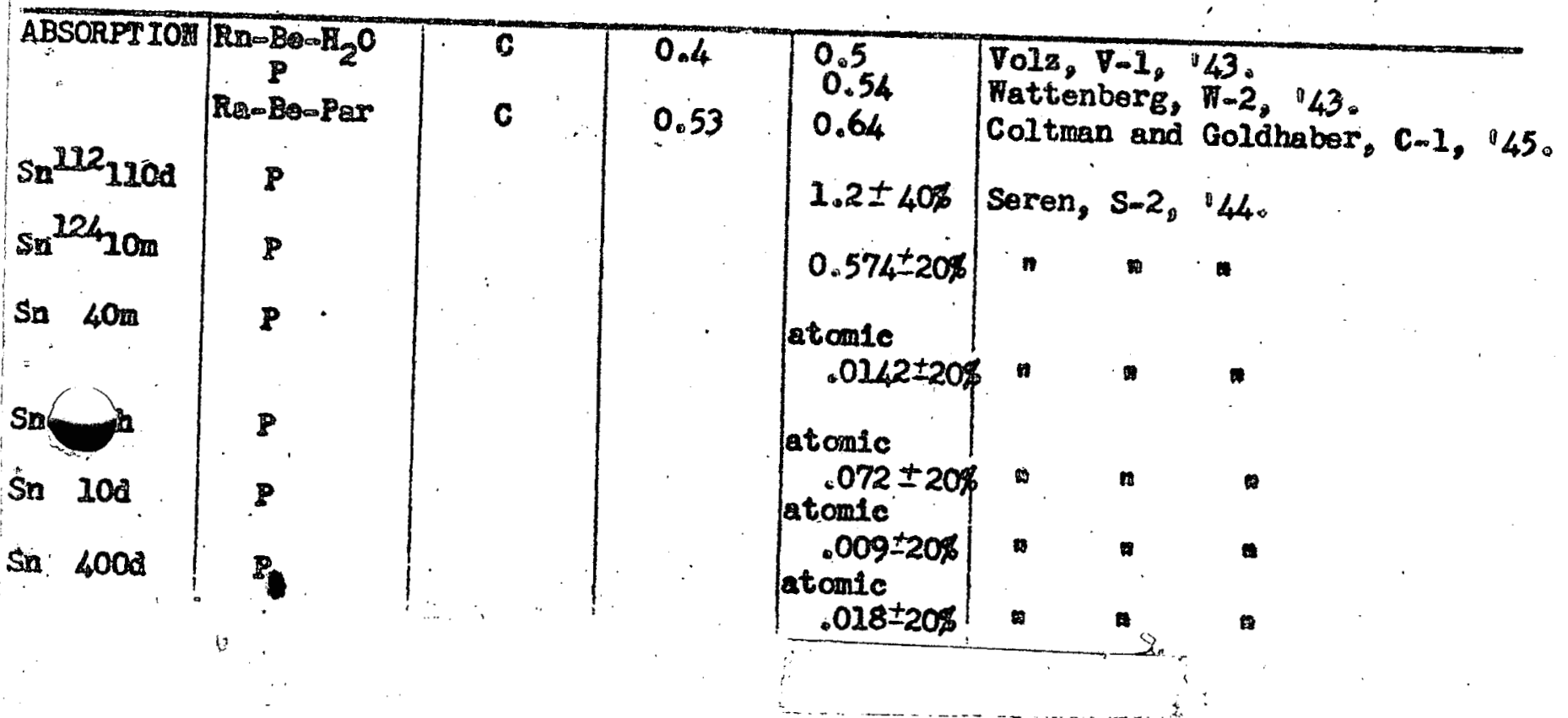


Resonanes

$$
\begin{array}{ll}
E_{0} & \frac{\sigma_{0} \Gamma}{5.8} \\
25 & \sim 12 \\
21 & \sim 35 \\
& \sim 35
\end{array}
$$

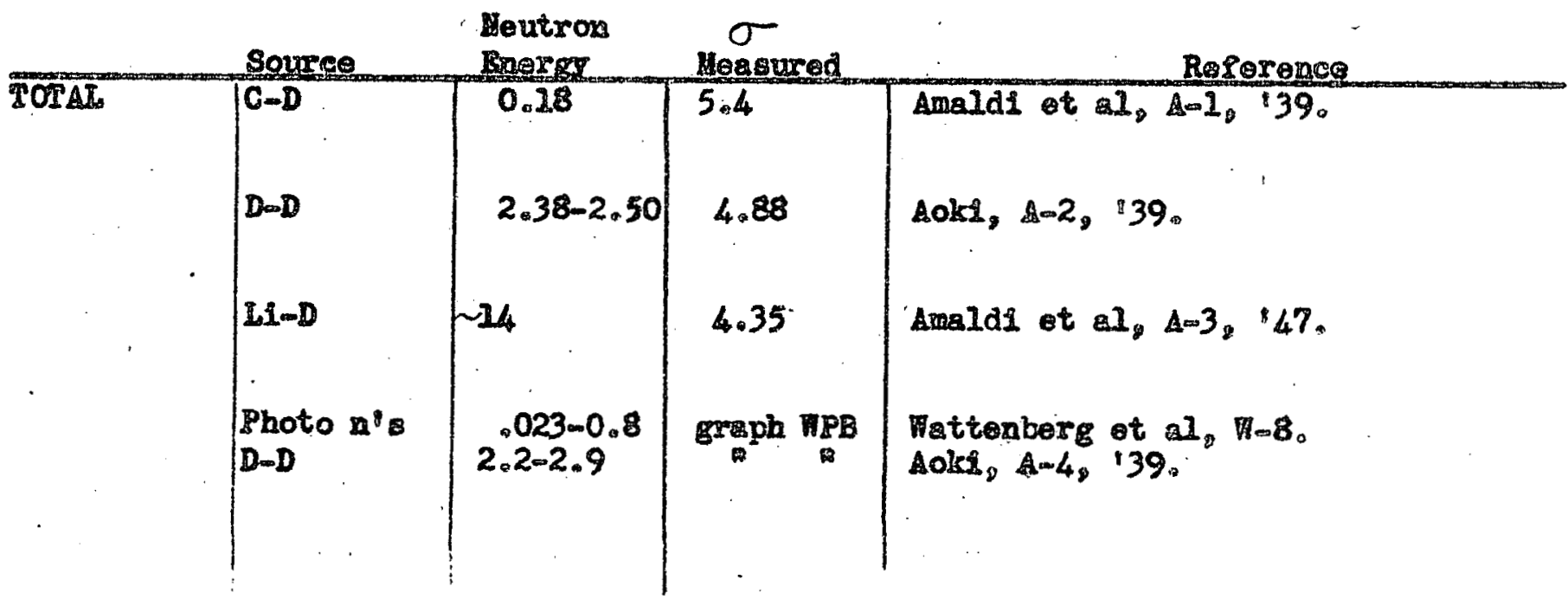

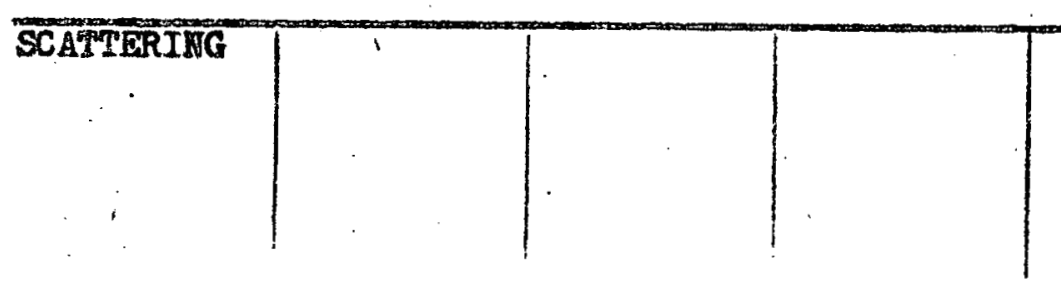

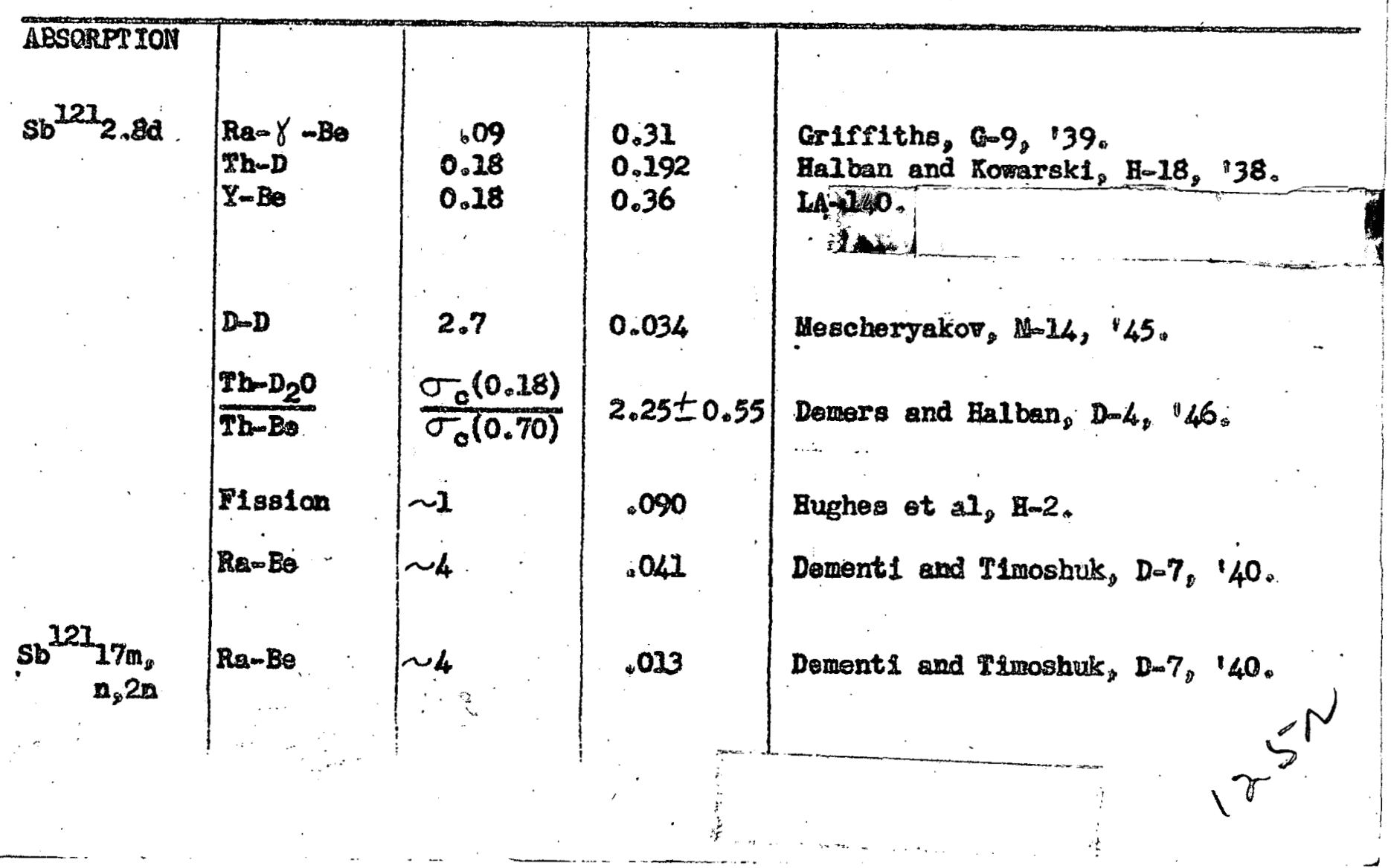




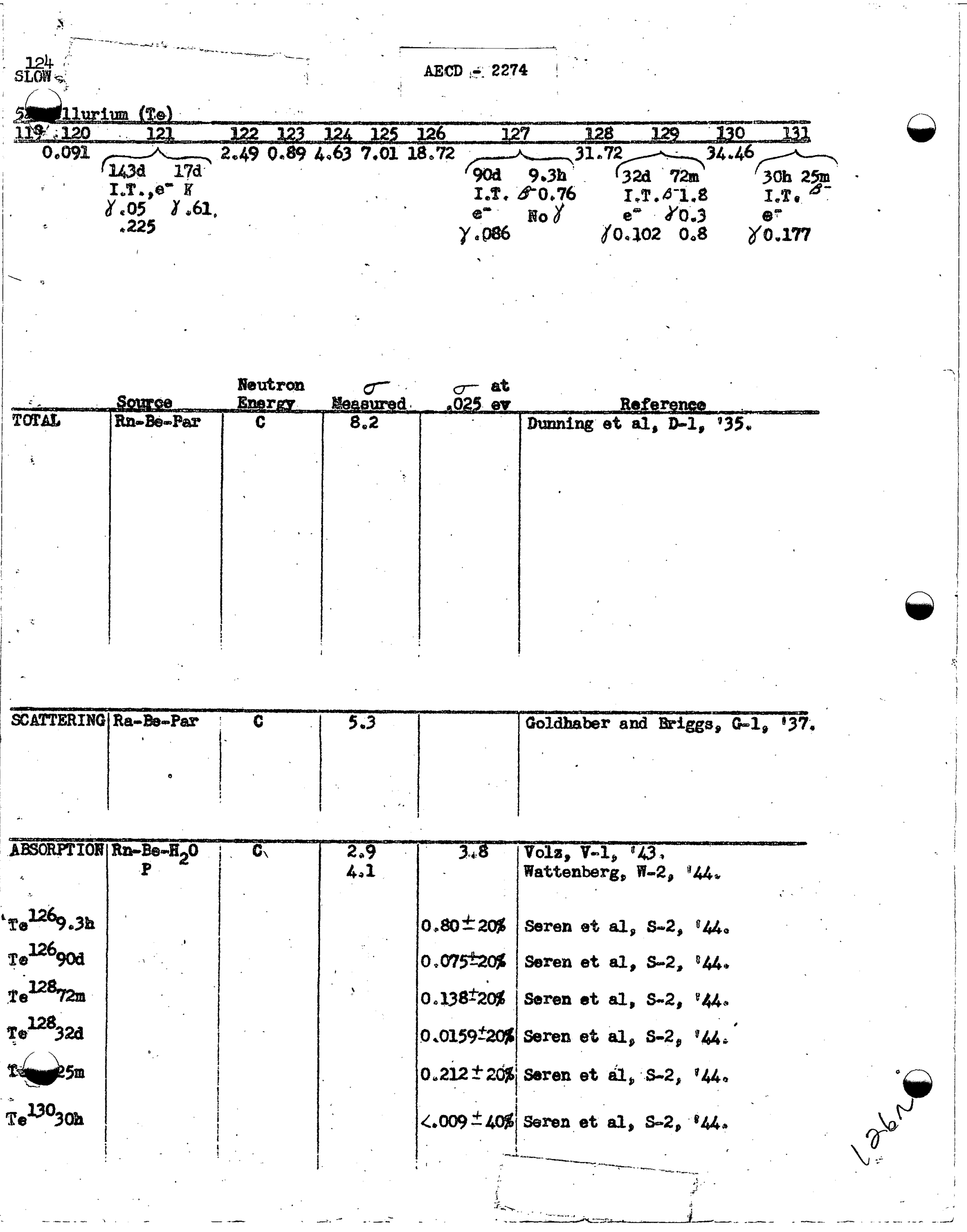




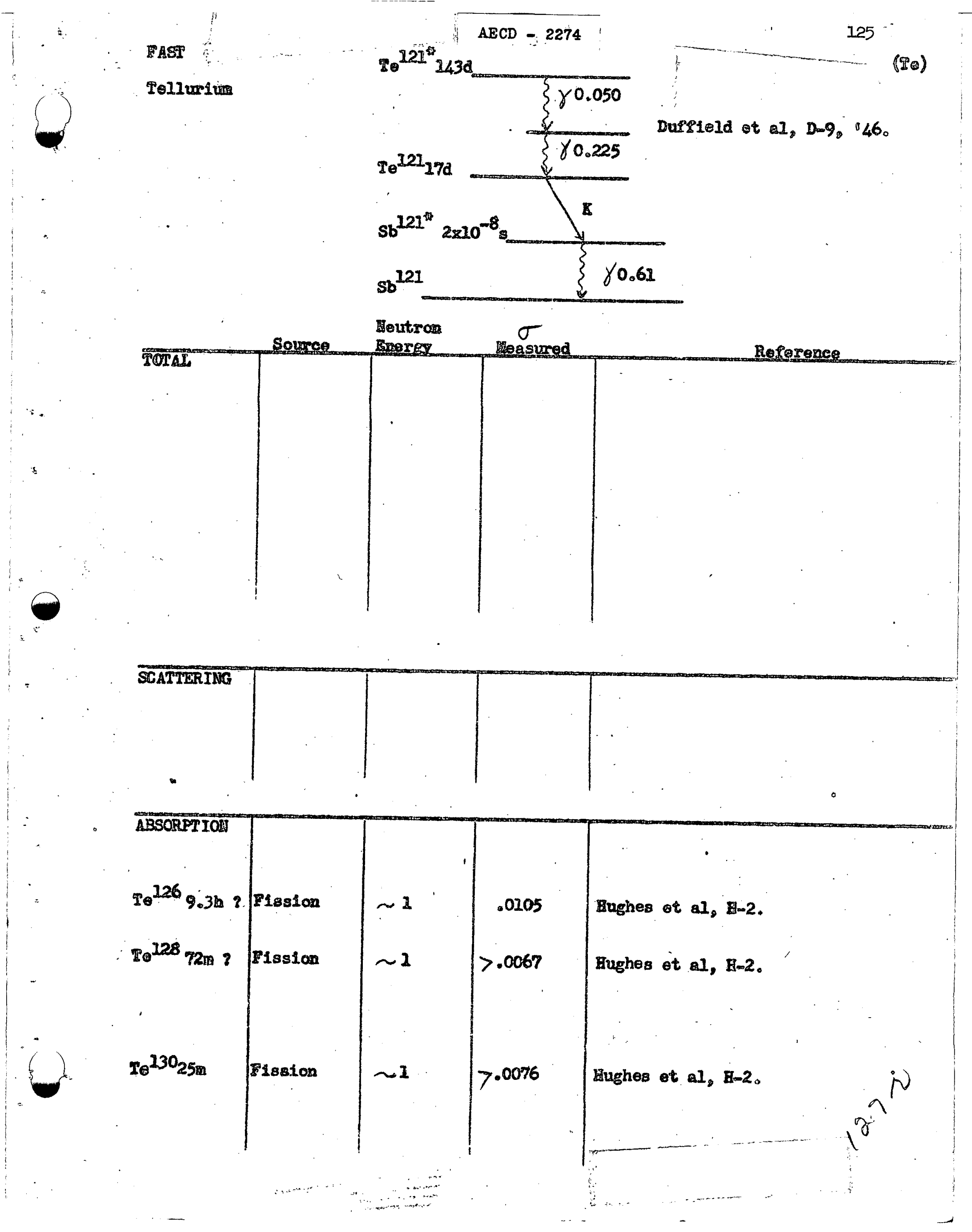




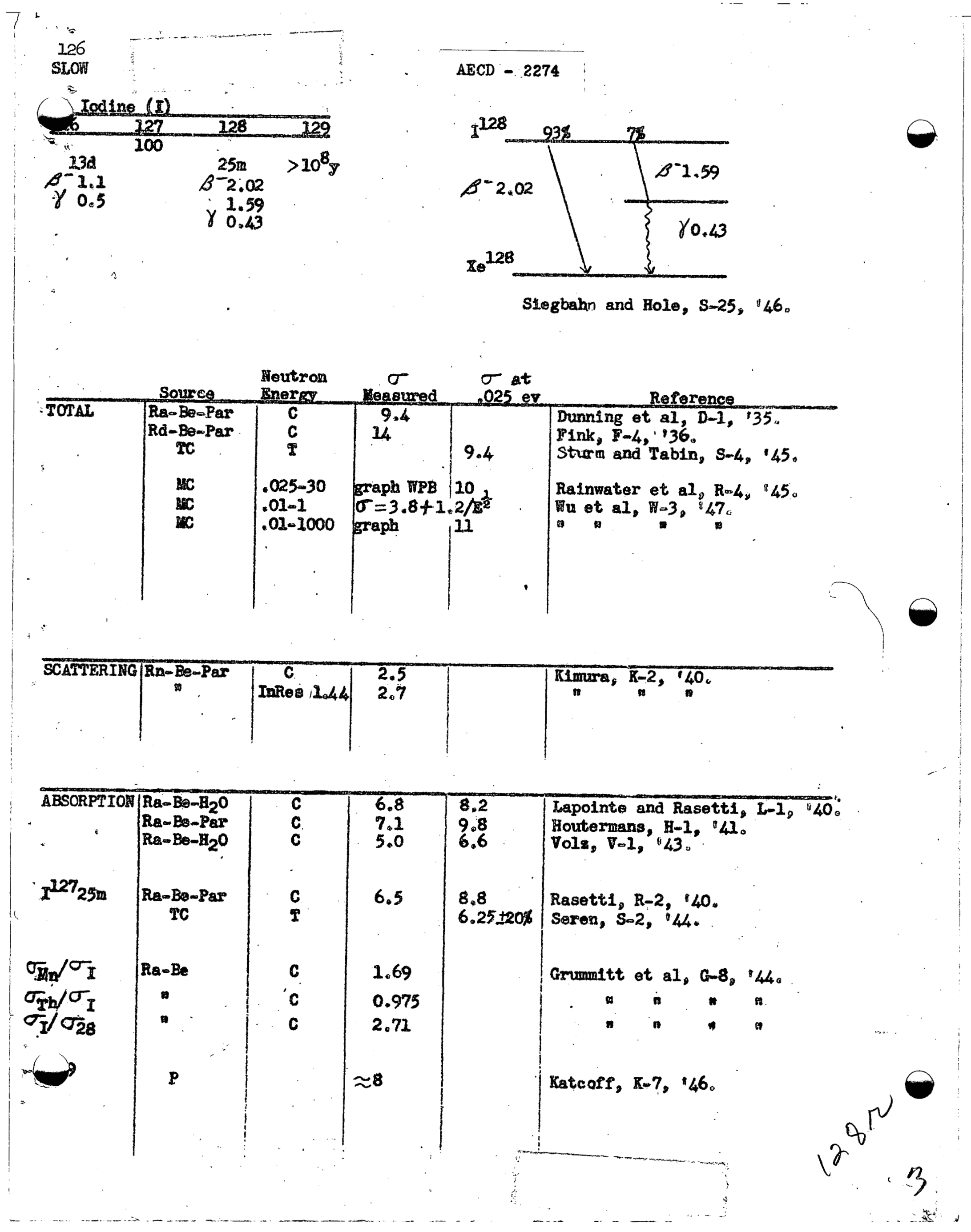


Resonances

$$
\begin{array}{cc}
\underline{s}_{20.6 \pm 0.4} & \frac{\sigma_{0} \Gamma^{2}}{\sim_{4}^{4}} \\
42 \pm 2 & \sim 135 \\
42 \pm 2 & \sim 135
\end{array}
$$

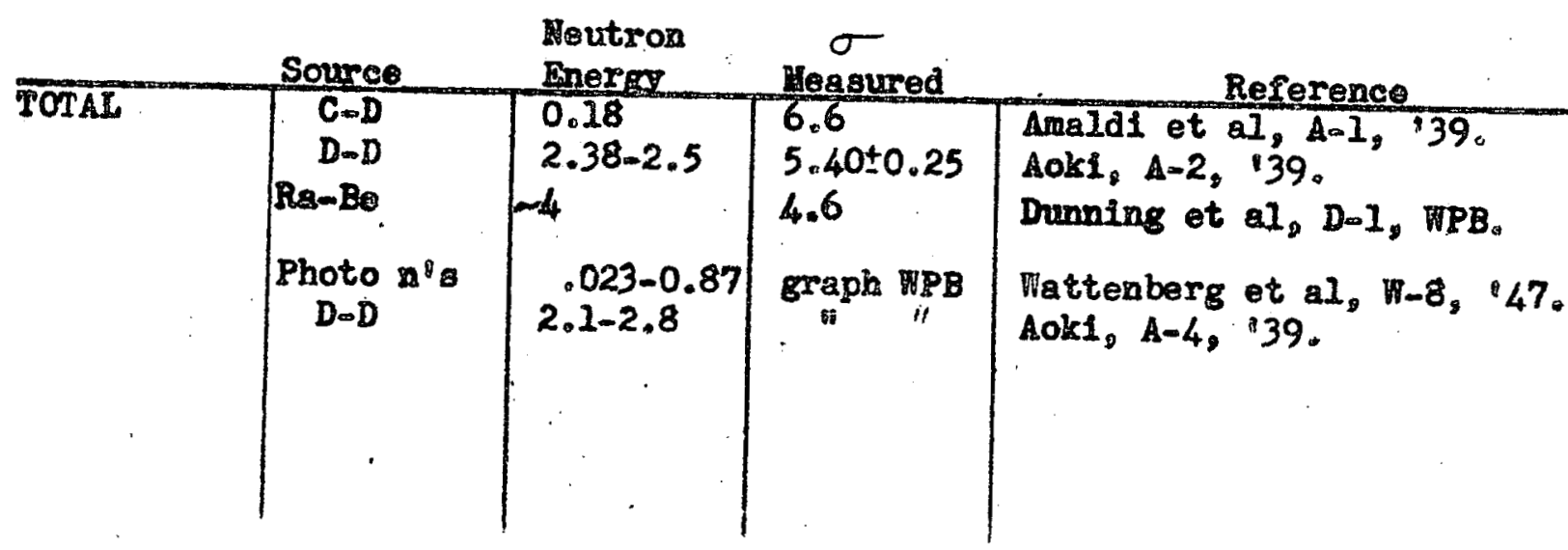

SCATTERING

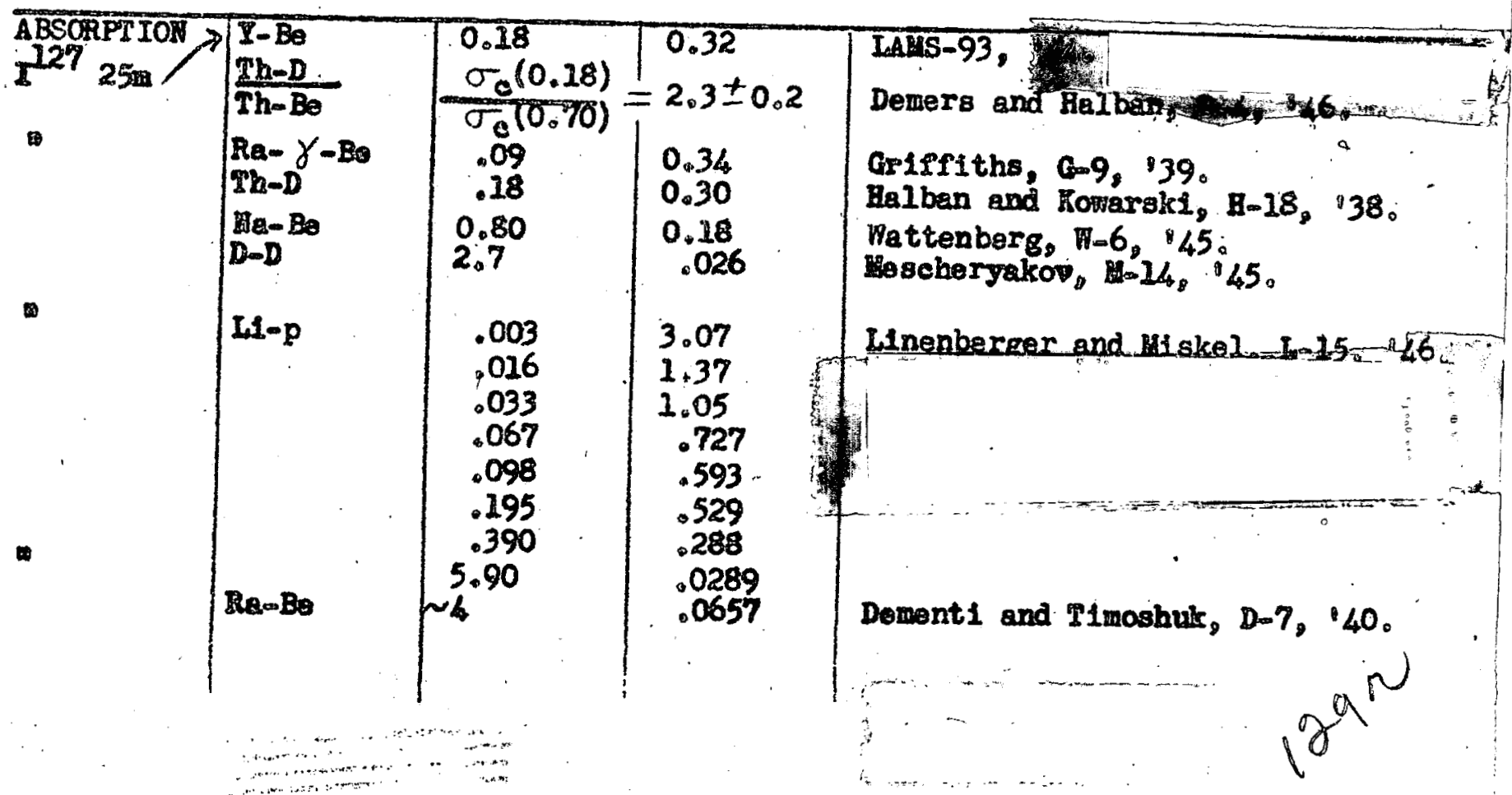



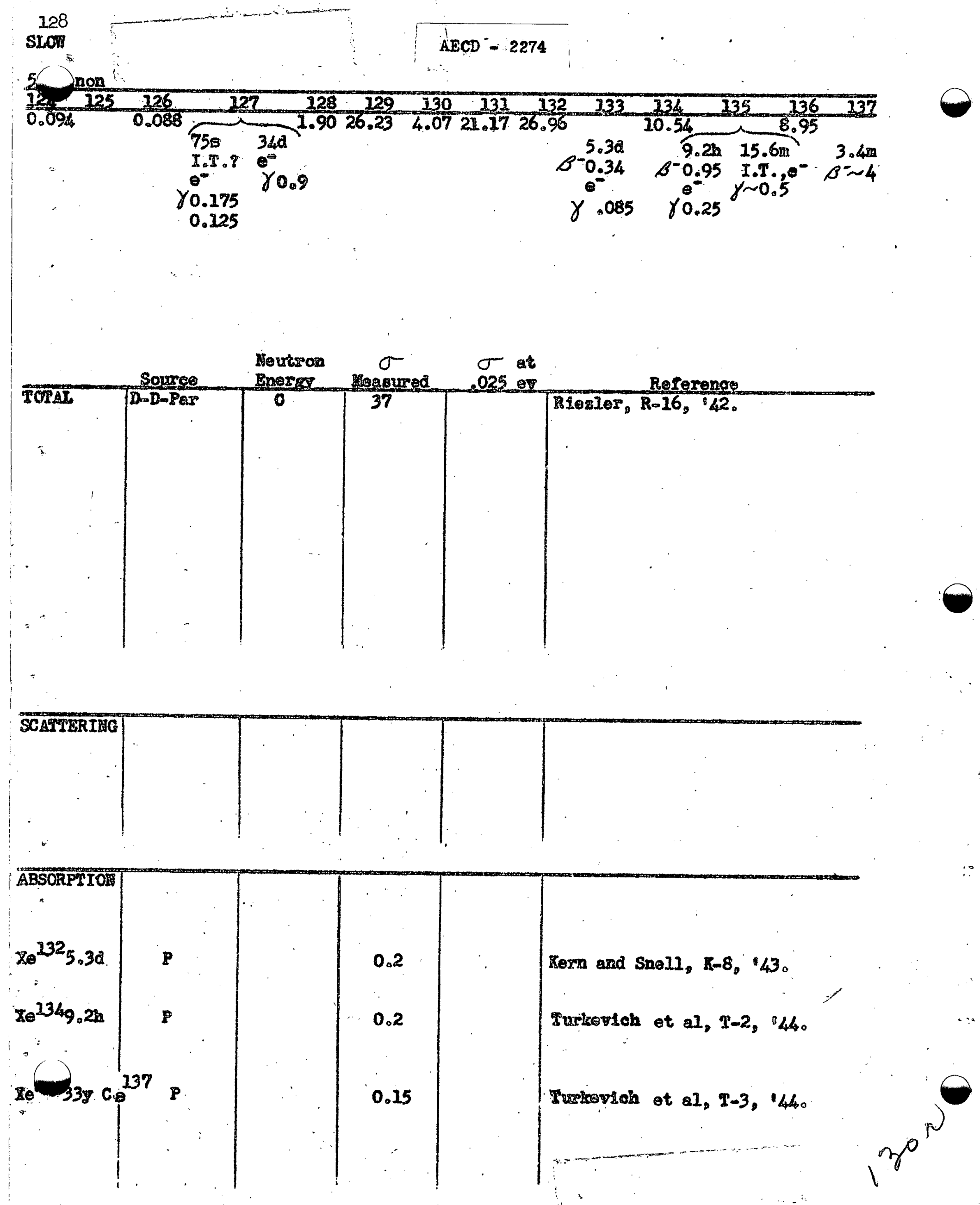

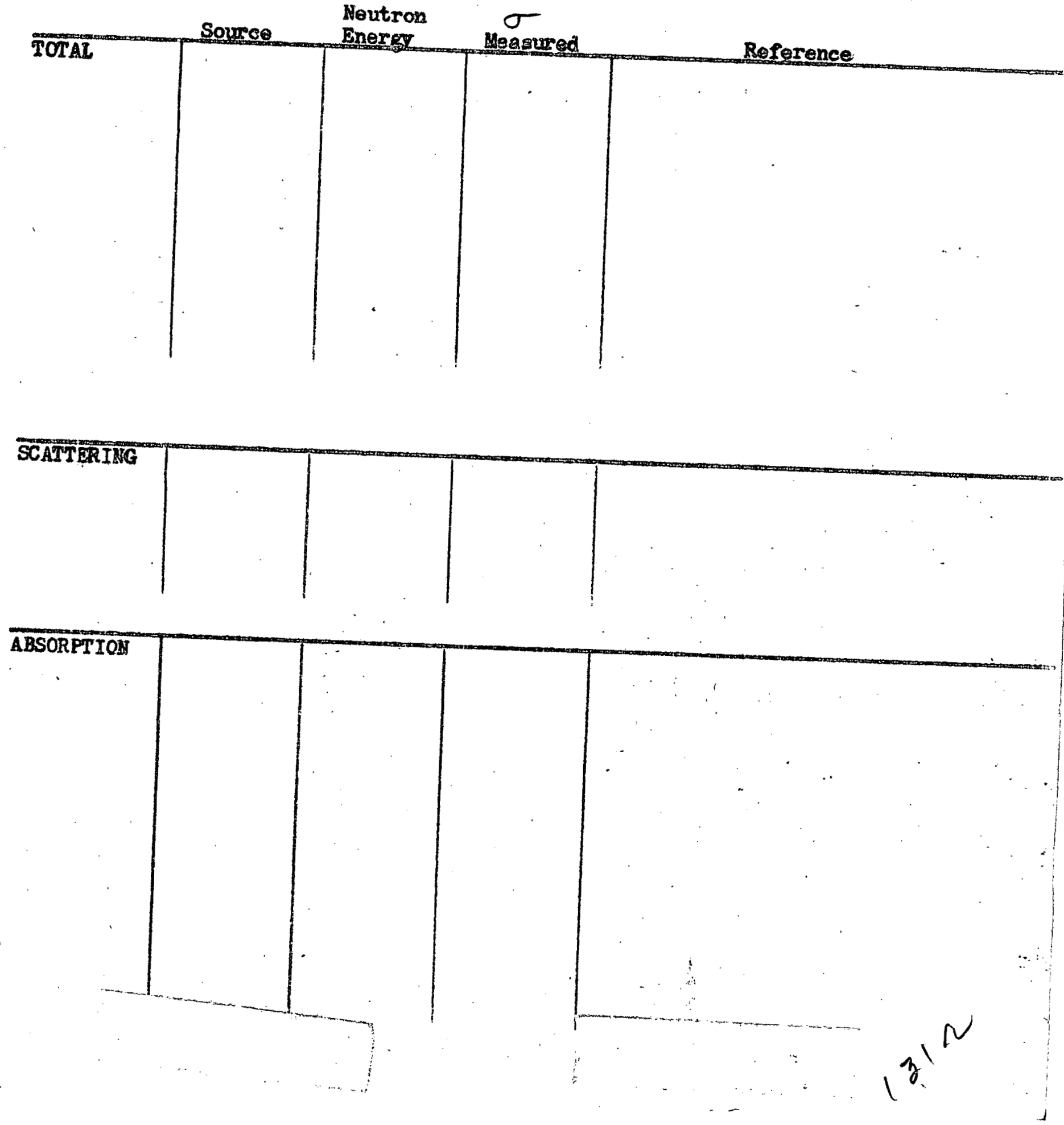


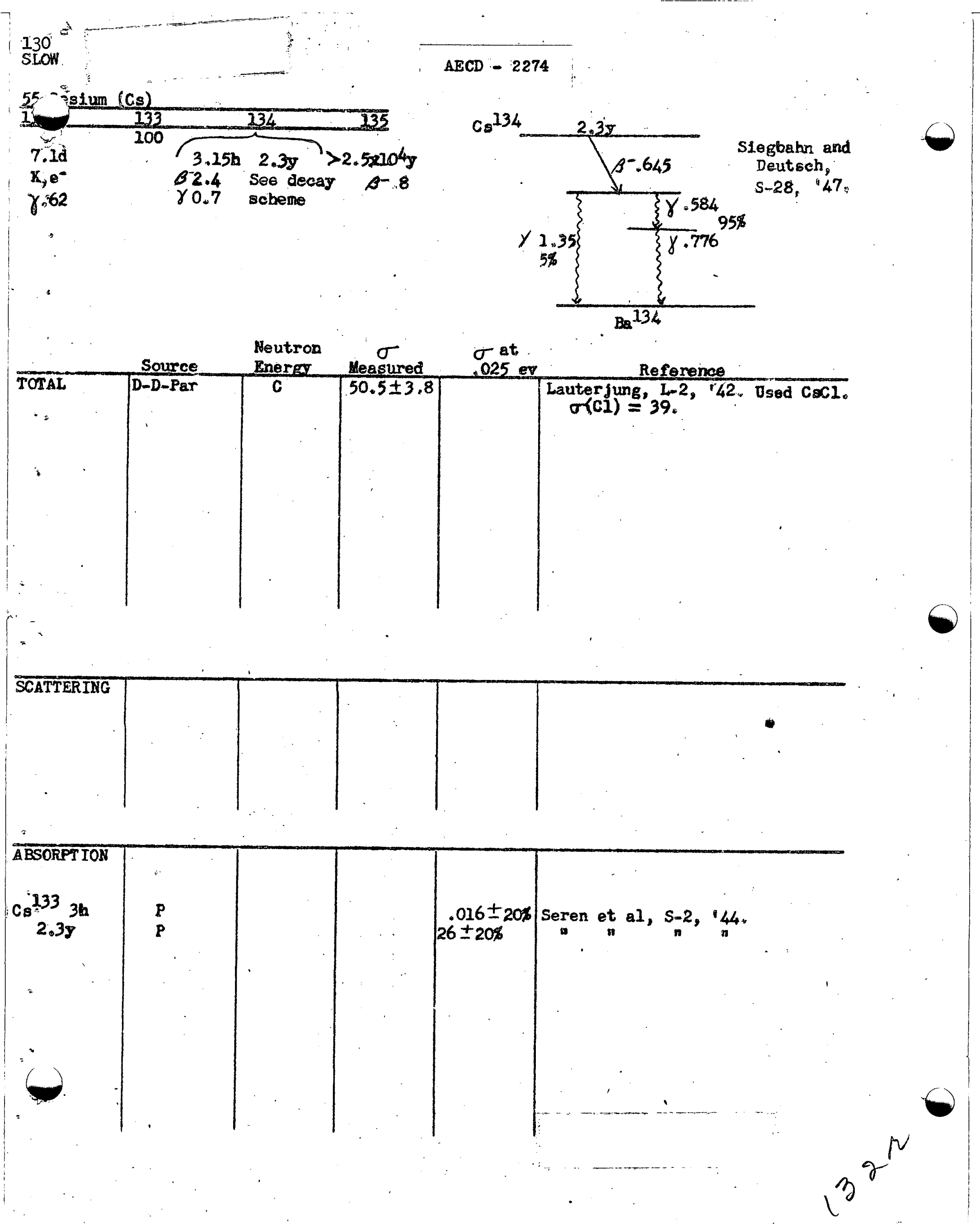




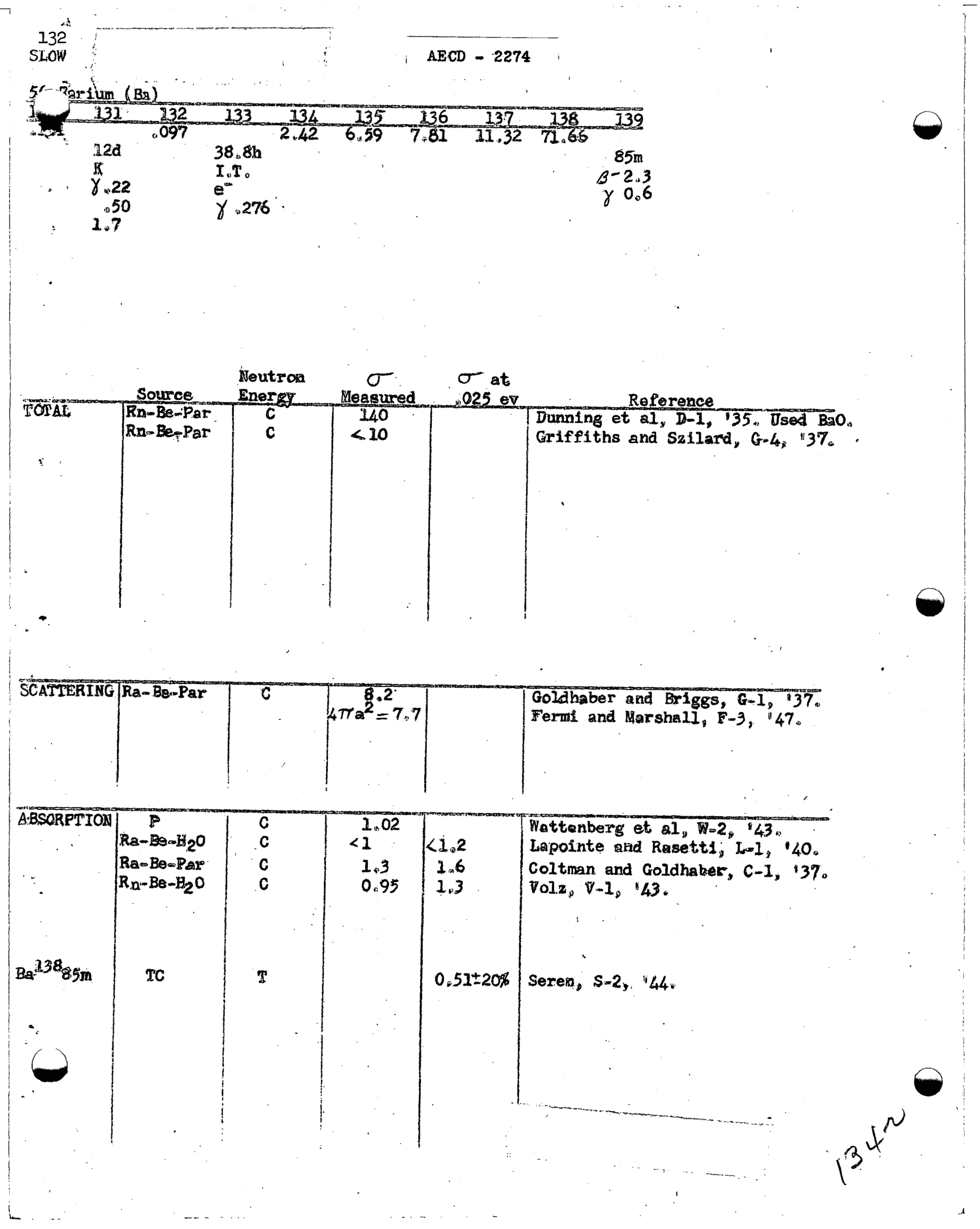




\begin{tabular}{|c|c|c|c|c|}
\hline & Source & $\begin{array}{l}\text { Heutron } \\
\text { Energy }\end{array}$ & $\begin{array}{c}\sigma \\
\text { Hegaured }\end{array}$ & Reference \\
\hline TOTAX & $D \rightarrow D$ & 2.4 & 6.19 & Aok1, $A-2,139$ \\
\hline
\end{tabular}
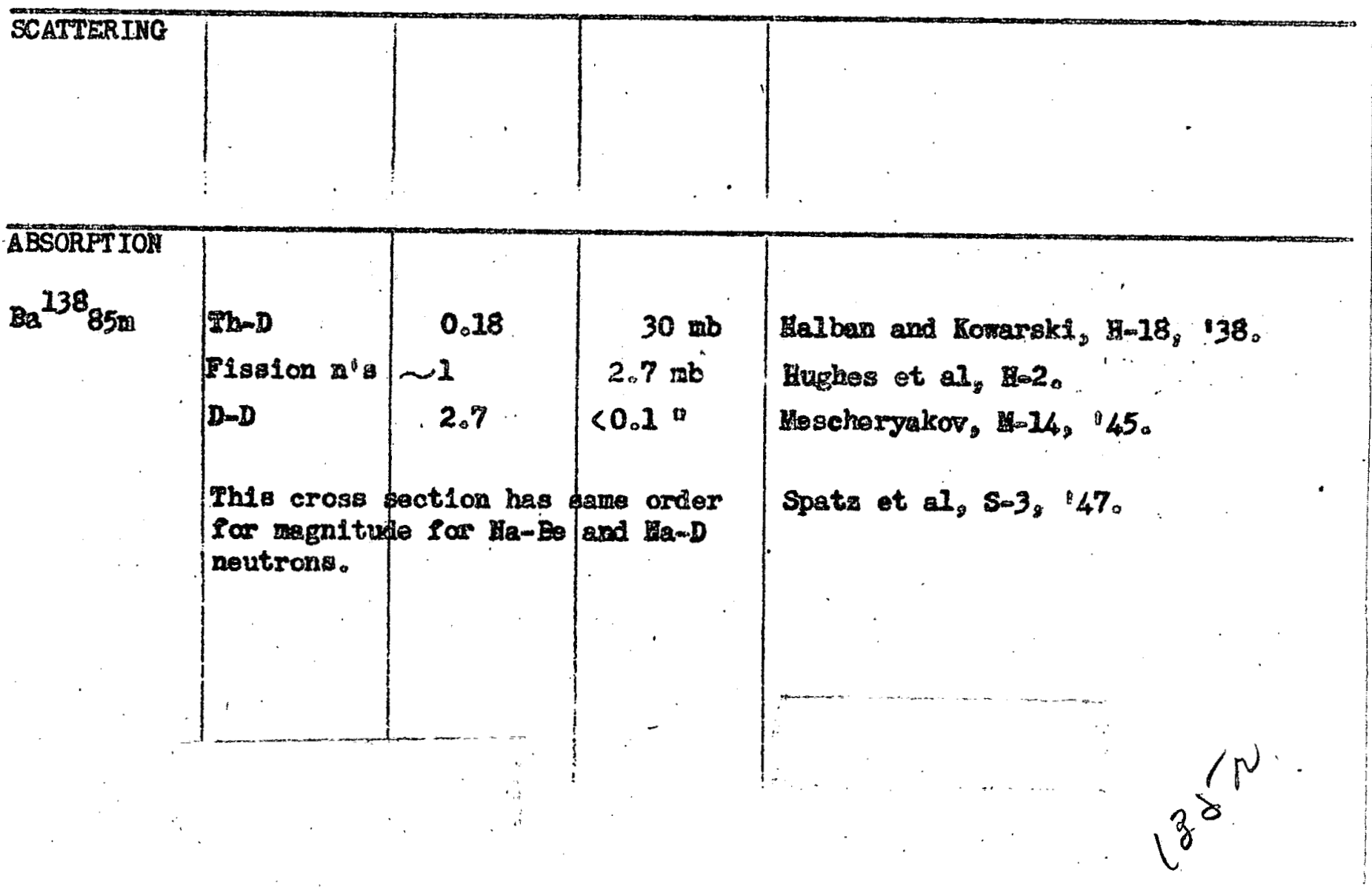


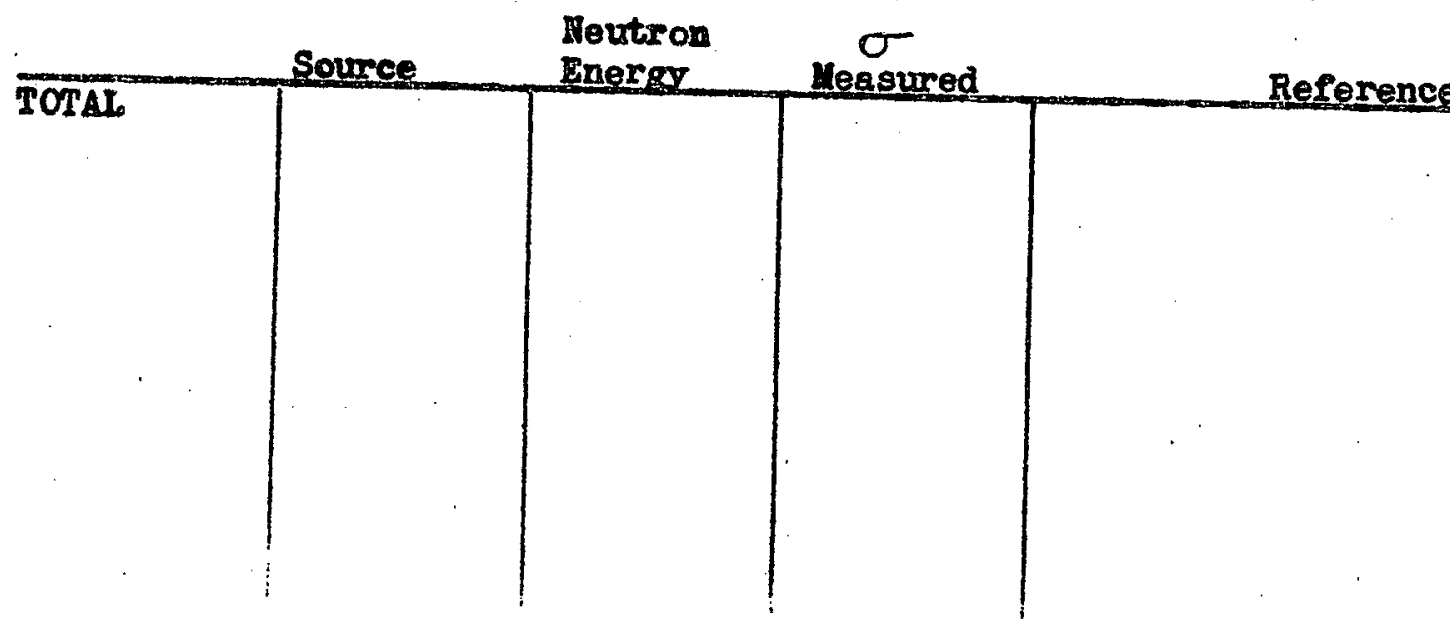

SCATTER ING
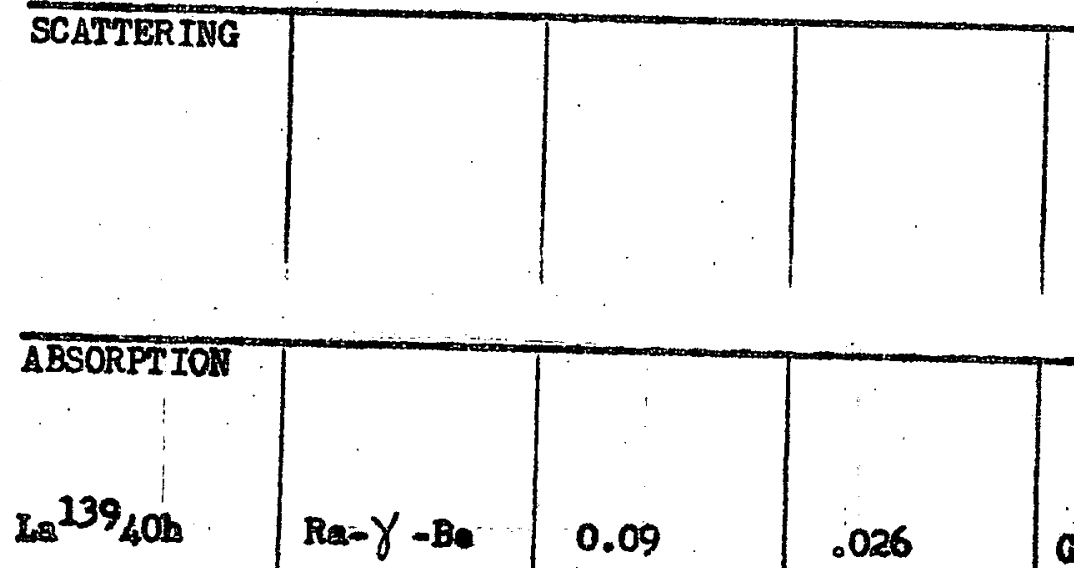

$(1)$
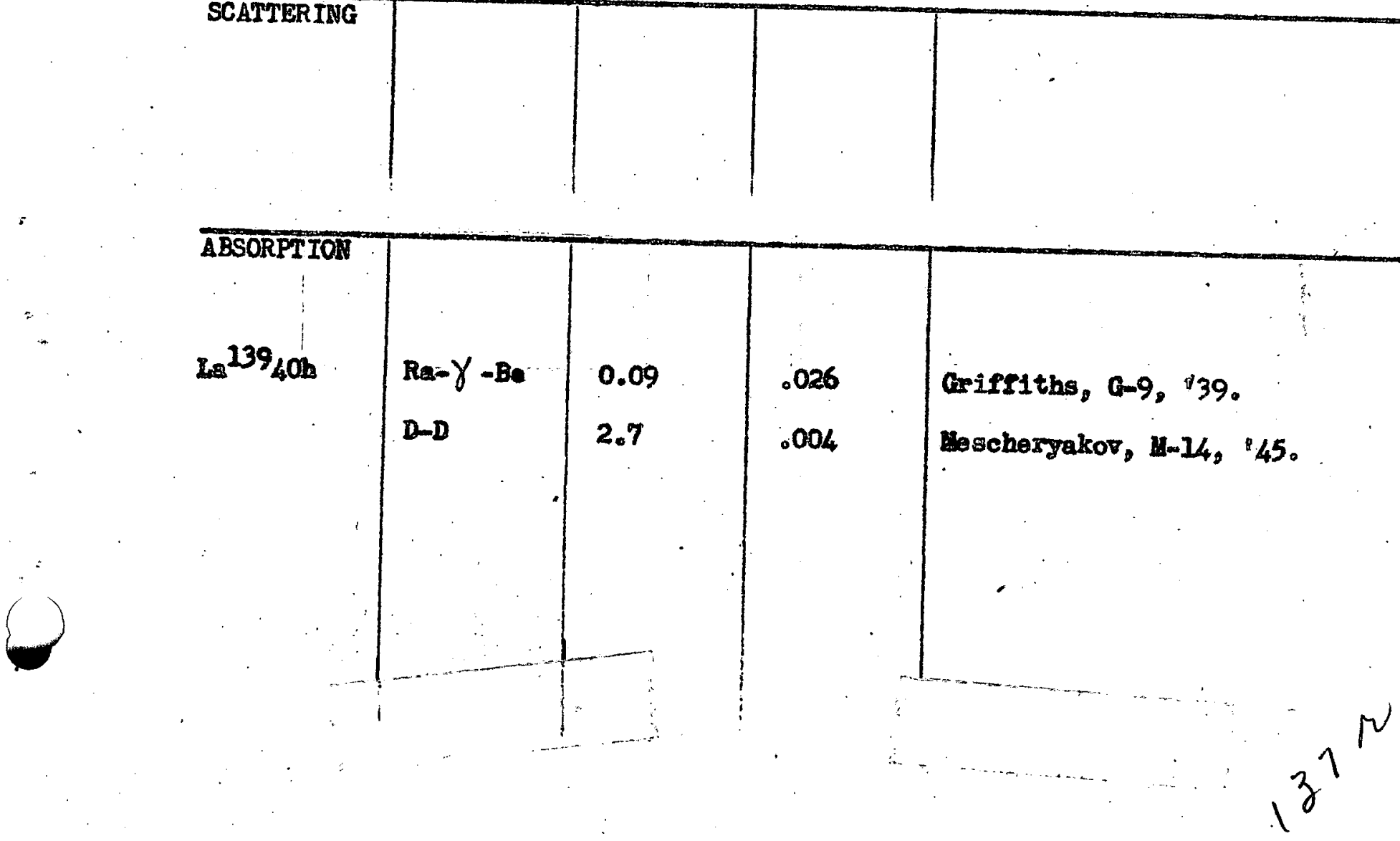


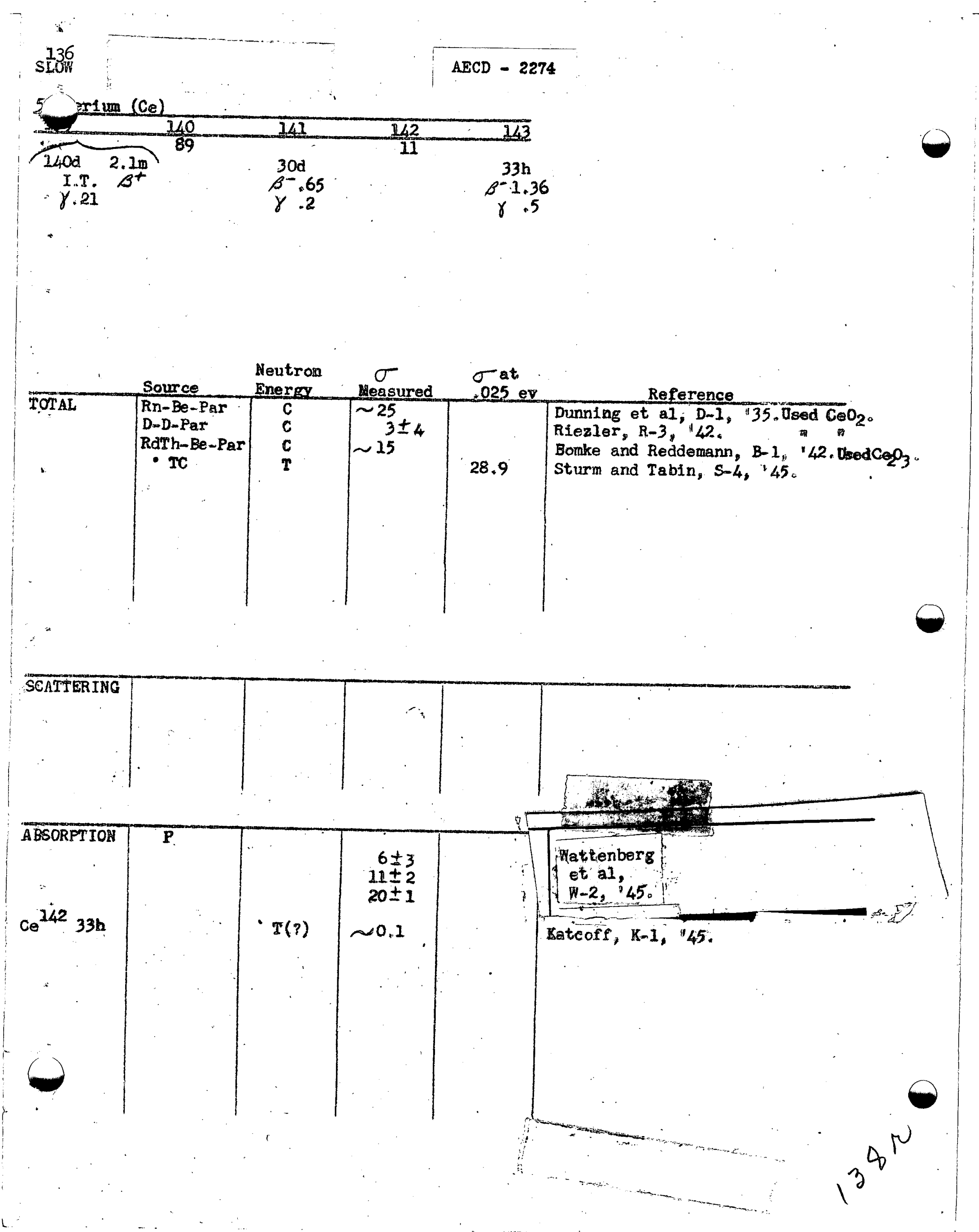




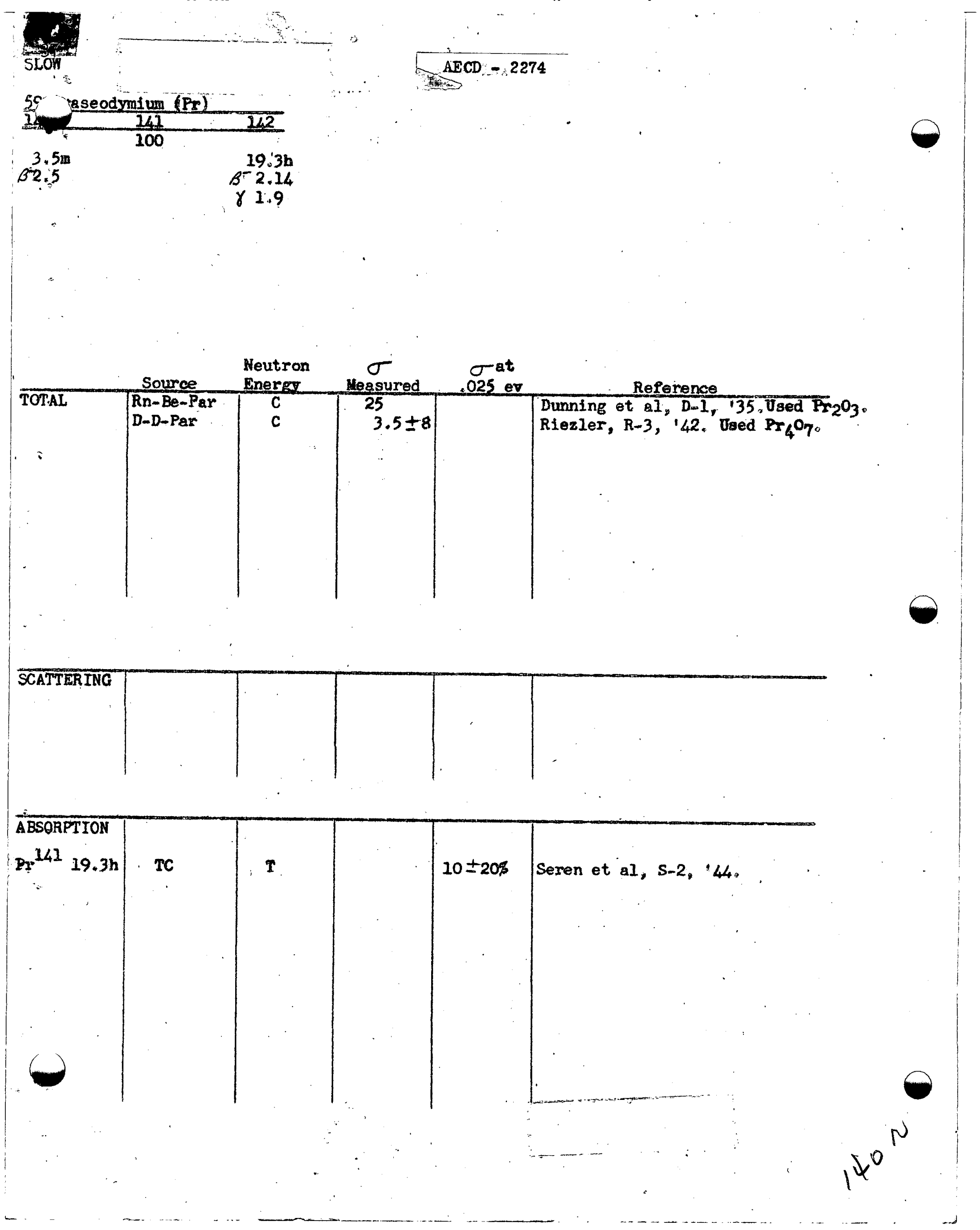




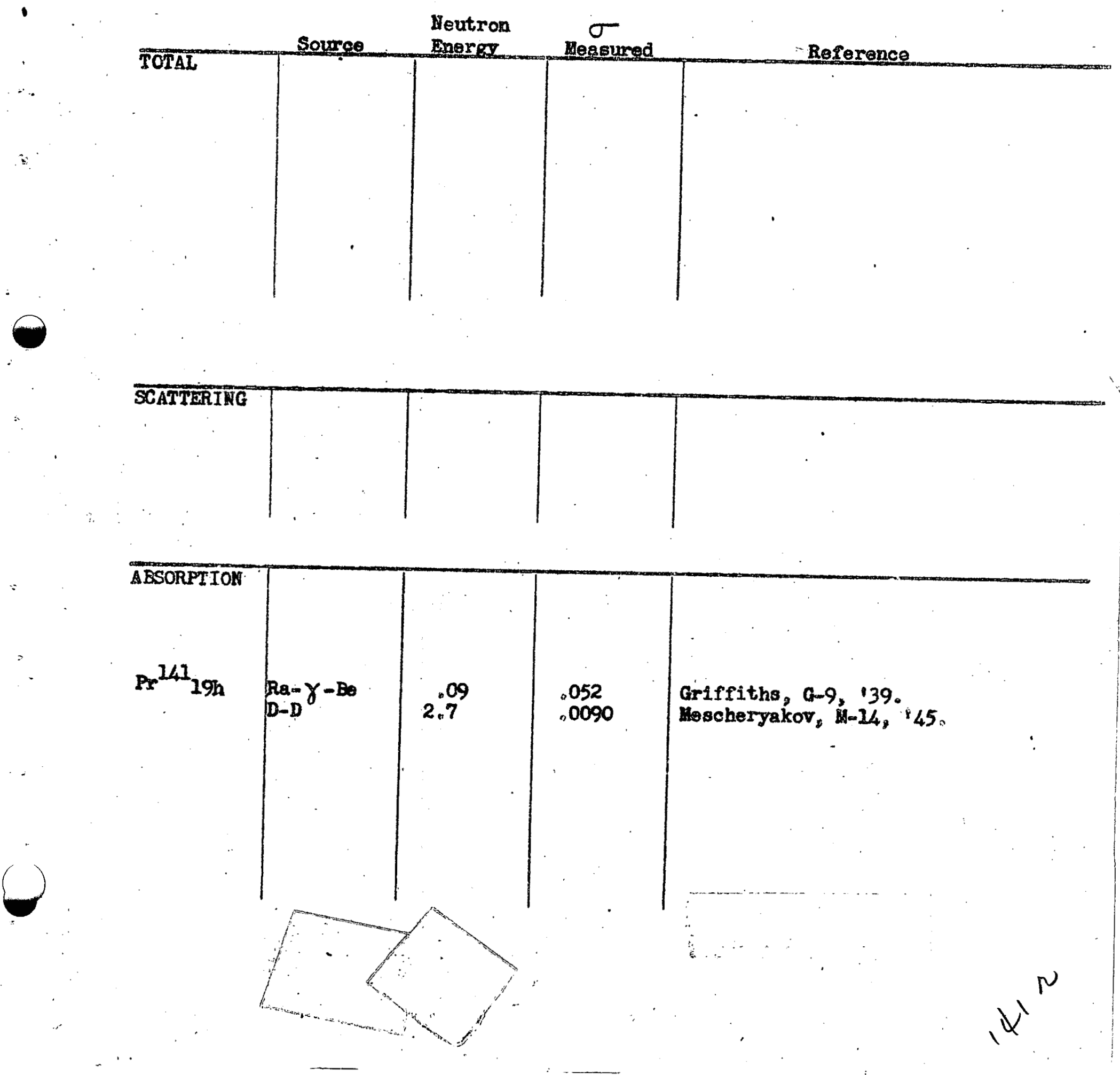




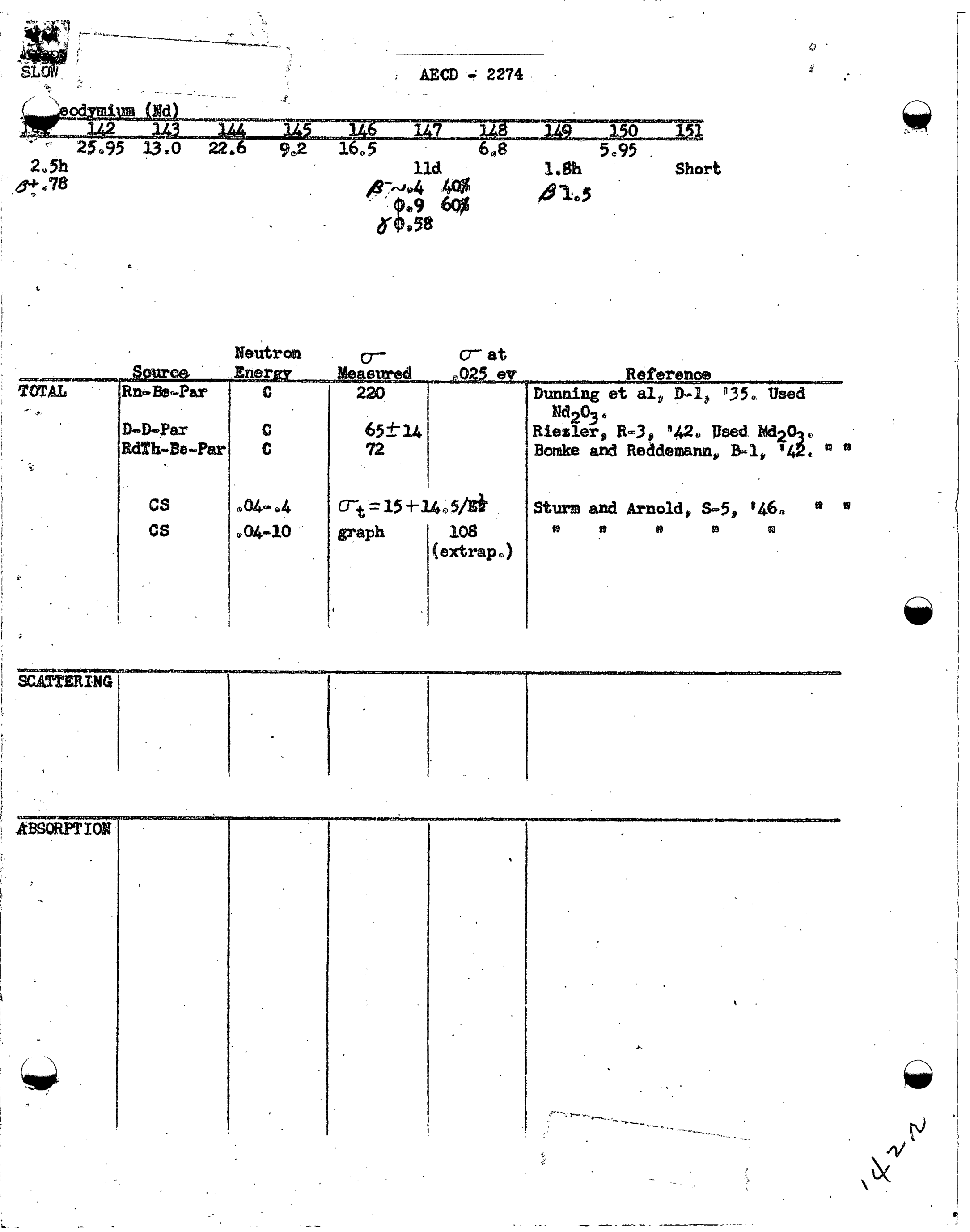




\section{I11inium (II)

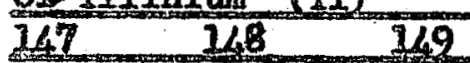 \\ $\sim B^{40} B^{5 a-5} B^{55 b}$

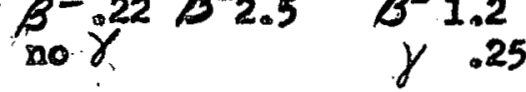
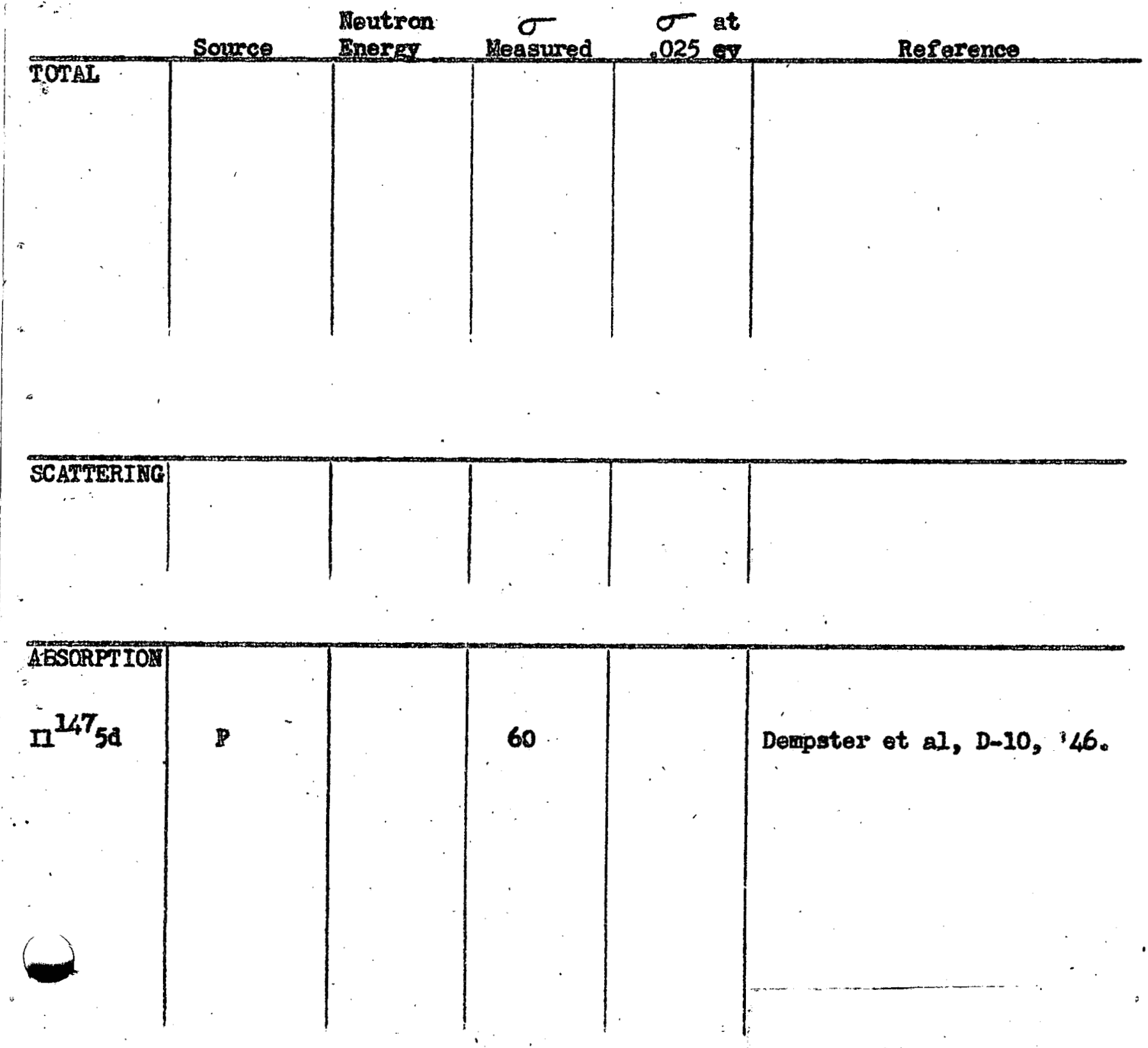

$x^{N}$ 


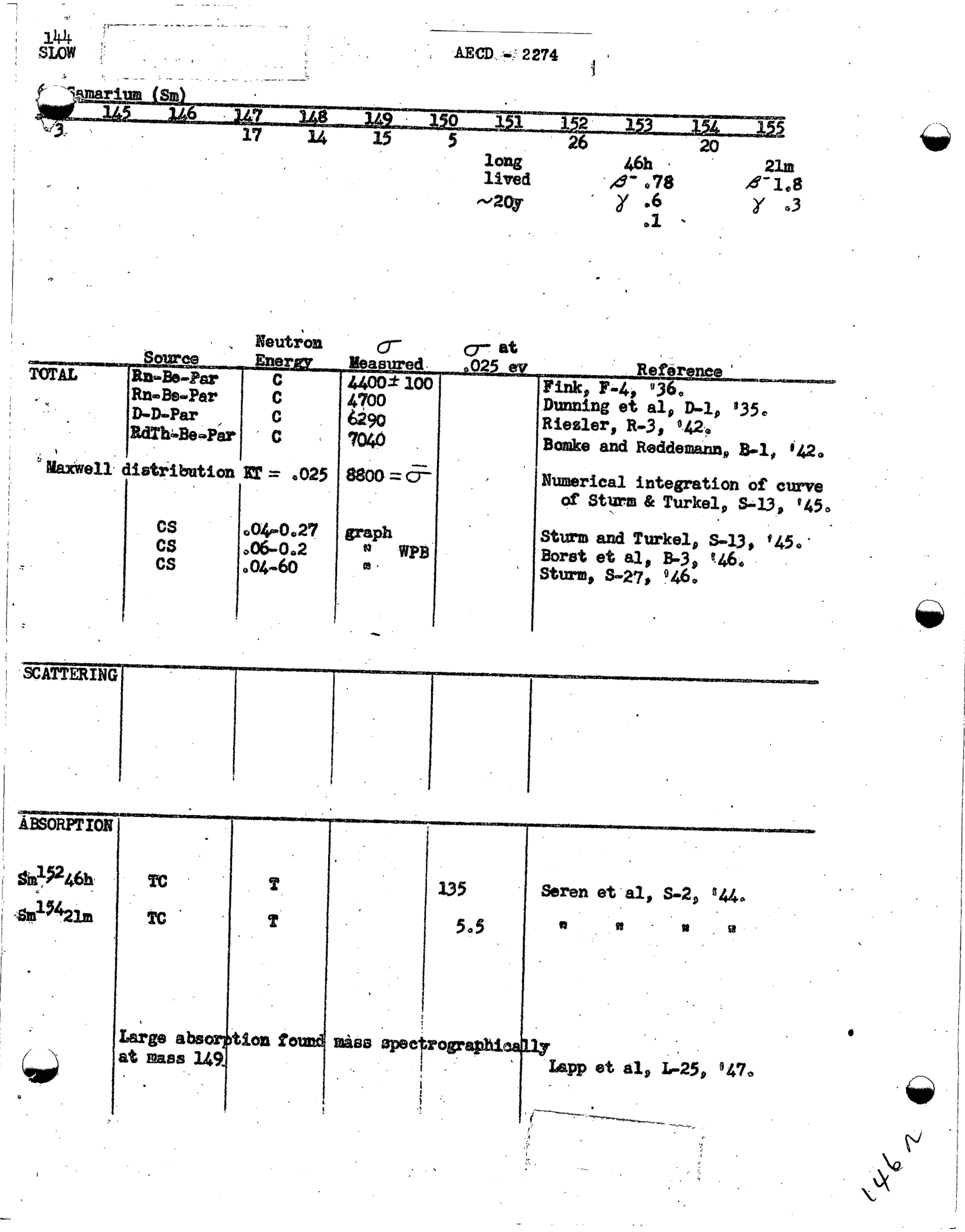




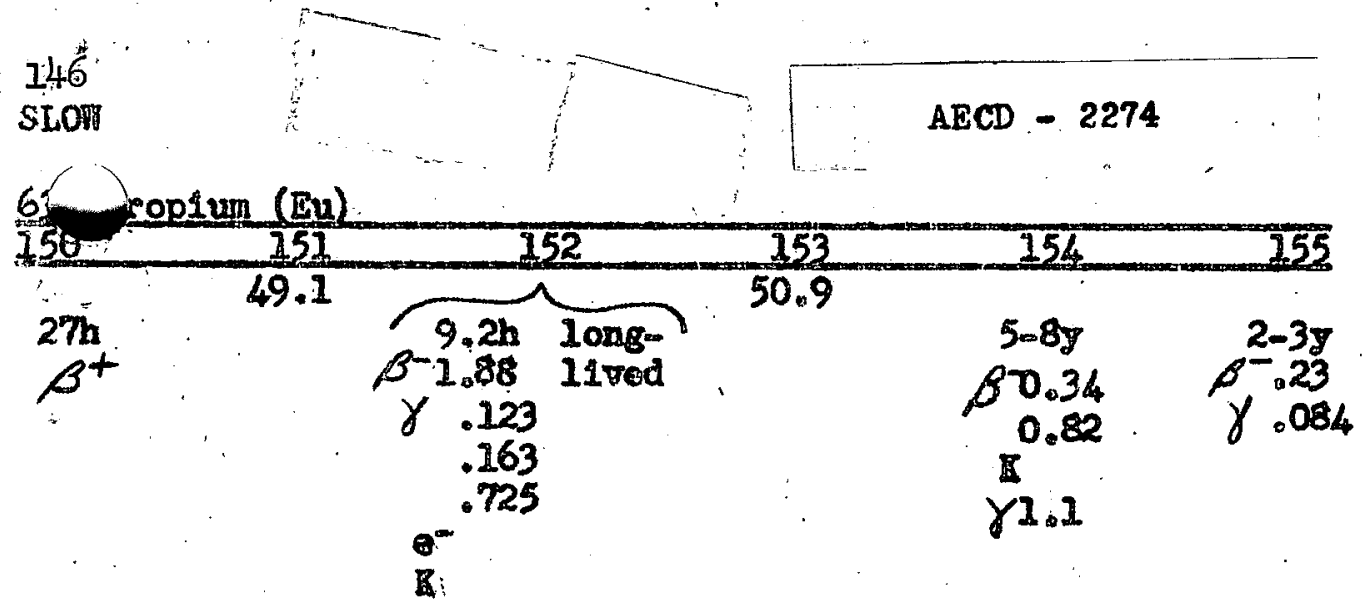

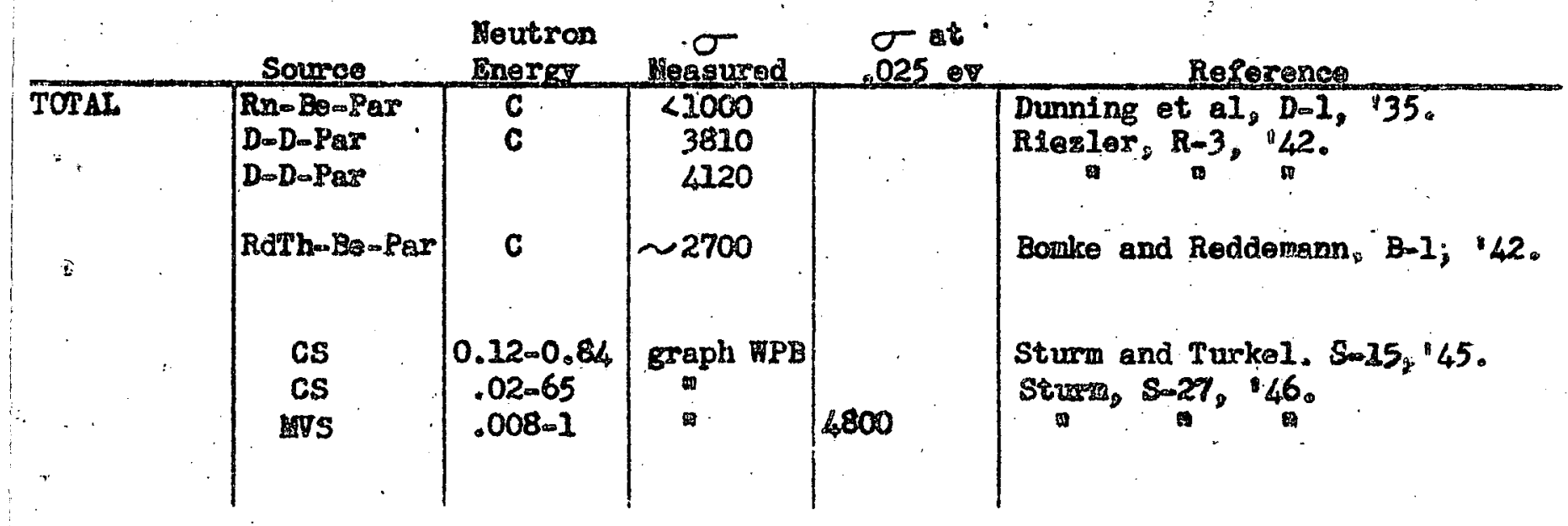

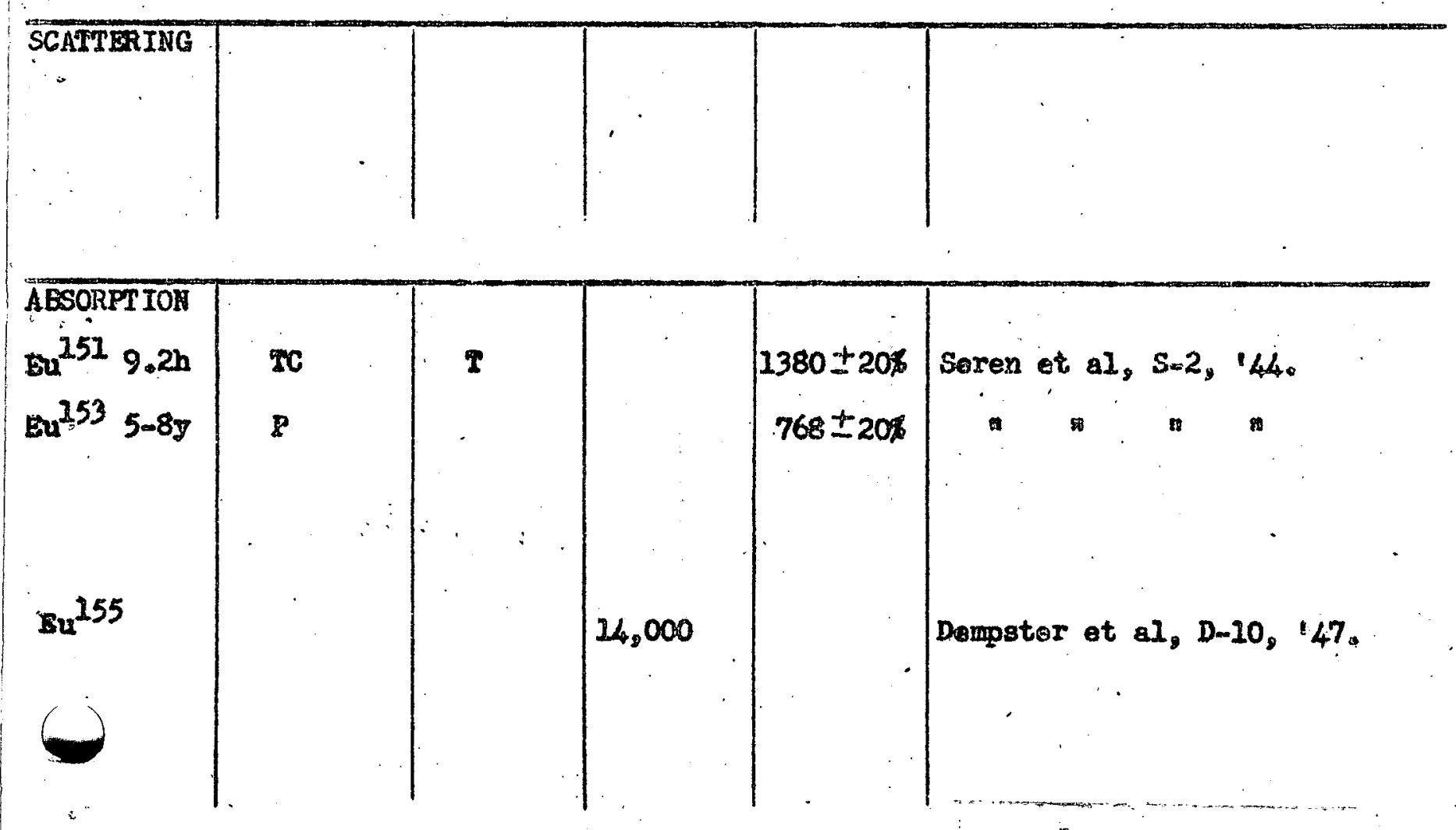

$i^{\infty}$ 


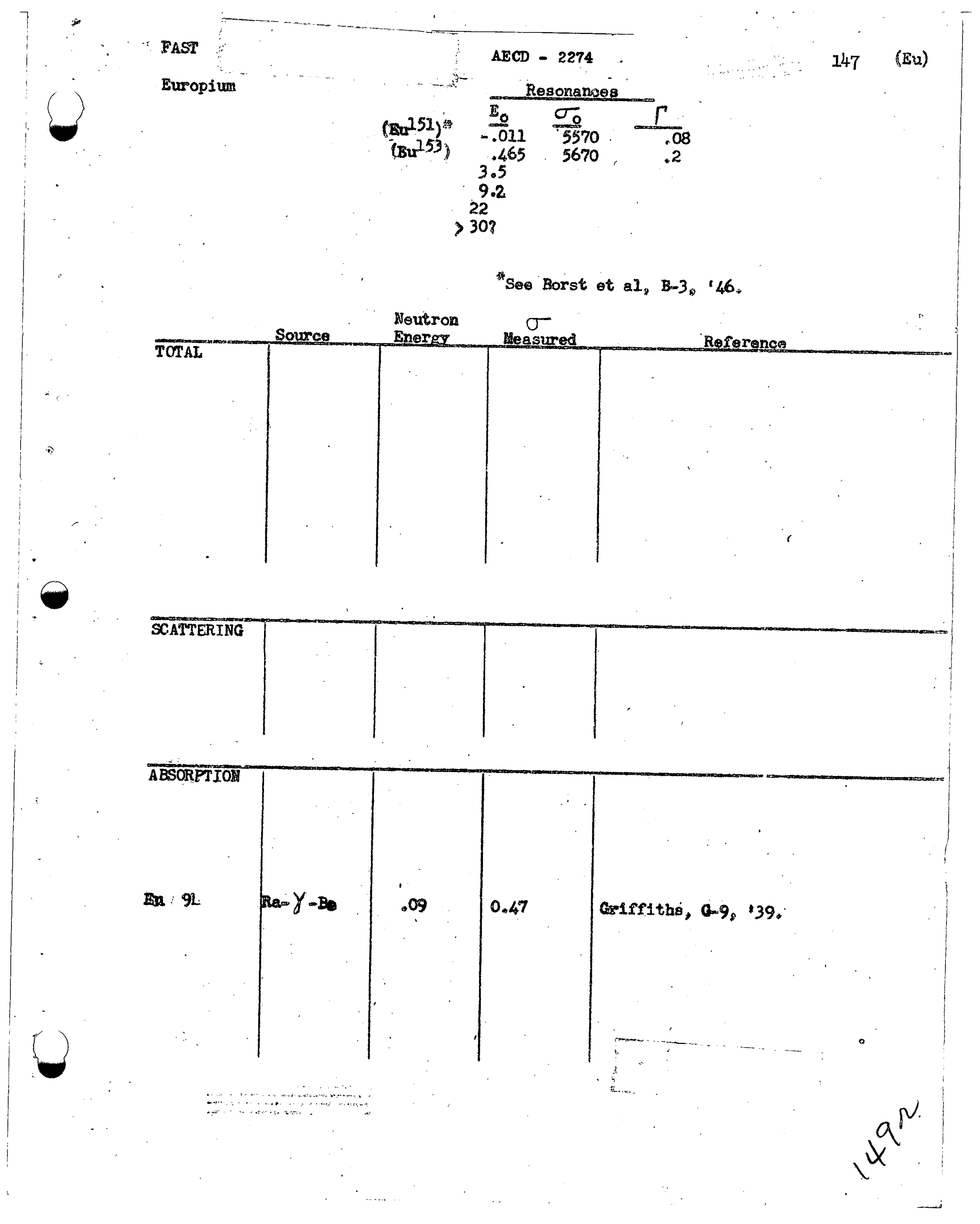




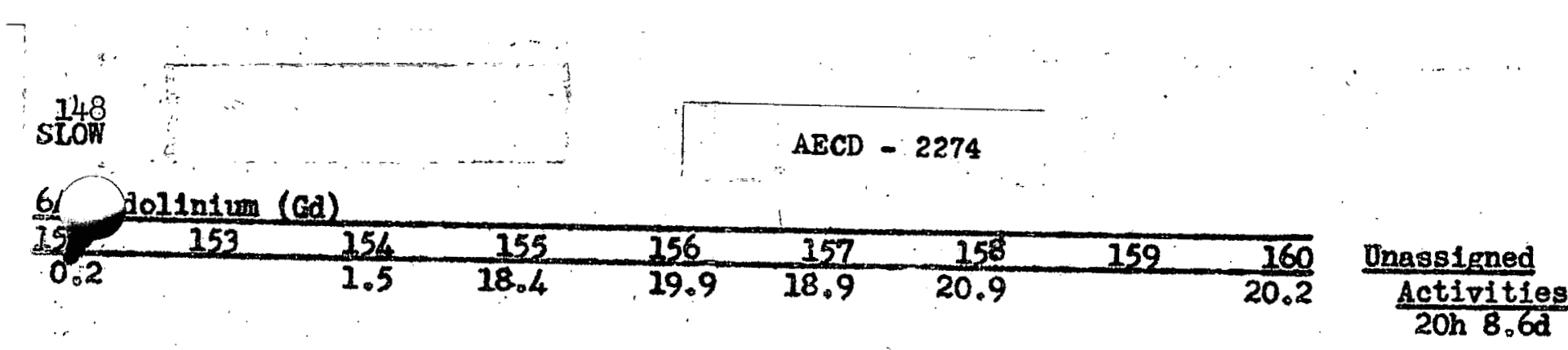

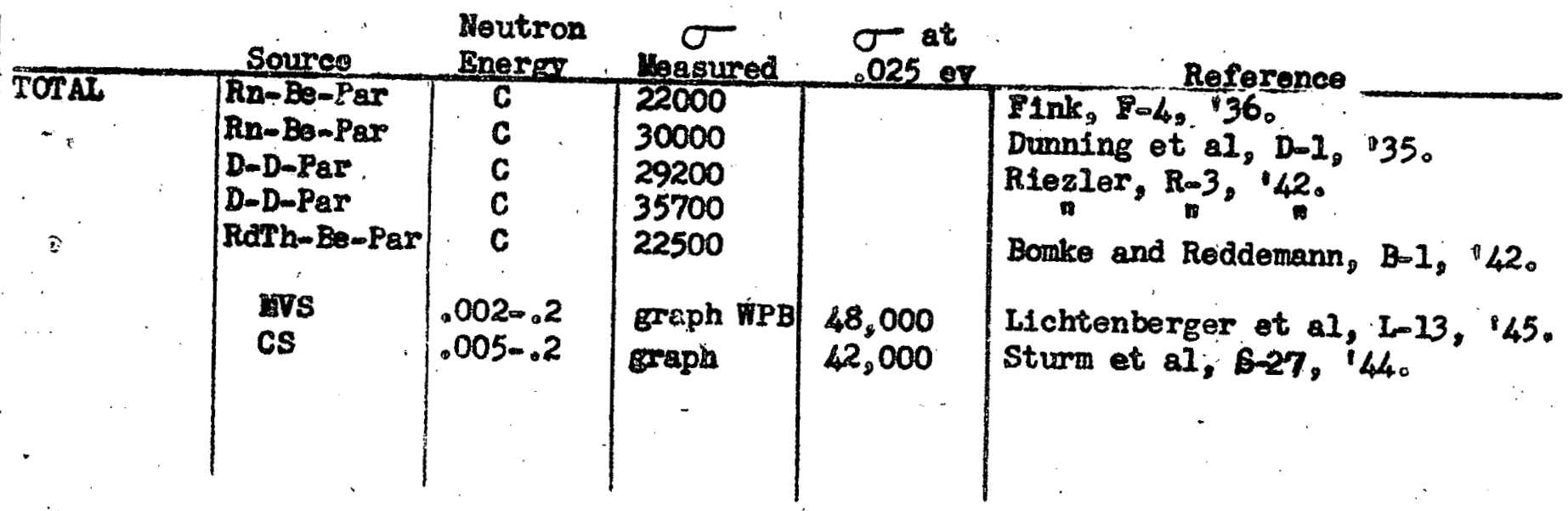
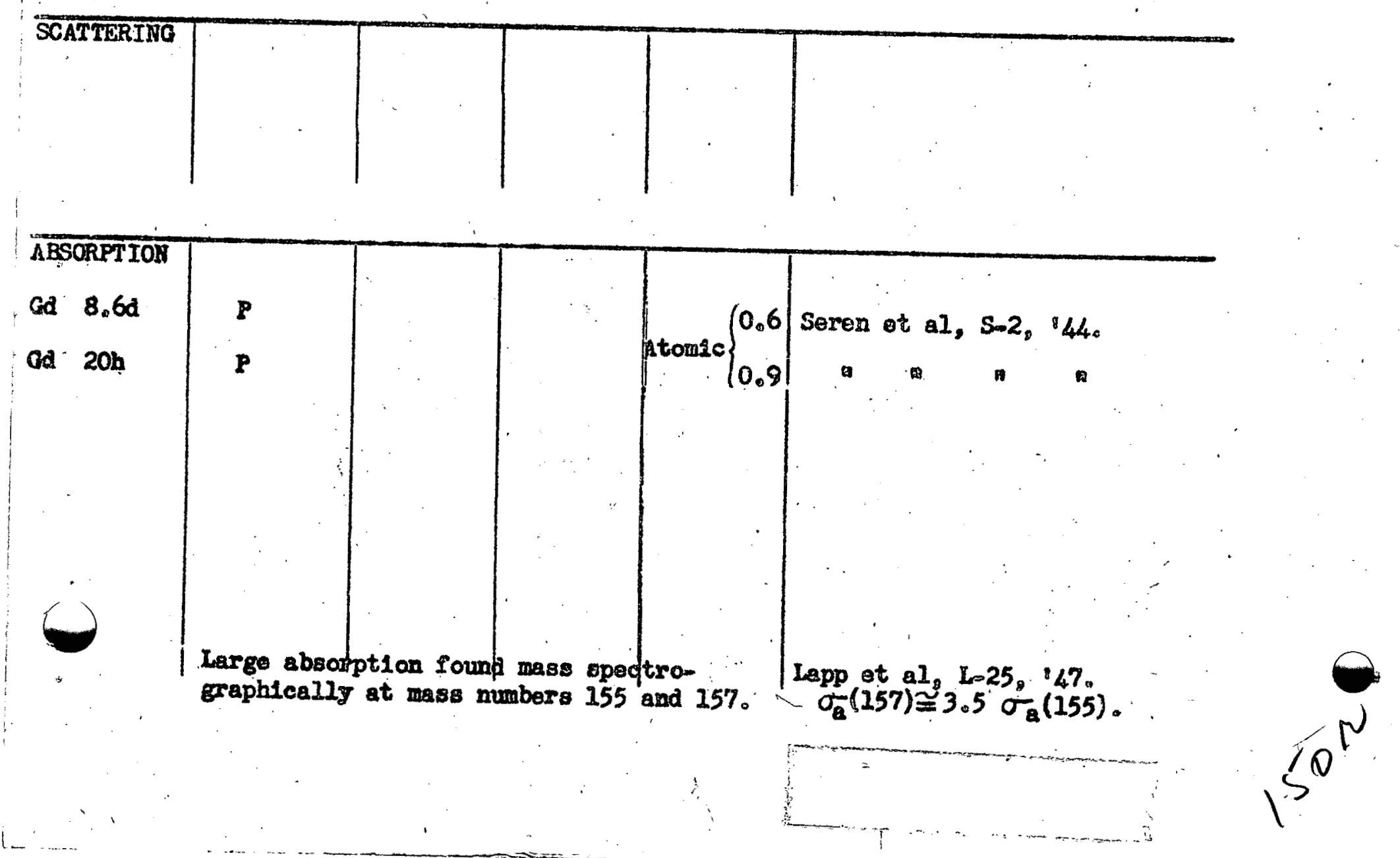


\section{TAST}

Gadolinium

AECD - 2274

$0.044 \quad 32,000 \quad .10 \mathrm{CS}$

$0.028 \quad 45,000 \quad .236$ RivS

จ
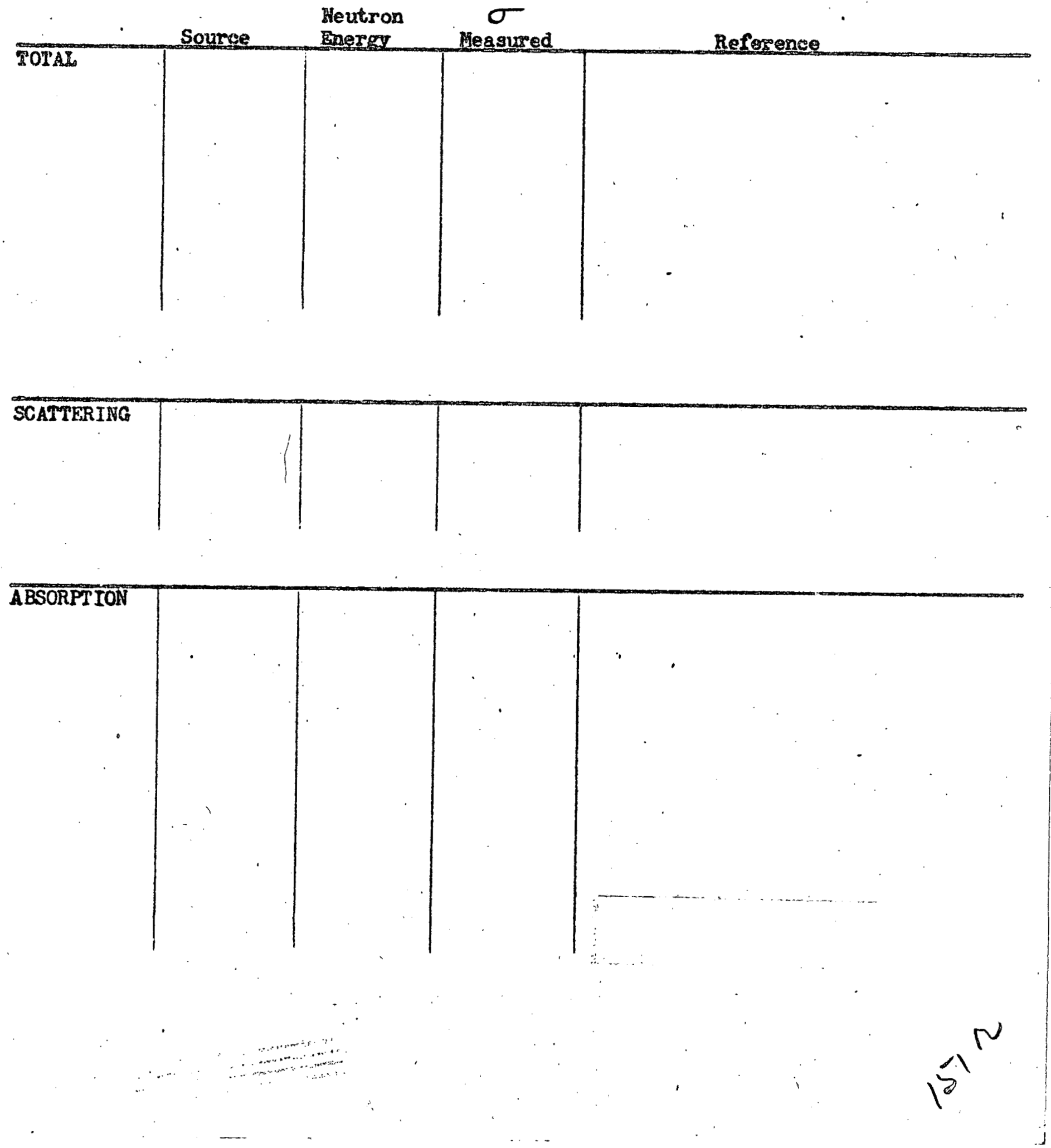

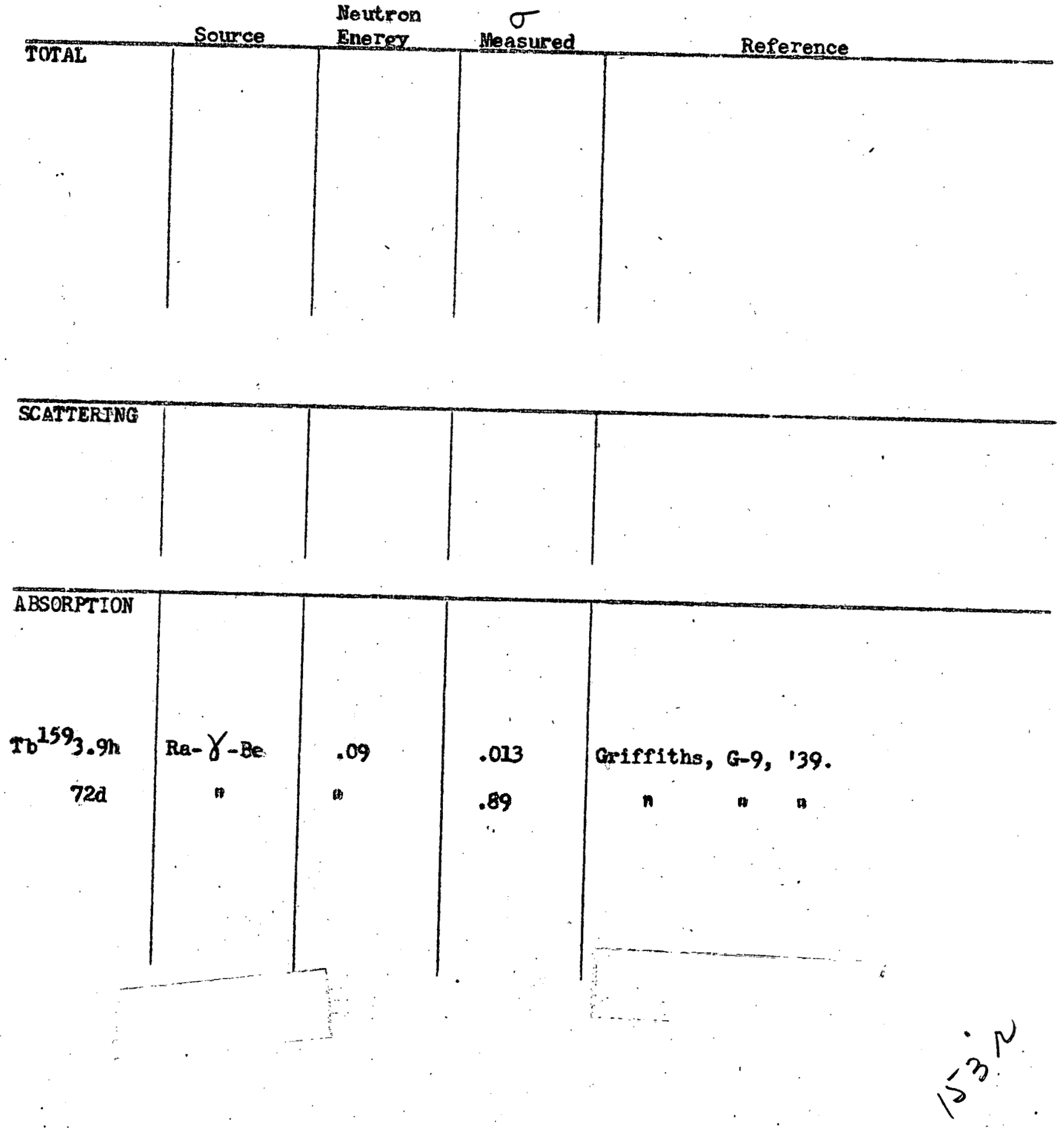
6 Joprosium (Dx)

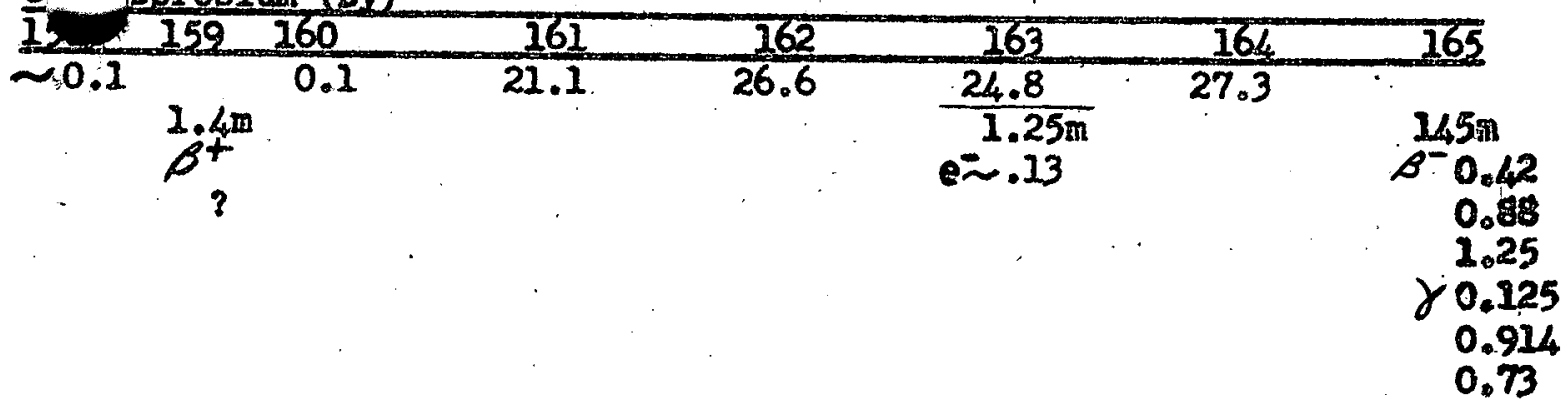

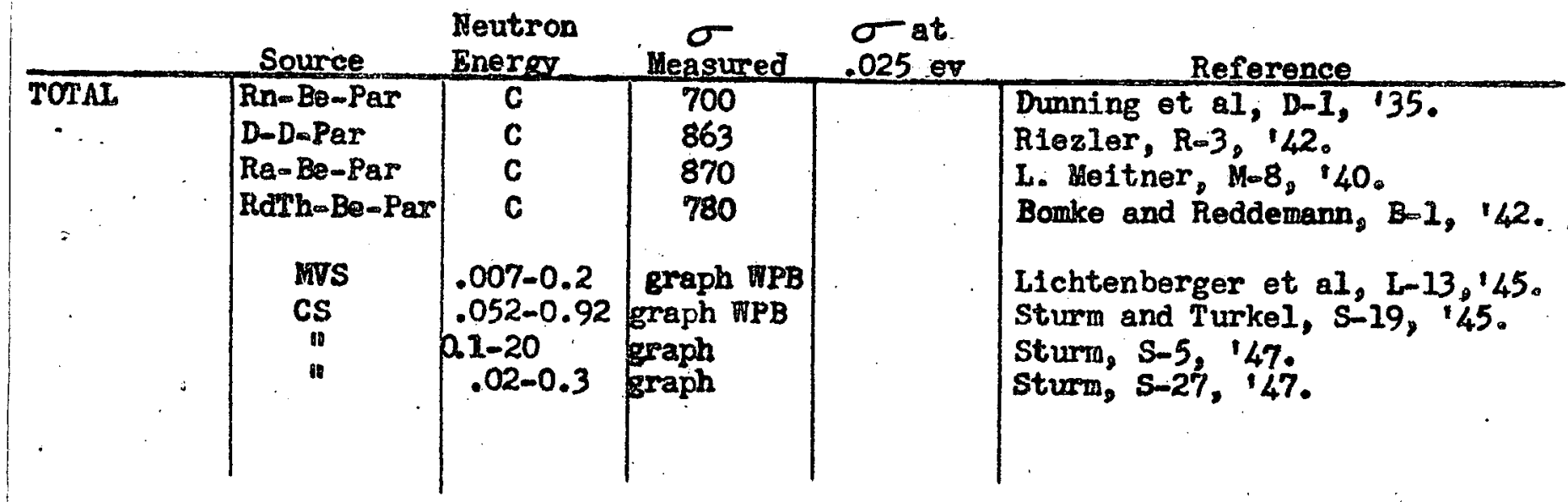

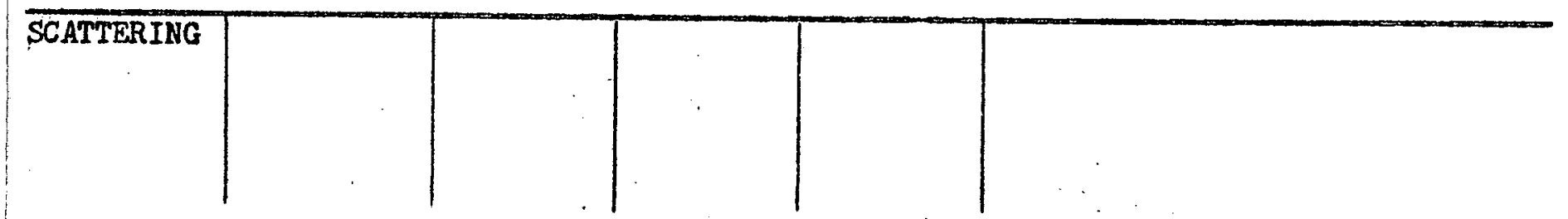

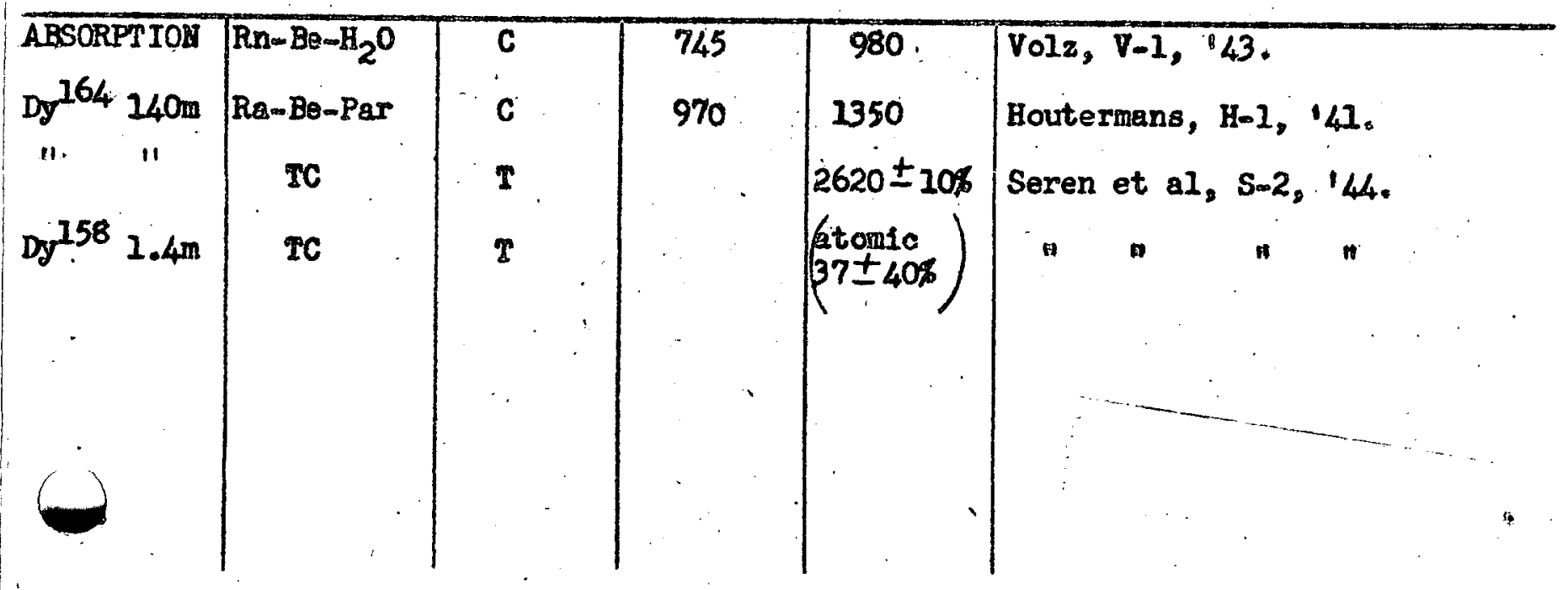


FAST

ABCD - 2274

153

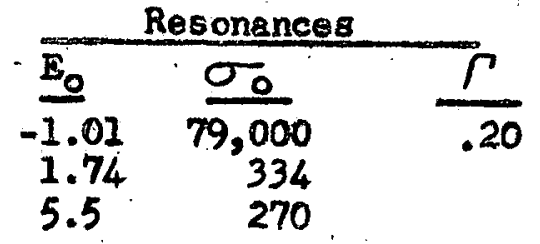
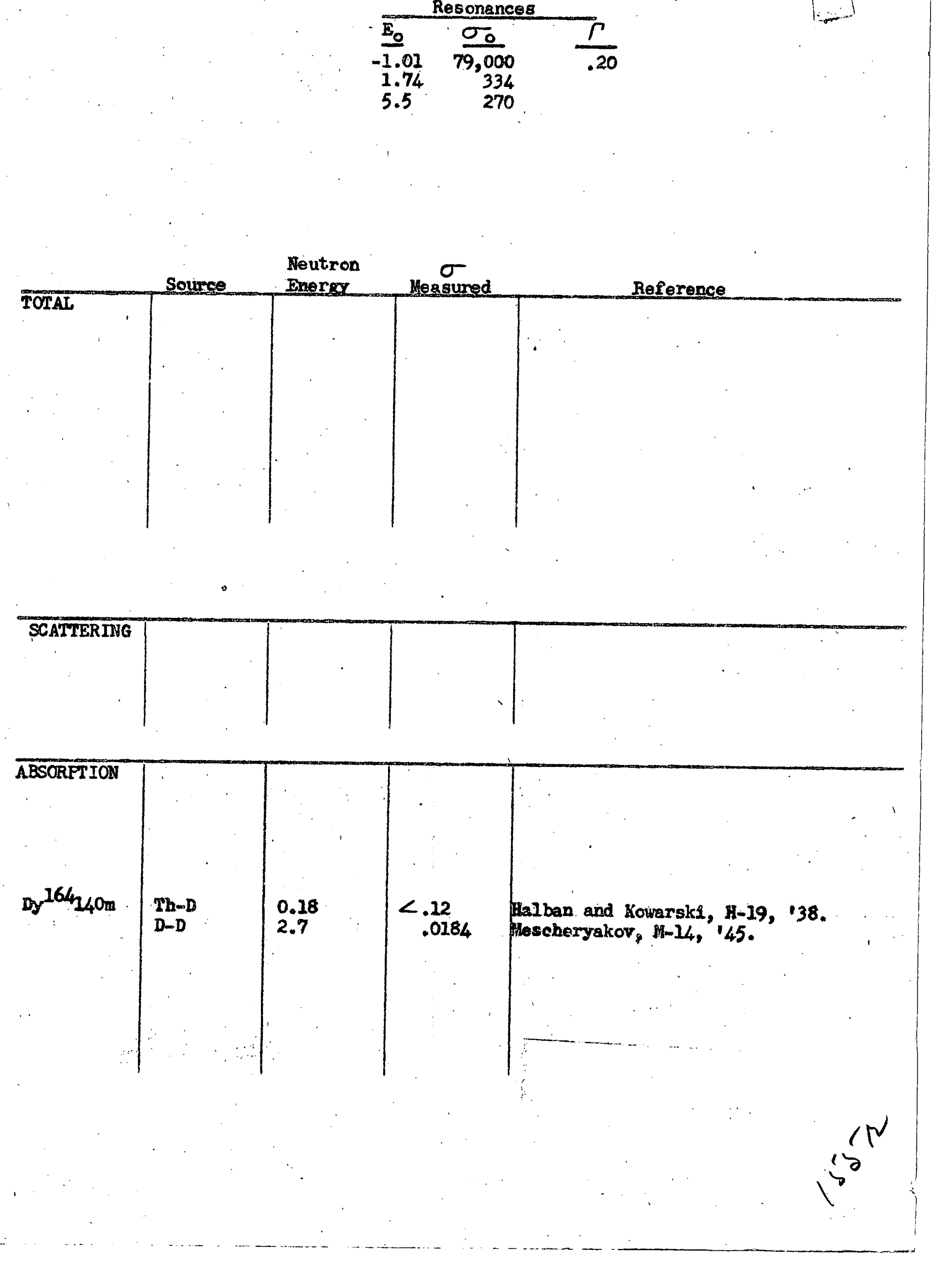
$6 \%$ Imium (Bo)

$\frac{6}{16}-\frac{165}{100}$

166

$\begin{array}{cc}47 \mathrm{~m} & 27 \mathrm{~h} \\ \beta^{-} & \beta^{-1.9}\end{array}$
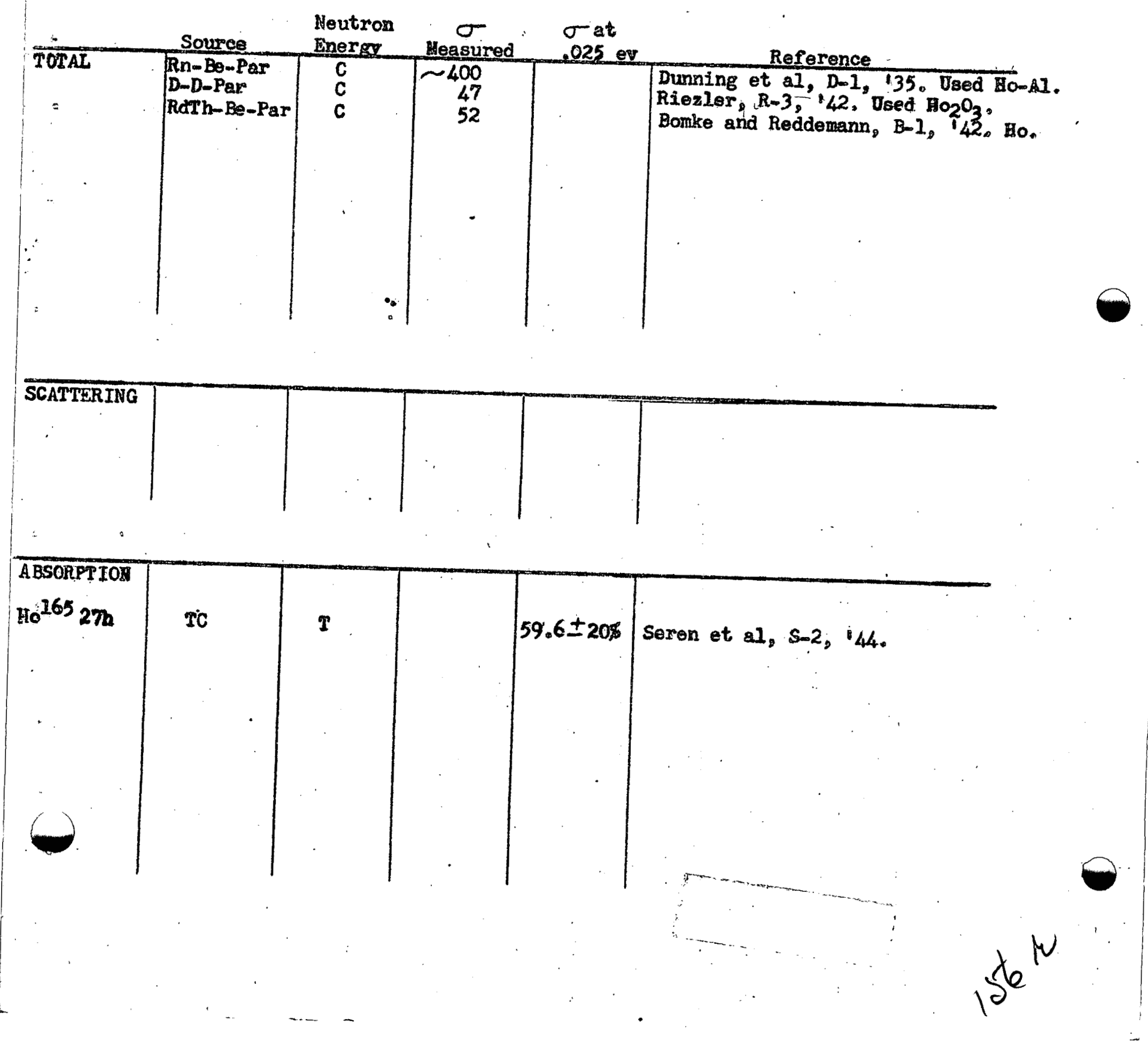

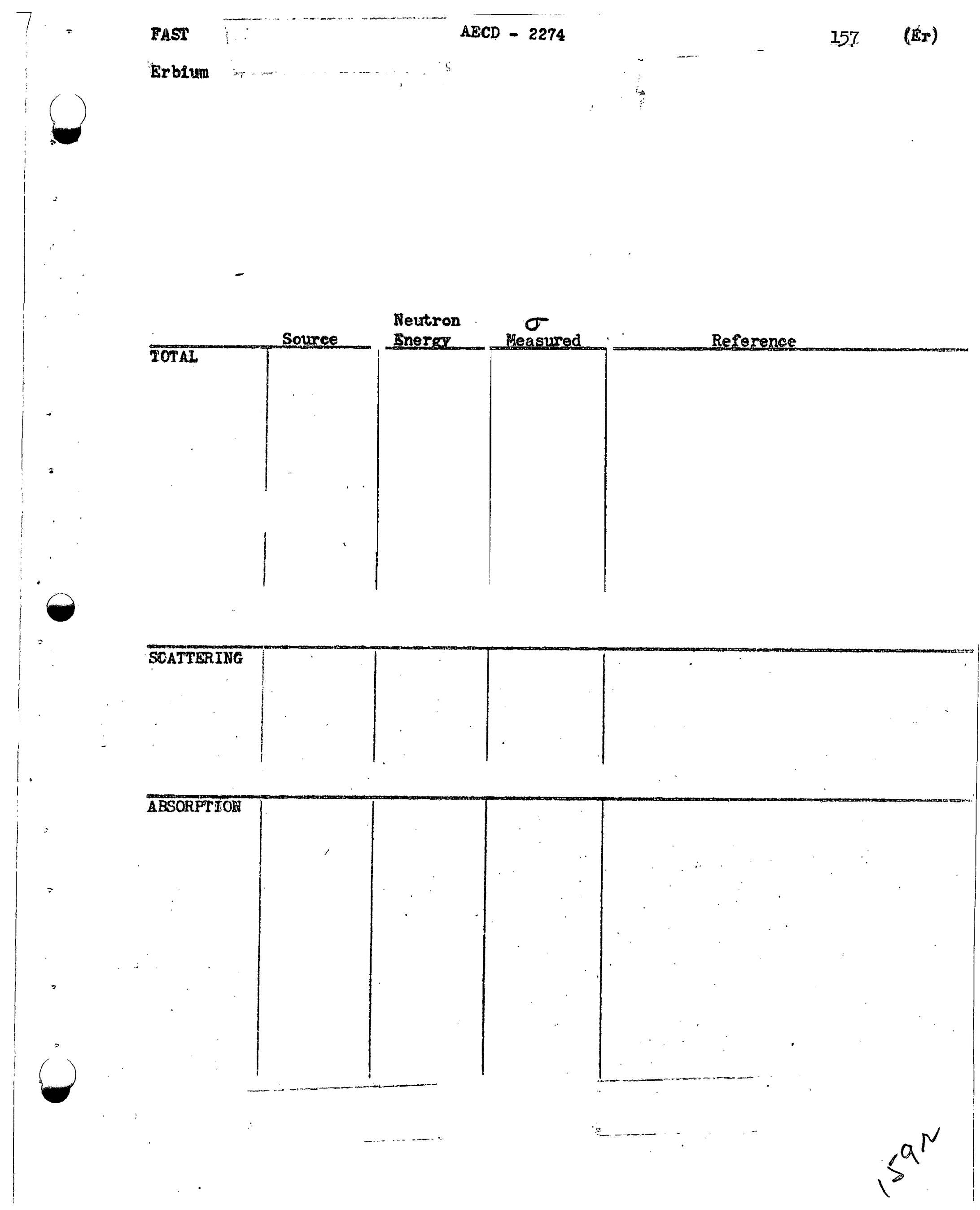


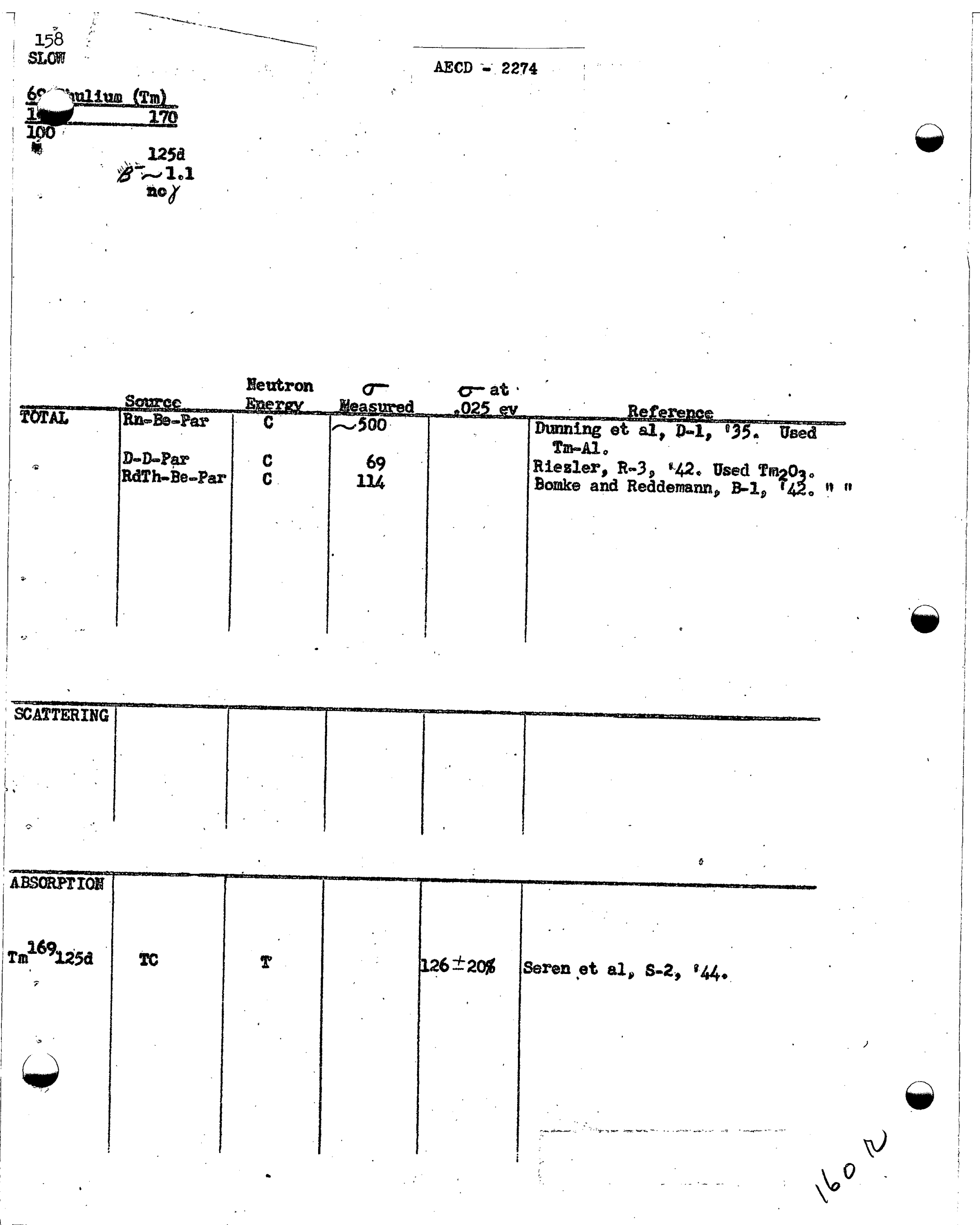


FAST

AECD - 2274

159. (Tm)

Thulfun
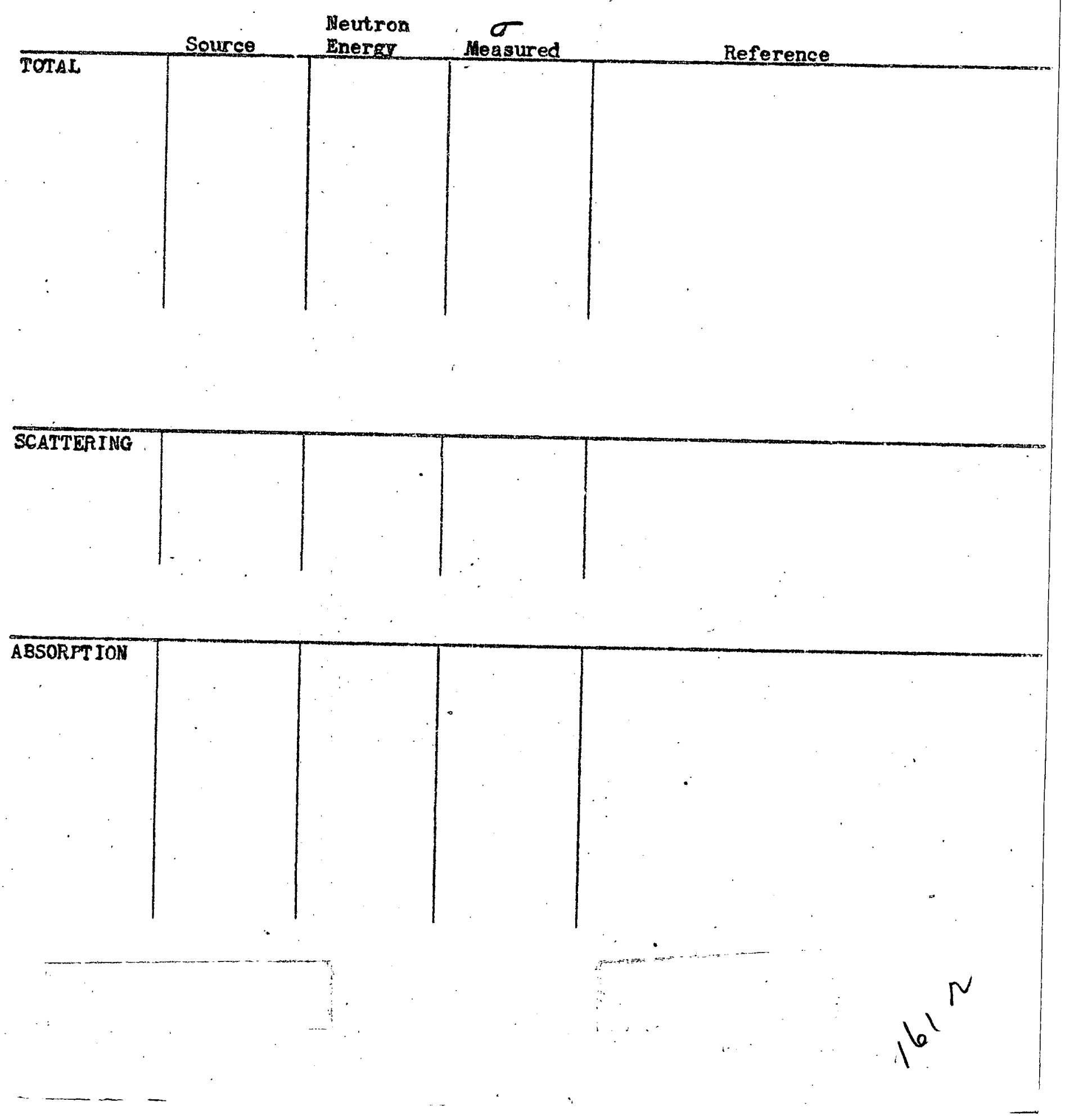


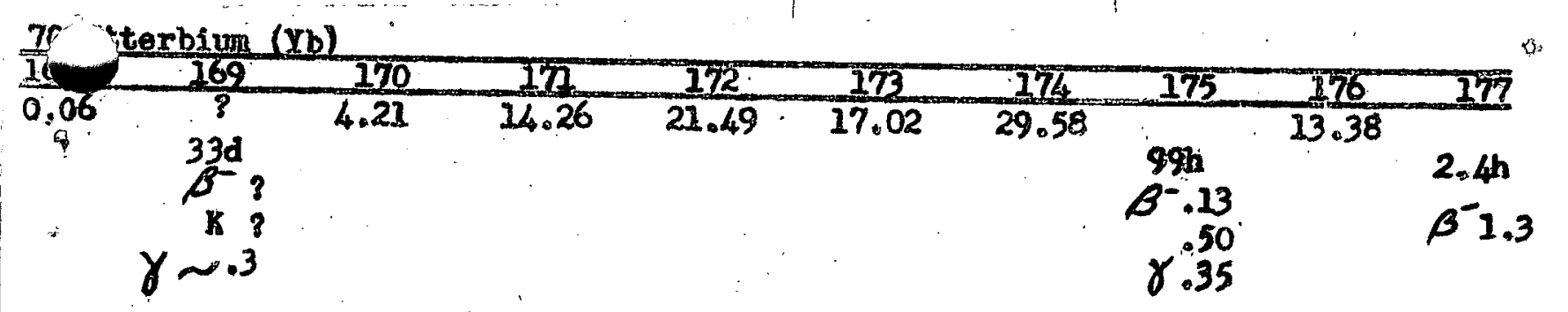
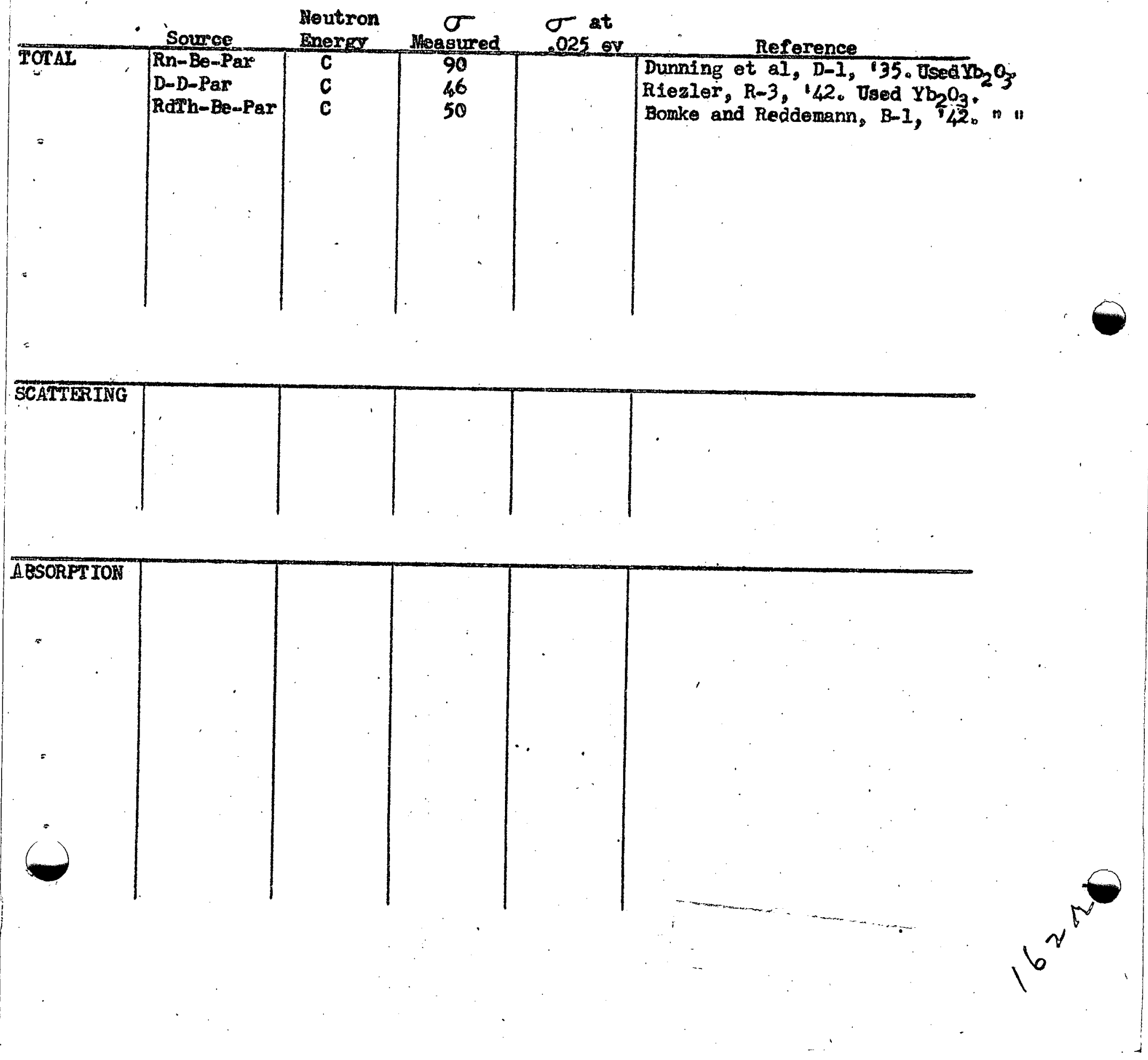


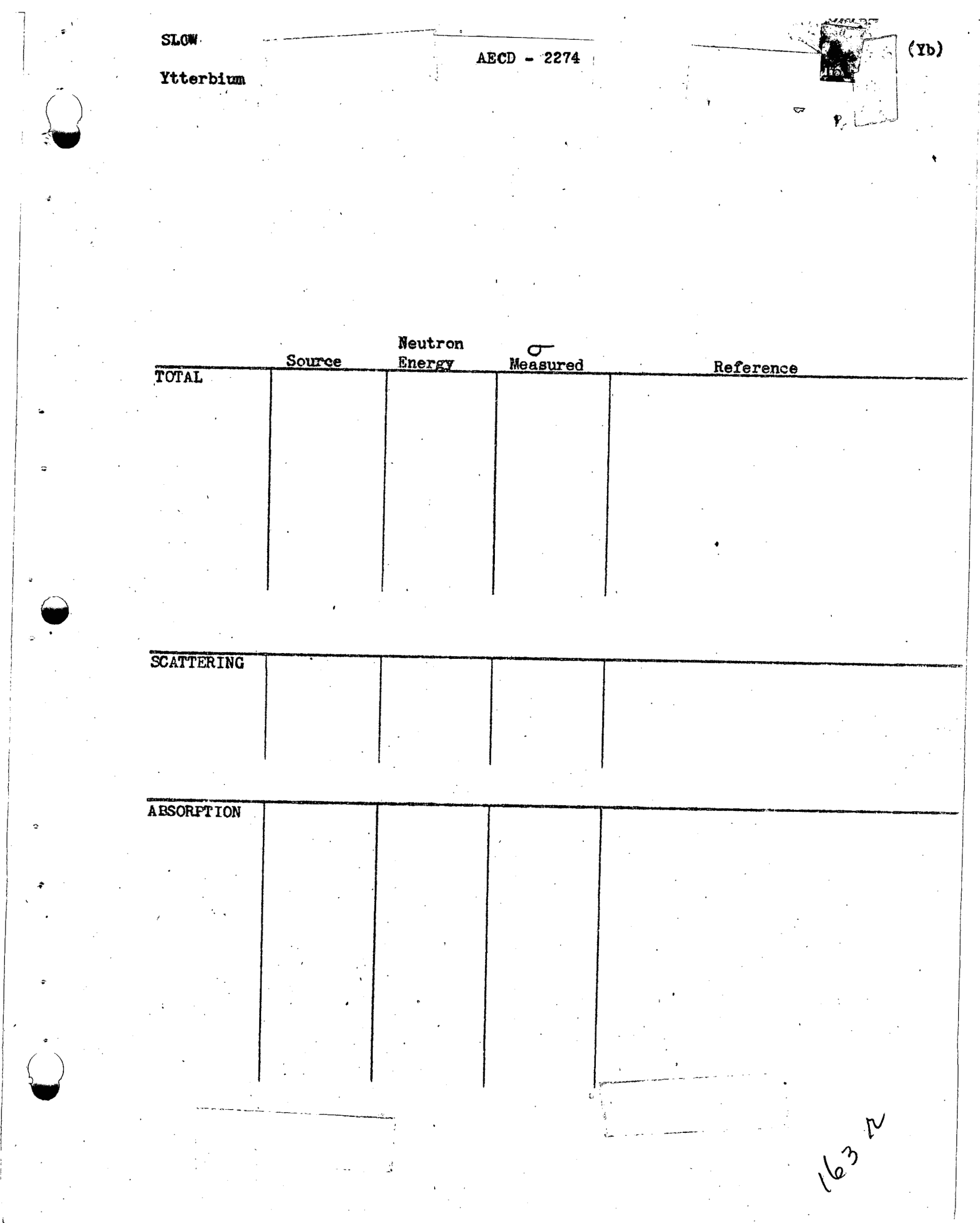



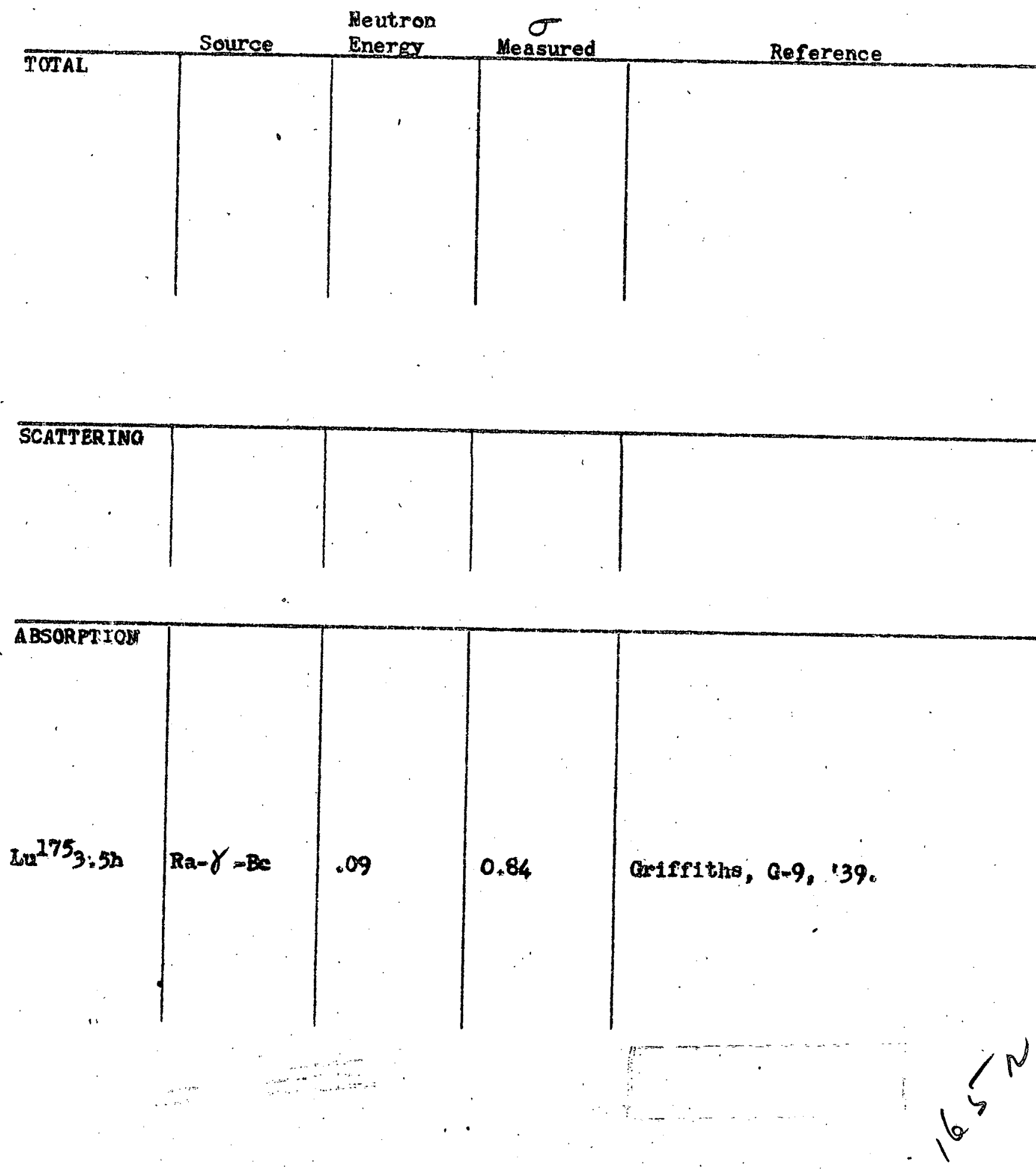


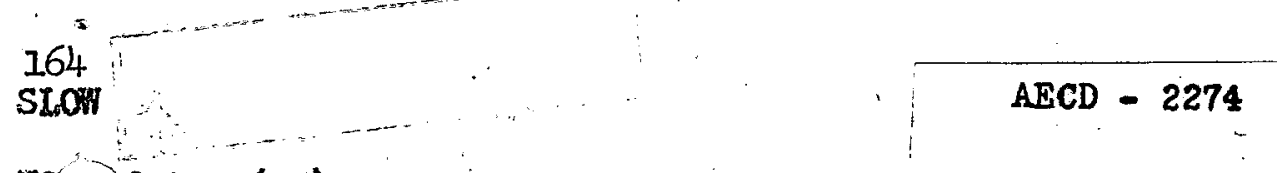

77 onfun (BS)

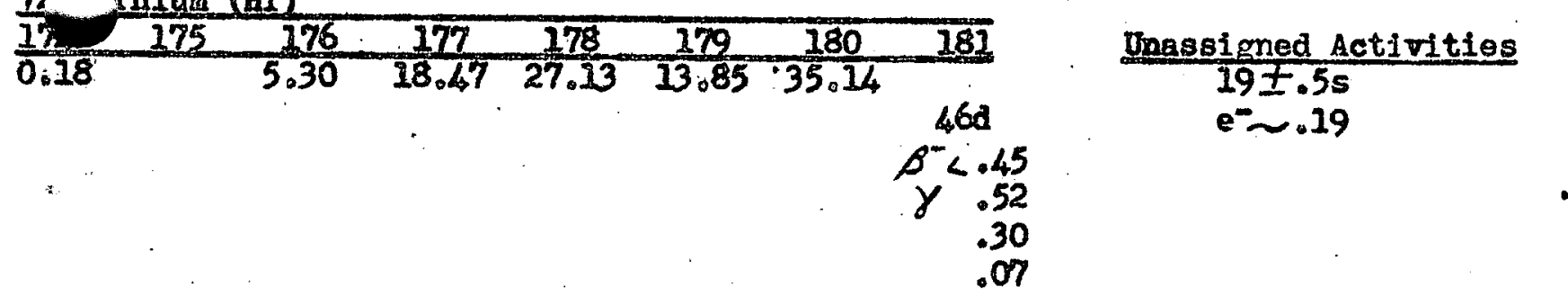
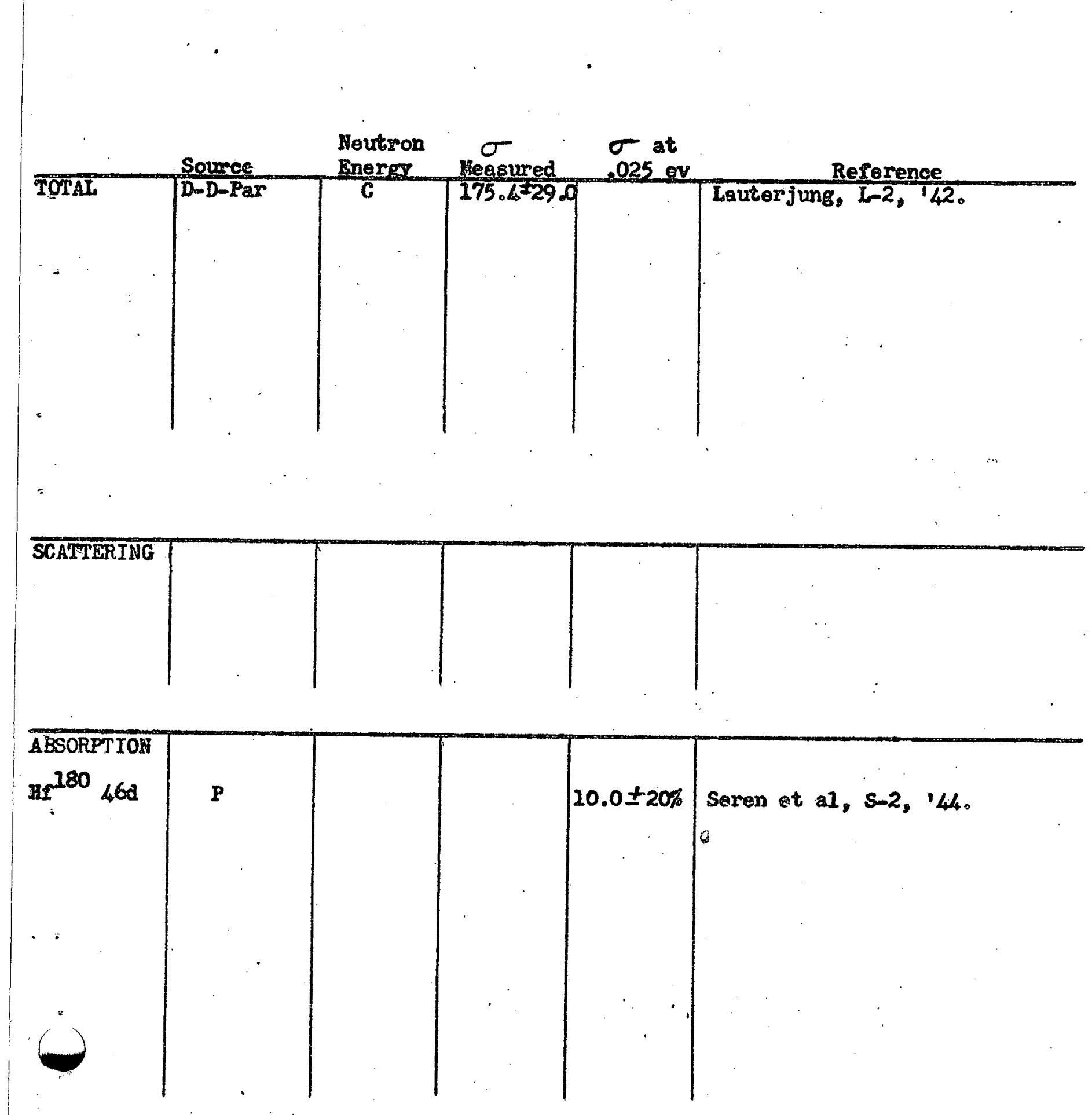


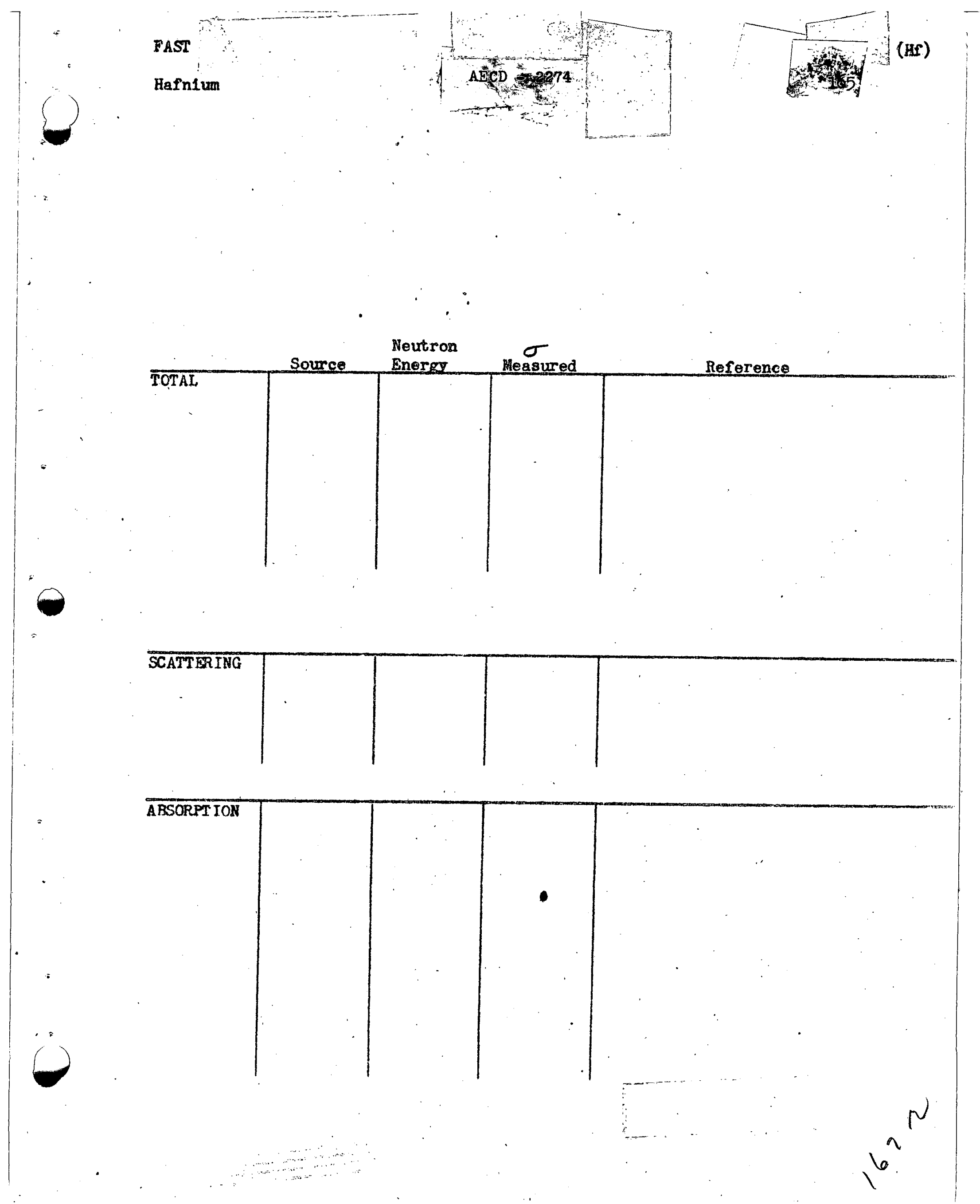




\section{Tantalun (Ta)}

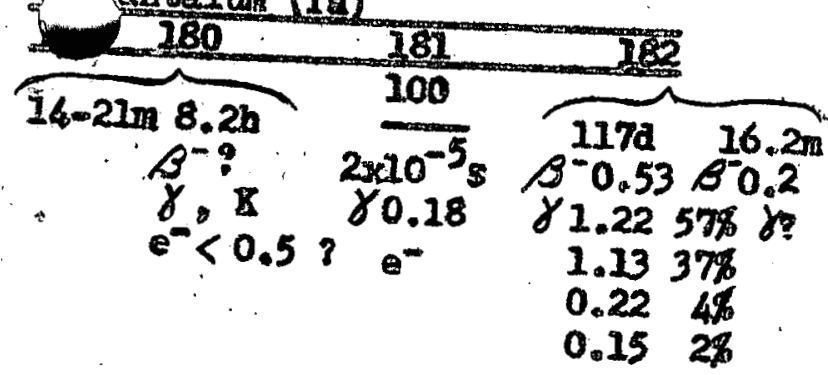

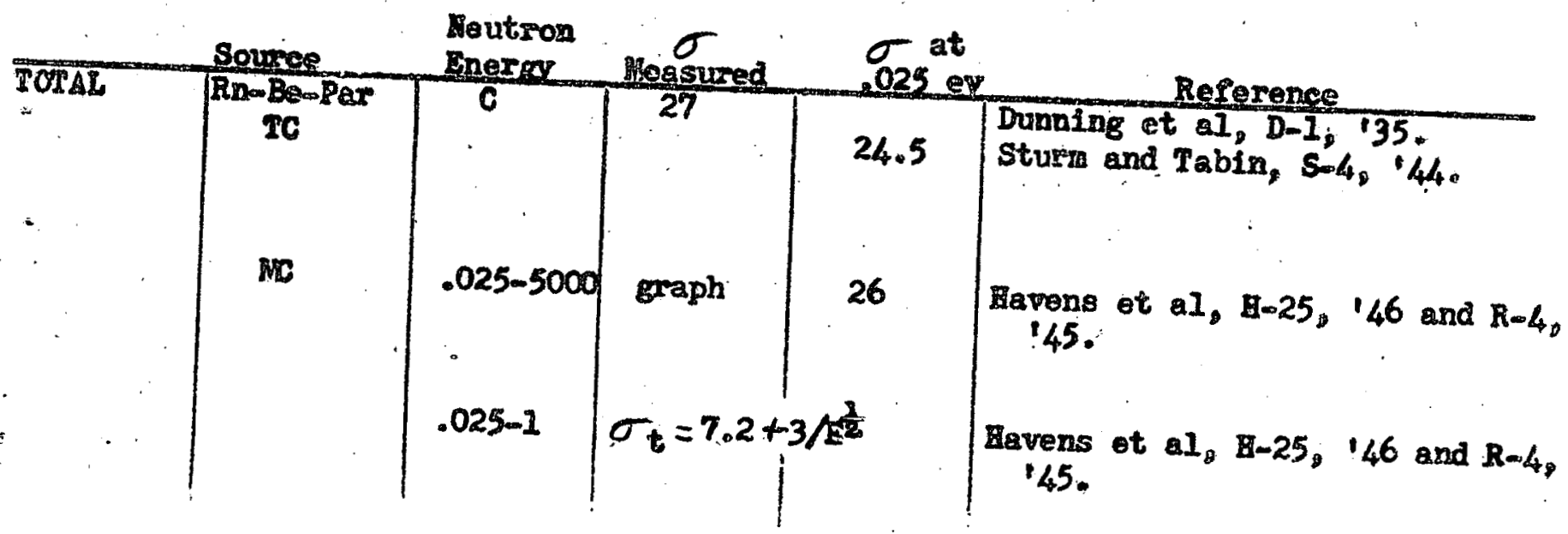

\begin{tabular}{|c|c|c|c|c|}
\hline SCATTERTII & $\begin{array}{l}\text { RasBe }- \text { Par } \\
T C \\
T C\end{array}$ & $\bar{c}$ & $\begin{array}{c}-10 \\
4.83 \\
4.9 \\
4.9\end{array}$ & 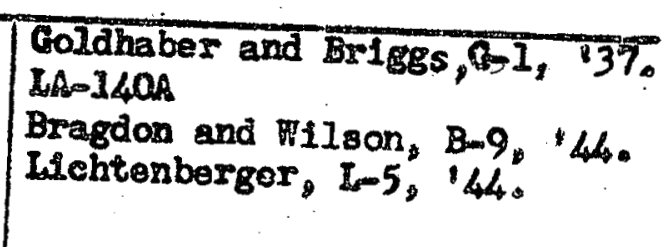 \\
\hline
\end{tabular}

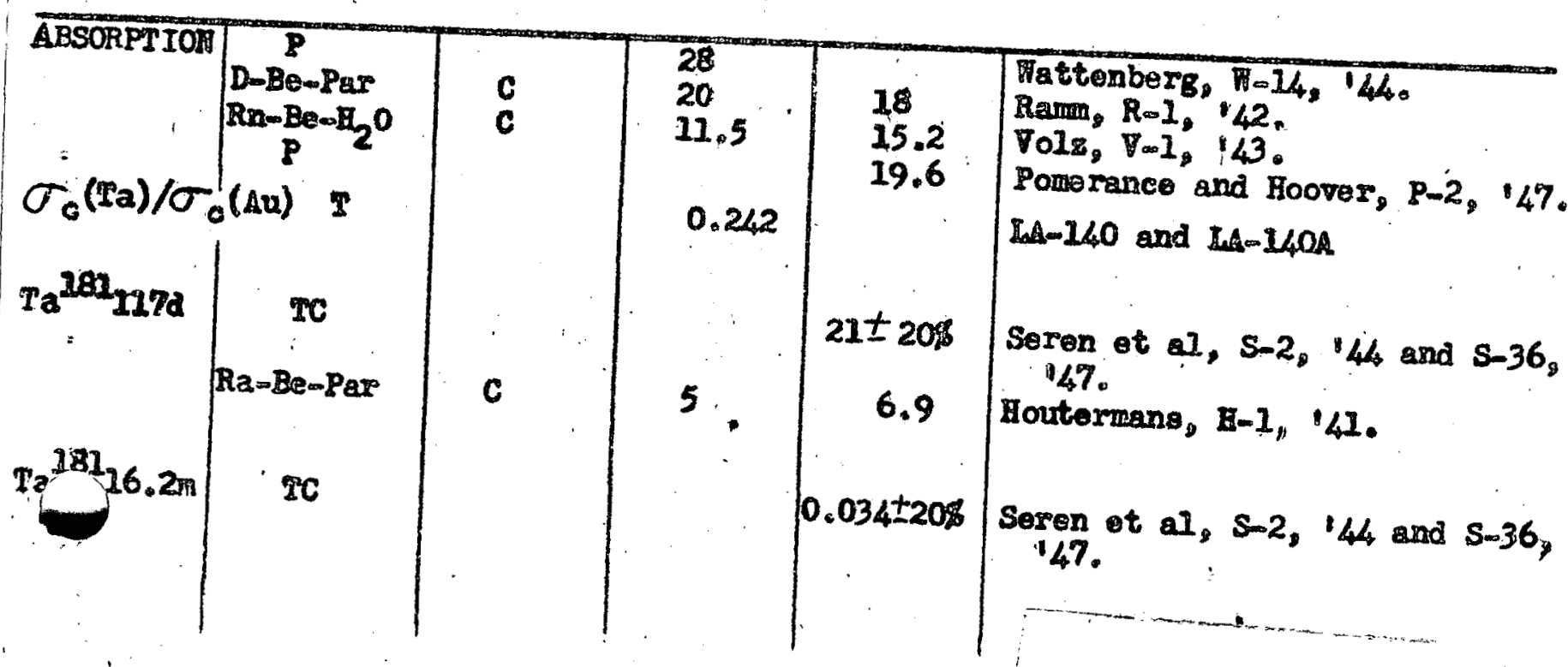




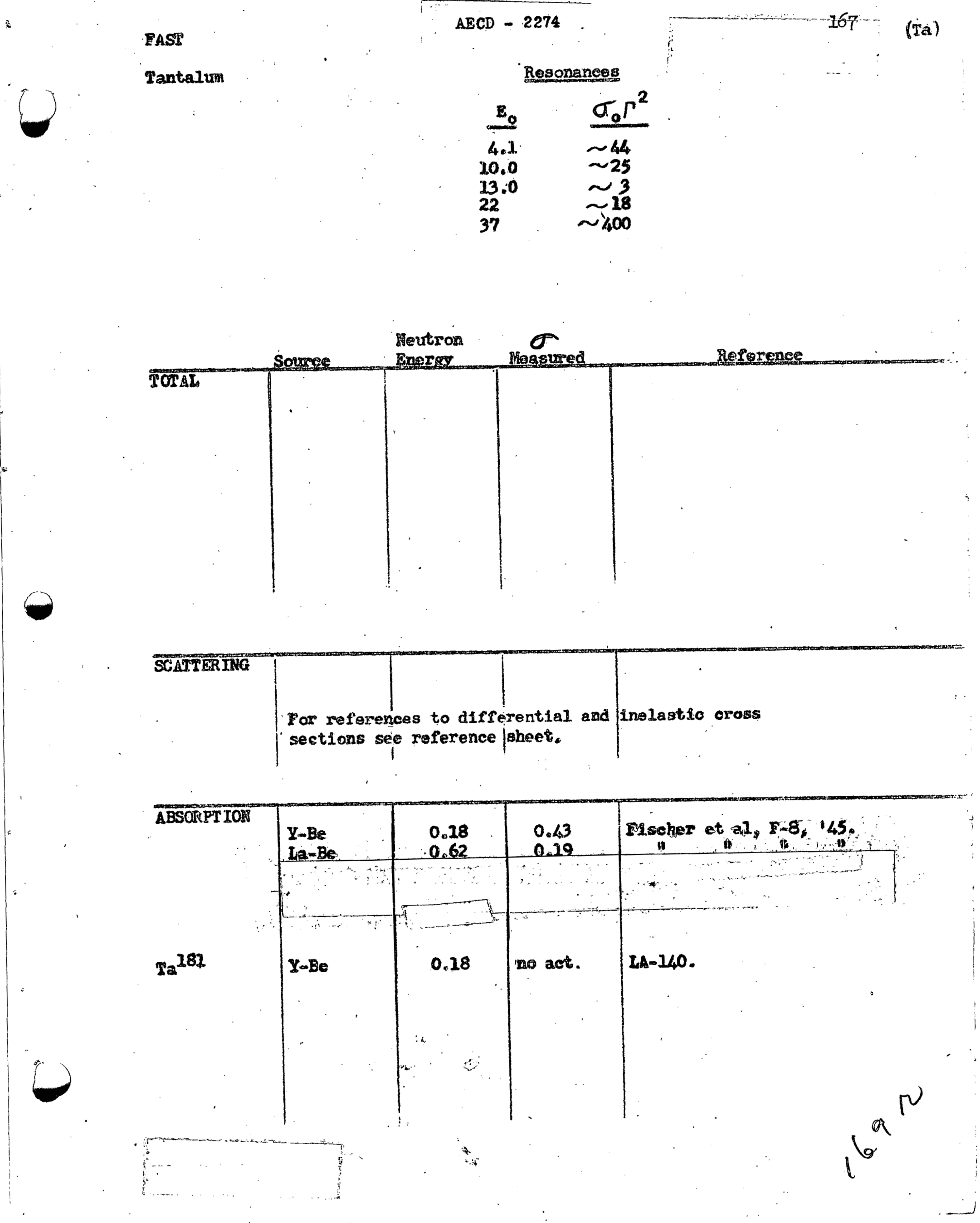




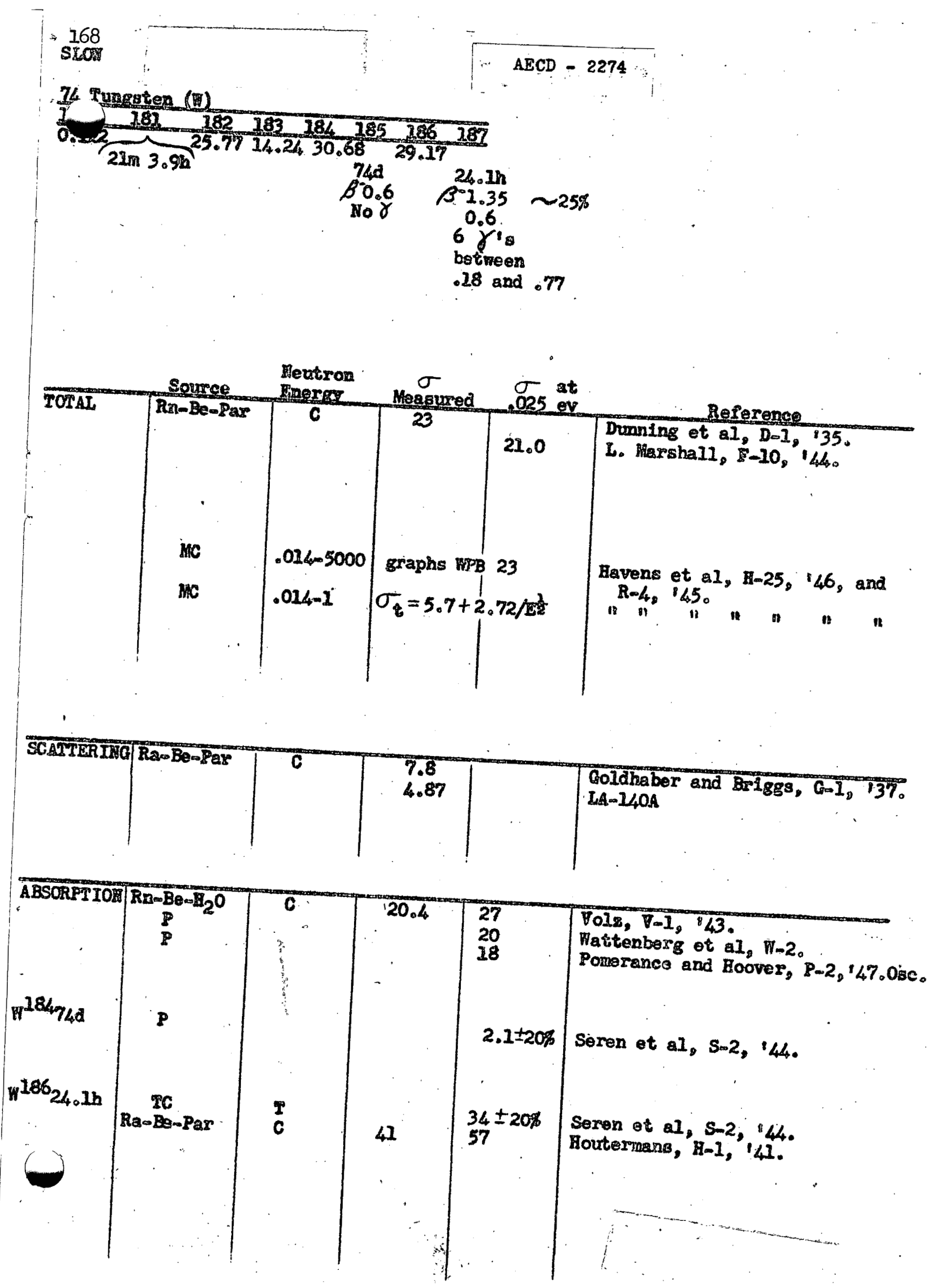


Tungsten

Resonances

$\begin{array}{rr}\text { Eo }_{0} & \sigma_{0} \Gamma^{2} \\ 4.0 & \sim 13 \\ 7.4 & \sim 5 \\ 18 & \sim 3000 \\ 45 & \sim \tilde{H}_{400} \\ 180 & \sim 10,000 \\ 1100(?) & \end{array}$

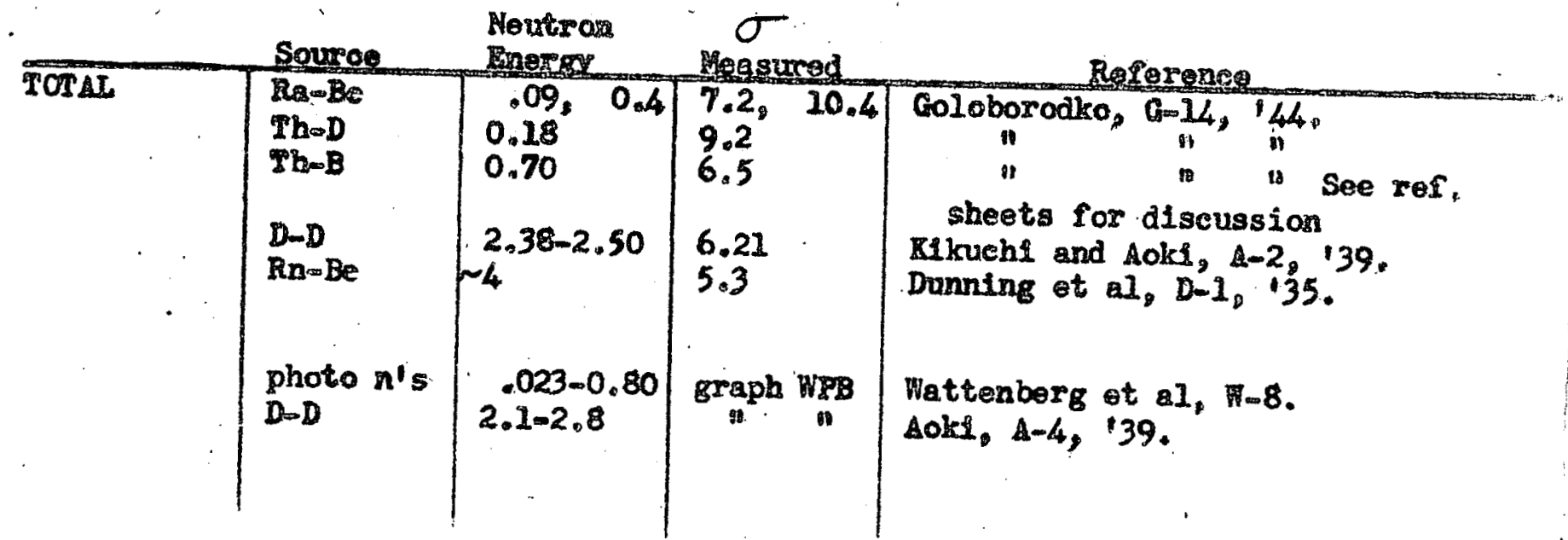

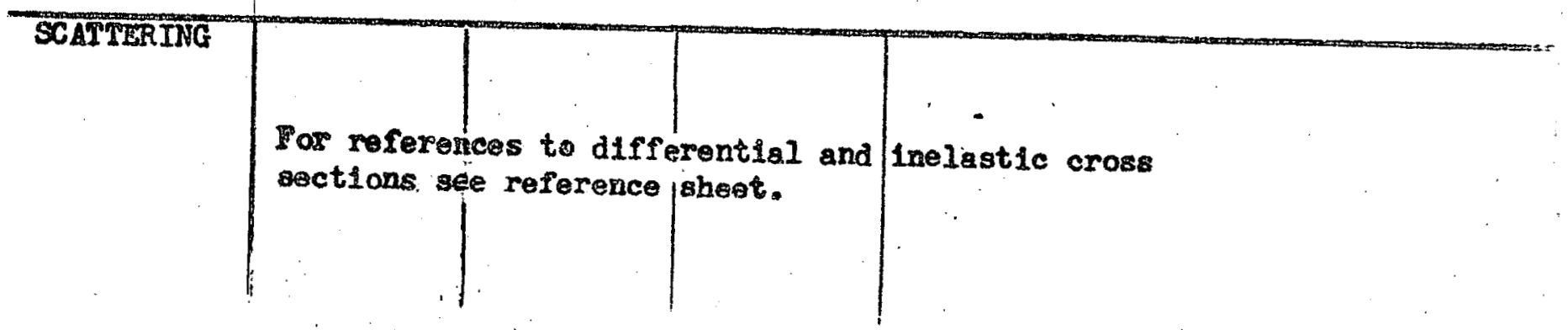

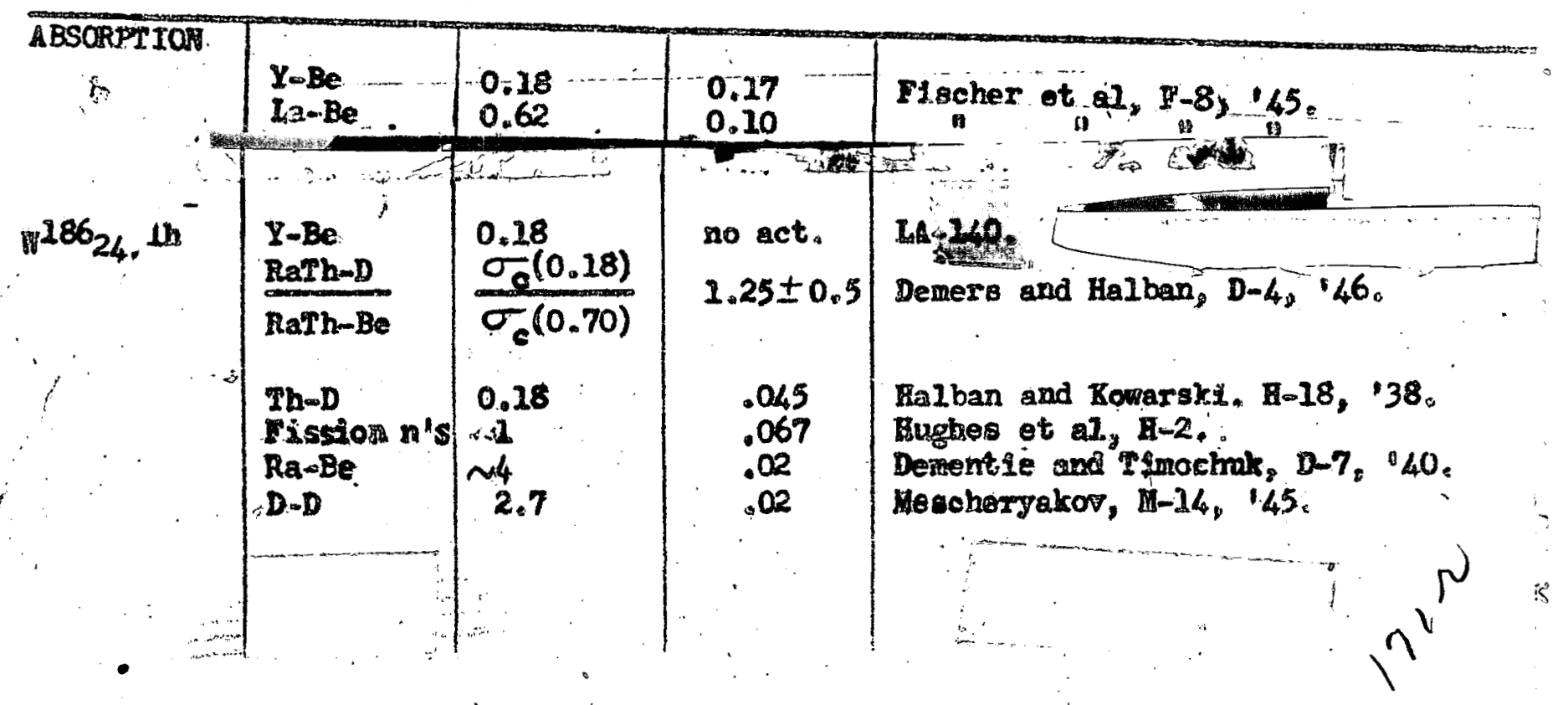




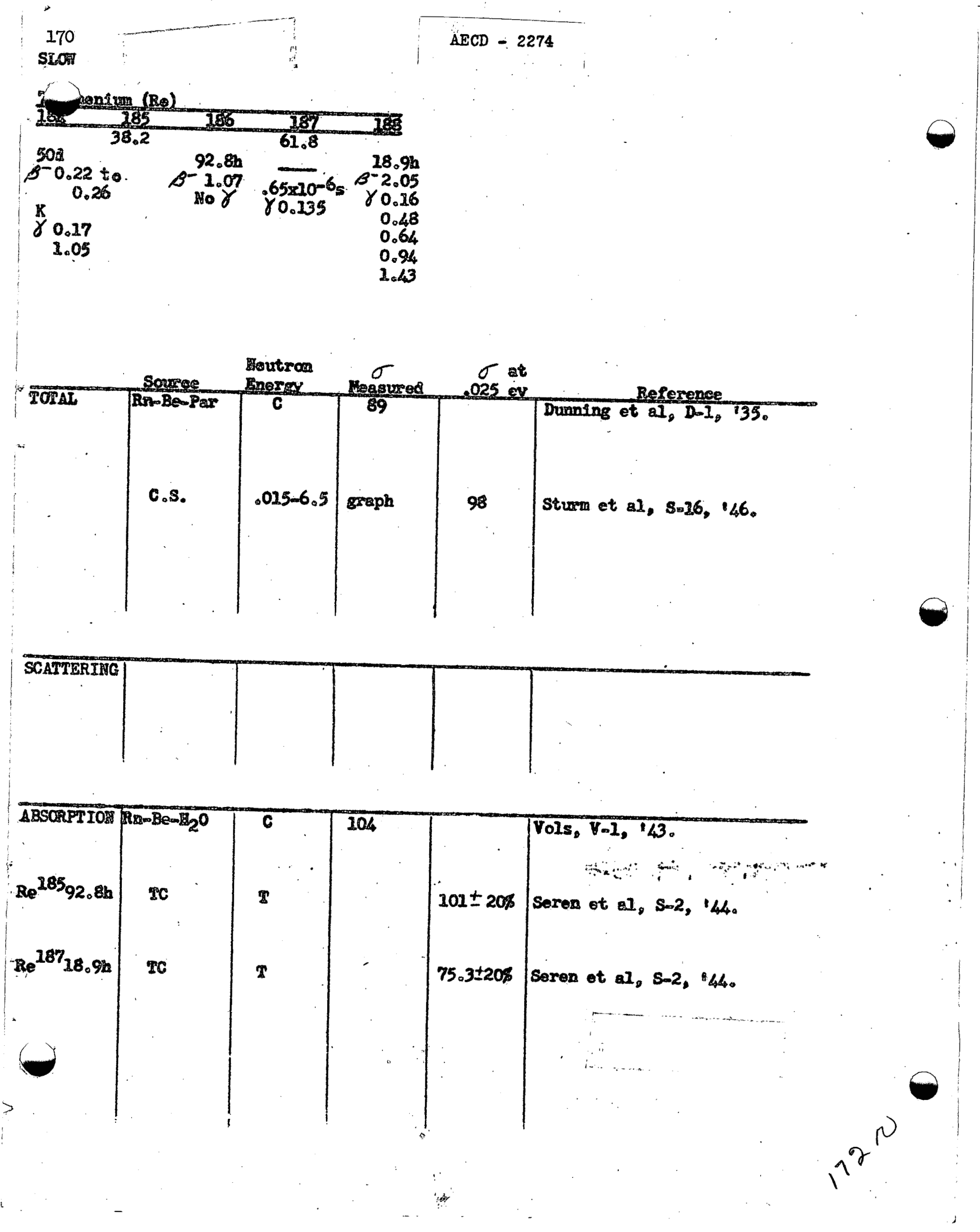




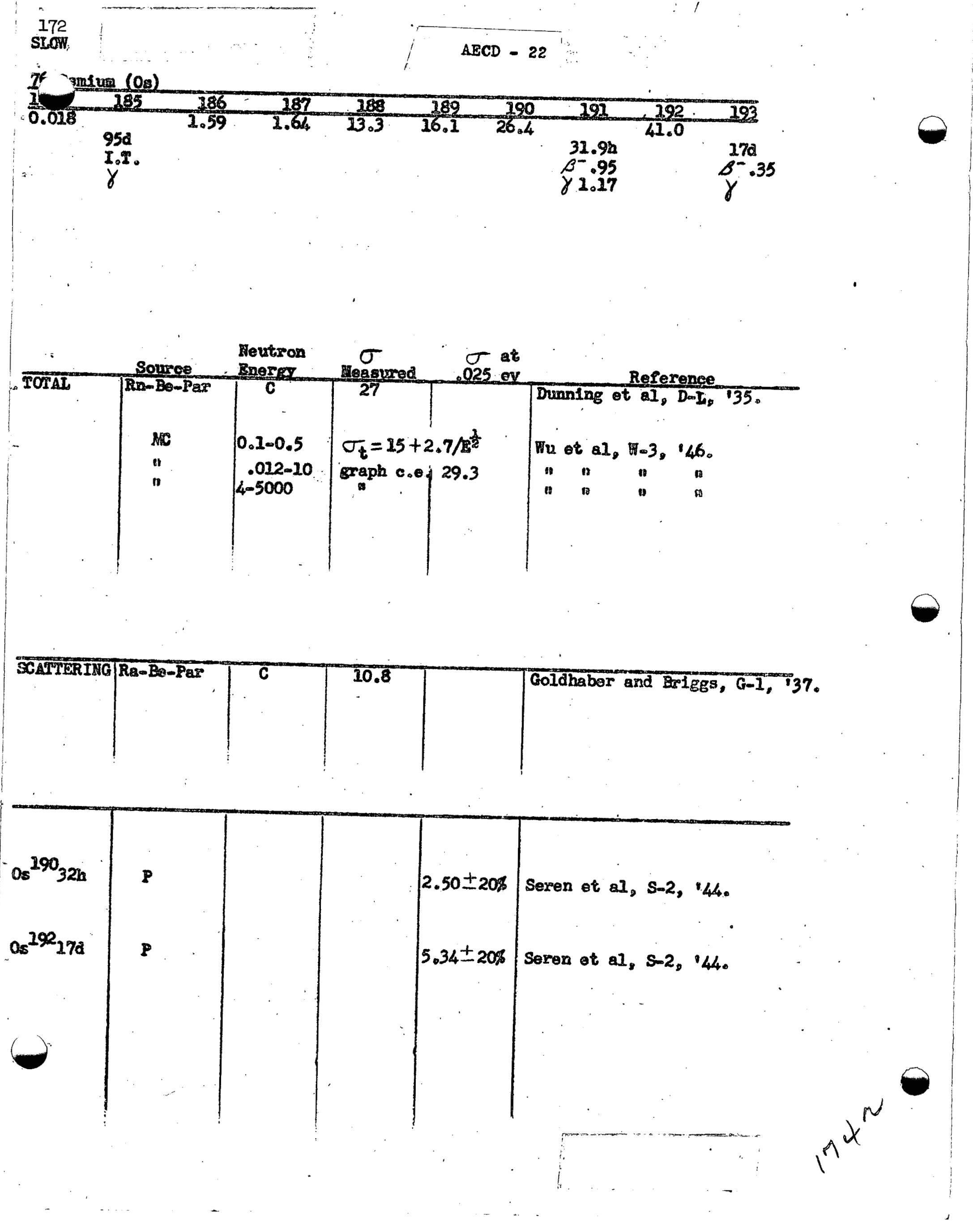




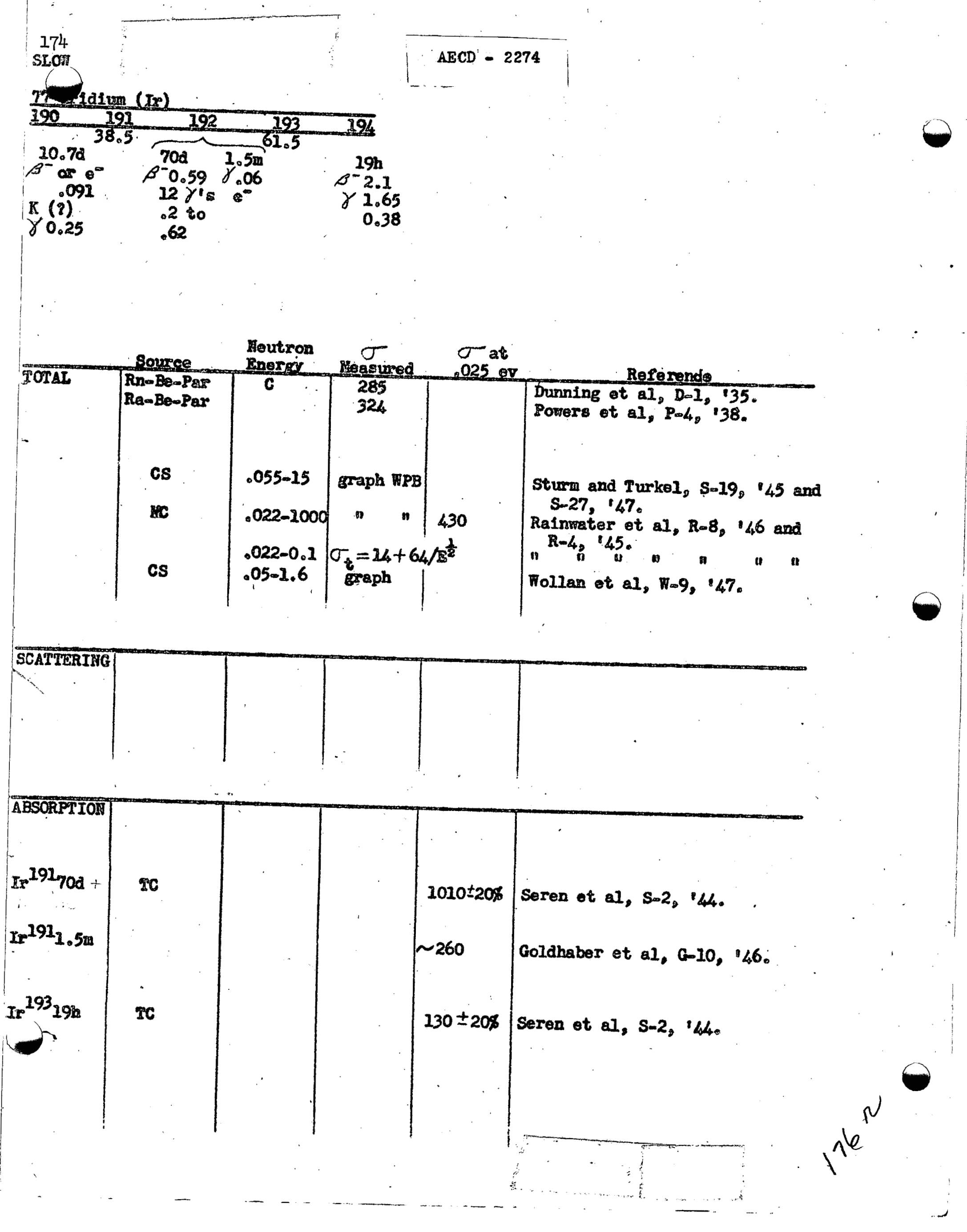




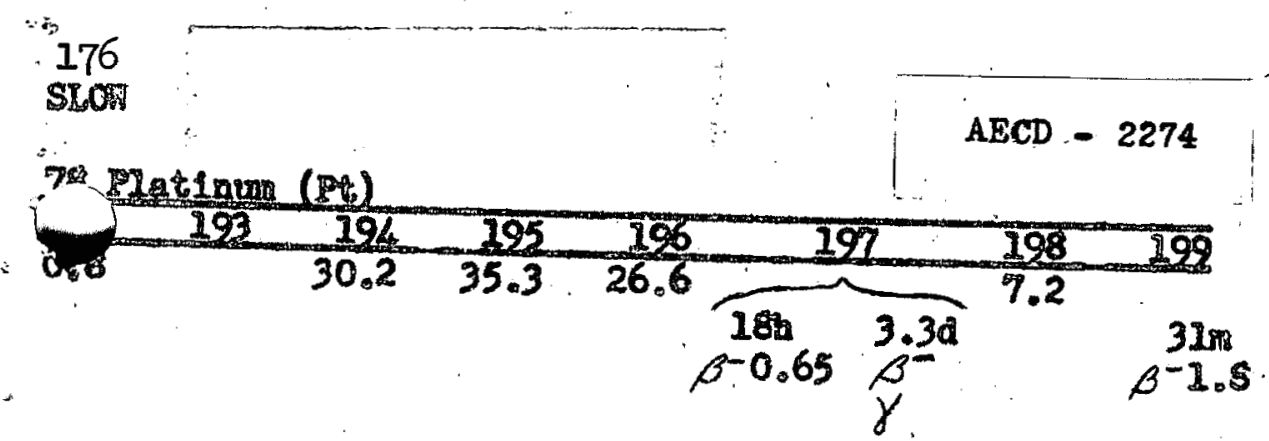

Daasaigned Activity $80 \mathrm{~m}$

$e^{*}$ ?
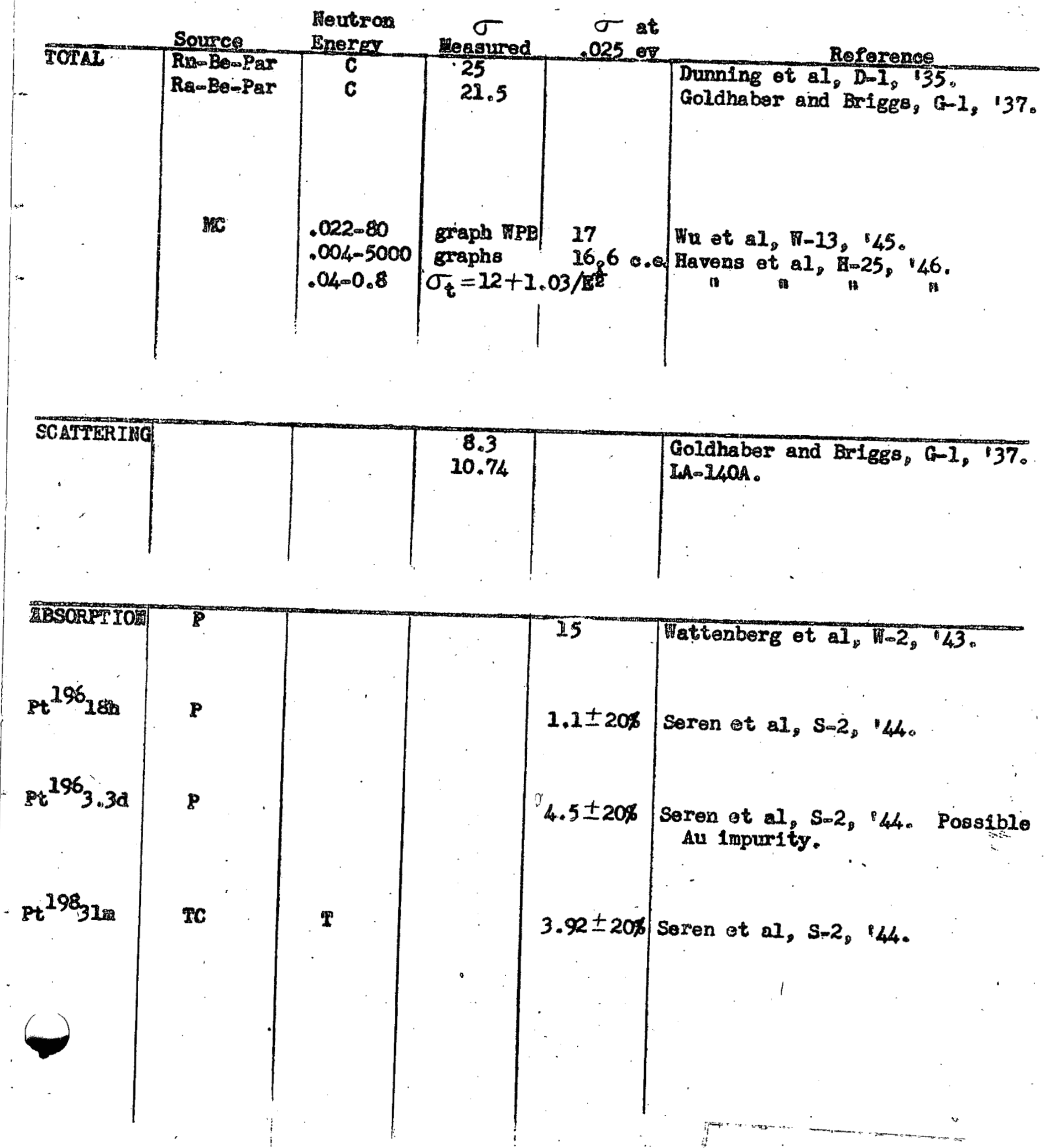

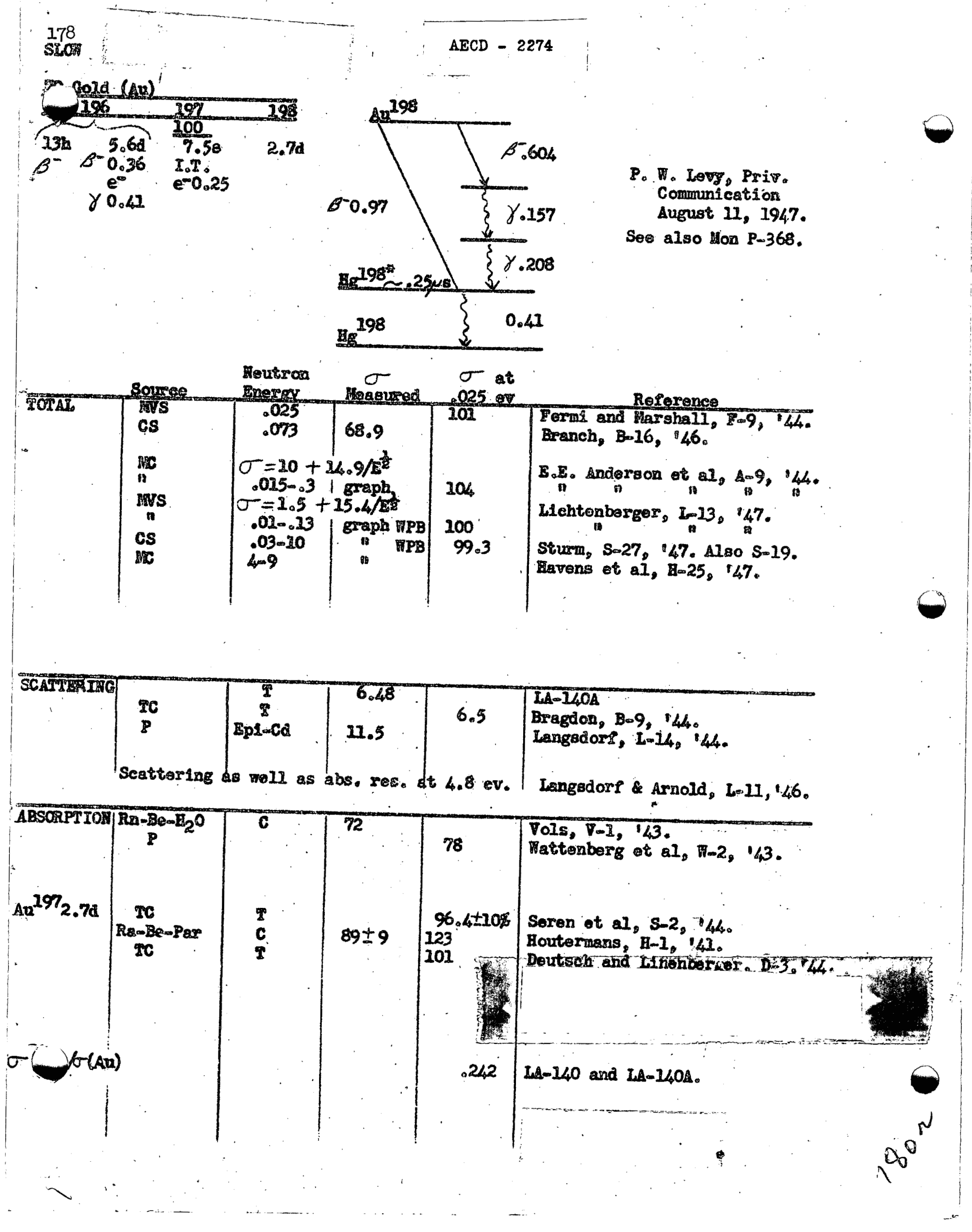


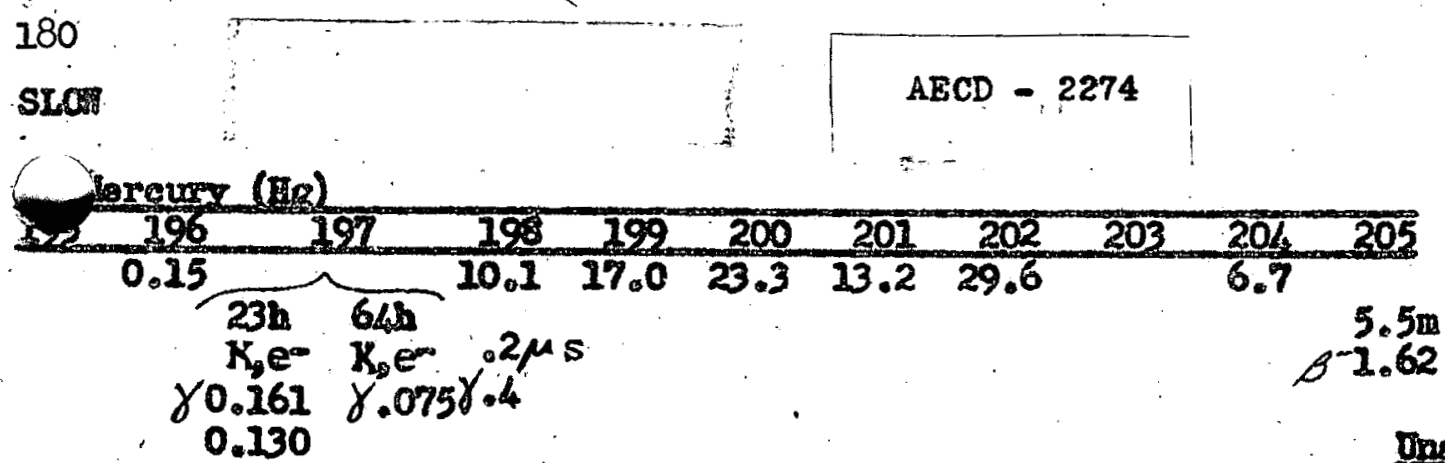

Drassigned Activities $43 \mathrm{~m} \quad 51.5 \mathrm{~d}$

I. $1 . P<0$

$-0.53 \gamma 0.28$

\begin{tabular}{|c|c|c|c|c|c|}
\hline & Soureo & $\begin{array}{l}\text { Meutroa } \\
\text { Engrex }\end{array}$ & $\underset{\text { Heagured }}{\sigma}$ & $\begin{array}{c}\sigma \\
025\end{array}$ & Reforence \\
\hline TOTAL & 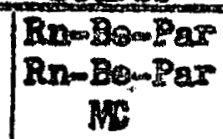 & $\begin{array}{l}\mathbf{c} \\
\mathbf{c}\end{array}$ & $\begin{array}{l}380 \\
445 \\
540\end{array}$ & & 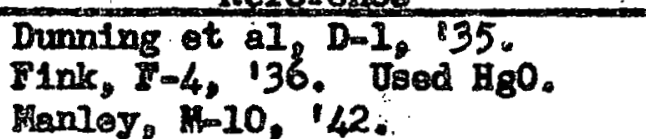 \\
\hline & $\begin{array}{l}\text { CS } \\
\text { cs }\end{array}$ & $\begin{array}{l}.03-.25 \\
.01-.25 \\
.01-75\end{array}$ & $\begin{array}{l}\sigma=67 / \mathrm{s}^{\frac{1}{2}} \\
\text { graph WPB } \\
\text { Congt. tor }\end{array}$ & $\begin{array}{l}600 \\
400\end{array}$ & 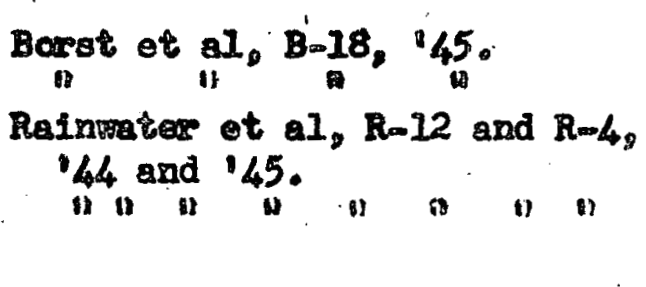 \\
\hline
\end{tabular}

SCATTERIMG $\mid$ Ra-Be-Per

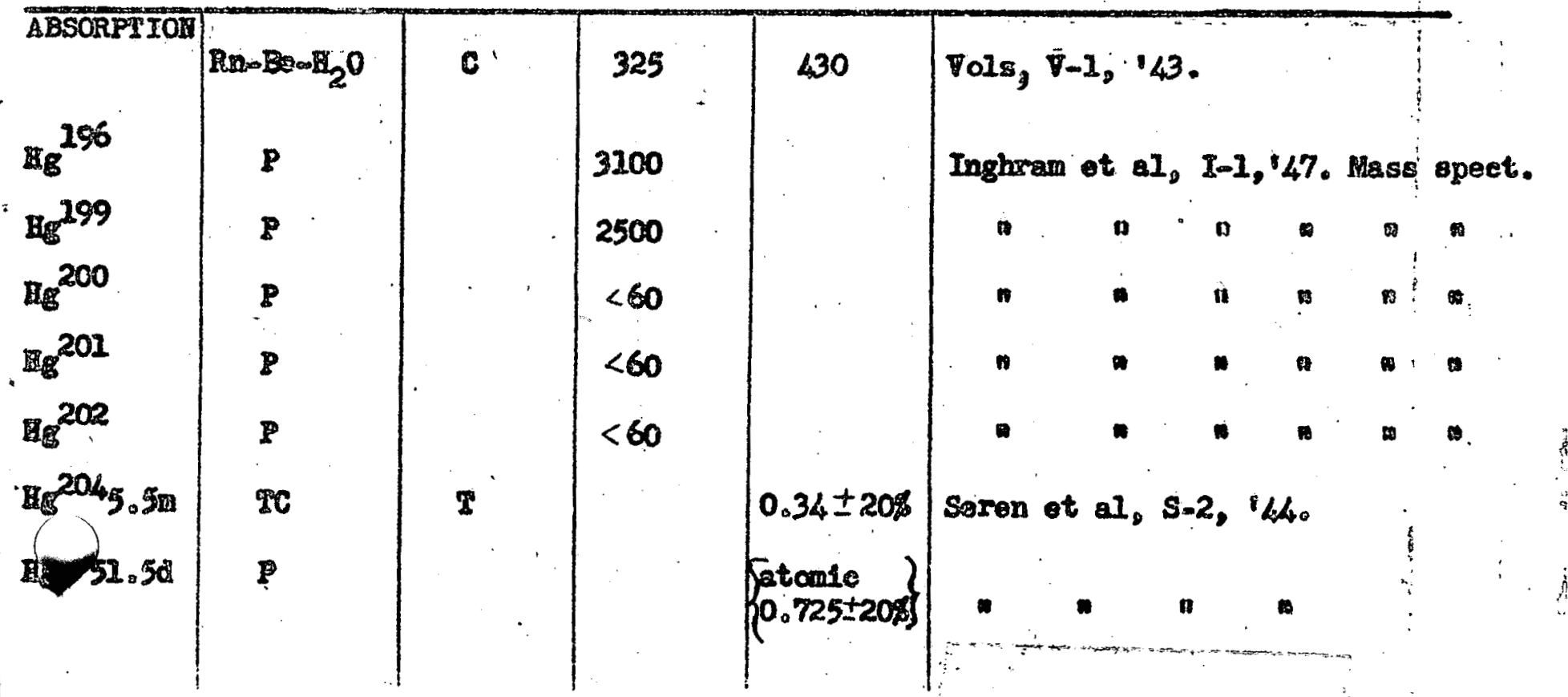




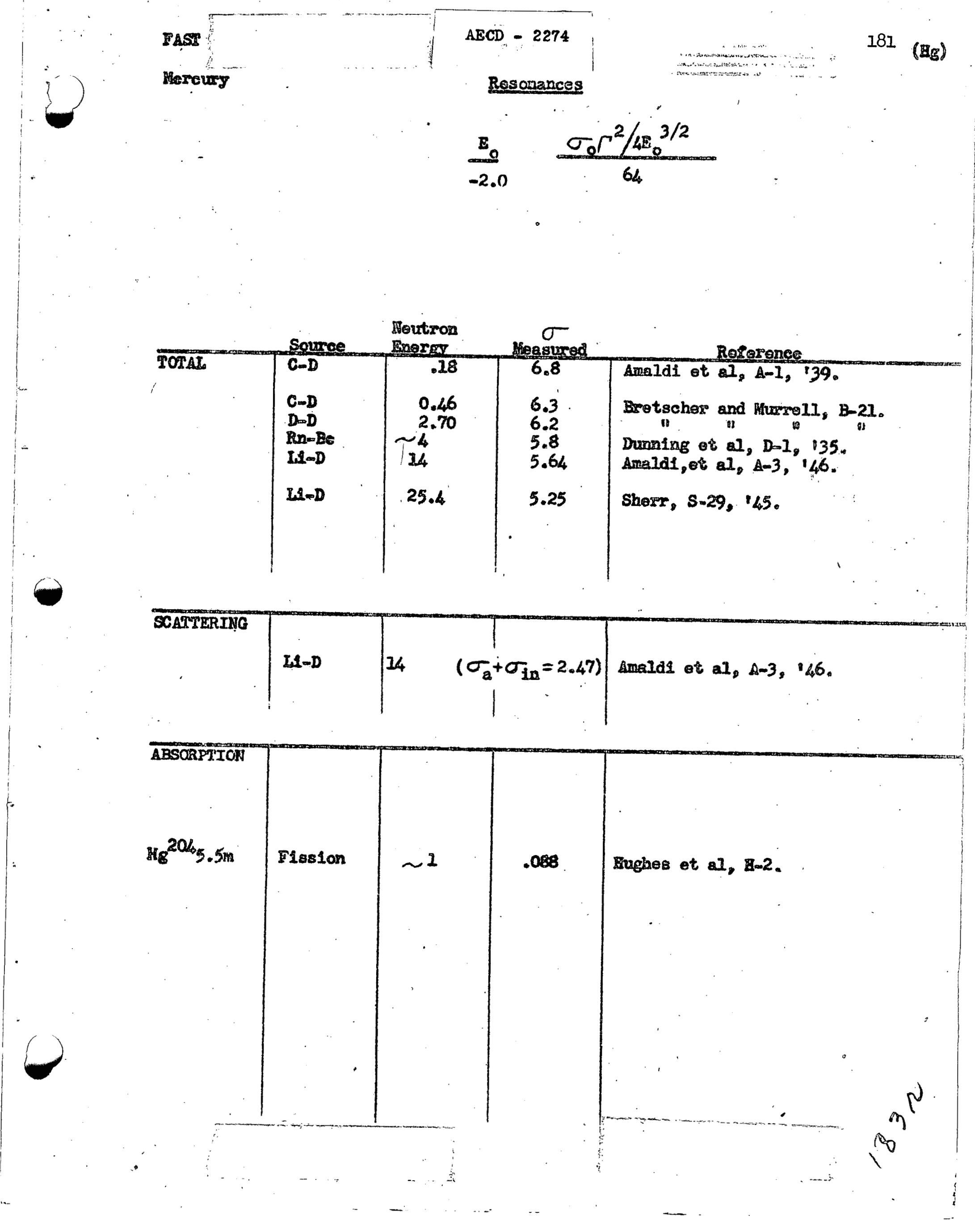




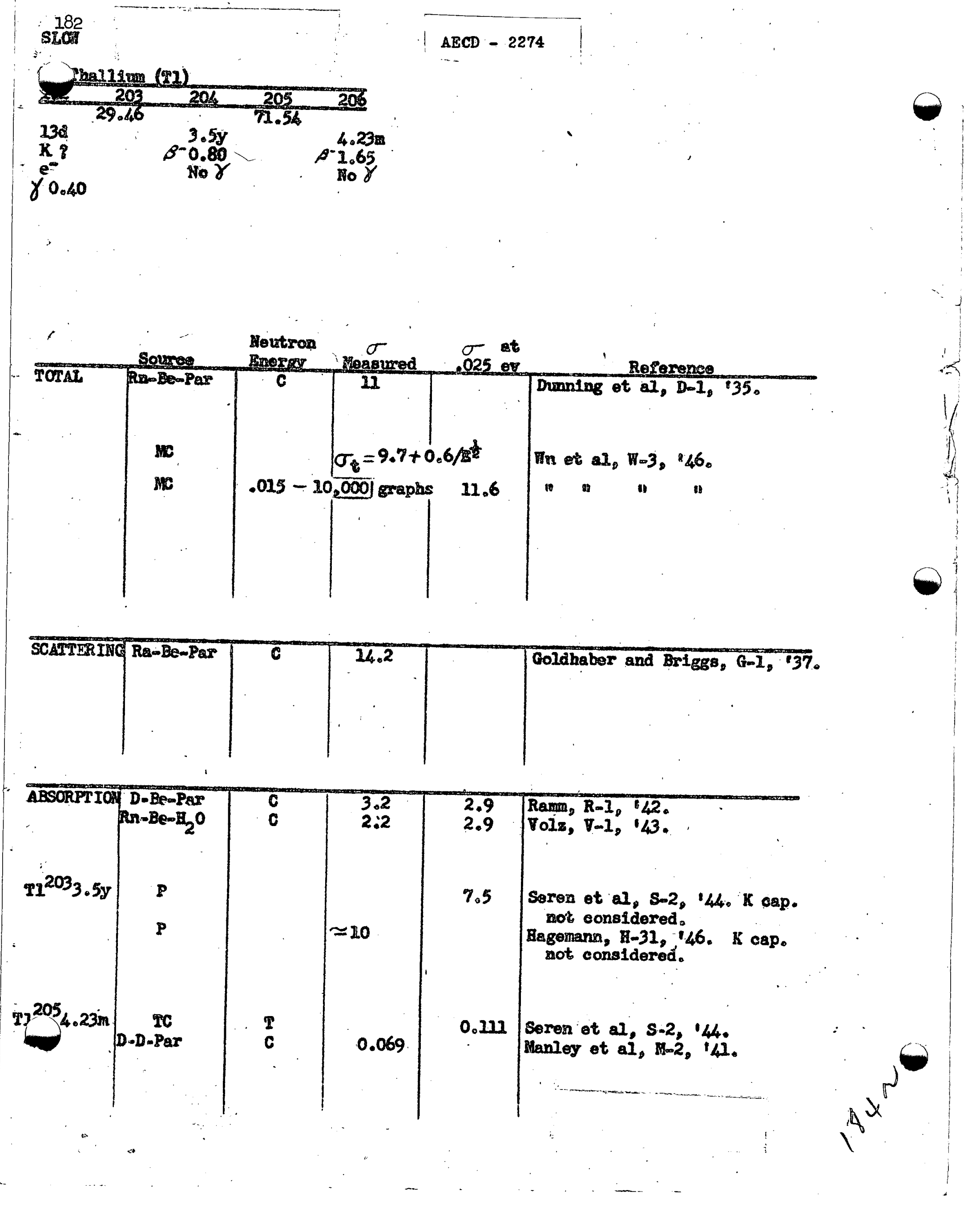




\section{Resonances}

$$
\frac{E_{0}}{270} \frac{\sigma_{0} \Gamma^{2}}{20000}
$$

\begin{tabular}{|c|c|c|c|c|}
\hline & Souree & $\begin{array}{l}\text { Meutron } \\
\text { nerge }\end{array}$ & $\begin{array}{c}G \\
\text { Measured } \\
\end{array}$ & Reforence \\
\hline TOTAL & $\begin{array}{l}\operatorname{Ra}-\gamma=B C \\
\operatorname{Th}=\mathrm{D} \\
\mathrm{C}-\mathrm{D} \\
\operatorname{Th}-\mathrm{B} \\
\mathrm{D}-\mathrm{D}\end{array}$ & $\begin{array}{l}\sim .09 .2 \% \\
0.18 \\
0.46 \\
0.7 \\
3.00\end{array}$ & $\begin{array}{l}11.3 .10 .0 \\
6.5 \\
6.4 \\
6.8 \\
5.2\end{array}$ & 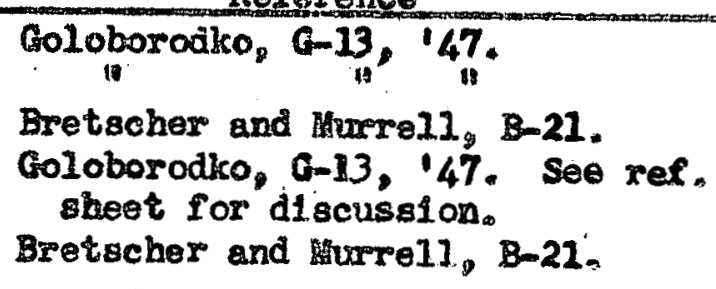 \\
\hline
\end{tabular}

SCATTERING

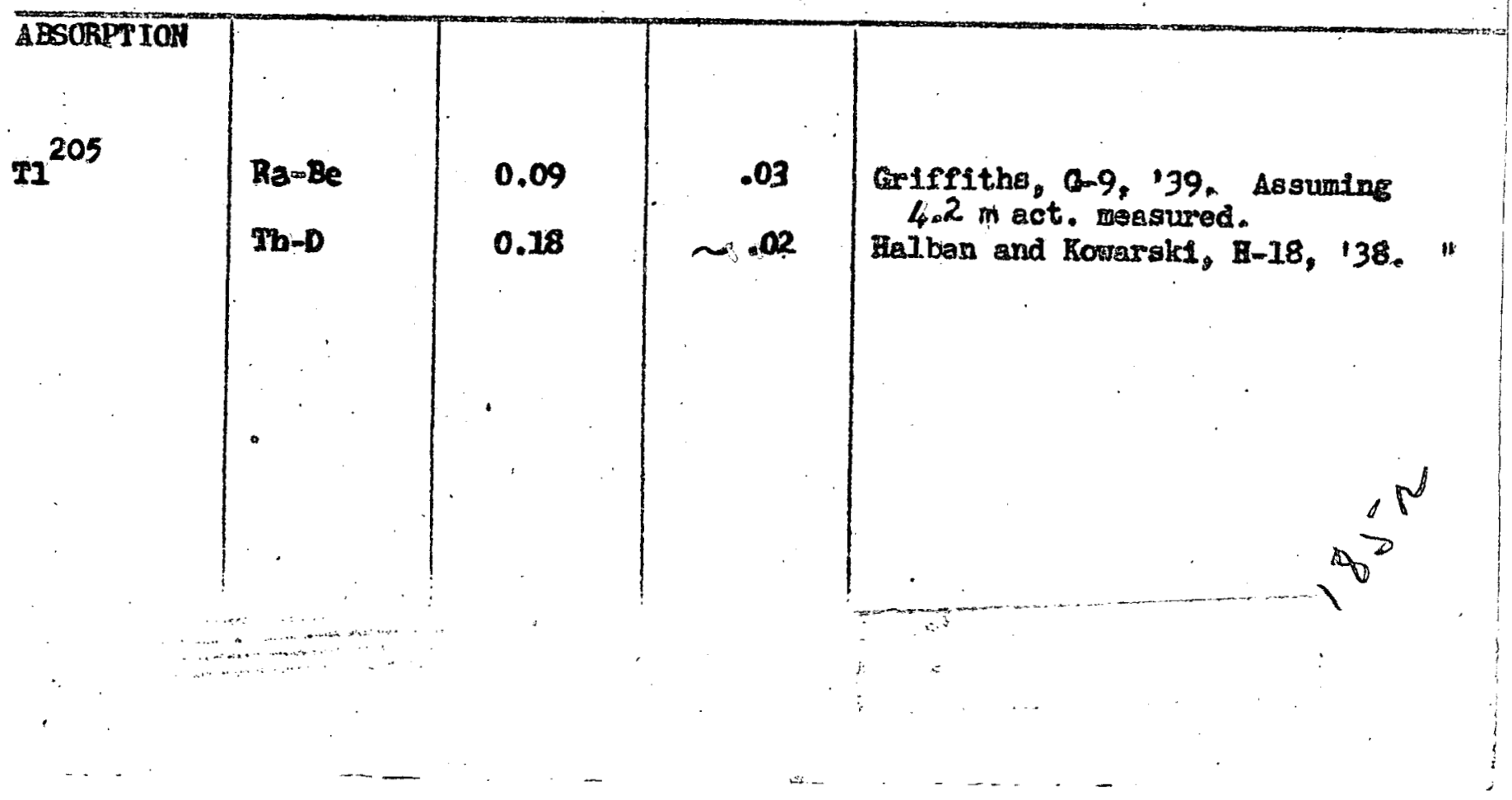


- p lsad (Pb)

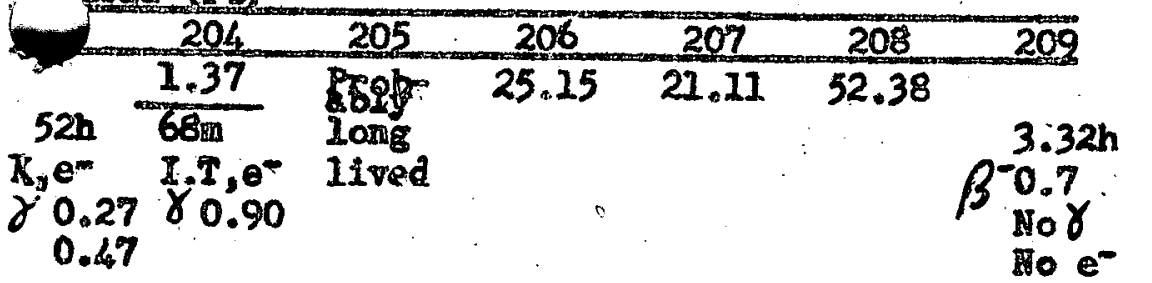

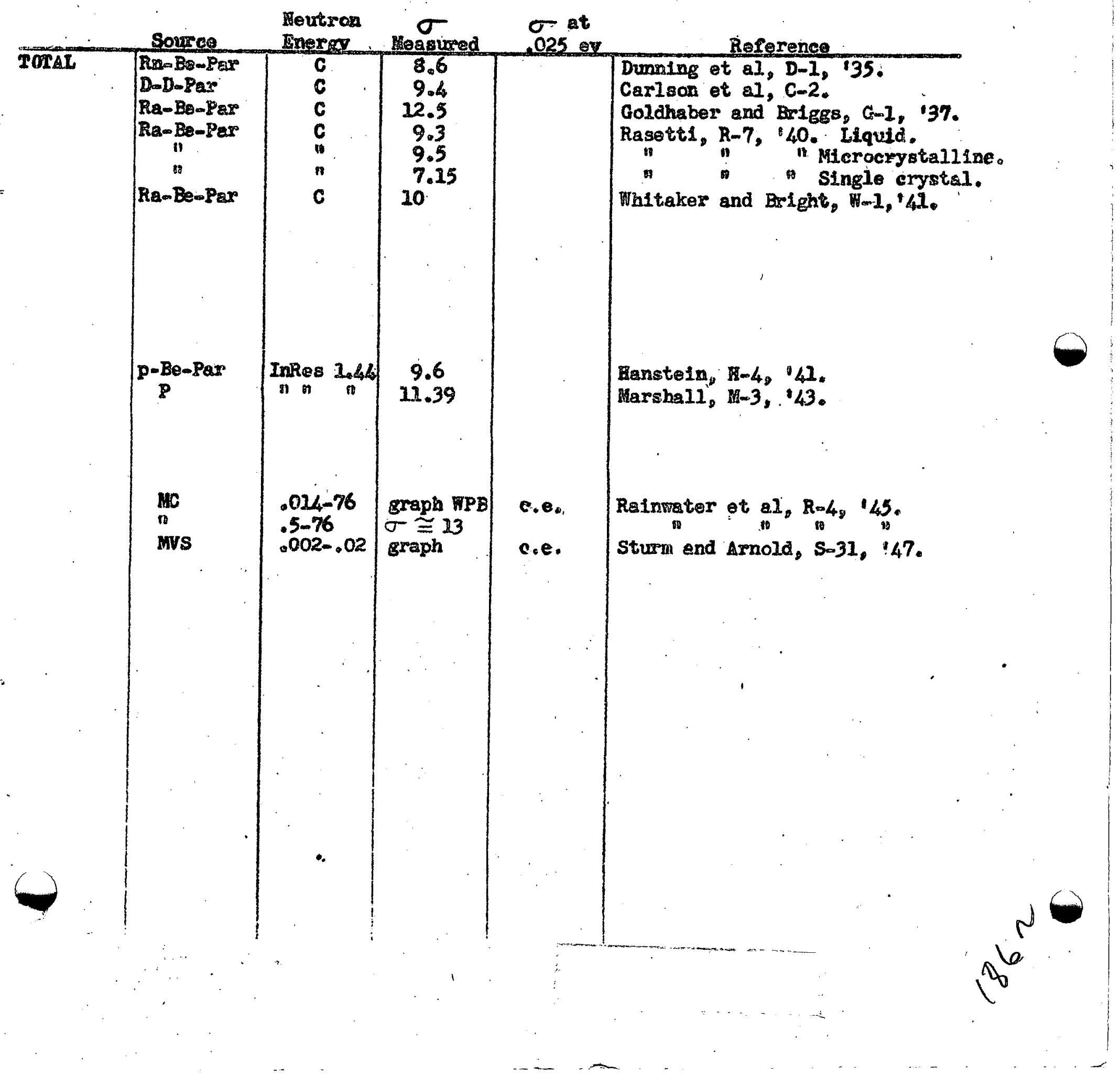




\begin{tabular}{|c|c|c|c|c|c|}
\hline & Soures & $\begin{array}{l}\text { Heutron } \\
\text { Energ }\end{array}$ & $\begin{array}{c}\sigma \\
\text { 盖egsured }\end{array}$ & $\sigma_{025 \mathrm{ev}} \mathrm{at}$ & Reference \\
\hline SCATTERTHA & $\begin{array}{l}\text { TC } \\
R a-B a-P a r \\
R a-B a-P a r \\
R a-B e-P a r \\
R a-B a-P a r \\
R a-B a-P a r \\
R a-B a-P a r \\
R \\
R a-B a-P a r\end{array}$ & $\begin{array}{l}T \\
c \\
c \\
c \\
c \\
\text { RhRes } 1.29 \\
\text { InRes } 3.44 \\
\text { AgRes } 5.1 \\
\text { Epi Cd } \\
\text { I Res } 37\end{array}$ & $\begin{array}{l}10.8 \\
6.2 \\
12.9 \\
12.0 \\
9.0 \\
6.6 \\
10.5 \\
12.3 \\
12.0 \\
13\end{array}$ & & 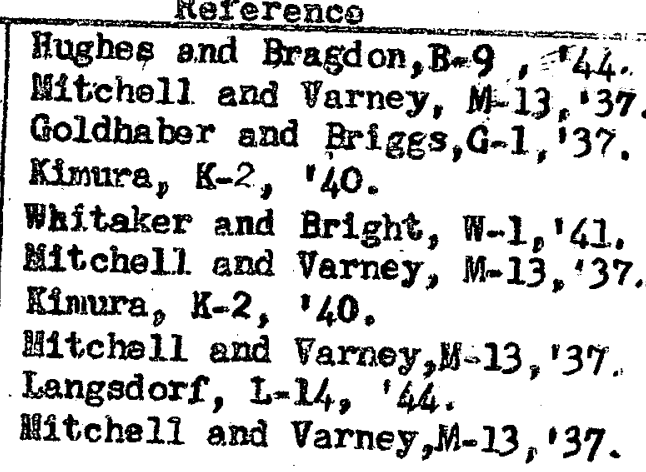 \\
\hline 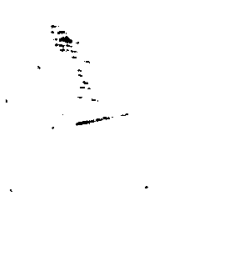 & . & $\underset{\text { Residual }}{4 \pi a^{2}}=$ & $v_{s}^{2.9}=0.85$ & & Fermi and Marshall, $F-3,: 47$. \\
\hline
\end{tabular}

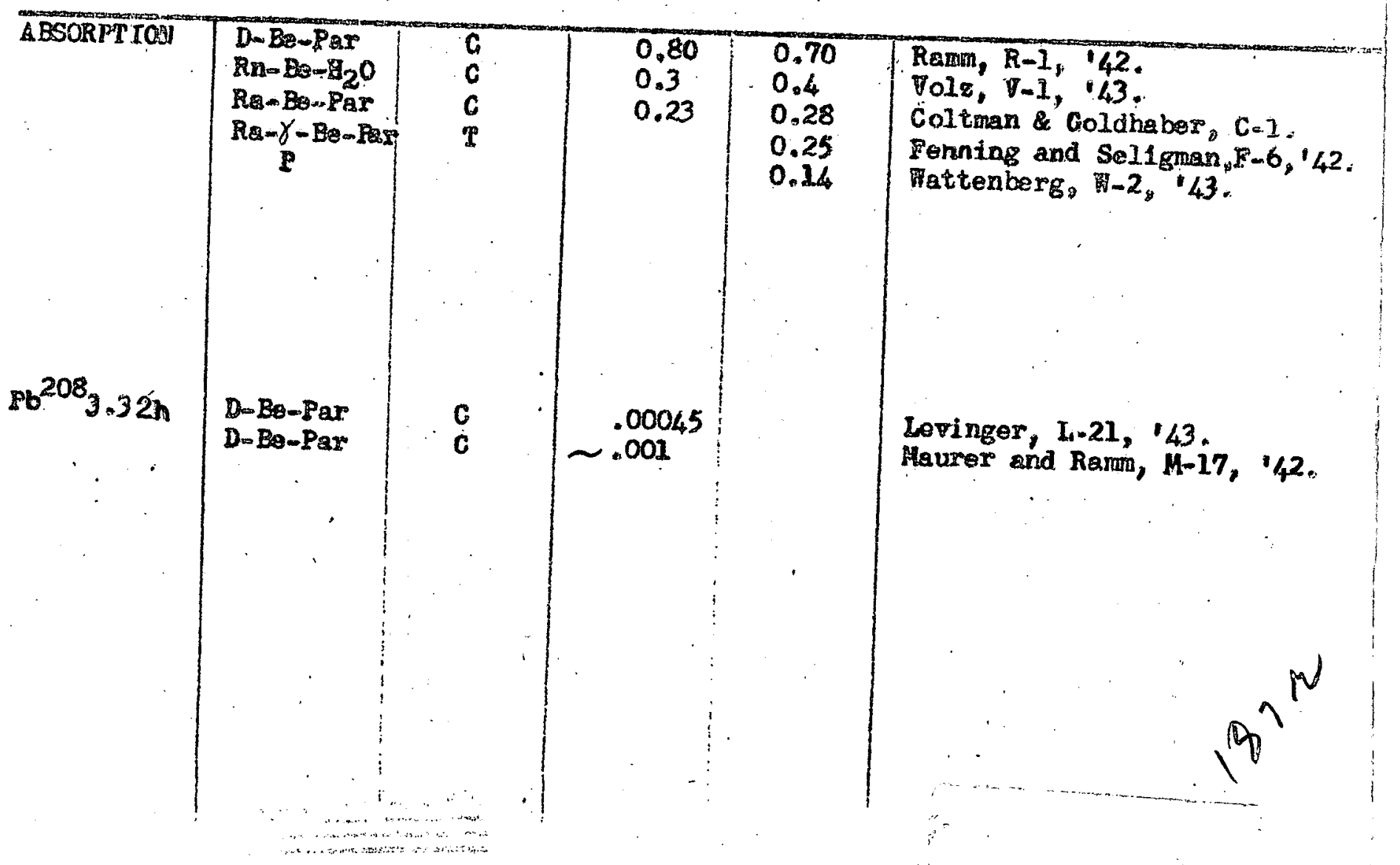


Lead

Resonanese

$\mathbf{E}_{0}$

\subsection{Possible}

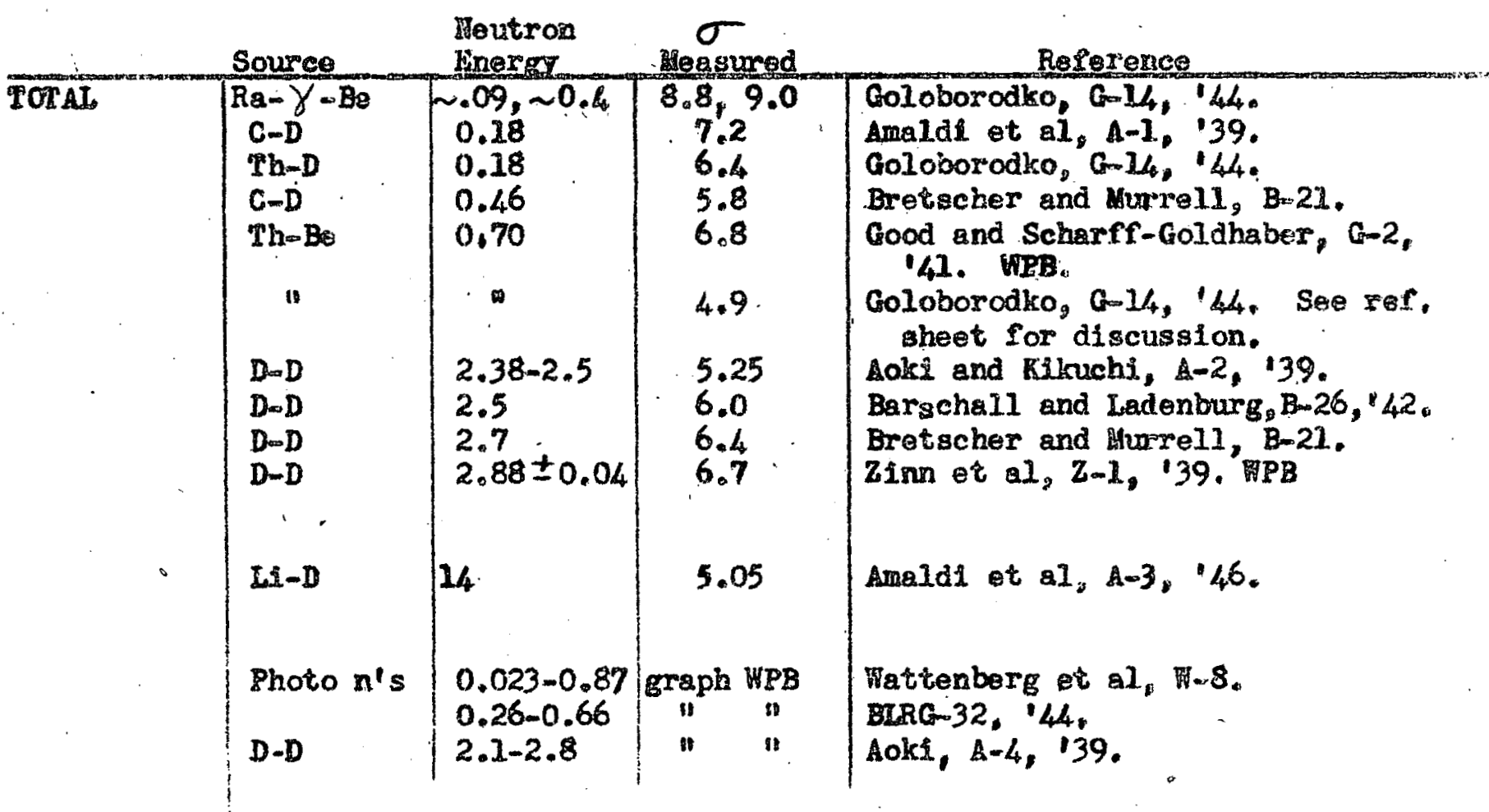

SCATTERMG For inelastic, difforential and transport cross sections,
seo reference sheet.

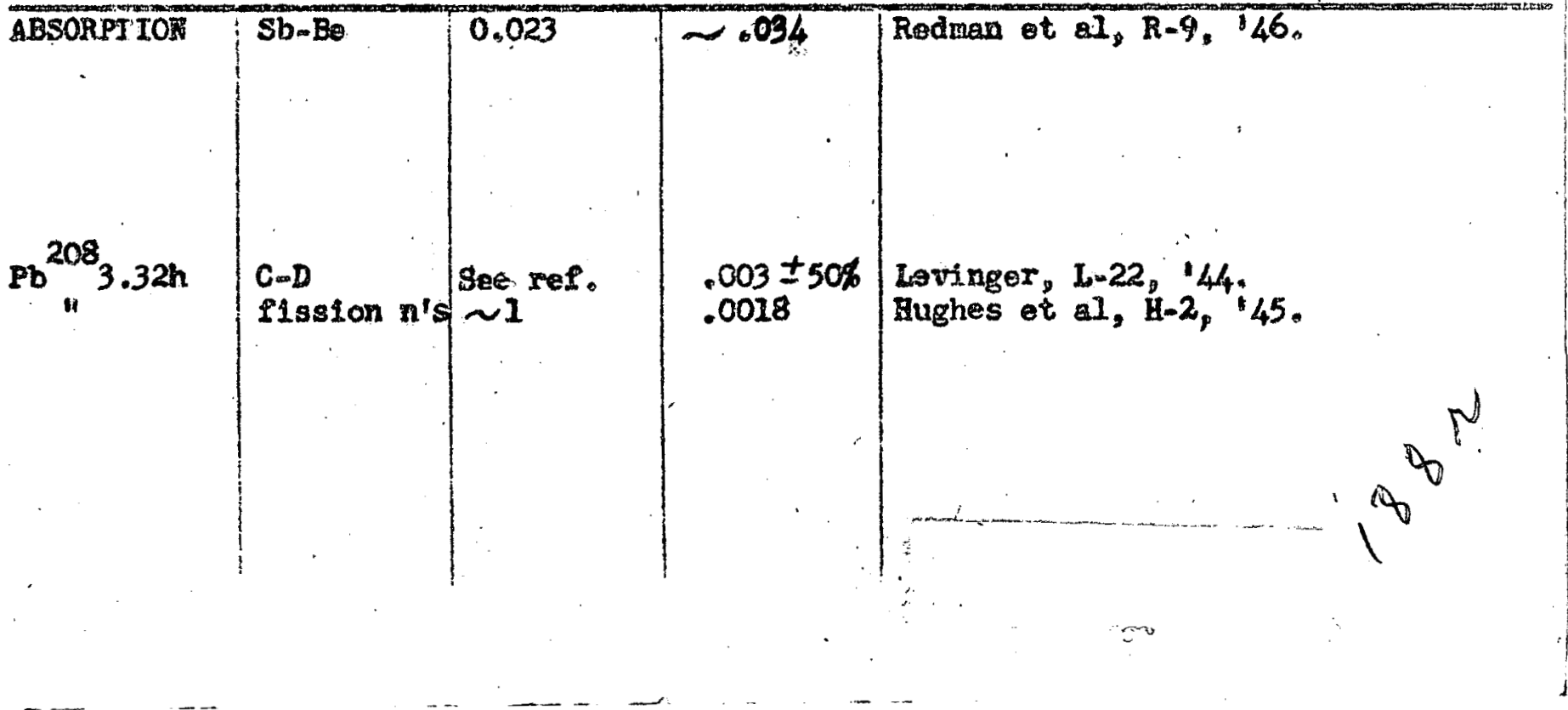


$8^{2}$ 1smuth (BI)

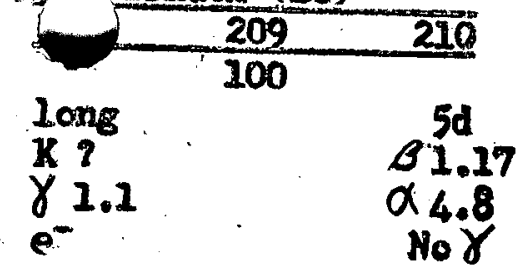

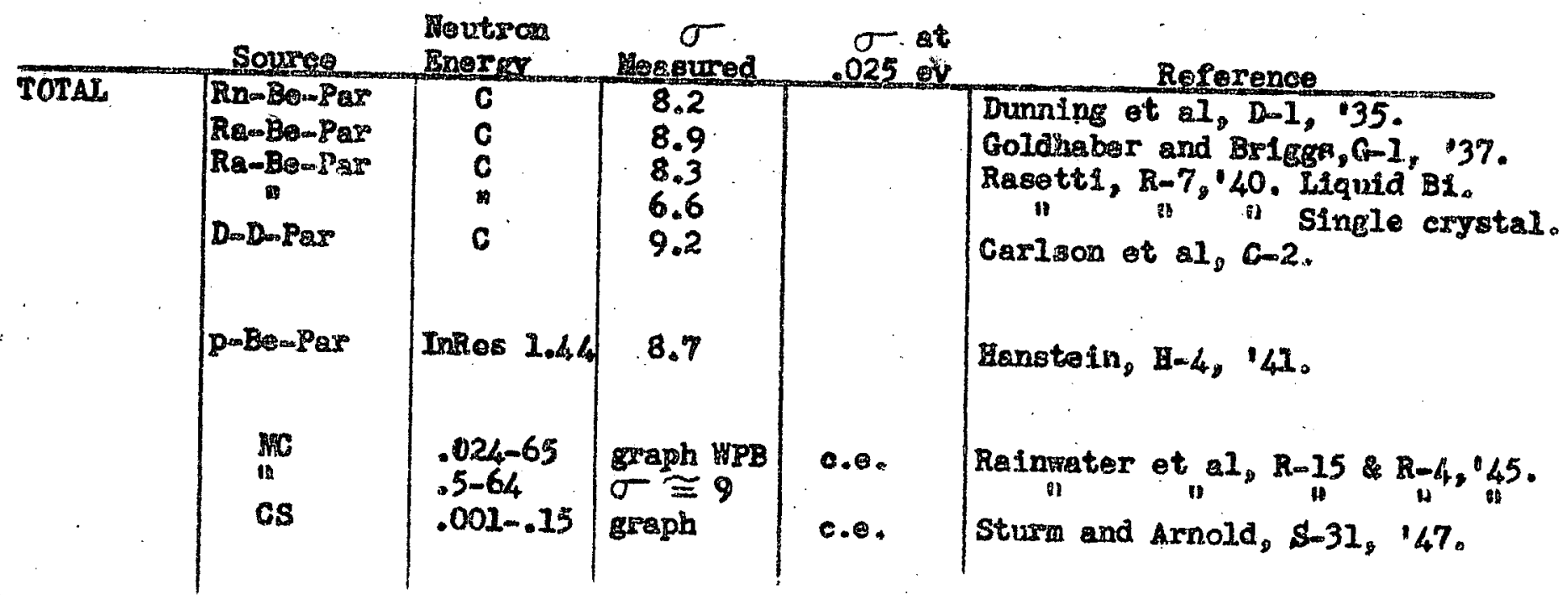

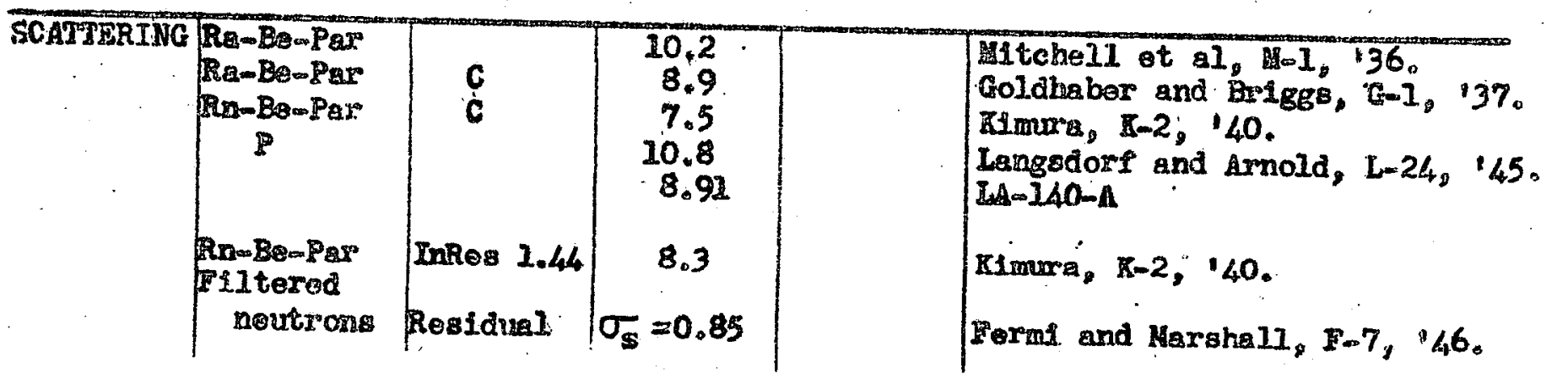

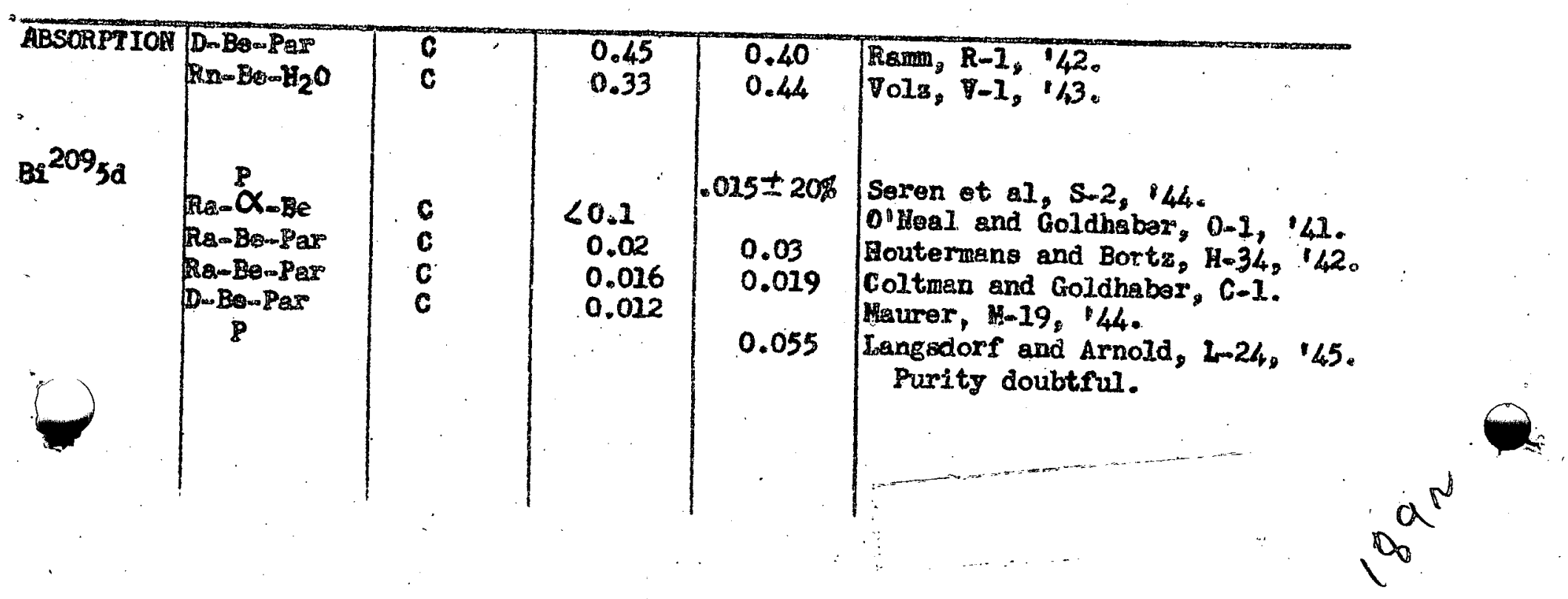




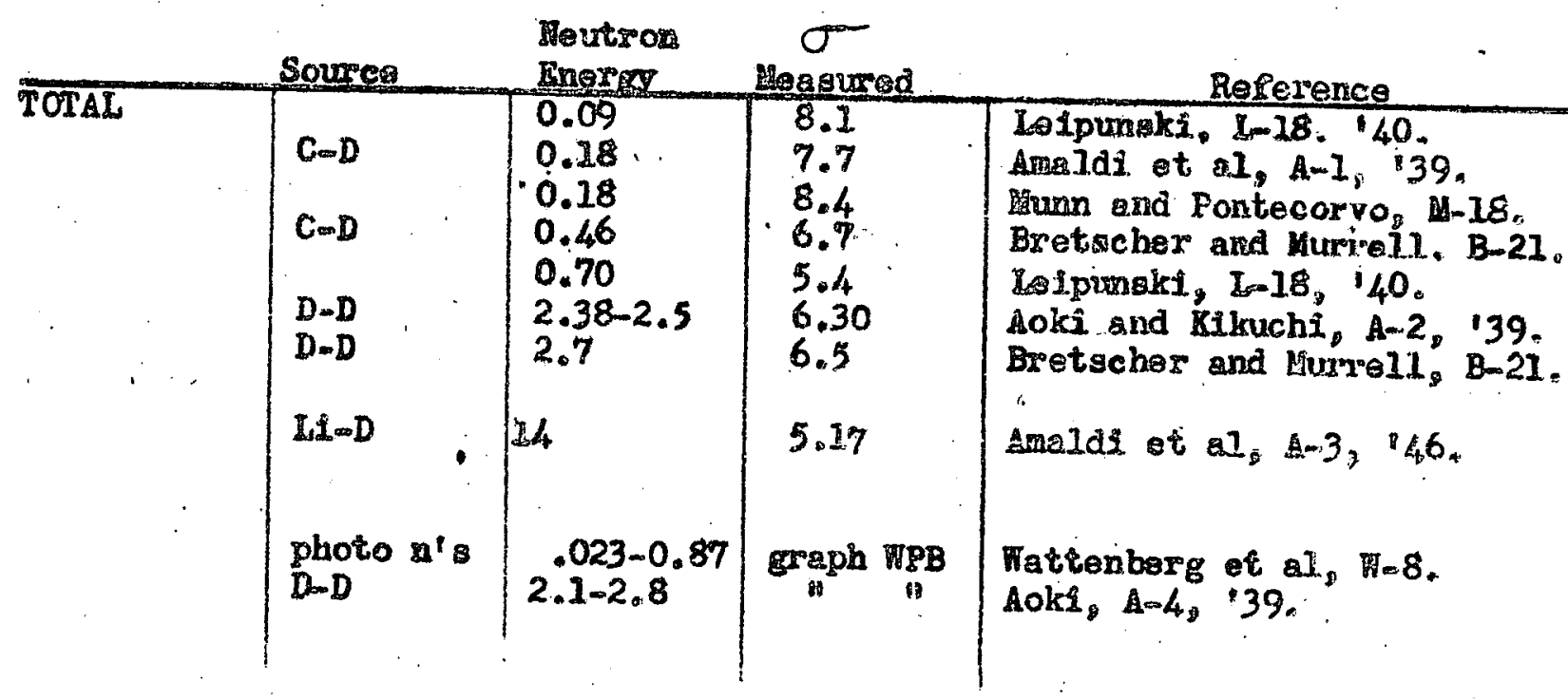

$\left|\begin{array}{c|c|c|}1 \\ \text { SCATTERING referesces to differential and } \\ \text { seeference shoet. }\end{array}\right|$

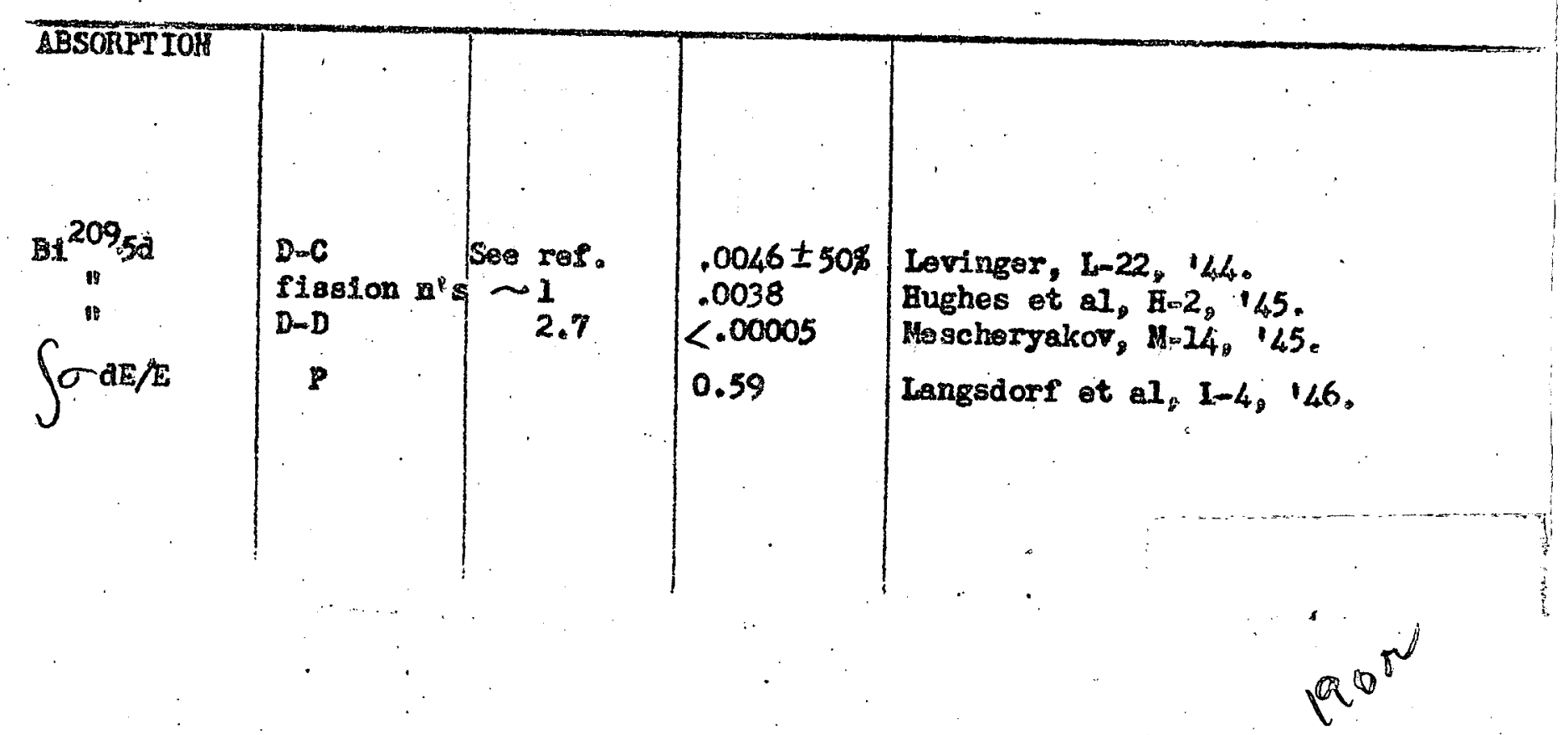




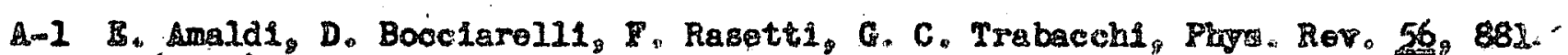
(1939). Heasured total cross sections of 39 eloments by transmission method uBing $C \rightarrow D$ neutrons of estimated effective energy 0.18 mev。 wook $Q=0.28$ mer. ade $5 \%$ correction for inperfect geometry.

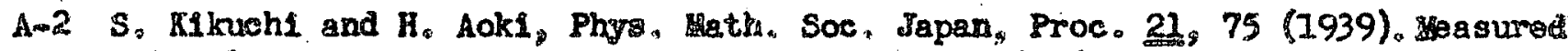
total cross sections by transmission method of 38 elements using $D=D$ neutrong whose energ lay between 2.32 and 2.50 mer. Took $Q=3.18$ mey. Use of $\theta=3.32$ mer. would increase energy $0.14 \mathrm{mev}$. This latter is the palus used in plotting the results of Aokl, $Z 1$ in, and HacPhall in the wall papex book (MUC.MHG-7). Value from mases in the project handbook is 3.25 mer。

Cross sections for LI-D neutrons were also measured but these were not ineluded in the tables because of the wide energ spread of the neutrona.

A-3 A Ameid1, D. Bocciarell1 B. M. Cacciapuoti, G. C. Trabacchi, A, Cimento $3,203(1946)$. Used $\mathrm{Cu}^{63}, 2 \mathrm{n}$ resction which has threshold at il mev to detect neutrons produced by Li-D reaction having maximu energy of 14.5 mev. Av rage neutron energy estimated as in 14 mev.

A-4 Aokl, Phys, llath. Soc. Japan, Proc. 21, 232 (1939). Heasurad total cross sectlons. Neutron onergies between $\sim 2.1$ and $\sim 2,8$ mev were obtained by uaing $D-D$ neutrons emfted at different angles with respect to the incident beal. Used $Q=3.18$ mev. In the wall paper book the results have been plotted using neutron energies recalculated by lacPhail using $Q=3.32$ and making allowances for the widtha of the neutron spectra.

A-5 J. H. Anderson, $\quad$ a 100,1942 .

4-6 I. Lo Anderson, E. Fermi, and L. Moods, CP-781, 2943, and CP-718 (1943).

A-7 J. R。 Arnold and A. Sugarman, CC-3785, 1947 , . J,

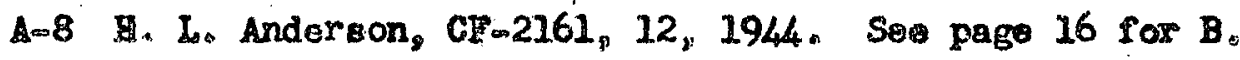

4-9 E. E. Anderson, L. S. Lavate1I1, B. D. Modaniel and R. B. Sutton, LA-93 and 1000-232, 1944.

A-10 Lo Aaderson and E. Fermi, 1-2, 1941.

All Ageno, E. Ameld, D. Bocciarelli, G, C. Trabacchi, Fhys, Rev, 11, 20 (1947). Total eross sections of $B_{3} D_{3}$ and $C$ measured for neutrons with energies of $4.1,12.5$, and 13.5 mer. Pxoton recolls in forward direction detected by means of three proportlonal coincidence counters.

Joural title abbreviations of Lhysles sbstracts uead. 
A-12 I. Io Anderson, B。 Mollog, and D. Hegle, CPa718 (1943)。

A-13 8. Auger, A. H. Hund, B. Fontecorvo, Mp-61 (1944) and Can。 J. Res. 25A, 143 (1947). A-14 I. I. Anderson, CP-155 (19/2).

B-I B. Boake and H. Reddemann, Z. Phys. 220, 56 (1942). Heasured total croso sectlons of a number of rare eartha by transmission measurement using RaTh $\rightarrow B e$ source in paraffin and Dy detector. Cd differences teken and geometry corroctions made. Estimated amounts of impurities in samplos by means of I-ray spectrograph, Host big absorbers present to $<0.18$. Hade corrections for impurities and for water adsorbed during experiment. Value for Cd 1s 2640 which is in good agreesrent with relocity selector measurements.

B-2 F. Go Brlckwedde, Jo R. Dunning, H. J. Hoge and J. H。 Manley, Phys. Rev。 24, 266 (1938): Heasured transmission of 11quid $\mathrm{H}, \mathrm{M}, \mathrm{O}$ and $\mathrm{H}_{2} \mathrm{O}$ for neutrons fros $\mathrm{Rn}-\mathrm{Be}$ source slowed in paraffin kept at room temperature and at liquid air temperature.

B-3 L. B. Borgt, A. J. Urich, C. L. Osborne and B. Basbrouck, Phgs. Rev. 70, 557 (1946) and CP-2601. Neutron diffraction studies with crystal spectrometer.

Buld E. Bleuler, W. Boltmann and W. Zunt1, Helv. Phys. Acts 19, 419 (1946).

B-5 H. E. Bradbury, F, Block, H。Totel and Po A: Ross, Pays, Rev。 22, 1023 (1937).

B.6 H. Go Beyer and $u_{0}^{\circ}$ D. Whitaker, Phys, Rev。 57, 976 (1940)。 Rn-Be source is paraffin. Cd difference takem.

B.7 E, Bleuler, W. Zunt1, Helv. Phys. Acta 20, 195 (1947).

B-8 H. Bradt, P. C. Gugelot, 0 . Huber, H. Medicus, P. Prelswerk, P. Scherrer, R. Steffon and E. T. H. Zurich, Helv. Phys. Acta 19, $219 \cdot(1946)$.

B-9 E. Bragdon, V.C. Wilson, D. J. Hughes, CP-1827, 10, CP-2081, 28 and CP-2305, (1944). Scattering cross sections rere measured in a back seattering geometry in the thermal columa. Heasurements pere relative to graphite whose value pas taken to be 4.8 . CP -2305 gives descript10n of method and sumary of results.

B-10 I. Bradt, P. C. Gugelot, 0. Huber, H. Medicus, P. Preiswerk, P. Scherrer, R. Steffen and E. T. H. Zurlch, Helv. Phos, Acta 19, 221 (1946). 


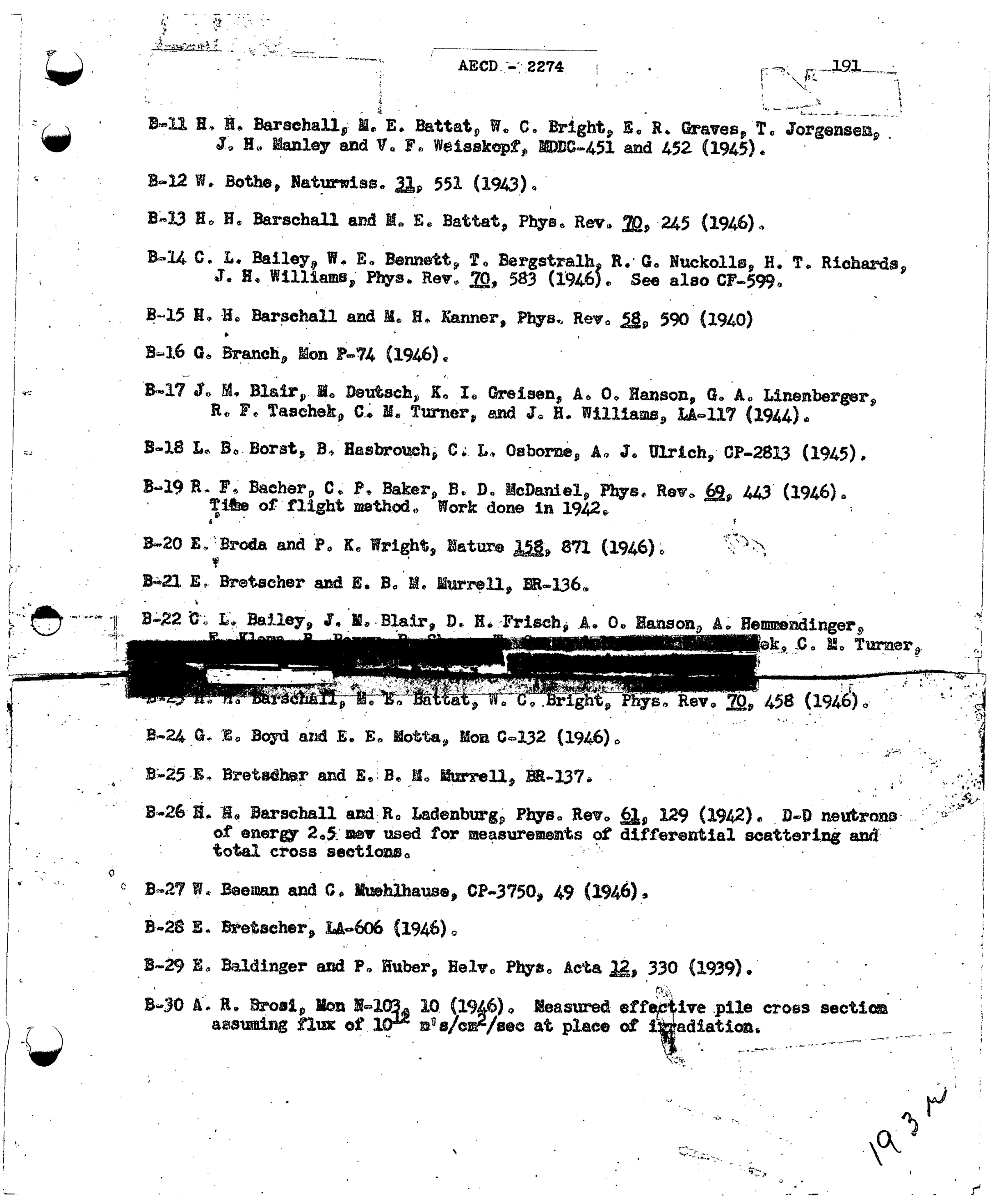


E-31 D. Bobm and C. Richmen, Phgs, Rov。23, 567 (1947)。

Z32 R. F. Bacher, C. P. Baker, M. G. Holloway and B. D. HeDaniol, CP-1446 (I942)。

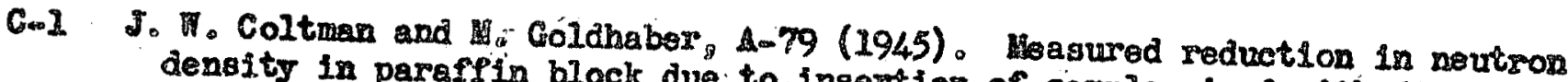
density in paraffin block due to ineertion of ample.mixed with inely divided carbon. Placed 100 Ra-Be source near botton of block and used $\mathrm{BF}_{3}$ chamber. Apparatus calibratod by mixing finely divided BC Fith $C_{0}$ Heasured $\sigma^{-1}$ based on $\sigma(B)=600$. Values for $\sigma$ at .025 or 年 $730 / 600=1.217$

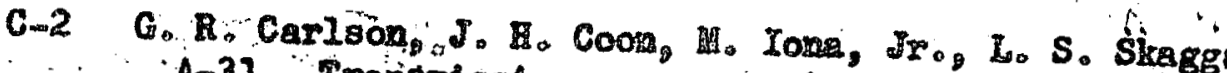
A-31. Tranismission peesurements and detseted with $\mathrm{BS}_{3}$ counter.

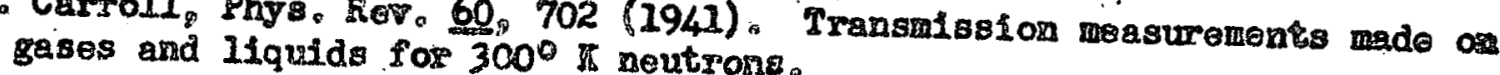

G. I. Ho Coon, CP-107 (1942). Lethod sinilar to that of Coltran and Goldbabar.

Cos 5 W. E. Cohn and group, nor S-29 (1945)。

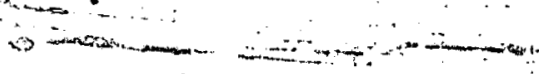

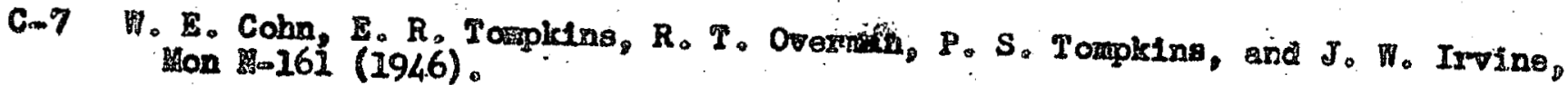

C.8 8 . Camse and L. Brom, CP-2407 (1944)。

C-9 B. Chudon and C. Oo llovinhause, CP-3801, 25 (1947).

C-10 V. P. Cohen, H. H. Coldsw1th, and J. Schwinger, Phys, Rev. 25; 106 (1939).
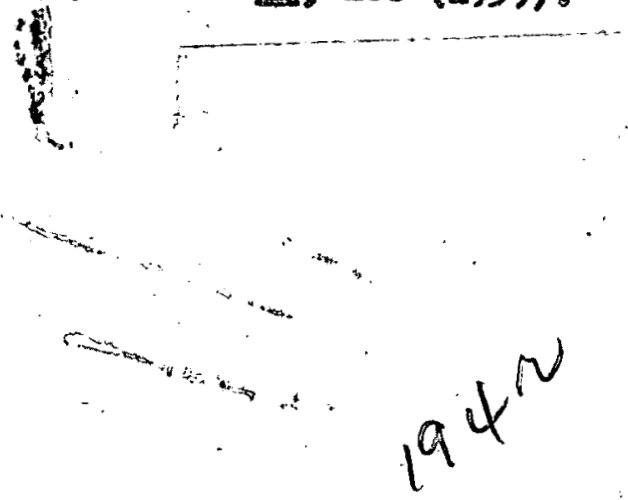
E-1 C. Bgeler and C. Huddleston, GP-364\%, 12 (1946).

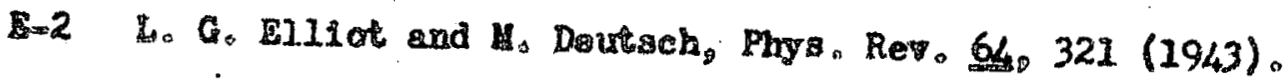

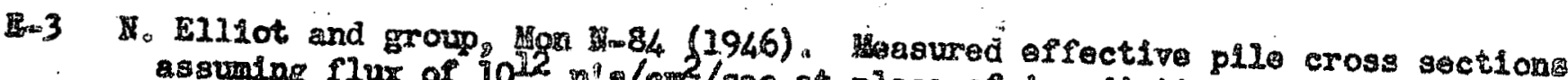

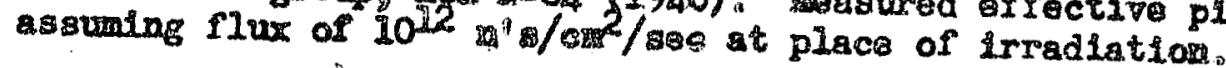

$E=4 C_{4}$ Eggler and Jo Fallace, Cra 3574,31 (1946). Dangepr coefficient mothod.

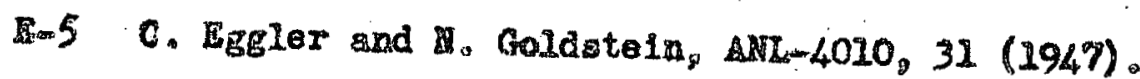

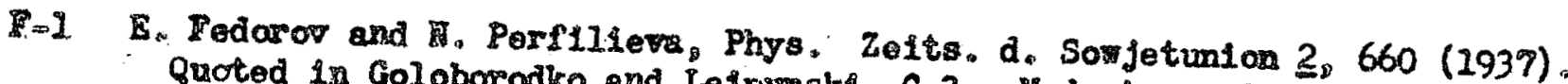

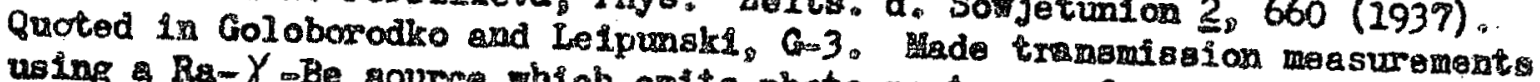
using Ra- $\gamma$ mbe ource which emits photo neutrons of $\sim 90$ and 400 kev

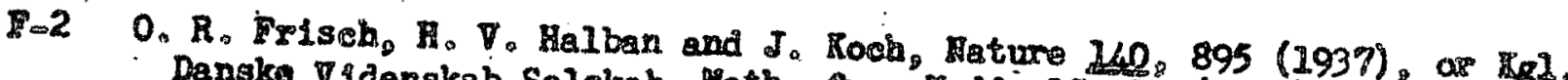
Danske VIdenskab Selskab, Hath. Tys, cross seotions fron nember of poutrons foud in colutions around Rem

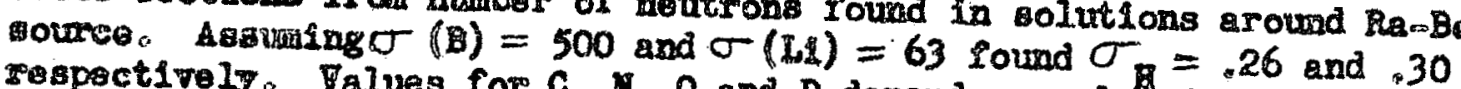
respectively, Values for $C_{2}, O$ and $D$ depend on valu for, 26 and 30 $2200 \mathrm{w} / \mathrm{s}$. Total aross on $\sigma_{H}=.33$ and are for a neartras Folocity of wothod for parafin slored noutron also measured by the transmission energy not clear but found $\sigma(A n)=0$ Cd differences wero taken. Neutron $\sim 4025$ ช\%.

P-3. Ferml and $L_{0}$ Larshall, Phys, Rev, Ito 666 (19/4). Scattoring lengths mere calculated from crystal interference and total reflection effects In the iables the scattering cross sections for pres atom as calculated $(7946), \mathrm{CF}-3574,38(1946)$ and $\mathrm{CP}$ iven as $4 \pi \mathrm{a}^{2}$, See also CF 003490,33

F-4 G. A. Fink, Phys, Rev. 50, 738 (1936). 


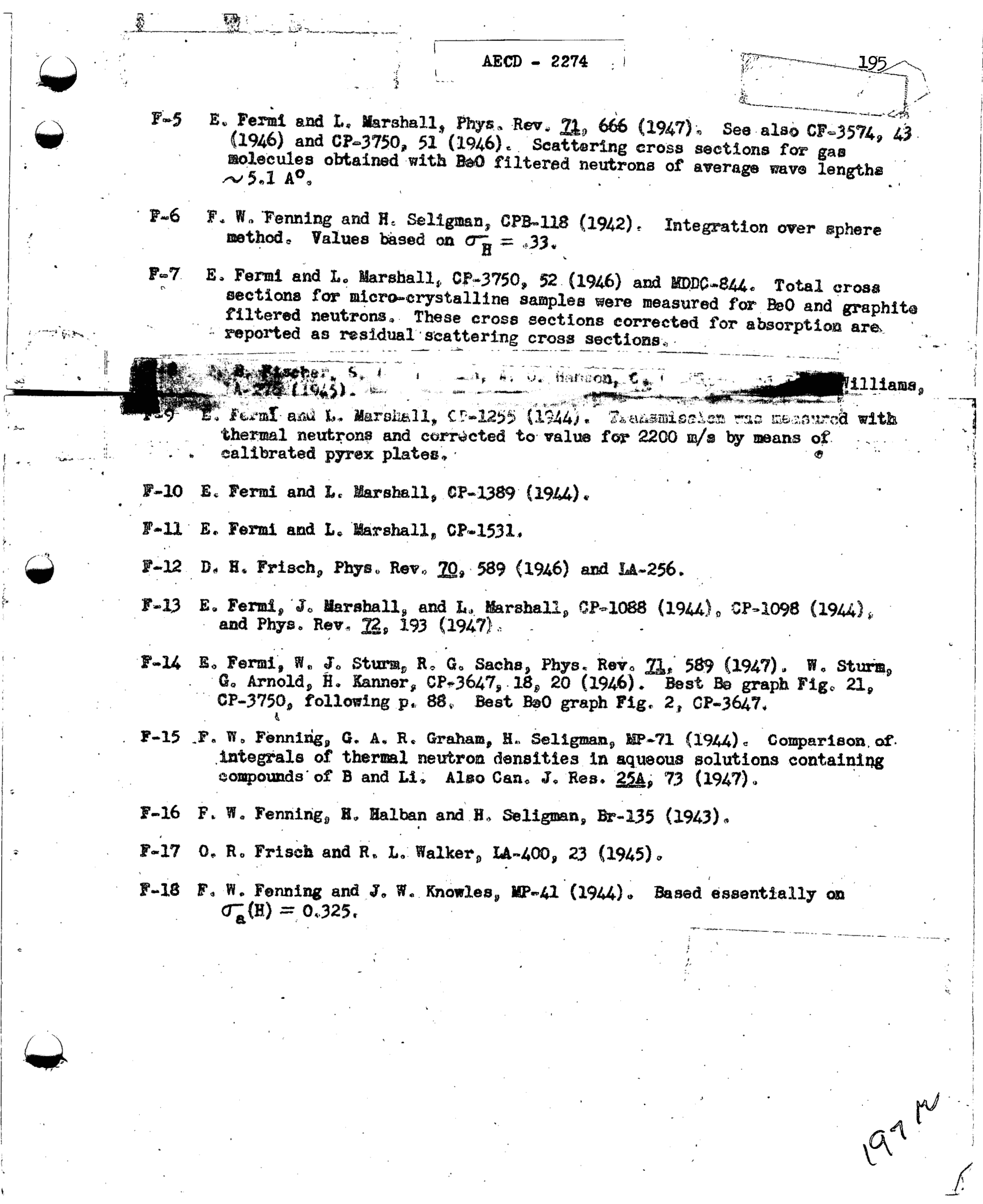


Q-1 H. Goldhaber and G. A, Arlggs, Proc Rog, Soc. 162,127 (1937). Trensmission measurements. Less scittering into detector than in Dunaing arrangement which tends to make results lower. No correction for limperfect geometry. Scatterling cross sections. for angles of $\sim 90^{\circ}$. also messured for $C$ neutrons. relative to $C_{4}$ fotal ecattering cross eecticas ae 10.

G.2 W. E: Good and G. Scharff-Coldhaber, Phys. Rev. 59, 917 (1941).

Go3 I. Goloborodko and A. Leipunsk1, Phys, Rev。 56, 891 (1939). Transmisaios... messurementa, Dy detector imbedded in parasfin used.

G-4 E, E. Graves and J, Manley, GP-1469 (1944).

G-5. Goldstein, W. Spatz, E. Goldfarb, H. Murdock, J. Wallace, D. J. Hughies and C. Eggler, $\mathrm{CP}-3574,28,32,33(1946), \mathrm{np}$ and $\mathrm{a} \alpha$ cross sections mossured for neutrons produced in 0 plates placed in thermel beam or rabbit and in 0 lusisp placed in CB 2. 2rethod and preliminary values given in GF 3490 .

Gur6 T. Goloboyodko, J. Phys., USSR. 5, 15 (1941).

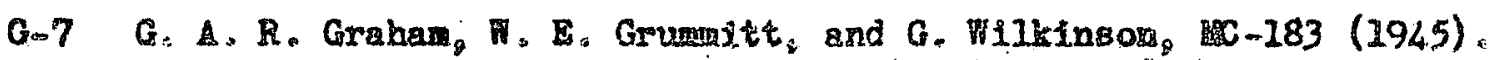

Goo E. Grumitt, J. Gueron and G. Hilkinson, $=70 .(1944)$.

G-9 Jo R. Griffiths, Proc Ró. Soc. 170, 513 (1939). Activation crosa sections for Ra- $\gamma$-Be deutrons. Such a source emits neutrons of several different energies in unknown relative proportions, The in 1.76 met $\gamma$-ray would produee neutrons of energy 0.12 mer in a thin source or 0.09 mev if an energy degradation occurs. Neutrons with energies of 0.4 and higher are also present. (Soe discussion under 6.73 ,) The value 0.09 was chosen rather arbitrarlly for the purposes of these tables. Griffiths' measured value for Au was based on an estimated value for the strength of this source. It was found to colncide with that of Blair et al at the energ 0.09 mev (B-17, corrected for $L A-520$ ) 11 multiplied by a factor of 4.9. All Griffiths' values reported here were multiplied by this quantity. The results found in this way do not have a vary precise measuring but are included because they give the order of nagnitude of activation crose eection in the humdred kilovolt reglon for some elements for which there are no other measurements. 
1-1 F. Go Houtermans, Z. Phys. 118, 424 (1941). Activation cross sections for C neutrons measured with $\mathrm{Ra}$-Be-Par block as source. $\sigma(\mathrm{M})=9.4$ takes as standard. Corrections made for geometry and foll absorption. Value for .025 ov. found by multiplying values given by $13 / 9.4$.

H-2 $D_{0} J_{0}$ Hughas, $W_{0}$ Spats and $N$. Goldstein. Hethod, given in CP 3403 , mas to activate Cd covered foll of material under invegtigation with fission neutrons from 0 plate placed in thermal besm. Identical bare foll activated simultaneousiy. Fast cross section obtained frow knom themal cross section and calculated ratio of fast to slow flux at plate. Effective neutron energ is $\sim 2$ mev, Values given in $10 D C-27, C P=3403$, $C P-3647, C P-3574$ and $C P-3750$. Pinal graph in this last report, Rew measured thermal $\sigma^{i} \mathrm{~g}$ when knom values gave results off curvo。

H-3 D. Hughes, No Spats, and N. Goldste1n, CP-3647, 9 (1946).

H-4 H. B. Hanstein, Phys. Rev, 59, 489 (1941). Neutrons from proton-beryllium reaction in the eyelotron slowed down by paraffin. Transmission for resonance neutrons obtained by means of Cd shield and removable filters and detectors both of In or $I$. Thermal measurements ade with thick I In shield, Gd ffiter and thick In detector.

Ho6 W. J. Horvath and E: O. Salant, Phys. Rev。 59, 154 (194I)。 Absorption measurements made with detector inmediateiy behind absorber. In order to find values at. 025 ev measured values were multiplled by a factor which makes in value 13.0 , namely 1.16 .

H. 7 D. Hughes, CP-2984 (1945) =

H=8 D. Hughes, W. Spatz, N. Goldstoin, E, Goldfarb and C. Huddleston, CP-3750, $28(1947)$. 


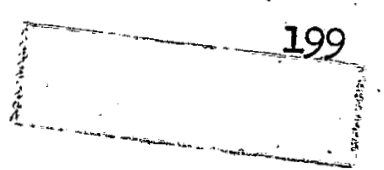

Hos E。 Rineks, Phys, Rev。 20,770 (1946).

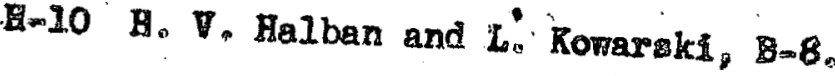

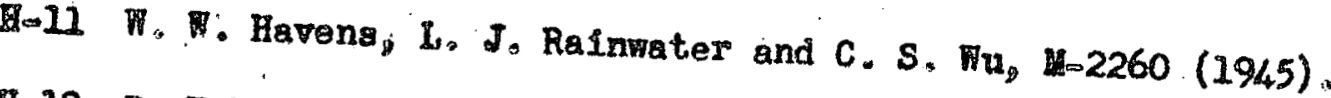

19-12 P. Euber, Hel7. Phys, Acta 24, 163 (1941).

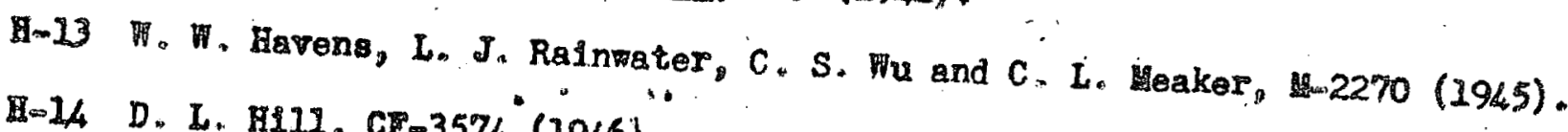

IH -14 D. L. H111, CF -3574 (1946)

H-15 O, Hirzel and H. Weffler, Helv" Phys. Acta 29, 217 (1946)

H-16 W. W. Havens; L.J. Rainwater and C. S, Wu, 2226 (1945).

Hol 17 D. Hughes, E, Bragdon and C, Eggler, CP-1532 (1944).

H-18 H. Halban and I. Kowarski, Matur 142, 392 (1938) cross actions for Tha photo neutrons. by a factor of 17 to bring value por Roported values were multiplied Blair et al (B-17) corrected for IA-520 into greement with that of

A. D. Hanson, E。 D. Rlema, Io Spock and R. F, Taschek, LA-515 (1945).

H-20 0. Huber, R. Huber and P. Scherrer, Helv. Phys. Acta 13, 212 (1940).

Aa-21 D. Hughes, H. Murdock, $N$. Goldstein and E. Goldfarb, CP-3574, 32, (1946).

H-22 D. Hughes, J. Wallece, E. Goldfarb and C. Eggler, CP-3647, 12 (1946).

H-23 W. W. Havens, Jr, and L. J. Ralnwater, AM-2222 (1945)

H-24 E, Jo Horgland and $N_{*}$ Sugarman, CC-2891 (1945).

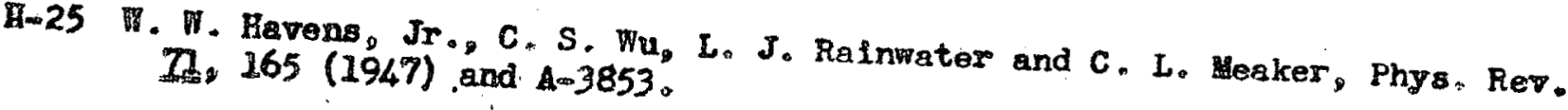

He-26 W. Wo Havens, Jr, and $L_{0} J_{0}$ Rainwater, Phys, Rov, 70, 154 (1946).

y-27 D. J. Hughes and E. Goldfarb, CP-3801, 19 (1947).

H-28 E. L. Harrington and J, I, Stewart, Cangd. J. Ree', 19, 33 (1941).

H-29 D. J. Hughes, $H$, Goldstein and C. Eggler, CP-3795, 19 (1945)

A-30 S. Harr1s, CP-3801, 10 (2947).

H-31 F. Hagemann, CC $-3699,5$ (1946).

\&-32 W. W. Havens and $L_{0}$ J. Rainwater; $4 u_{02} 219$ (1945).

H-33 J. Hinton and I. D. PoKagg, LA-160. (1944).

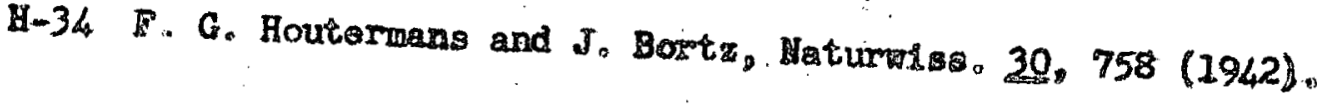




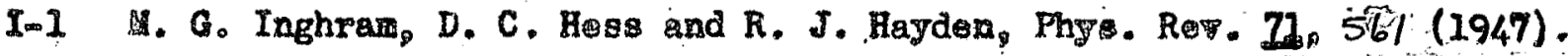

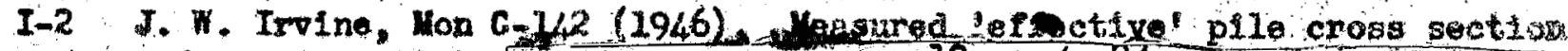
for $\mathrm{ng}$ reaction, dAssumed flux to be $10^{12} \mathrm{n}^{\prime} \mathrm{s} / \mathrm{cm}^{2} / 8 \theta \mathrm{c}$

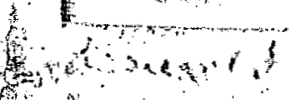

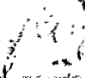

Jo1. H. P. Jesse, CP-1087 (1943)

K-1. S. Katcoff and N. Sugarman, CC-2739, 4, (1945).

K-2 Mo Kimura, Proc. Phys, - Math. Soc., Japan 22, 391 (1940). Beasured back acattering of $\mathrm{C}$ neutrons and indiu resonane noutrons. Compared estimated abolute values with those of Goldhaber and Brigge and concluded no correction factor, necessary.

Hin-3 B. D. Rerm, CP-772 (1943).

X-5 H. Kubitsehals and H. I. Andarsora, CP-1825 (1944). Also CP-182i.

Hik-7 S. Ratcoff, Phys, Rav. 21; 826 (1947).

K-8 B. D. Kern and A. H. Snell, CP-1087, 10 (1943).

Re 9 P. G. Eoonts and I. A. HaII, DDC-32. 
Lou A Langsdoss, $\mathrm{CP}-2301,6$, and $\mathrm{CP}-2638_{0}, 8$ (1944)

Low Go A. Linonberger and J. A. Hikel, LA-467 (1946)。

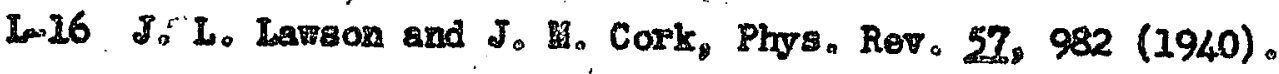

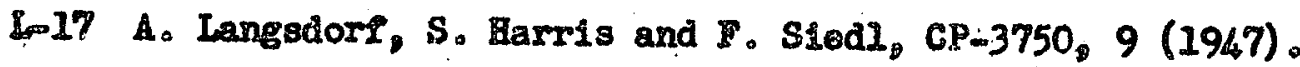

I-18 A. Lelpuneir , J. Phys。, USSR, 3, 231 (1940). See notes undor Go 13 and Gu.14.

tor-19 J. So Levinger and Stempel, CPa-1728 (1944).

Loro G. A. Linenberger, H. L. Perlman and E. Sogro, LAil6 (1943).

In-21 J. S. Levinger, CP-1067 (1942).

In22 Jo S. Levinger, CP-2267 (1944). See Lo7 for onerg notes.

I-23 H. V. Lichtenbereer and S. DancofI, CP-2638, 12 (1944).

In-24 A. Langsdorf and Arnold, CF-3403, 20 (2945). Osc1liator measurements without $c d$ absorption compered to $\sigma_{\mathrm{a}}=12.5$, and acattering to $C_{0}$ $\sigma_{0}=406$

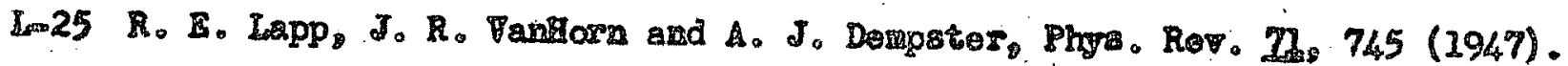

Lo-26 W. F. Libbey and Earl A. Long, Phys. Revo 25, 339 (1939).

In-27 A. Langsdorf, CP-902 (1943).

I-28 A. Iangsdorf and R. L. Purbrick, CP-3272 (1945)。 Based on $\sigma(N)=1.7$. Corrected for laok of collection of $C$ from nitride implied by new value of $C$ half-ilfe.

4-1. A. C. G. Mitcholl, E. J. Murphy and H. D. Whitaker. Phys. Rev。 50, 133 (1936). Heasured scatterling cross sections by noting increase in $\mathrm{Ag}$ detector activity when scatteror is placed next to it on the side away from incoming beam. Cd differences were not taken but corrections wero made for imperfect geometry.

16-2 J. H. Hanley, L. Jo Haworth and E. A. Lrebke, Phys。Rev. 59, 109 (1948). Measurea activation cross soctions for thernel neutrons asuring $\sigma(A)=0.2$. 


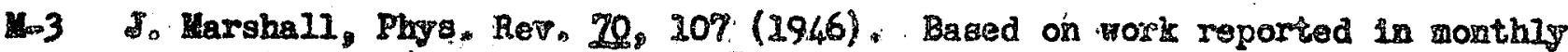
reports CP-964 and CPal088. Total erosa sections measured for In rasonance noutrons using collimated basm from CP-2 and In detretos ecrered with $1 / 8$ Ca." Background exposures wore made with $1 / 32^{m}$ In metal flltar In the bean. Saperal thicknose of samplo used and muber of folls exposed for each polat. Results belloved accurato to les or $2 \%$.

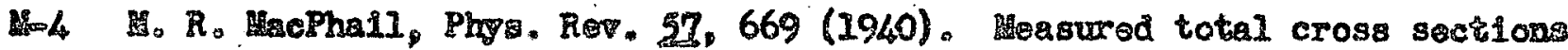
for D-D neutrons those energies lay betreen 2.38 and 2.85 met. Used $Q=3.32$. See $4-2$.

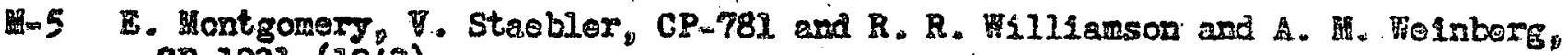
CPo1231 (1943).

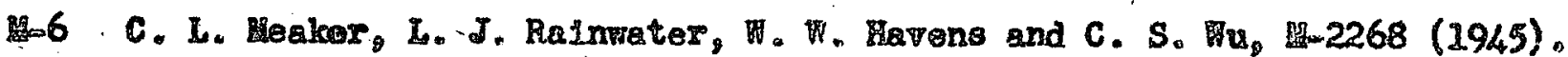

J. Marshall, CP.718.

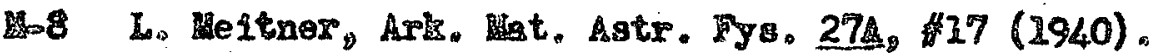

9 A. E. H1ller and Doutsch, Pho, Rer. T, 52\%, (1947).

a. Jo. Hanley, C-211 (1942).

(2) G. Monk and E. Hontgonery, CP-871 (1943).

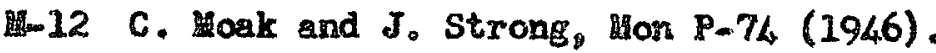

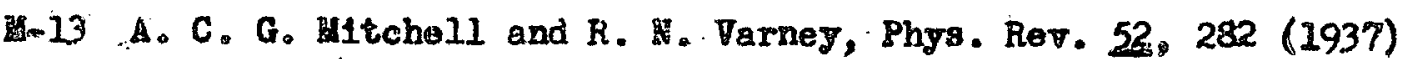

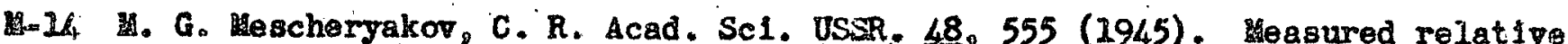
values of activation cross sections for a number of eleneats for $D-D$ neutrons of energy $\sim 2.7$ mev. Absolute values found by fitting the Au result to that found by Blalr et al (B-Il corrected for LA-520).

Ha 15 C. D. Moek and J. Strong, Hon P-56 (1946).

-16 . E. Neyerhof and G. Scharff-Goldhaber, Phys. Rev. 12, 164 (1947).

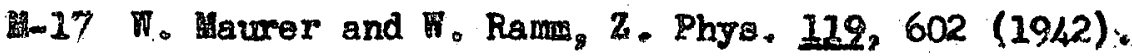

H. Haurer, Naturniss. 32, $295(19144)$.

4-20 L. Meitner, Ark.' Mat. Astr. Fys. 32A, \#6 (2945).

He 21 Ba D。 McDantel, Phys, Rev, 70, 832 (1946), See also B-19.

Mandey, L. J. Haworth and E. A. Leubire, Phys. Rev, 61, 152 (1942). 
$20 i_{4}$

Q-1 R. D. O'Neal and "Goldhaber, Phys, Rev. 59, i eross sections for $C$ neutrons using 100 on $\sigma\left(M_{n}\right)=9.4 \quad$ Volues $\sigma$ ource bsing results $12 / 9.4=1.383$.

Q-2 R. T. Overman, memorandum to L. B. Emlet, Juls 6, 1947,

P-1, L. A. Pardue, lion S-29 (1945).

P-2 H. Pomerance and J. I. Hoover, Hon P-314, 59 (19/4).

P-3 H. J. Poole and E. B. Paul, Hature 158, 482 (1946).

P-4. P. N. Powers, H, H, Goldsmith, H, G. Deyer and J. R. Dunning, Phys. Ref, 53,
947 (1938).

P-5 C. Peacock and R. G. W11kinson, Phys. Rev. 22, 251 (1947). 


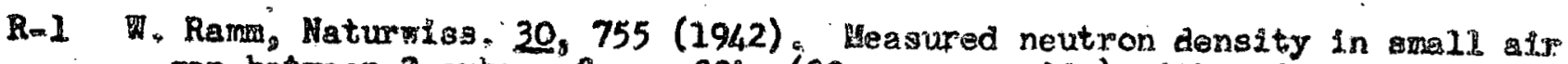
gap between 2 cubes of paraffin ( $30 \mathrm{~cm}$ on a side) with and without absorbin plates in the gap. D-Be source in plane of gap ebove paraffin. Ag indicators used and Cd differences taken. No information about purity of samples. Falues for $\sigma$ at .025 ev found by multiplying measured valuea bro factor necossary to maks Ag value equal to 57 .

R-2 F. Rasetti., Phys. Rev. 58, 896 (.1940), Hade ectivity nessurements using Ra-Be source placed inside paraffín block and small thin-walled glase counter for a detector. Ho corrections for back scattering by semple support. $C d$ differences taken. Used $\sigma(\mathrm{Mn})=9.4 \mathrm{as}$ standard. Values for $\sigma$ at .025 ev found by multiplying measured values by $13 / 9.4$.

R-3 W. Rlezler, Ann. Phys, Lpz. 41, 476 (1942). Made transwission measurenentis for 'purs' rare earth samples. In some cases analyses were provided by firms aupplying samples. Used $\sigma(0)=4$ in case of oxides. When two differont samples geve difierent results both pelues are given.

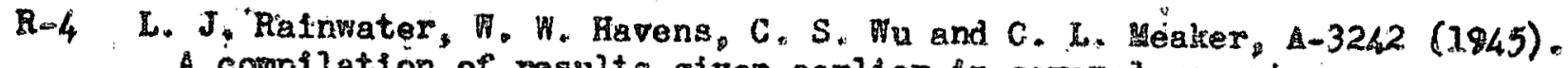
A complation of results given earlier in several reports.

Ra.5 L. J. Ralinwater and W. W. Havens, CP-2424 (1944).

R-6 Lo, J. Rainwater, W. Wavens, and C. S. Mu, H-2223 (1945).

Rm F. Rasetti, Phys. Rev, 58, 321 (1940).

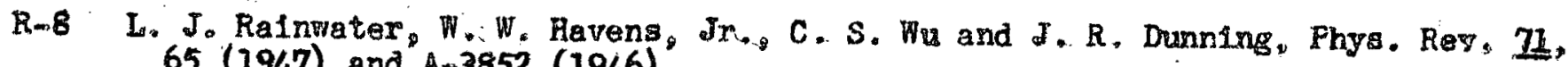
65 (1947) and A-3852 (1946).

R-9 C. H. Redman, R. Flelds, F. Friedman and B. Russe11, CF-3403, 22 (1946). Absorption measurements by sphere technique.

R-10 - W Rlezler, Phys: Z. 45, 191 (1944).

R-L L. J. Ralnwater and T. Havens, Jr., Phys. Rev. 70,136 (1946).

R-12 I. J, Rainwater and M. Havens, M-1354。

R-13 L. J. Rainmater, W. Havens and C. S. Wu, M-2259 (1945).

R. 14 I. H. Rumbaugh, R. B. Roberts and $\lambda_{0}$. Hafstad, Phys. Rev. 24, 657 (1938).

K-15 L. J. Rainwater, W. Harens and C. S, Wu, 19-2267 (1945).

R-16 W. Reirier, Ann. Phys , Lpz. 4lip 193 (1942).

R-17 L. J. Rainwater and W. Wavens; Jr., AM-2225 \#

R-18 L. J. Rafnwater and $1 . W$. Lavens, CP-2293 (1944),

R-19 8. Riother 2. Phys。 I, 367 (1946).

R-20 I. J. Rainwater and W. Havens, CP-2268 (1944). 


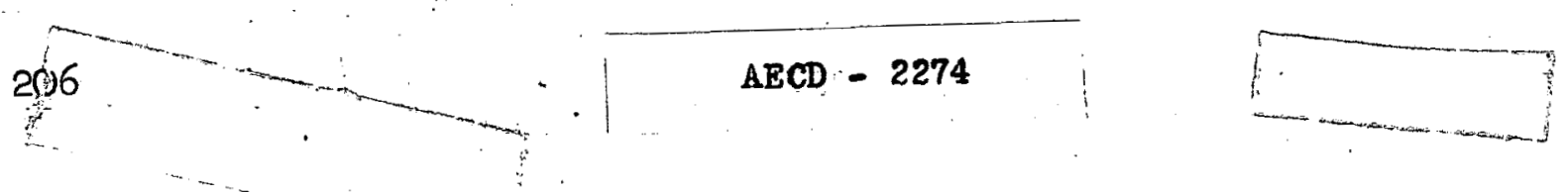

S-2 L. Seren, H. H. Friedlander and S. H. Turkel, CP-2376 (1944), Heasured sctivation cross sections. Standarized $\beta$ counting gemotry ith th and Ras otandards. Corrocted for absorption in counter wall, soll cover. and foll thickness by mesins of mass absorption coefficlents which were measured for o.ll activities. Semples wero irradiated in themel colves or in pile. All cross sections were assumed to follow $1 / v$ law. Values masured in the therral colum were corrected to values for $2200 \mathrm{~m} / \mathrm{s}$ by as of factor found nocessary to bring measured crose bections of Rh Th

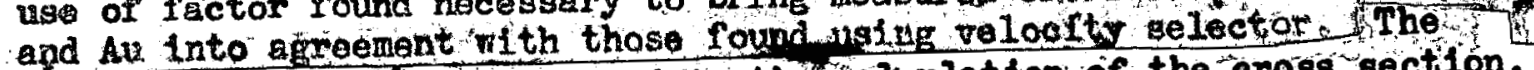
value of the half-Iife enters Into the celculation of the cross section. For long lifetines the cross section ls directly proportional to the half-Ife. Corrections were made in cases where long half-11ves the changed markediy. No mention of bàck scattering correction.

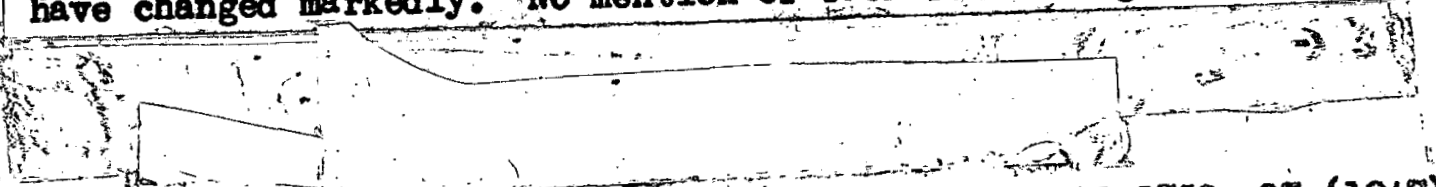

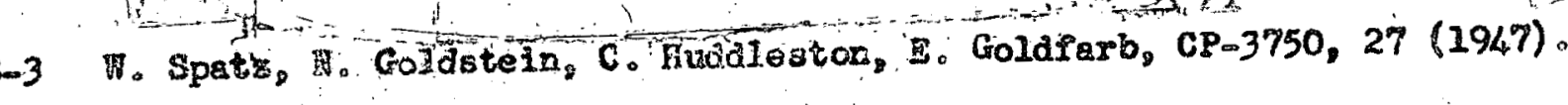

S-4 W. Stura and Jo Tabin, GP-2301 (1944)。

S-5 Sir and G. Arnold, CP-3750, 33 (2947).

-S.6 R. Spees, W. F. Colbg and S. Goudsmit, Phys. Rev. 53,326 (1938).

So7 7 slegbahn and $\mathrm{n}$. Holo, Ark. Hat. Astr. Fys.32A, 9 (1945)。

S -8 Lo Seren, F. F. Hoyer and W. Sturm, Phys. Rev. 20, 561 (1946) and CP-2376 (2944).

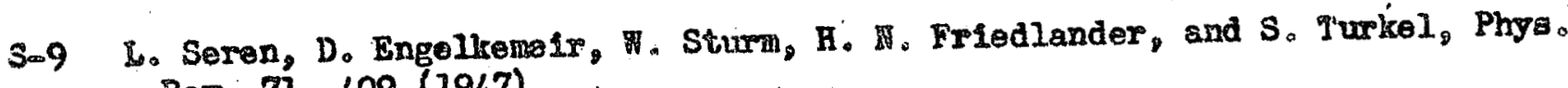
구. 71, 409 (1947).

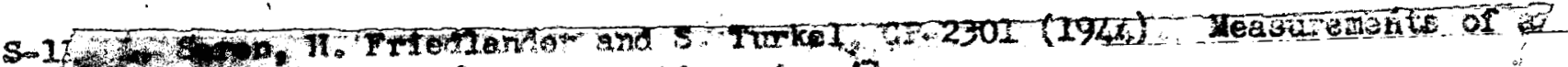

6.N.

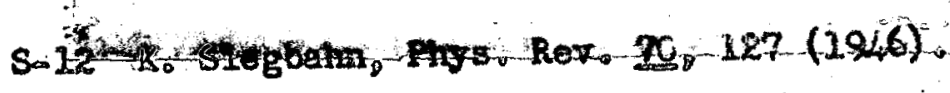

S-13 W. Sturm and S: Turkel, CP-2902 (1945)。

S-15 W. Sturm and S. Turke ${ }_{2} \mathrm{CP}-2984_{1}$ I (1945).

$S \rightarrow 26$. Sturn, Hanner and G. Arnojd, $C P-3647,20$ (1946)。

$S=17$ R. Siogbahn, Ark. Hat. Astr. Fyg, $34 \mathrm{~A}, 10$ (1946): 34B, 44 (2946).

S-19 $N$ Stiurm and $S$. Turkel, CF-3209, 20 (1945) and $C P-3195,2$ (1945). Seo elso $5-27$. 
S.20 J. A. Swartout, Mon N 229 (1946).

S-21. R. Slegbahn, Ark, Hat, Astr, Fys, 34A, (1946).

S-22 N. Sugarman, CS-2614 (1945).

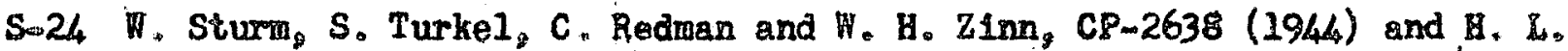

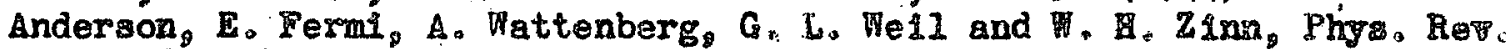
12. 26 (1947).

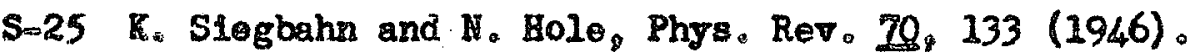

Sw26 N. Sugarman, CS-2745 (1945).

S-27 W. I. Sturm, Phys Rev。 Ig, 757 (1947). Description or Argonne cryeted spectrometer and measurements for $\mathrm{Rh}_{\dot{p}}, \mathrm{Sm}_{\mathrm{z}} \mathrm{Zu}, \mathrm{Cd}, \mathrm{Dy}, \mathrm{Ix}$ and Au, Earlier Peport in CF-3574, 4, for Sis and $\mathrm{Ev}_{2} \mathrm{CP}-2638$ for $\mathrm{Cd}, \mathrm{CF}-3209,20$, for $\mathrm{DH}$ Ir, $A u_{1}$ and $C P-3647,15$, for $E u_{\text {, }}$ Dy, Ro.

S.28 K. Siegbahn and 19.'Deutsch, Phys. Rev. In 483 (2947).

S-29 R, Sherr, Phys, Rev. 68, 240 (1945).

S.30 W. Sleator, Jr., Phgs, Revi.72, 207 (1947),

S-31. Wo Sturm and G. Arnold, $6 P-3750,41$ (1947).

S-32 R. B, Sutton, B. D, HeDaniel, E. E. Anderson, and L. S, Lavate11l, Phys, Rev, 1h, 272. (1947) and LA-92.

S-34 R, 'G. Sachs and $N_{0} G$, Goldberger, $C P, 380 I_{,} 97 \cdot(1947)$.

S-35 E, O. Salant and N. F. Ramsey, Phys. Rev. 57, 1075 (1947).

S=36 L. Seren, H. N. Friedlander, and S. Turkel, Phys. Rav. 12, 163 (1947).

S-37 W. Spatz, C. Egglex; N. Coldsteli, E. ColdParb, H, Murdoek, d. Wallace, CP-3574, $25(1946)$.

S-38 R. G. Sachs, CP-3647, 33 (1946).

S-39 J. A. Swartout, Hon 131,14 (1946). Assuming flux of $10^{12}$.

S 40 K. Slegbahn, Ark, at., Astr. Fys. 33A, \#10 (1946).

Sis 41 . A. Swartout, CP-3803, 2 (1946)。

S -42 L. C. Schulz and H. Goldhaber, Phys。 Rev。 67, 202 (1945)。

S=43 B. W. Sargent, D. V. Booker, P. E. Cavanagh, B. G. Heremard, N。 J. Wiemi

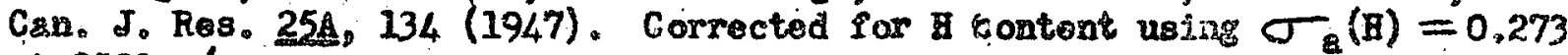
at $2500 \mathrm{w} / \mathrm{s}$ 。

S -44 H. Staub and H. Tate1, Phys, Rev. 58, 820 (1940).

S-4.5 R. Staub and I. E. Stephens, Phrs, Rev. 55, 131 (1939). 


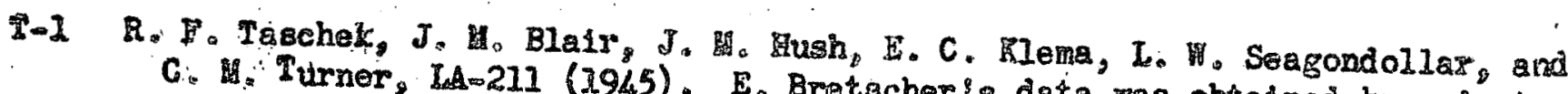
C. Turner, Jate211 (1945). E. Brotzcher's data R. F. Taschels.

T-2 A. Turkevich, Adems, Aroedman and Lo Stang, CC-2485, 5 (1944).

T.-3 A. Turkerich, E. P. Steinberg and H. Sugarman CC-2485, 3 (1944).

Yo1 H. Volz, $z_{0}$ Phys, 121, 201 (1943). Used as source two coaxlal rings filled with $\mathrm{Rn}$ and $\mathrm{Be}$ flakes which provided a rearly constant neutron flus in water between them. Found decress in sborbing sheets placed in eenters in Dy detectar activity dus to absorption of indicator and Ca differenerted for finte size of plates, $\sigma(B)=500$. Moasured and Cd difference Found $\sigma(\mathrm{Au})=72$ and $\sigma(B)=500$.

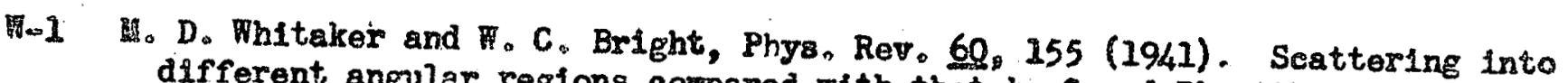
different angular regions compared with that by $C$ and $P b$ with annular Ing arrangement. Total scattering cross sectiona found by averaging cross sections maanured by the rok $\sigma(C)=4.5$ and $\sigma_{8}(P b)=9$. Total cross sections masaured by the usual transmission method. Cd differences
taken in all measurements. 


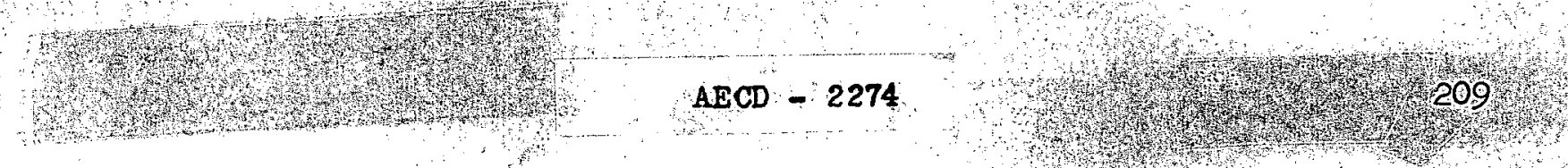

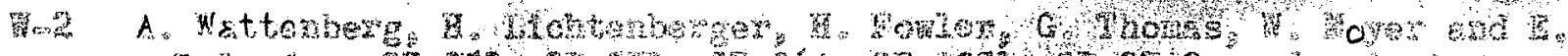

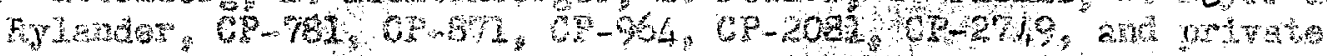

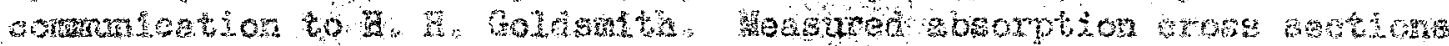

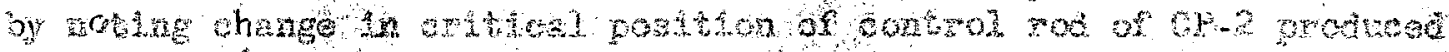

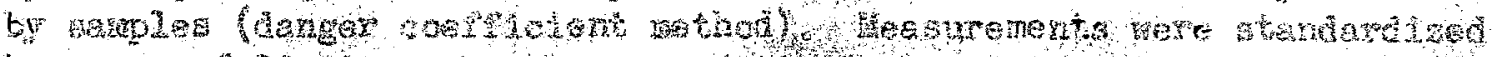

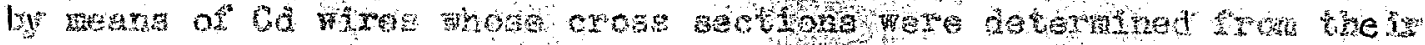

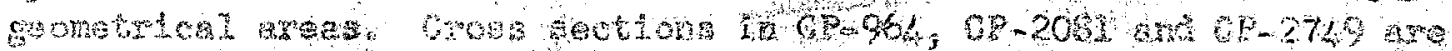

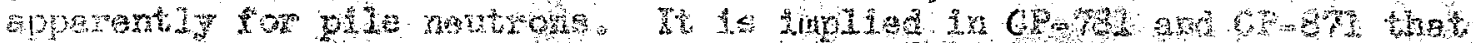

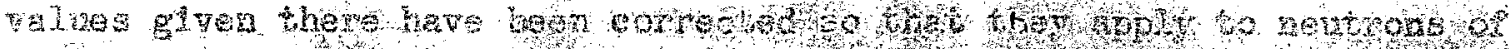

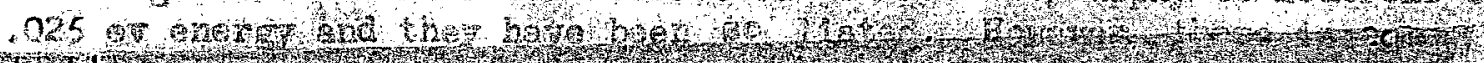
An

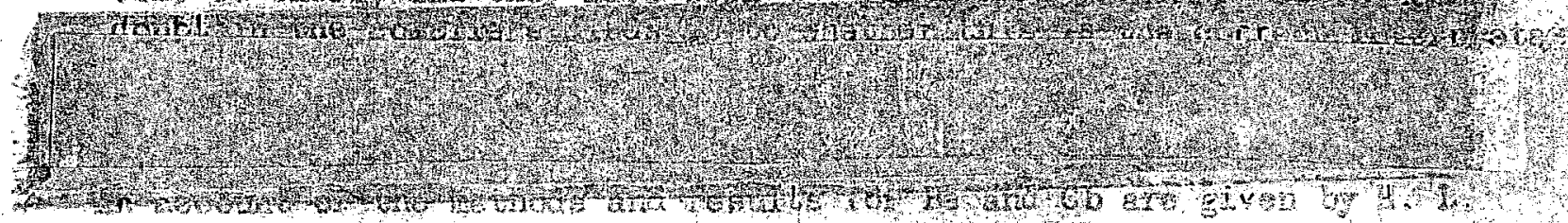

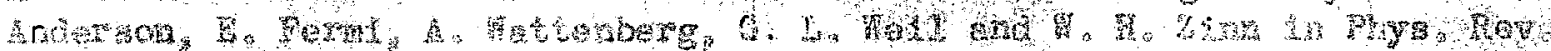

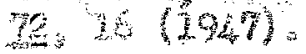

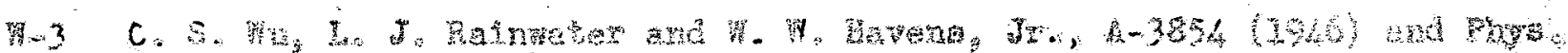

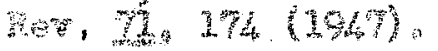

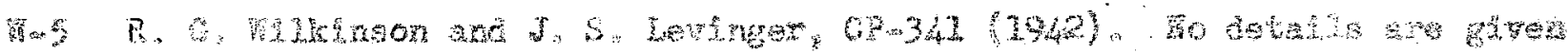

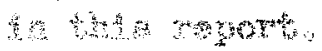

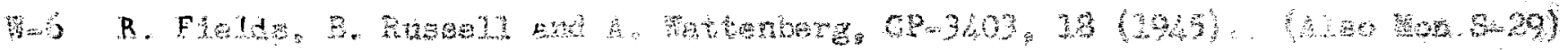

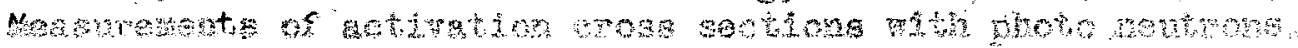

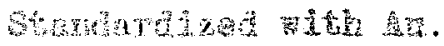

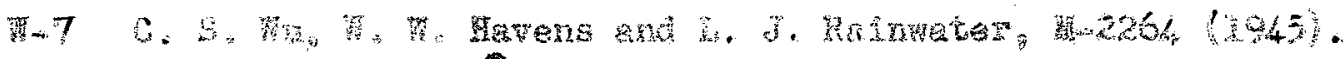

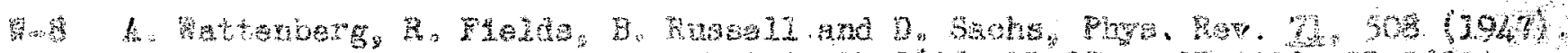

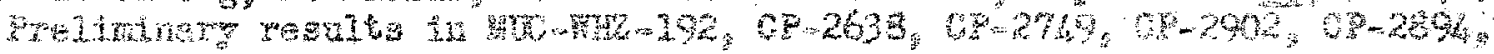

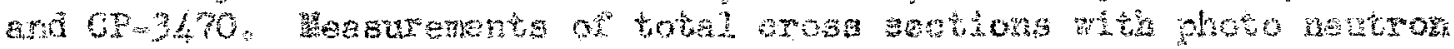

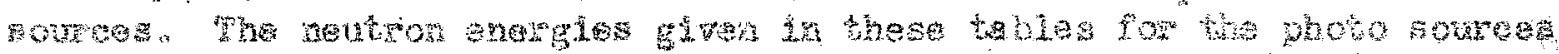

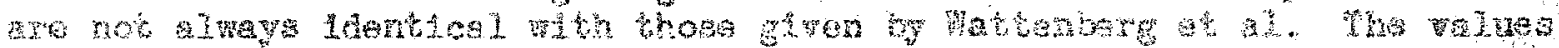

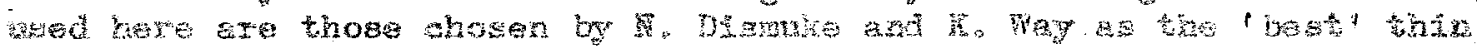

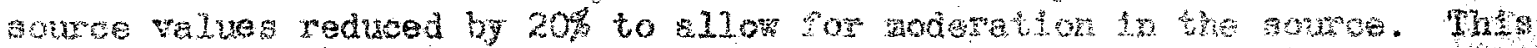

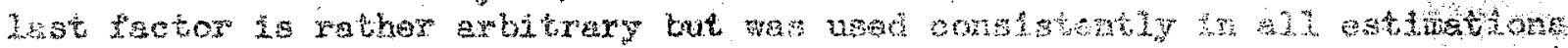

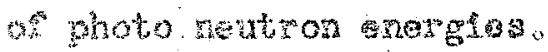

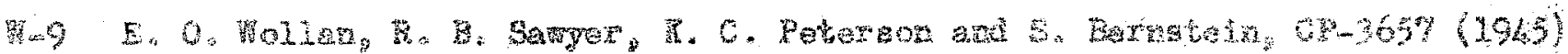

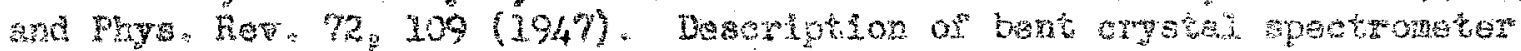

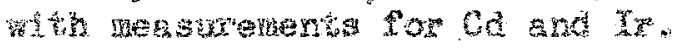

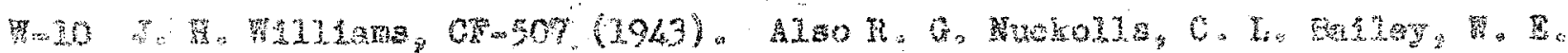

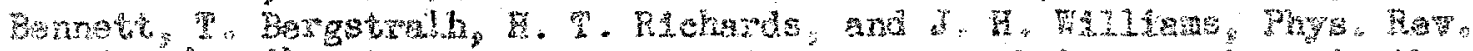

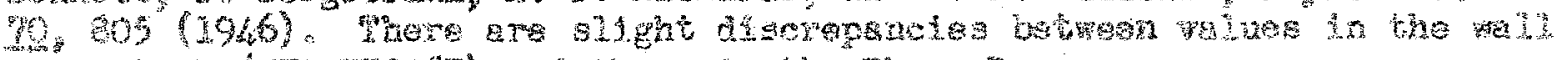

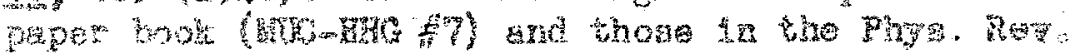

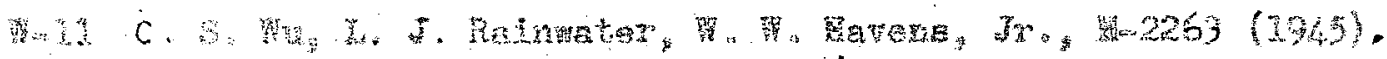


T-12 A. H. Weber, G. Armold CP-3801; 20 (1947).

Wo- 13 C.S. Tu, Io J. Rainwater and H. Havons, Jr., Ma2225 (1945).

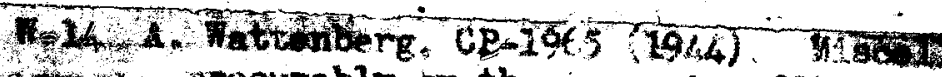

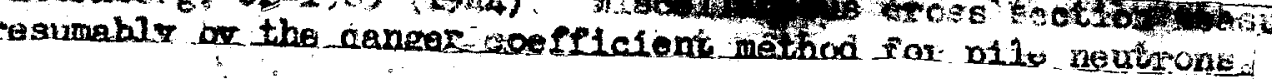

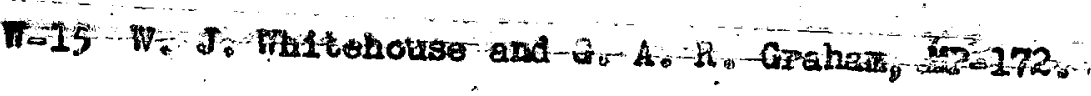

T-16. Aattonberg, CP-3364, for Maxwell diatribution of their experiment?

Z=1 M. Zlnn; S. Seoly and V. T. Cohon, Phys. Rev, 56, 260 (1939). Used lonizetion chamber Pllled with He. Anplifier set so that only recolls Which had received considerable portion of the energy mere counted. Used $Q=3.29$ Hev. Halue of $Q$ used in plotting results in wali paper
book was 3.32 . Sev. See $A-2$.

$Z \infty 2$ T. $Z \operatorname{lnn}_{0} C P-718(1943)$.

Z-3 W. H. Zlon, Phys, Reษ。 I. 752 (1947). 
It was originally hoped to include data on inelastic, transport and defferential scattering eross sections in the tables. It appearod, however, that the fob of systematizing the results would involve some correspondence and would unduly hold wo the issuing of the in part of the tables. The collection of this particules kind of data has therefore been temporarily postponed. A list of references itib a few notes is given belor.

W. D. Allen and C. Hurgt, Phys. Soc., Proc.52, 501 (1940). Determined inelastic plus absorption cross sections for a number of elements for neutrons of 3.25 mew. energy" Heasured values are not smooth function of atomse number.

E. Amaldi, D. Bocciarelli, B. N. Cacclapuoti, G. C. Trabacchi, NoCim. 30 302 (1946). Gives values of absorption plus inelastic scattering cross section for 14 mer. neutrons for $\mathrm{Al}, \mathrm{Fe}$, $\mathrm{Hg}$, and $\mathrm{Pb}$ 。

18. Aok1, Phys, Lath, Soc. Japan, Proc. 19, 367 (1937). Found relat1ve Jields of instantaneous $\gamma$ rays produced in a number of elements on bombardment with 2.4 . D-D neutrons; The $\gamma$ rays are apparently due to inelastic seattering.

E. H. Barschall, Mo E. Battat, 。 C . Bright, E. R。 Graves, T。 Jorgenseng

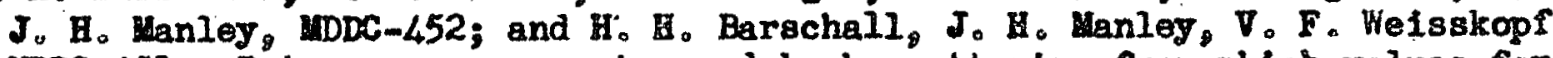
RDDC-451. Data on poor geometry and back scattering from which values for inelastie, transport, and differentlal cross soctions can be calculatsd. Elements investigated wore $\mathrm{Bo}, \mathrm{BeO}, \mathrm{B}, \mathrm{C}, \mathrm{Al}, \mathrm{F}, \mathrm{MI}, \mathrm{Co}, \mathrm{Cu}, \mathrm{Ta}, \mathrm{V}_{,} \mathrm{Au}, \mathrm{Pb}$ 。

H.H. Barschall and $R$. Ladenburg, Phgs。Rov, 61, 129 (19/2). Heasured differential cross aections for elastic seattering at acattering angles of $45^{\circ}$ and $100^{\circ}$ for $C, A 1, \mathrm{Fe}, \mathrm{Cu}_{2} \mathrm{Zn}, \mathrm{Pb}$ using $\mathrm{D}-\mathrm{D}$ neutrons of 2.5 mev e energy. 
H. F. Dunlap and R, N. Little, Phys. Rev. 60,693 (1941). Found $\sigma_{\text {in }}(\mathrm{Pb})=$ $1.31 \pm 0.53 \mathrm{~b}$ for neutrons with energies between 2.2 and 2.9 mev. Energy peak of inelastically scattered neutrons at $\sim 1.7$ mev.

D. H. Frisch and J. L. HoKibben, GF-625 (1943). Differential back scattering cross sections measured for $\mathrm{Pb}, \mathrm{Ta}_{\mathrm{y}} \mathrm{W}, \mathrm{Pt}, \mathrm{Au}$, and $\mathrm{Bi}$ for 1.6 mev neutrons. Ring geowetry. Summary of work reported in $\mathrm{CF}-304, \mathrm{CF}-348, \mathrm{CE}-392$ and CF -420 . 
S. RIkuchi, Aoki, T, Wakatuk1, PPUS Jap, 21, 410 (1939). Differential scattering cross sections for scattering angles of $23^{\circ}$ to $55^{\circ}$ for neutrons of 3.1 mev; energy for $\mathrm{C}, A 1, \mathrm{Fa}_{3} \mathrm{Cu}_{\mathrm{p}} \mathrm{Sn}, \mathrm{Fb}, \mathrm{Bi}$.

J. H. Alanley, E. H. Agnew, H. H, Barschall, W. C. Bright, J. H. Coon, E. R. Graves, $T$. Jorgensen, B. Waldman, Phys. Rev, 70, 602 (1946). Back scattering

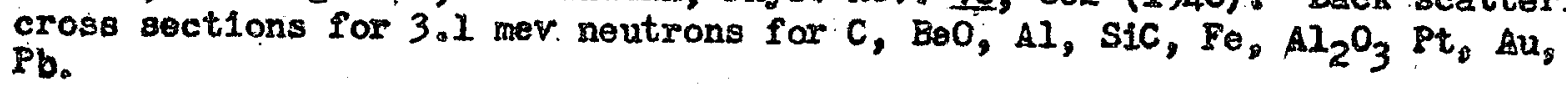

P. Olum, DDC-353, dó dsta Calculations of transport, inelastic, and differential cross sections presumably from deta of $\mathrm{lDDC}-452$ for $\mathrm{Be}, \mathrm{BeO}$,
$\mathrm{B}, \mathrm{C}, \mathrm{Al}, \mathrm{Fe}, \mathrm{W}, \mathrm{Au}, \mathrm{Pb}$.

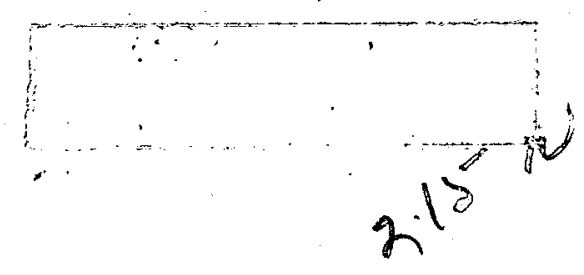




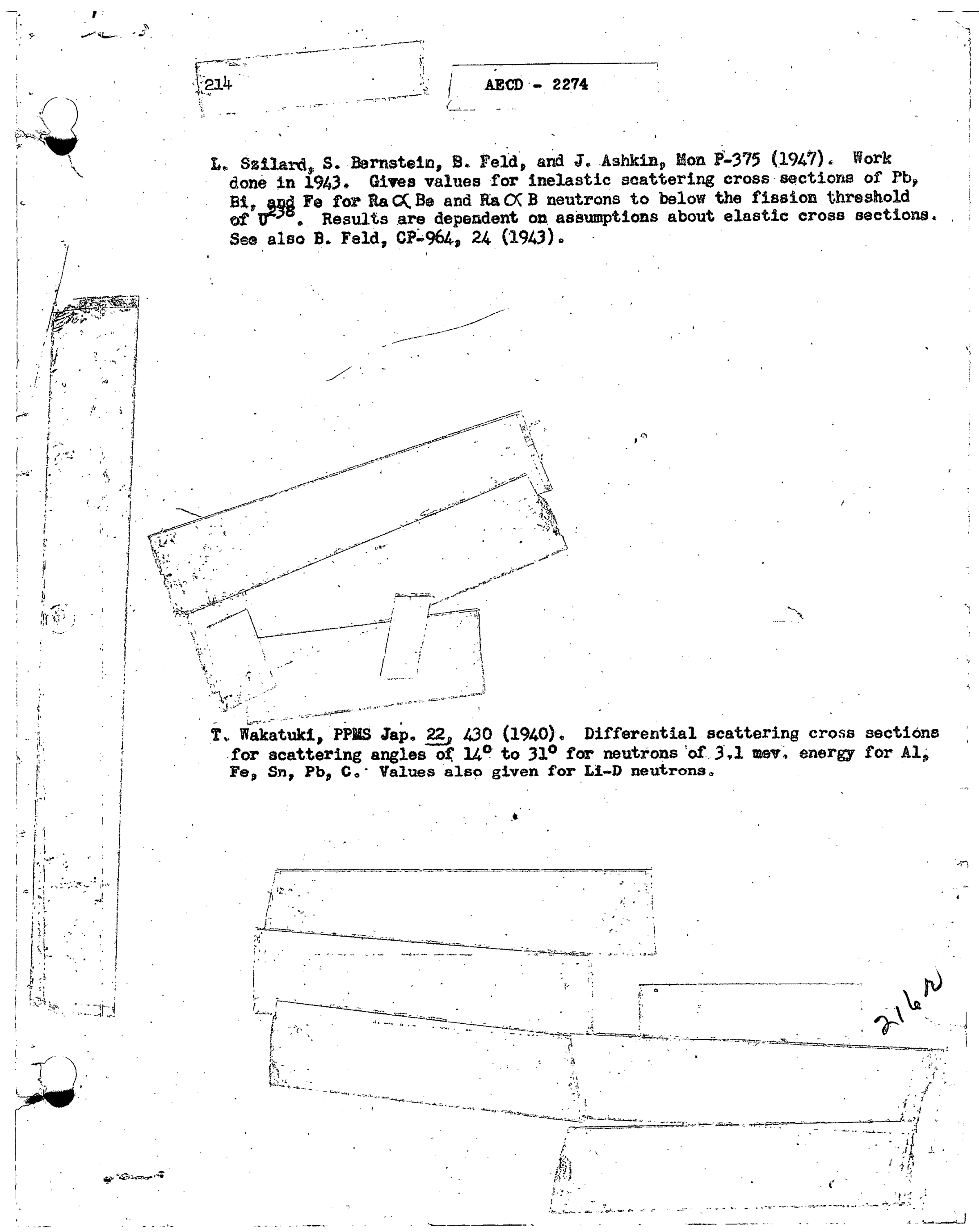




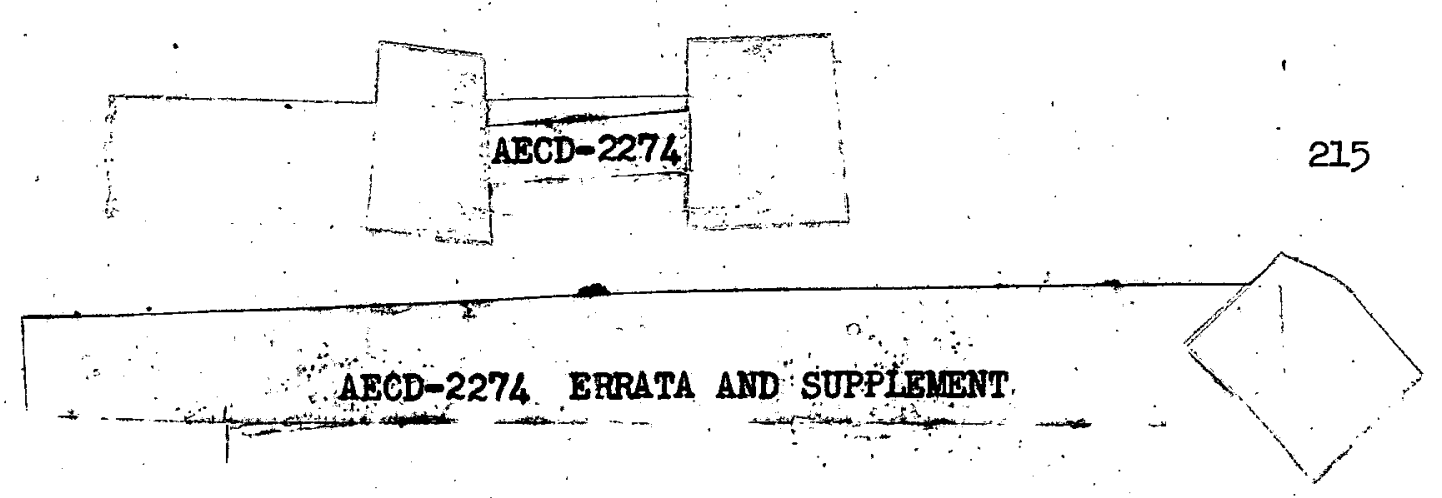

This oupplenient contains cross sections reported in the

Prratcal Reriei Vol.'TR \# 5012 (1947) and Vol. 73 \# 1-5 (1948), Argonno reportiB, ANL-4010 (1947), ANL-4076, (1947), ANL 4097 (1947) and ANL-4212 (1947), and Clinton reporte, Mion P-437 and Hon $1-432$ (1947), as well as. some that were unintentionally left out of AECD-2274.

An abbrevietions or notations not explained in this, report are explained in the earlier report.

Referonces given in AECD-2274 are not repeated in this supplement.

No attempt has been made to bring the abindances, half-lives and radiation energies up to date since these wero included primarily for purposes of orlentation. Changes in mass assigmientis have been noted ince these affect values of lootopic cross sections.

AIl. cross sections are given in barns unless otherwise stated. A half life following the oymbol for an 1sotope indlates that the crose section given is an activation cross section for that particular activity. Hew open literature or declaselfled references for data alreads Iisted in AECD-2274 are given in the reference section.

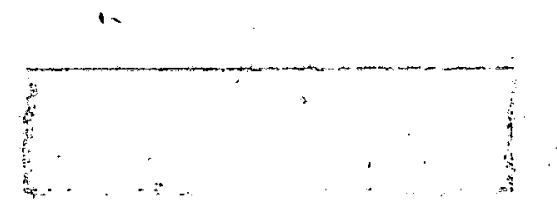




\section{ERRATA FOR MANAP}

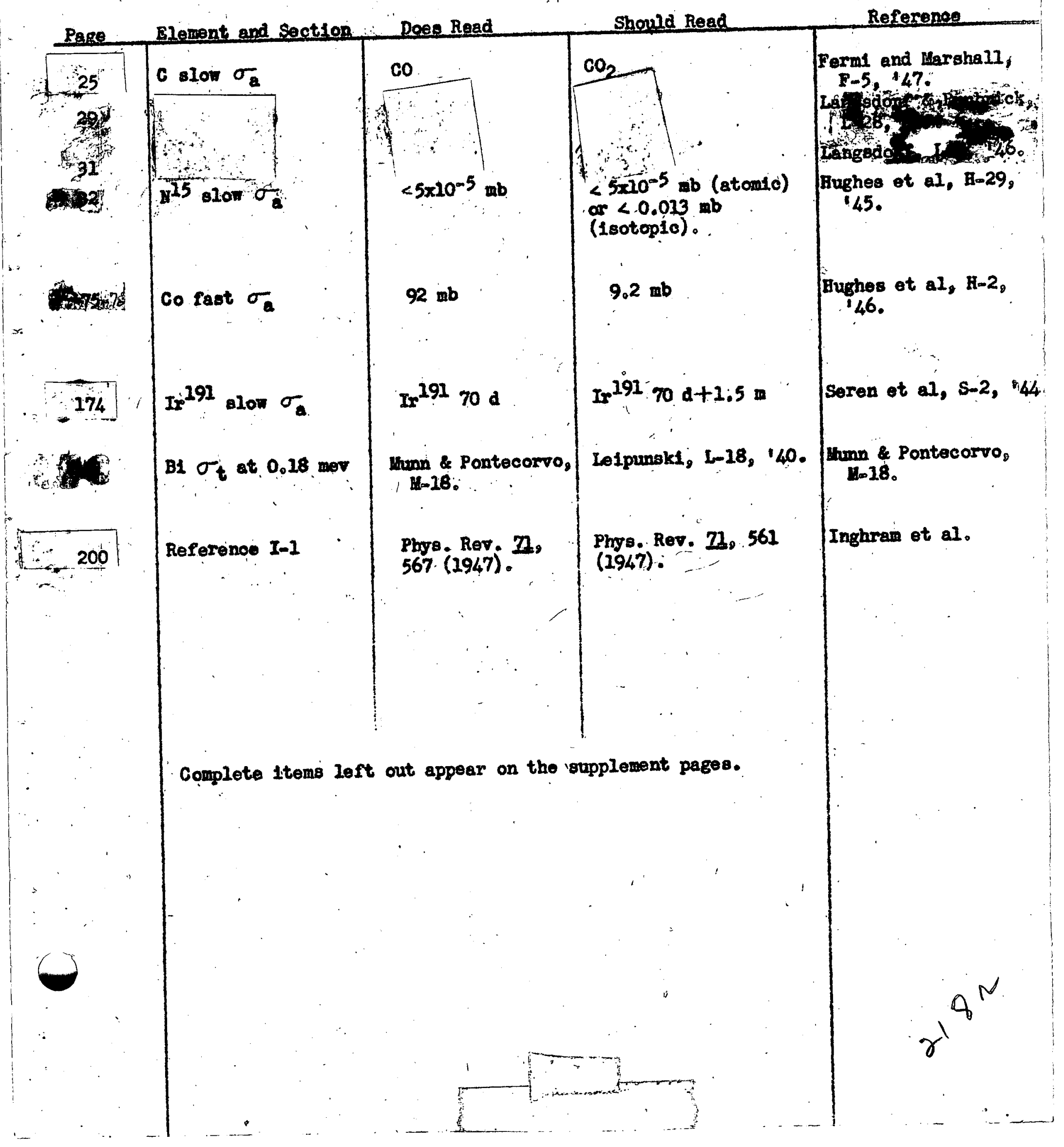




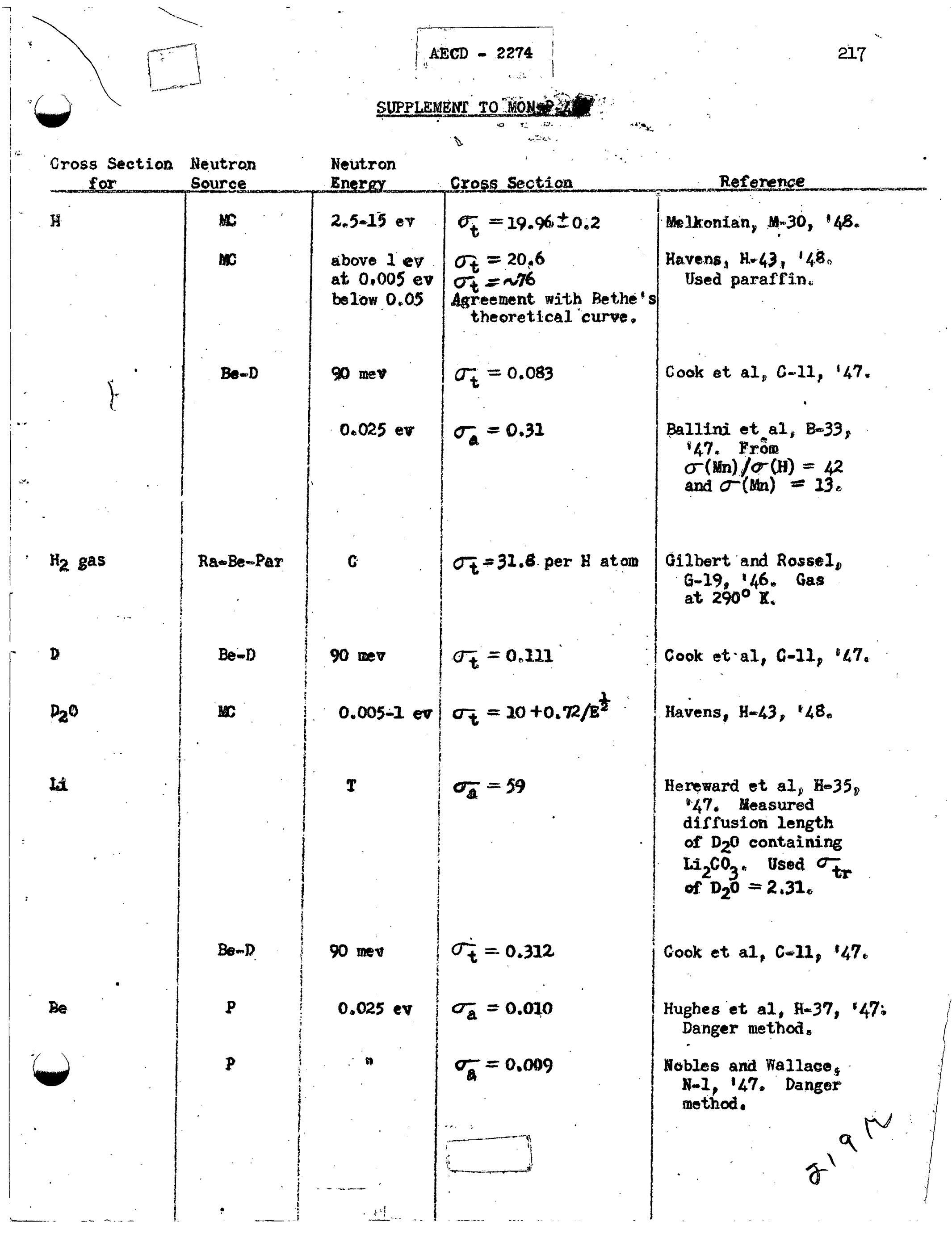




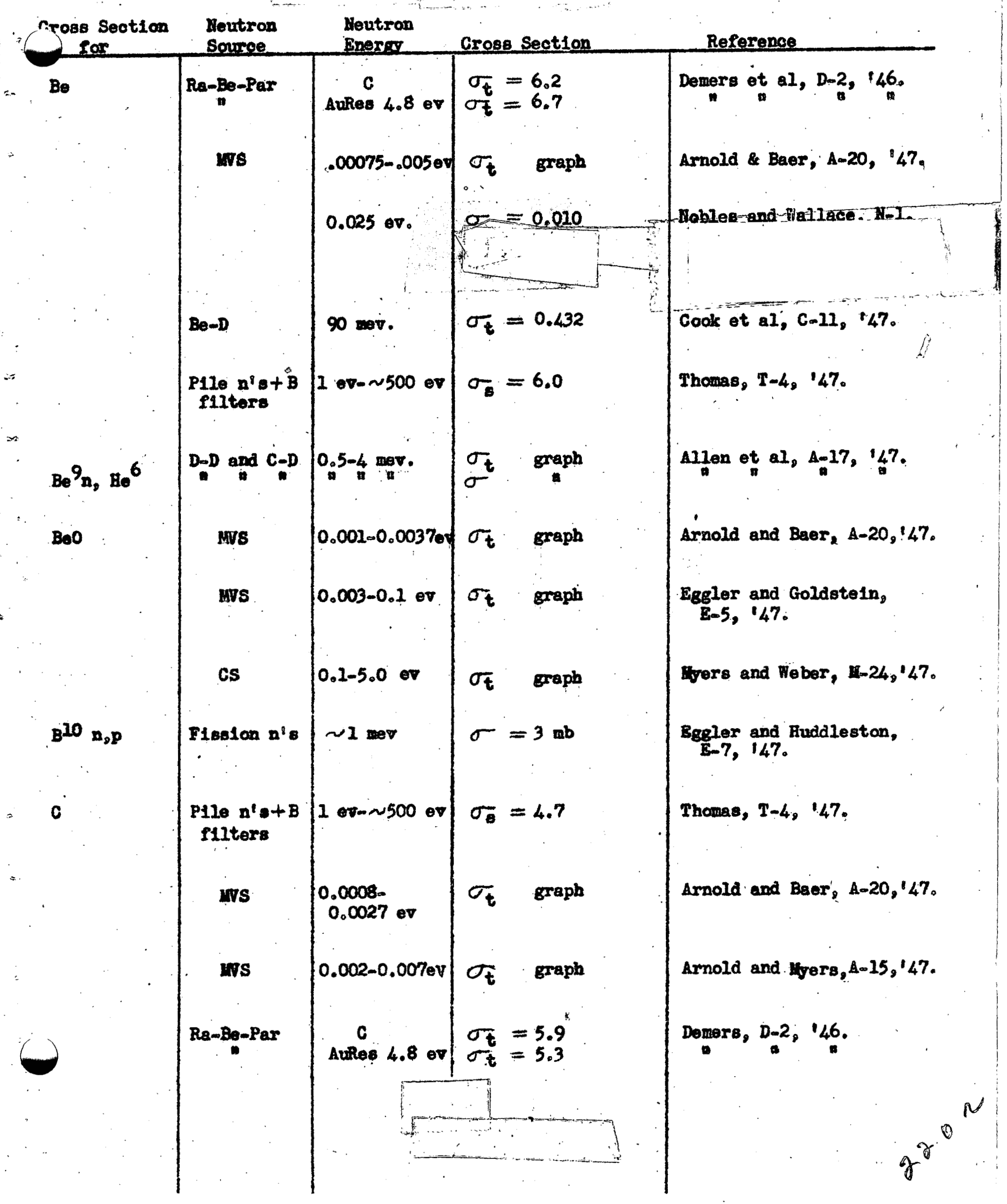




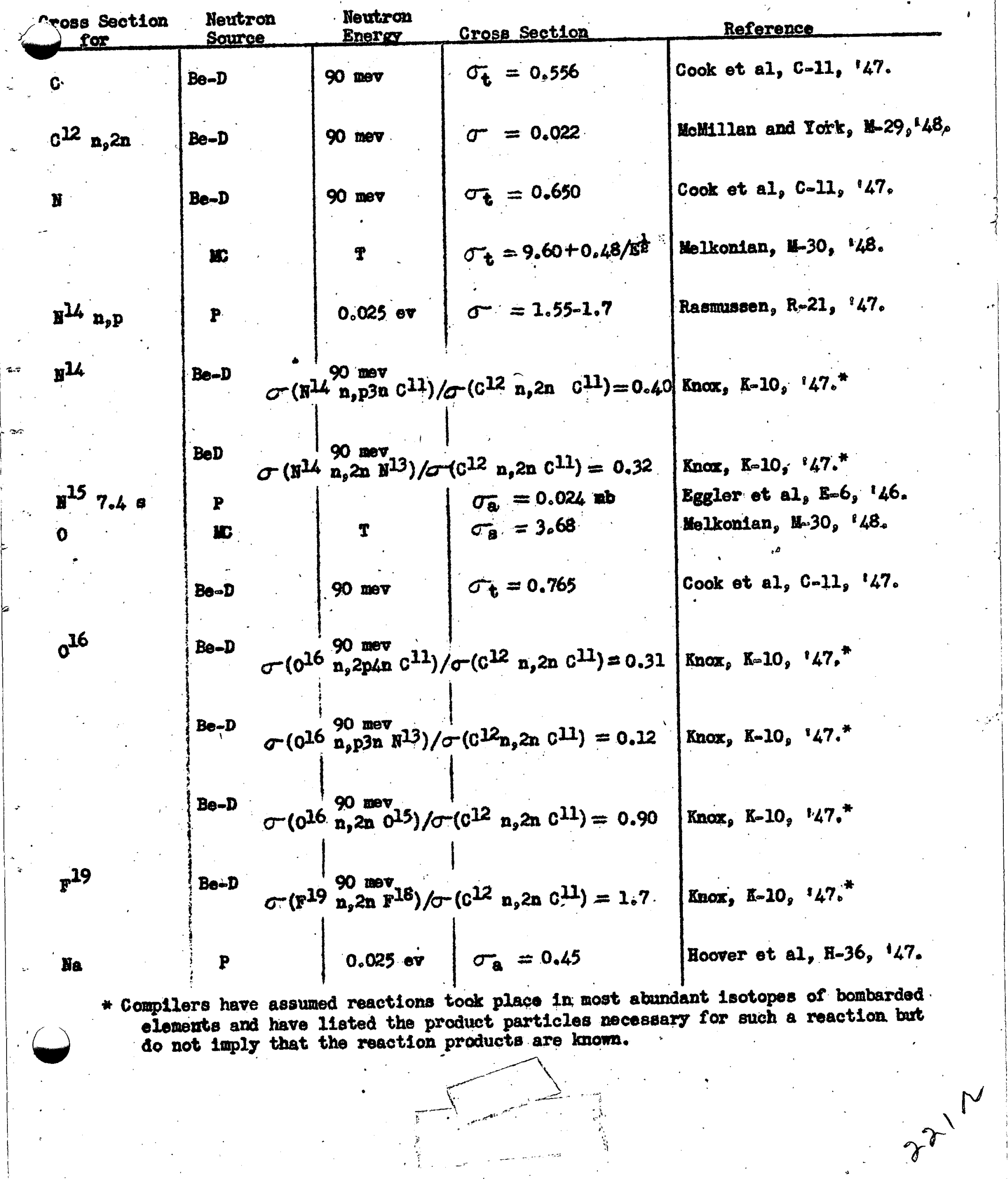




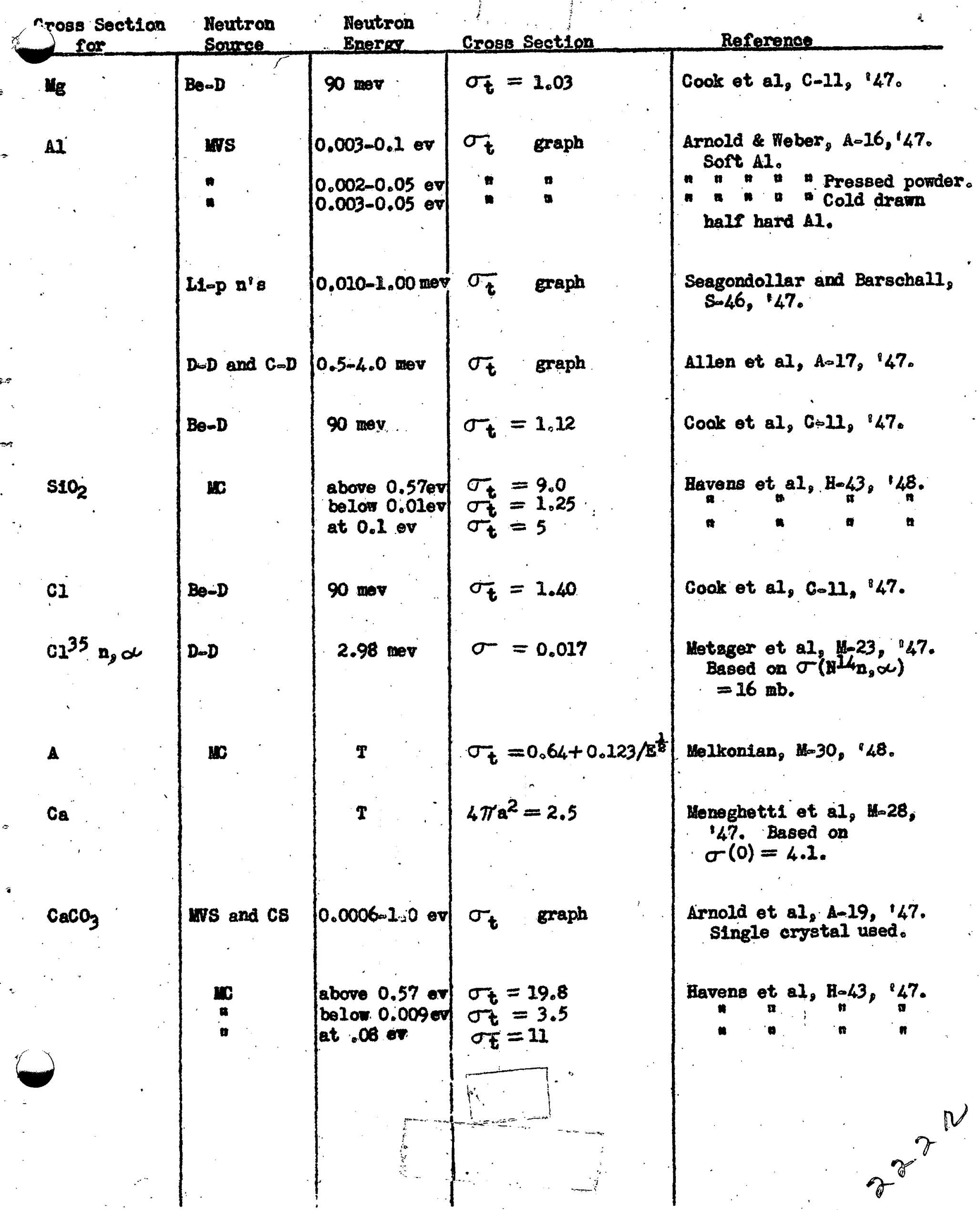




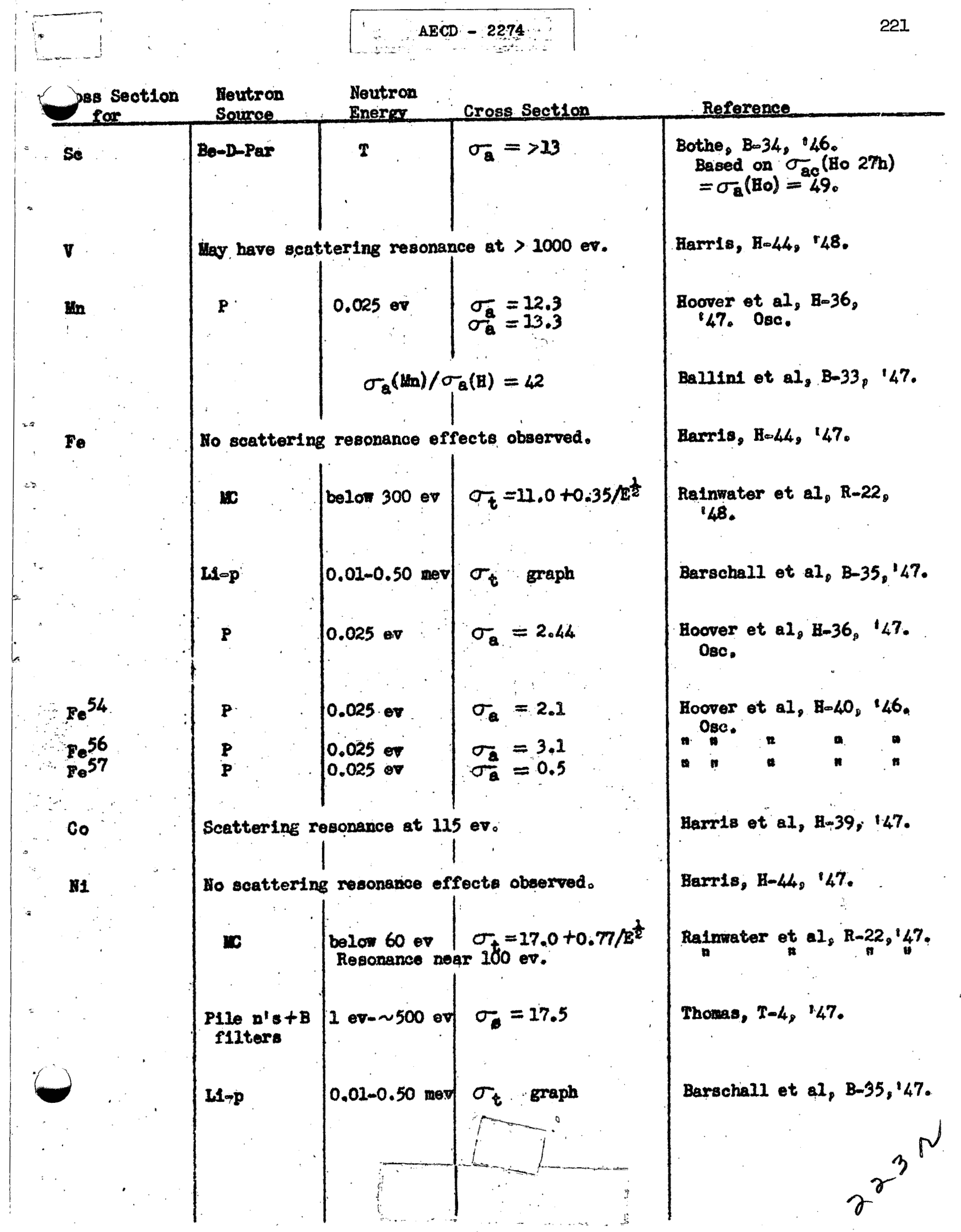




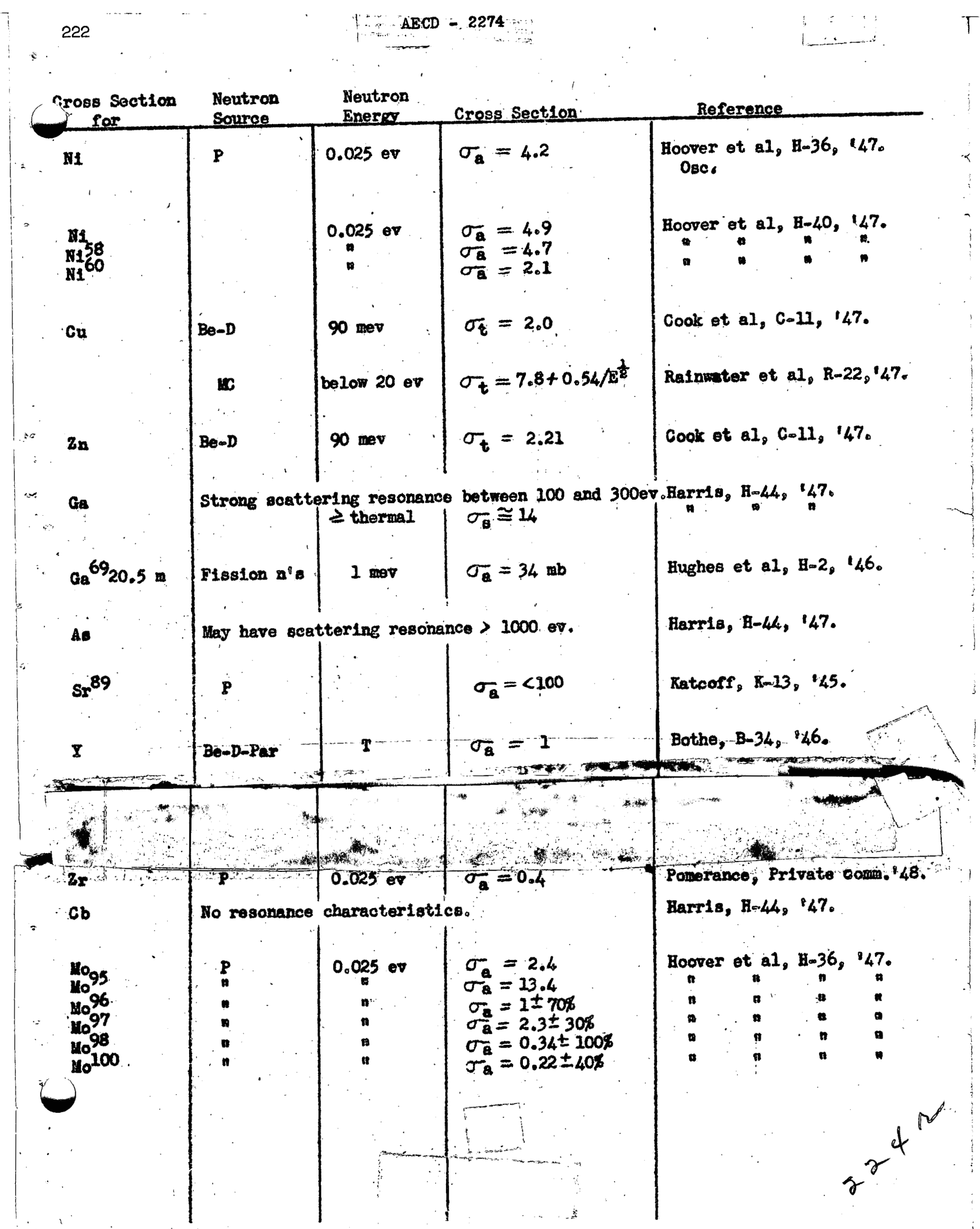




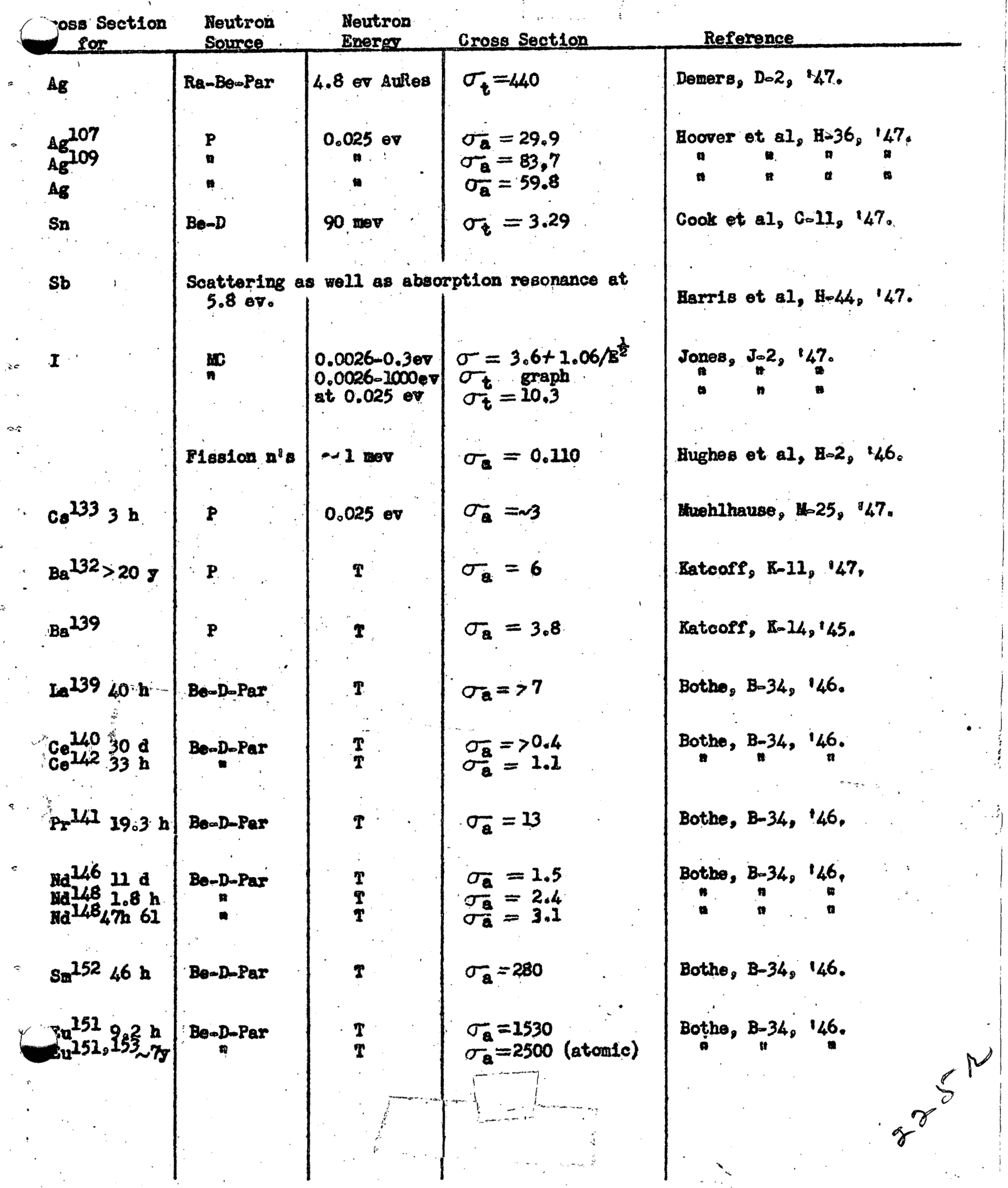




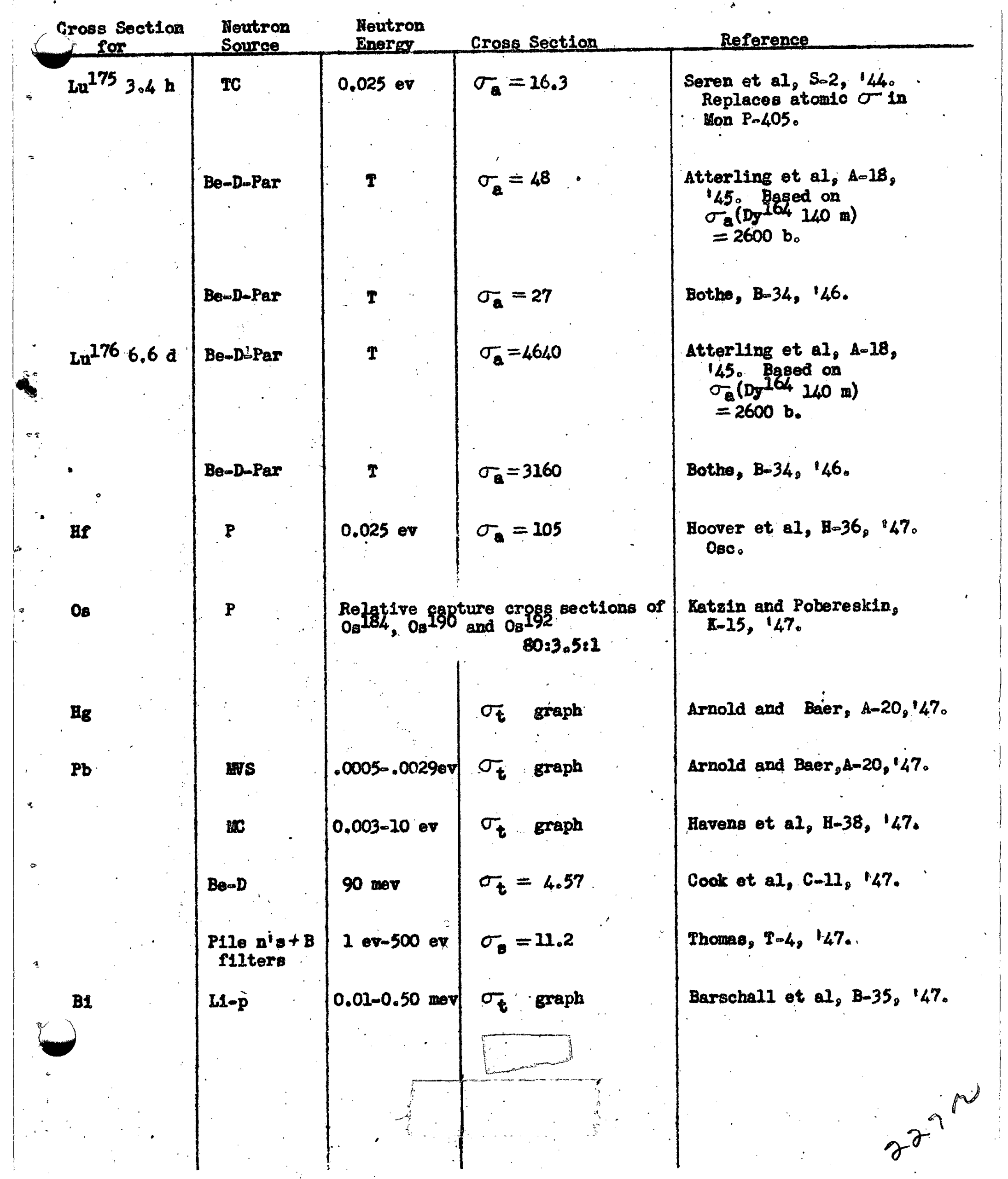


A-7 J. R. Arnold and No Sugarman, CC-3785 (1947) and J. of Chem. Phys. 15, 703 (1947).

A-9 Add: Phys. Rer. 72, 729 (1947).

A-15 G. Arnold and V. Hors, ANL 4010, 40 (1947).

A-16 G. Arnold and A. Fober, ANto-4010, 41 (2947).

A-17 R. W. Allen; W. E. Burcham and D. A. Wilkinson, Nature 159, 473 (1947).

A-18 H. Atterling, E. Bohr and Th, Sigurgeirsson, Arkiv. Mat, Astron. Pysik 324 \#2 (1945)。

A-19 G. Poi Arnold, R。 Beer and A. Ho. Weber, All-4076, 22 (1947).

A-20 G. P. Arnold and R. Baer, ANL-4076, 23 (1947). Hoasured cross sections at $300^{\circ} \mathrm{K}$ and $80^{\circ} \mathrm{K}$,

B-33 R. Bellint, A. Berthelot and C. Smeots, Comptes rendus 225, 328 (1947).

B-34 W. Bothe, 2. Naturforsch, 1, $179(1946)$, see Se, p 8, for standard.

B-35 H. H. Barschell, C. K. Boakelman and L. W. Seagondollax, MP-100 (1947).

B-38 G. E. Boyd and B. H. Ketelle, Mon N-432, 98 (1947).

C-1I L. J. Cook, E. M. Mollilian, D. C. Sewell and J. M. Peterson, BR-122 (1947)。 See also $\mathrm{DDC}-1399$.

D-10 Add: G. W. Parker, P. M. Lantz, M. G. Inghram, D. C. Hess and R. J. Hayden, Phys. Rev. $72,85(1947)$.

. E-6, C. Eggler, H. Hurdock, F。 Spats and J. Wallace, CF-349, 25 (1946).

E-7. C. Eggler and C. Huddleston, ANL-4097, 19 (1947).

Eis C. Eggler, Ant 4097,15 (1947).

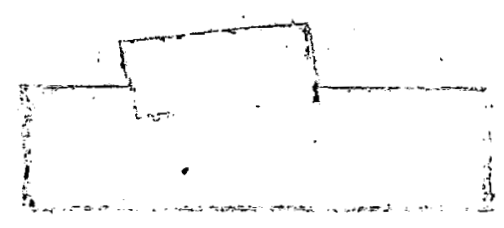


F-7 Add: Phys. Rev. 72, 408 (1947),

F-19 H. Franenfelder, P. C, Gugelot, 0. Huber, M. Hedicus, P. Preiswerk, P. Scherrer, and R. Steffen, Helv. Phys. Aota 20, 238 (1947).

G-10 Add: Phys. Rev. Di, 37R (1947).

G-18 M. Goldbaber and C. O. Nuehlhause, AML $4010_{2} 55$ (2947).

G-19 A. Gibert and Jo Rossel, Helv. Phys. Aete 19, 285 (1946).

H.2. Add: Phys. Rev. 20, 106 (19,46).

H-19 Add: E。 D。 Glema and A. O. Hanson, Phys Rev. 2, 106 (1948).

H.35 H. G. Hereward, G. C. Laurence, A. H. Hunn, H. R. Panoth and B. I. Sargent, Can. J. Res. Ae25, 26 (1947).

H-36 J. I. Hoover, H. Pomerance and W. H. Jordan, Uon P-437, 26 (1947).

H-37 D. J. Hughes, C. Eggler, and C. H. Huddleston, Phgs. Rev. I, 269. (1947).

H-38 W。 Wo Havens, JP., I. I. Rabl and Lo Jo Rainwater, Pho。 Rev。 T2, 634 (1947)。

H-39 S. P. Harris, A. S. Langsdorf, Jr., F. Q. P. Sold1, Pbos, Rev. 72, 866 (1947)。 ANL-4076, 3 (1947), ANL-4097, 8 (1947), and ANL-4020, 24 (1947).

H-40 J. I. Hoover, H. Pomerance and H. H. Jordan; Hon P-368, 44 (1946).

H-43. W. H. Havens, Jr., I. J. Rainnater and C. S. Wu, B.A.P.S. 23 \#2, 37 (1948).

H-44 S. Harris, ANL-4097, 7 (1947).

I-3 M. G. Inghram, A. E. Shat, D. C. Hess, Jr., and R. J. Hayden, Phys. Rev。 12, 515 (1947).

Jon W. B. Jones, Jr., Pluse Rev. 72, 362 (1947). 
K-10 W. J. Knox, Phys. Revo 72, 1254 (1947). Compilers assumed reactions took place in most abundant isoropes.

Ko-11 S. Katcorf, Phys。Rov。 72, 1160 (1947).

E-13 S. Ratcoff, CC-3059 (1945).

K-14 S. Ratcoff, CC-2739 (1945).

Ko15 2. I. Katzin, H. Pobereskin, ANL-4112, 26 (1947).

L-13 Add: Phys: Rev. 12, 585 (1947).

M-23 Von F. Hetzger, P. Huber and F. Alder, Helv. Phys. Acta 20, 236 (1947).

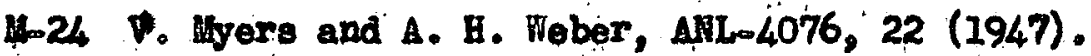

Ma25 C. O. Muehihause, All-4076, 29 (1947).

H-28 D. MeneghettI, S. So SLahu and L. Minsberg, ANL-4097 (1947).

14-29 E. H. Mollizian and H."F: York, Phys. Rev. 73, 262 (1948).

Ho-30 E. Nelkonten, B.A.P.S. 23, \#2, 37 (1948).

No1 R. Nobles and J. Mallace, ANL 4076, 10 (1947).

R-21 S. Regmassen, All-4076, 1 (1947).

R-22 I. J. Ralawater, W. W. Havens, Jx。, and C. S. Wu, B.A.P.S. 23, \#2, 36 (1948).

S-2, Add: Phys. Rov. 12, 888 (1947). For Sr see also Phos。Rev。71, 454 (1947).

S -9 Add: Phyre. Rev。 T2, 888 (1947).

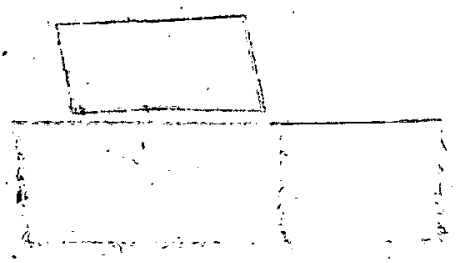


